\title{
SELECTIVE METALATION AND FUNCTIONALIZATION OF FLUORINATED NITRILES USING 2,2,6,6-TETRAMETHYLPIPERIDYL BASES
}

Thiago dos Santos (a), Henrique P. Orenha ${ }^{(a)}$, Valter E. Murie ${ }^{(a)}$, Ricardo Vessecchi ${ }^{(b)}$, Giuliano Cesar Clososki*(a,b)

a. Departamento de Ciências Biomoleculares, Faculdade de Ciências Farmacêuticas de Ribeirão Preto, Universidade de São Paulo, Avenida do Café s/n, 14040-900 Ribeirão Preto SP, Brazil.

b. Departamento de Química, Faculdade de Filosofia, Ciências e Letras de Ribeirão Preto, Universidade de São Paulo, Av. dos Bandeirantes 3900, Ribeirão Preto-SP 14090-901, Brazil.

*E-mail: gclososki@usp.br.

\section{TABLE OF CONTENTS}

I. General information

II. General procedures for the functionalization of fluorinated nitriles........................................S4

III. Reaction scope and characterization data ............................................................................................S8

IV. Examples of synthetic applications of the functionalized nitriles .............................................S39

V. Computational study ( $\mathrm{p} K \mathrm{a}$ calculations) ............................................................................................541

VI. Correlation between the calculated $\mathrm{p} K_{\mathrm{a}}$ values and metalation sites for the studied substrates

VII. NMR spectra. 


\section{GENERAL INFORMATION}

\section{Solvents and reagents}

Unless otherwise stated, all the solvents and reagents were obtained from commercial suppliers and used without prior purification. All fluorinated nitriles, salts $\left(\mathrm{ZnCl}_{2}, \mathrm{CuCl}, \mathrm{LiCl}, \mathrm{NH}_{4} \mathrm{Cl}\right.$, $\mathrm{MgSO}_{4}$ ), $i$-PrMgCl$\cdot \mathrm{LiCl}$, deuterated solvents, $\mathrm{TMPH}, \mathrm{PdCl}_{2}, \mathrm{PPh}_{3}$, and electrophiles were purchased from Merck. All the water sensitive reactions were carried out with dry solvents under anhydrous conditions and nitrogen atmosphere. The transference of the dry solvents and airsensitive reagents was carried out by means of standard syringe techniques. A saturated sodium thiosulfate solution instead of $\mathrm{NH}_{4} \mathrm{Cl}$ was used for quenching when iodine was employed as the electrophile. $\mathrm{Pd}\left(\mathrm{PPh}_{3}\right)_{4}$ was prepared according to the literature. ${ }^{1}$

TMPMgCl-LiCl: A dry round-bottom flask equipped with a stirring bar was charged with $i$ PrMgCl$\cdot \mathrm{LiCl}(1.3 \mathrm{M}$ in THF, $14 \mathrm{mmol}, 10.77 \mathrm{~mL})$ under $\mathrm{N}_{2}$. Then, fresh distilled 2,2,6,6tetramethylpiperidine (1.05 equiv., $14.7 \mathrm{mmol}, 2.48 \mathrm{~mL}$ ) was added dropwise. The flask was wrapped in aluminum foil, and the reaction was kept at room temperature for 48 hours. The resulting base was titrated with benzoic acid, and 4-(phenylazo)-diphenylamine was used as the indicator (Base concentration range: $0.87-1.1 \mathrm{M}$ ).

CuCl-2LiCl (0.5 M in THF): A dry round-bottom flask equipped with a magnetic bar was charged with $\mathrm{LiCl}(0.85 \mathrm{~g}, 20 \mathrm{mmol})$ and heated to $140{ }^{\circ} \mathrm{C}$ (oil bath) for 2 hours under vacuum. After cooling to room temperature, $\mathrm{CuCl}(0.99 \mathrm{~g}, 10 \mathrm{mmol})$ was added under nitrogen, and the temperature was adjusted to $140^{\circ} \mathrm{C}$ for $5 \mathrm{~h}$ under vacuum. After cooling, the flask was charged with dry THF $(20 \mathrm{~mL})$, covered in aluminum foil and kept under vigorous stirring to obtain a yellow homogenous solution.

$\mathrm{ZnCl}_{2}$ (1 $\mathrm{M}$ in THF): The solution was prepared by drying $\mathrm{ZnCl}_{2}(2.73 \mathrm{~g}, 20 \mathrm{mmol})$ at $150{ }^{\circ} \mathrm{C}$ (oil bath) for $5 \mathrm{~h}$ under vacuum, which was followed by the addition of dry THF (20 mL) at room temperature and vigorous stirring until all the solids were dissolved.

(TMP) ${ }_{2} \mathrm{Zn} \cdot 2 \mathrm{MgCl}_{2} \cdot 2 \mathrm{LiCl}$ : A dry round-bottom flask equipped with a stirring bar was charged with $\mathrm{ZnCl}_{2}$ (1 M, 0.53 equiv., $\left.7.10 \mathrm{mmol}, 7.10 \mathrm{~mL}\right)$, and TMPMgCl $\mathrm{LiCl}(0.87 \mathrm{M}, 13.4 \mathrm{mmol}$, $15.4 \mathrm{~mL}$ ) was added dropwise. The flask was wrapped in aluminum foil, and the reaction was kept at room temperature for $2.5 \mathrm{~h}$. The resulting solution was titrated with benzoic acid, and 4(phenylazo)-diphenylamine was used as the indicator (Base concentration $=0.29 \mathrm{M}$ ).

\footnotetext{
${ }^{1}$ Coulson, D. R.; Satek, L. C.; Grim, S. O. Tetrakis(Triphenylphosphine)Palladium(0). In Inorganic Syntheses; Cotton, F. A., Ed.; John Wiley \& Sons, Inc.: Hoboken, NJ, USA, 2007; pp 121-124.
} 
TMPH: TMPH was stirred overnight with $\mathrm{NaOH}$ beads, distilled under reduced pressure and store under nitrogen prior to use.

\section{Chromatography}

Chromatographic purification of the products was performed by flash column chromatography on silica gel (Sigma-Aldrich, particle size $0.040-0.063 \mathrm{~nm}$ ). Thin-layer chromatography (TLC) was carried out on silica plates (TLC Silica $60 \mathrm{~F}_{254}$ by Merck). Gas chromatographic investigations were performed on a Shimadzu GC-2014 chromatograph equipped with a capillary column (Restek, RTX-1, $30 \mathrm{~m} \times 0.25 \mathrm{~mm}$ ) and flame ionization detector (FID). Nitrogen gas was used as mobile phase.

\section{NMR spectra}

NMR spectra were recorded on a Bruker DRX 400 ( $\left.{ }^{1} \mathrm{H}-\mathrm{NMR}: 400 \mathrm{MHz},{ }^{13} \mathrm{C}-\mathrm{NMR}: 101 \mathrm{MHz}\right)$, Bruker DRX $500\left({ }^{1} \mathrm{H}-\mathrm{NMR}\right.$ : $500 \mathrm{MHz},{ }^{13} \mathrm{C}-\mathrm{NMR}$ : $\left.126 \mathrm{MHz}\right)$ or on a Bruker DRX $300\left({ }^{19} \mathrm{~F}-\right.$ NMR: $282 \mathrm{MHz}$ ) spectrometer. Chemical shifts are referenced to residual solvent signals (DMSO- $d_{6}: 2.50 \mathrm{ppm}$ and $39.52 \mathrm{ppm}$, and $\mathrm{CDCl}_{3}: 7.26 \mathrm{ppm}$ and $77.16 \mathrm{ppm}$ for ${ }^{1} \mathrm{H}-\mathrm{NMR}$ and ${ }^{13} \mathrm{C}-\mathrm{NMR}$, respectively), TMS (tetramethylsilane) or using the spectrometer frequency $\left({ }^{19} \mathrm{~F}\right.$ $\mathrm{NMR}, \mathrm{CDCl}_{3}$ ), and reported in parts per million (ppm). Coupling constants $(J)$ are reported in $\mathrm{Hz}$, and multiplicities of NMR signals are abbreviated as follows: bs = broad singlet, $\mathrm{s}=$ singlet, $\mathrm{d}=$ doublet, $\mathrm{dd}=$ doublet of doublets, $\mathrm{ddd}=$ doublet of doublet of doublets, $\mathrm{t}=$ triplet, $\mathrm{td}=$ triplet of doublets, $\mathrm{dt}=$ doublet of triplets, $\mathrm{qd}=$ quartet of dublets, $\mathrm{sxt}=$ sextet, $\mathrm{m}=$ multiplet and combinations thereof, app = apparent. NMR spectrometry analyses in conjunction with GC-MS and HRMS analyses were used to confirm compound identity. Additionally, we compared the obtained data with the literature for known molecules.

\section{Mass spectra}

Mass spectra (MS) was recorded on a Shimadzu GCMS-QP2010 mass spectrometer equipped with a DB-5 MS column from J\&W Scientific. Helium was used as mobile phase, and electron ionization (EI, $70 \mathrm{eV}$ ) was the ionization method. High-resolution masses (HRMS) were recorded on a Bruker Daltonics micrOTOF QII/ESI-TOF with a suitable external calibrant or on a Waters Acquity UPLC H-Class System Xevo G2-XS Q-TOF equipped with an Acquity UPLC $\mathrm{BEH} \mathrm{C}_{18}$ column $(1.7 \mu \mathrm{m}, 2.1 \times 100 \mathrm{~mm})$.

\section{Melting point}


Melting points were determined in open capillary tubes by using a BÜCHI Labortechnik M-560 melting point meter.

\section{GENERAL PROCEDURES FOR THE FUNCTIONALIZATION OF FLUORINATED NITRILES}

\section{Reaction monitoring}

Metalation of the studied nitriles was monitored by quenching reaction aliquots with an iodine solution in dry THF followed by gas chromatography analysis. To illustrate this process, the data for the metalation/optimization study of 2-fluorobenzonitrile (1b) is provided below.

Table S1. Optimization study for the magnesiation of $\mathbf{1 b}$ followed by iodination ${ }^{a}$

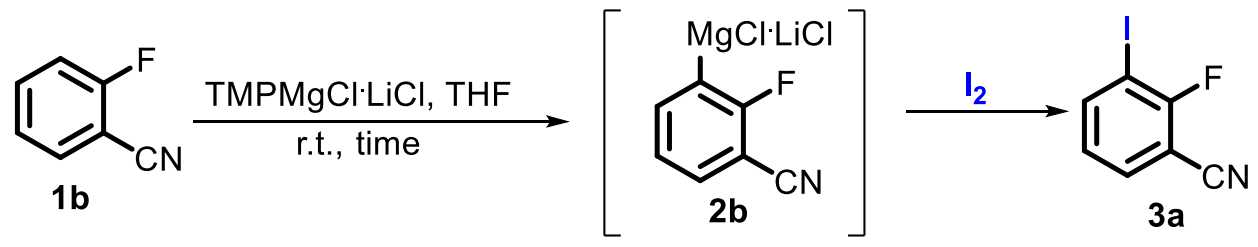

\begin{tabular}{cccccccc}
\hline Entry & Base & Solvent & Temperature & Time & $\mathbf{I}_{2}$ & $\mathbf{\% 1 b}^{b}$ & 3a:1b ratio \\
\hline $\mathbf{1}$ & 1.1 equiv. & $2 \mathrm{~mL}$ & r.t. & $1 \mathrm{~h}$ & 1.1 equiv. & 13 & $6.7: 1$ \\
$\mathbf{2}$ & 1.1 equiv. & $2 \mathrm{~mL}$ & r.t. & $2 \mathrm{~h}$ & 1.1 equiv. & 31 & $2.2: 1$ \\
$\mathbf{3}$ & 1.3 equiv. & $2 \mathrm{~mL}$ & r.t. & $1 \mathrm{~h}$ & 1.1 equiv. & 1 & $99^{c}: 1$ \\
\hline
\end{tabular}

${ }^{a} 0.5 \mathrm{mmol}$ of 2-fluorobenzonitrile (1) $)$ was considered for all the reactions. ${ }^{b}$ Remaining starting material (gas chromatography analysis). ${ }^{c}$ Isolated yield of $\mathbf{3 a}=96 \%$.

General method A (GMA): Standardized procedure for metalation of the studied fluorobenzonitriles with TMPMgCl/LiCl
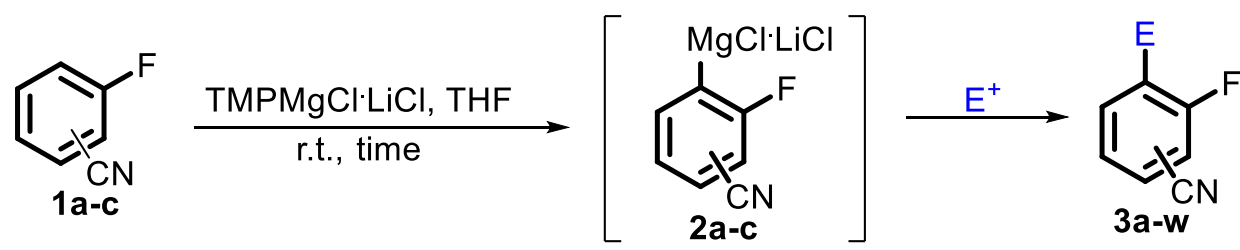

A dry round-bottom flask flushed with $\mathrm{N}_{2}$ was charged with the desired fluorobenzonitrile (0.5 mmol, 1 equiv., $0.0606 \mathrm{~g}$ ) and dry THF $(2 \mathrm{~mL})$. The base, TMPMgCl $\mathrm{LiCl}$ (1.3 equiv. for ortho and para fluoro-substituted benzonitriles and 1.2 equiv. for the meta one), was added dropwise, and the reaction was kept at room temperature for $1 \mathrm{~h}$ for the first two fluorobenzonitriles and for $0.5 \mathrm{~h}$ for the latter substrate. The generated organomagnesium species were trapped with an 
electrophile (1.1 equiv.), and the mixture was allowed to react for $12 \mathrm{~h}$. The reaction was quenched with saturated aqueous $\mathrm{NH}_{4} \mathrm{Cl}$ and extracted with ethyl acetate $(3 \times 10 \mathrm{~mL})$. The combined organic phases were dried over $\mathrm{MgSO}_{4}$, filtered and concentrated under reduced pressure. The crude product was purified by flash column chromatography (silica gel, hexanes/ethyl acetate).

\section{Sequential difunctionalization of 4-fluorobenzonitrile}

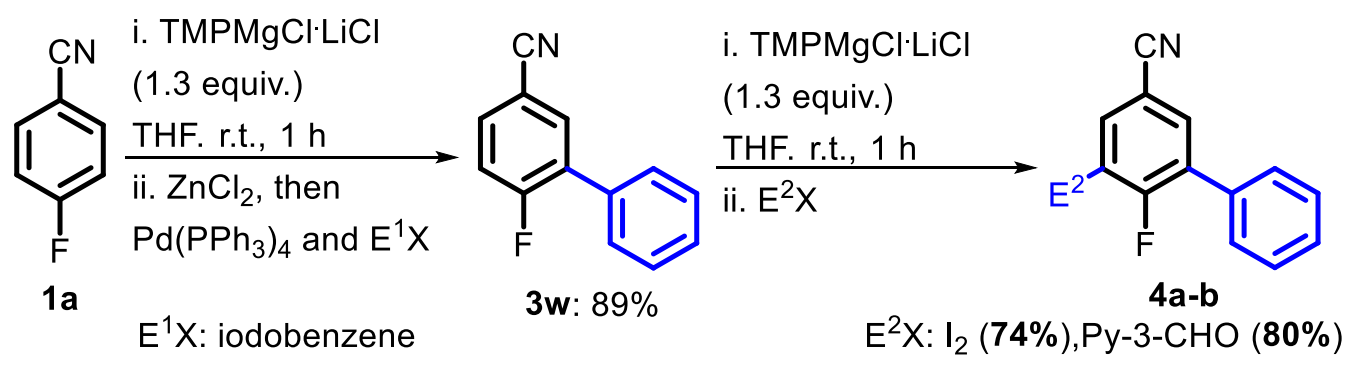

Following GMA, 3w was obtained and employed in the synthesis of 4a-b via further functionalization via the same TMP-base. The details regarding their characterization and reaction conditions are described in section IV.

\section{Gram scale synthesis of 2-fluoro-3-(hydroxy(p-tolyl)methyl)benzonitrile (3c)}

To a dry round-bottom flask flushed with $\mathrm{N}_{2}$ and charged with 2-fluorobenzonitrile (5.5 mmol, $0.6 \mathrm{~mL}, 1$ equiv.) and dry THF (22 mL), TMPMgCl $\mathrm{LiCl}(1.3$ equiv., $1.09 \mathrm{M}, 6.6 \mathrm{~mL})$ was added dropwise, and the reaction was kept at room temperature for $1 \mathrm{~h}$. The generated organomagnesium species were trapped with $p$-tolualdehyde $(1.1$ equiv., $0.78 \mathrm{~mL})$, and the mixture was allowed to react for $12 \mathrm{~h}$. The reaction was quenched with saturated aqueous $\mathrm{NH}_{4} \mathrm{Cl}$ and extracted with ethyl acetate $(3 \times 10 \mathrm{~mL})$. The combined organic phases were dried over $\mathrm{MgSO}_{4}$, filtered and concentrated under reduced pressure. Purification was accomplished by flash column chromatography (silica gel, Hexanes/AcOEt 7:3) to afford a white solid (1.21 g, $91 \%$ yield).

General method B (GMB): Standardized procedure for metalation of the studied difluorobenzonitriles with TMPMgCl$\cdot \mathrm{LiCl}$ or $(\mathrm{TMP})_{2} \mathrm{Zn} \cdot 2 \mathrm{MgCl} 2 \cdot 2 \mathrm{LiCl}$ 


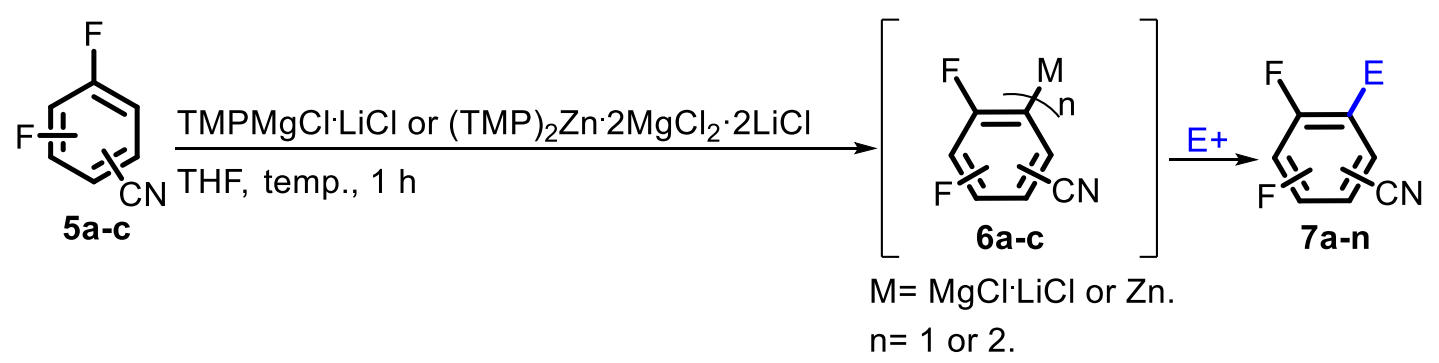

A dry round-bottom flask flushed with $\mathrm{N}_{2}$ was charged with the desired difluorobenzonitrile (0.5 mmol, 1 equiv., $0.0696 \mathrm{~g})$ and dry THF $(2 \mathrm{~mL})$, and the mixture was kept at the specified temperature. The base, TMPMgCl $\mathrm{LiCl}$ (1.3 equiv. for 2,4-difluorobenzonitrile and 3,5difluorobenzonitrile, and 1.5 equiv. for 3,4-difluorobenzonitrile) or (TMP) ${ }_{2} \mathrm{Zn}_{2} 2 \mathrm{MgCl}_{2} \cdot 2 \mathrm{LiCl}$ (0.65 equivalents for 2,4-difluorobenzonitrile and 3,5-difluorobenzonitrile, and 0.75 equivalents for 3,4-difluorobenzonitrile), was added dropwise, and the reaction was kept at the same temperature for $1 \mathrm{~h}$. The generated organomagnesium or organozinc species were trapped with an electrophile (1.1 equiv.), and the mixture was allowed to slowly reach room temperature in the case of the metalations performed under reduced temperature. The reaction was quenched with saturated aqueous $\mathrm{NH}_{4} \mathrm{Cl}$ and extracted with ethyl acetate $(3 \times 10 \mathrm{~mL})$. The combined organic phases were dried over $\mathrm{MgSO}_{4}$, filtered and concentrated under reduced pressure. The crude product was purified by flash column chromatography (silica gel, hexanes/ethyl acetate).

\section{General method C (GMC): Standardized procedure for metalation of the studied heterocyclic nitriles with $\mathrm{TMPMgCl} \cdot \mathrm{LiCl}$ or $(\mathrm{TMP})_{2} \mathrm{Zn} \cdot 2 \mathrm{MgCl} \cdot 2 \mathrm{LiCl}$}

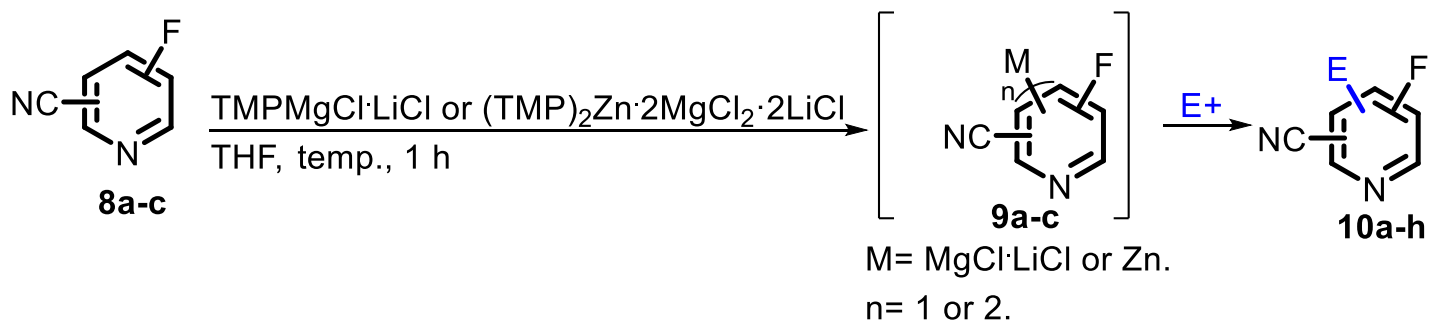

A dry round-bottom flask flushed with $\mathrm{N}_{2}$ was charged with the desired fluorinated pyridinecarbonitrile $(0.5 \mathrm{mmol}, 1$ equiv., $0.0611 \mathrm{~g})$ and dry THF $(2 \mathrm{~mL})$, and the mixture was kept at the specified temperature. The base, $\mathrm{TMPMgCl} / \mathrm{LiCl}$ (1.3 equiv. for 5fluoropicolinonitrile, and 1.5 equiv. for 2-fluoroisonicotinonitrile and 2-fluoronicotinonitrile) or (TMP) $2 \mathrm{Zn} 2 \mathrm{MgCl}_{2} \cdot 2 \mathrm{LiCl}$ ( 0.9 equiv. for 2 -fluoroisonicotinonitrile), was added dropwise, and the reaction was kept at the same temperature for $1 \mathrm{~h}$. The generated organomagnesium or organozinc species were trapped with an electrophile (1.1 equiv.), and the mixture was allowed to slowly reach room temperature in the case of metalation performed under reduced temperature. The reaction was quenched with saturated aqueous $\mathrm{NH}_{4} \mathrm{Cl}$ and extracted with ethyl 
acetate $(3 \times 10 \mathrm{~mL})$. The combined organic phases were dried over $\mathrm{MgSO}_{4}$, filtered and concentrated under reduced pressure. The crude product was purified by flash column chromatography (silica gel, hexanes/ethyl acetate).

\section{Metalation followed by Negishi cross-coupling}

After the magnesiation step (GMA), a solution of $\mathrm{ZnCl}_{2}$ (1 $\mathrm{M}$ in THF) was added dropwise at room temperature, and the reaction was kept under stirring for $0.5 \mathrm{~h}$. For the direct zincation via GMB or GMC, the organozinc species were direct considered for the next step. Then, $\operatorname{Pd}\left(\mathrm{PPh}_{3}\right)_{4}$ ( $5 \mathrm{~mol} \%)$ and the desired iodoarene (1.2 equiv.) in THF (1 mL) were transferred, and the reaction was warmed to $50{ }^{\circ} \mathrm{C}$ (oil bath) and kept under stirring for $12 \mathrm{~h}$. The reaction was quenched with saturated aqueous $\mathrm{NH}_{4} \mathrm{Cl}$ and extracted with ethyl acetate $(3 \times 10 \mathrm{~mL})$. The combined organic phases were dried over $\mathrm{MgSO}_{4}$, filtered and concentrated under reduced pressure. The crude product was purified by flash column chromatography (silica gel, hexanes/ethyl acetate).

\section{Metalation followed by transmetalation with copper salt}

After the magnesiation step (GMA), the temperature was set to $-40{ }^{\circ} \mathrm{C}$. Then, a solution of $\mathrm{CuCl} 2 \mathrm{LiCl}$ (0.5 $\mathrm{M}$ in THF, 1 equiv.) was added, and the reaction was kept at this temperature for $20 \mathrm{~min}$. Next, the temperature was increased to $-30^{\circ} \mathrm{C}$, the acyl chloride was added, and the reaction was kept under stirring for $2 \mathrm{~h}$. The reaction was quenched with saturated aqueous $\mathrm{NH}_{4} \mathrm{Cl}$ and extracted with ethyl acetate $(3 \times 10 \mathrm{~mL})$. The combined organic phases were dried over $\mathrm{MgSO}_{4}$, filtered and concentrated under reduced pressure. The crude product was purified by flash column chromatography (silica gel, hexanes/ethyl acetate).

\section{Metalation followed by palladium-catalyzed benzoylation}

To the organomagnesium and organozinc species obtained via GMA, $\mathrm{ZnCl}_{2}$ (1 M in THF) was added, and the reaction was kept at room temperature for $20 \mathrm{~min}$. Then, $\mathrm{Pd}\left(\mathrm{PPh}_{3}\right)_{4}(5 \mathrm{~mol} \%)$ and the desired acyl chloride (1 equiv.) were added. For the direct zincation via GMB, the organozinc species were directly employed with the palladium catalyst and an acyl chloride for the benzoylation. After $12 \mathrm{~h}$, the reaction was quenched with saturated aqueous $\mathrm{NH}_{4} \mathrm{Cl}$ and extracted with ethyl acetate $(3 \times 10 \mathrm{~mL})$. The combined organic phases were dried over $\mathrm{MgSO}_{4}$, 
filtered and concentrated under reduced pressure. The crude product was purified by flash column chromatography (silica gel, hexanes/ethyl acetate).

\section{REACTION SCOPE AND CHARACTERIZATION DATA}

\section{2-Fluoro-3-iodobenzonitrile (3a)}

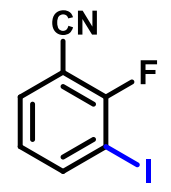

2-fluoro-3-iodobenzonitrila was obtained from 1b via GMA using TMPMgCl-LiCl (1 M, 1.3 equiv., $0.65 \mathrm{~mL}$ ) and iodine (1.1 equiv., $0.55 \mathrm{mmol}, 139.6 \mathrm{mg})$. Purification via column chromatography (Hexanes/AcOEt 9.5:0.5) afforded the product as a yellow solid (0.48 mmol, $119.0 \mathrm{mg}, 96 \%)$. CAS: 211943-27-0.

Mp: $44-47^{\circ} \mathrm{C}$.

${ }^{1}$ H-NMR (400 MHz, CDCl3): $\boldsymbol{\delta}[\mathbf{p p m}]=8.01(\mathrm{ddd}, J=7.8,6.0,1.6 \mathrm{~Hz}, 1 \mathrm{H}), 7.61$ (ddd, $J=7.6$, 5.8, 1.6 Hz, 1H), 7.05-7.01 (m, 1H).

${ }^{13} \mathbf{C}\left\{{ }^{1} \mathbf{H}\right\}$ NMR (101 MHz, CDCl$): \delta[p p m]=162.3\left(\mathrm{~d}, J_{\mathrm{C}-\mathrm{F}}=256.7 \mathrm{~Hz}\right), 144.5,133.6,126.3$ $\left(\mathrm{d}, J_{\mathrm{C}-\mathrm{F}}=4.3 \mathrm{~Hz}\right) 113.1,102.1\left(\mathrm{~d}, J_{\mathrm{C}-\mathrm{F}}=18.3 \mathrm{~Hz}\right), 82.1\left(\mathrm{~d}, J_{\mathrm{C}-\mathrm{F}}=24.0 \mathrm{~Hz}\right)$.

${ }^{19}$ F NMR (282 MHz, CDCl3): $\delta$ [ppm] $=-85.98(\mathrm{t}, J=5.9 \mathrm{~Hz}, 1 \mathrm{~F})$.

HRMS (ESI-TOF) m/z: [M+Na] ${ }^{+}$Calcd for $\mathrm{C}_{7} \mathrm{H}_{3}$ FINNa 269.9186; Found 269.9184.

The reported characterization data is in accordance with the literature. ${ }^{2}$

\section{3-Fluoro-2-iodobenzonitrile (3b)}

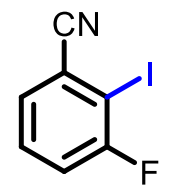

3-fluoro-2-iodobenzonitrile was obtained from 1a via GMA using TMPMgCl LiCl (1 M, 1.2 equiv., $0.60 \mathrm{~mL})$ and iodine (1.1 equiv., $0.55 \mathrm{mmol}, 139.6 \mathrm{mg})$. Purification via column

\footnotetext{
2 Eastwood, P. R.; Gonzalez, R. J.; Giulio, M. V. New substituted indolin-2-one derivatives and their use as P39 mitogenactivated kinase inhibitors. U.S. Patent WO 2009/132774 A1.
} 
chromatography (Hexanes/AcOEt 9.5:0.5) afforded the product as a yellow solid (0.34 mmol, 84 mg, 68\%). CAS: 916792-62-6.

Mp: $86-88{ }^{\circ} \mathrm{C}$.

${ }^{1} \mathbf{H}-N M R(400$ MHz, CDCl 3$): \delta[\mathbf{p p m}]=7.49-7.43(\mathrm{~m}, 2 \mathrm{H}), 7.31-7.25(\mathrm{~m}, 1 \mathrm{H})$.

${ }^{13} \mathbf{C}\left\{{ }^{1} \mathbf{H}\right\}$ NMR (101 MHz, CDCl3): $\delta[\mathbf{p p m}]=162.6\left(\mathrm{~d}, J_{\mathrm{C}-\mathrm{F}}=248.6 \mathrm{~Hz}\right), 130.8\left(\mathrm{~d}, J_{\mathrm{C}-\mathrm{F}}=8.1\right.$ $\mathrm{Hz}), 130.4\left(\mathrm{~d}, J_{\mathrm{C}-\mathrm{F}}=3.7 \mathrm{~Hz}\right), 122.7,119.9\left(\mathrm{~d}, J_{\mathrm{C}-\mathrm{F}}=24.3 \mathrm{~Hz}\right), 118.5\left(\mathrm{~d}, J_{\mathrm{C}-\mathrm{F}}=3.5 \mathrm{~Hz}\right), 87.1(\mathrm{~d}$, $\left.J_{\mathrm{C}-\mathrm{F}}=29.1 \mathrm{~Hz}\right)$.

${ }^{19}$ F NMR (282 MHz, CDCl 3$)$ : $\delta[p p m]=-87.24--87.33(\mathrm{~m}, 1 \mathrm{~F})$.

HRMS (ESI-TOF) m/z: [M+H] ${ }^{+}$Calcd for $\mathrm{C}_{7} \mathrm{H}_{4} \mathrm{FIN} 247.9367$; Found 247.9366.

\section{4-Fluoro-3-iodobenzonitrile (3c)}

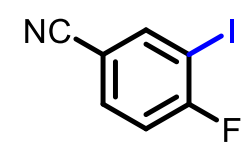

4-fluoro-3-iodobenzonitrile was obtained from 1c via GMA using TMPMgCl LiCl (1 M, 1.3 equiv., $0.65 \mathrm{~mL})$ and iodine (1.1 equiv., $0.55 \mathrm{mmol}, 139.6 \mathrm{mg})$. Purification via column chromatography (Hexanes/AcOEt 9.5:0.5) afforded the product as a yellow solid (0.41 mmol, $101.3 \mathrm{mg}, 82 \%)$. CAS: 159719-57-0.

Mp: $55-58^{\circ} \mathrm{C}$.

${ }^{1}$ H-NMR (400 MHz, CDCl3): $\boldsymbol{\delta}[\mathbf{p p m}]=8.07(\mathrm{dd}, J=5.7,2.0 \mathrm{~Hz}, 1 \mathrm{H}), 7.65$ (ddd, $J=8.5,4.6$, $2.0 \mathrm{~Hz}, 1 \mathrm{H}), 7.17(\mathrm{dd}, J=8.5,7.4 \mathrm{~Hz}, 1 \mathrm{H})$.

${ }^{13} \mathbf{C}\left\{{ }^{1} \mathbf{H}\right\}$ NMR $\left(101 \mathrm{MHz}, \mathbf{C D C l}_{3}\right): \delta[\mathbf{p p m}]=164.6\left(\mathrm{~d}, J_{\mathrm{C}-\mathrm{F}}=255.0 \mathrm{~Hz}\right), 143.5,134.5\left(\mathrm{~d}, J_{\mathrm{C}-\mathrm{F}}=\right.$ $8.9 \mathrm{~Hz}), 116.9,116.6,110.5\left(\mathrm{~d}, J_{\mathrm{C}-\mathrm{F}}=4.1 \mathrm{~Hz}\right), 82.3\left(\mathrm{~d}, J_{\mathrm{C}-\mathrm{F}}=27.3 \mathrm{~Hz}\right)$.

${ }^{19}$ F NMR (282 MHz, CDCl3): $\delta[\mathbf{p p m}]=-83.16--83.23(\mathrm{~m}, 1 \mathrm{~F})$.

GC-MS (EI, 70 eV) m/z: 246.95 (100\%), 120.05 (58\%), 100.05 (21\%), 93.00 (10\%), 247.95 $(8 \%)$.

The reported characterization data is in accordance with the literature. ${ }^{3}$

\footnotetext{
${ }^{3}$ Chambers, R. D.; Skinner, C. J.; Atherton, M. J.; Moilliet, J. S. Elemental Fluorine. Part 4. Use of Elemental Fluorine for the Halogenation of Aromatics. J. Chem. Soc. Perkin 1 1996, No. 14, 1659.
} 
<smiles>N#Cc1cccc(C(O)c2ccc(C(F)(F)F)cc2)c1F</smiles>

2-fluoro-3-(hydroxy(4-(trifluoromethyl)phenyl)methyl)benzonitrile was obtained from $\mathbf{1 b}$ via GMA using TMPMgCl$\cdot \mathrm{LiCl}(1 \mathrm{M}, 1.3$ equiv., $0.65 \mathrm{~mL})$ and 4-(trifluoromethyl)benzaldehyde (1.1 equiv., $0.55 \mathrm{mmol}, 75 \mu \mathrm{L}$ ). Purification via column chromatography (Hexanes/AcOEt 8:2) afforded the product as a yellow solid $(0.27 \mathrm{mmol}, 80 \mathrm{mg}, 54 \%)$.

Mp: $112-114^{\circ} \mathrm{C}$.

${ }^{1}$ H-NMR (400 MHz, CDCl3): $\boldsymbol{\delta}$ [ppm] = 7.86 - $7.82(\mathrm{~m}, 1 \mathrm{H}), 7.62(\mathrm{~d}, J=8.2 \mathrm{~Hz}, 2 \mathrm{H}), 7.57$ $7.52(\mathrm{~m}, 3 \mathrm{H}), 7.32-7.28(\mathrm{~m}, 1 \mathrm{H}), 6.22(\mathrm{~s}, 1 \mathrm{H}), 2.78(\mathrm{bs}, 1 \mathrm{H})$.

${ }^{13} \mathbf{C}\left\{{ }^{1} \mathbf{H}\right\}$ NMR (101 MHz, CDCl $): \delta[\mathbf{p p m}]=160.1\left(\mathrm{~d}, J_{\mathrm{C}-\mathrm{F}}=258.7 \mathrm{~Hz}\right), 145.6,133.0,132.8(\mathrm{~d}$, $\left.J_{\mathrm{C}-\mathrm{F}}=4.6 \mathrm{~Hz}\right), 132.2\left(\mathrm{~d}, J_{\mathrm{C}-\mathrm{F}}=12.1 \mathrm{~Hz}\right), 130.6\left(\mathrm{q}, J_{\mathrm{C}-\mathrm{F}}=32.4 \mathrm{~Hz}\right), 126.7(2 \mathrm{C}), 125.9\left(\mathrm{q}, J_{\mathrm{C}-\mathrm{F}}=\right.$ $11.6 \mathrm{~Hz}, 2 \mathrm{C}), 125.4\left(\mathrm{~d}, J_{\mathrm{C}-\mathrm{F}}=4.6 \mathrm{~Hz}\right), 124.1\left(\mathrm{q}, J_{\mathrm{C}-\mathrm{F}}=273.3 \mathrm{~Hz}\right), 113.8,101.8\left(\mathrm{~d}, J_{\mathrm{C}-\mathrm{F}}=15.3\right.$ $\mathrm{Hz}), 68.8\left(\mathrm{~d}, J_{\mathrm{C}-\mathrm{F}}=2.8 \mathrm{~Hz}\right)$.

${ }^{19}$ F NMR (282 MHz, CDCl $): \delta[p p m]=-62.62(\mathrm{~s}, 3 \mathrm{~F}),-111.78(\mathrm{t}, J=6.5 \mathrm{~Hz}, 1 \mathrm{~F})$.

HRMS (ESI-TOF) m/z: [M+H-H $\left.\mathrm{H}_{2} \mathrm{O}\right]^{+}$Calcd for $\mathrm{C}_{15} \mathrm{H}_{8} \mathrm{~F}_{4} \mathrm{~N} 278.0587$; Found 278.0585.

\section{2-Fluoro-3-(hydroxy(phenyl)methyl)benzonitrile (3e)}<smiles>N#Cc1cccc(C(O)c2ccccc2)c1F</smiles>

2-fluoro-3-(hydroxy(phenyl)methyl)benzonitrile was obtained from $\mathbf{1 b}$ via GMA using TMPMgCl $\mathrm{LiCl}(1 \mathrm{M}, 1.3$ equiv., $0.65 \mathrm{~mL}$ ) and benzaldehyde (1.1 equiv., $0.55 \mathrm{mmol}, 56 \mu \mathrm{L}$ ). Purification via column chromatography (Hexanes/AcOEt 8:2) afforded the product as a yellow oil (0.40 mmol, $90 \mathrm{mg}, 79 \%)$.

${ }^{1}$ H-NMR (400 MHz, CDCl3): $\delta[\mathbf{p p m}]=\delta 7.90-7.86(\mathrm{~m}, 1 \mathrm{H}), 7.51(\mathrm{ddd}, J=7.7,6.0,1.8 \mathrm{~Hz}$, $1 \mathrm{H}), 7.38-7.25(\mathrm{~m}, 6 \mathrm{H}), 6.13(\mathrm{~s}, 1 \mathrm{H}), 2.67(\mathrm{~s}, 1 \mathrm{H})$. 
${ }^{13} \mathbf{C}\left\{{ }^{1} \mathbf{H}\right\}$ NMR (101 MHz, CDCl $): \delta[\mathbf{p p m}]=160.2\left(\mathrm{~d}, J_{\mathrm{C}-\mathrm{F}}=258.9 \mathrm{~Hz}\right), 141.8,132.9\left(\mathrm{~d}, J_{\mathrm{C}-\mathrm{F}}=\right.$ $10.6 \mathrm{~Hz}), 132.8\left(\mathrm{~d}, J_{\mathrm{C}-\mathrm{F}}=4.5 \mathrm{~Hz}\right), 132.5,128.9(2 \mathrm{C}), 128.5,126.5(2 \mathrm{C}), 125.1\left(\mathrm{~d}, J_{\mathrm{C}-\mathrm{F}}=4.2 \mathrm{~Hz}\right)$, 114.0, $101.5\left(\mathrm{~d}, J_{\mathrm{C}-\mathrm{F}}=15.9 \mathrm{~Hz}\right), 69.5\left(\mathrm{~d}, J_{\mathrm{C}-\mathrm{F}}=2.9 \mathrm{~Hz}\right)$.

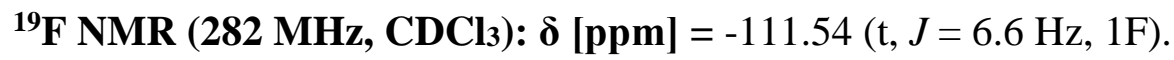

HRMS (ESI-TOF) m/z: [M+H-H $\left.{ }_{2} \mathrm{O}\right]^{+}$Calcd for $\mathrm{C}_{14} \mathrm{H}_{9} \mathrm{FN} 210.0714$; Found 210.0711.

\section{2-Fluoro-3-(hydroxy(p-tolyl)methyl)benzonitrile (3f)}<smiles>Cc1ccc(C(O)c2cccc(C#N)c2F)cc1</smiles>

2-fluoro-3-(hydroxy(phenyl)methyl)benzonitrile was obtained from $\mathbf{1 b}$ via GMA using TMPMgCl$\cdot \mathrm{LiCl}$ (1 M, 1.3 equiv., $0.65 \mathrm{~mL}$ ) and 4-methylbenzaldehyde (1.1 equiv., $0.55 \mathrm{mmol}$, $65 \mu \mathrm{L}$ ). Purification via column chromatography (Hexanes/AcOEt 8:2) afforded the product as a white solid (0.47 mmol, $113.4 \mathrm{mg}, 94 \%)$.

Mp: $76-79^{\circ} \mathrm{C}$.

${ }^{1}$ H-NMR (500 MHz, CDCl3): $\boldsymbol{\delta}$ [ppm] = $7.91-7.88(\mathrm{~m}, 1 \mathrm{H}), 7.52-7.49$ (m, 1H), 7.28 - 7.24 $(\mathrm{m}, 3 \mathrm{H}), 7.16(\mathrm{~d}, J=7.9 \mathrm{~Hz}, 2 \mathrm{H}), 6.09(\mathrm{~s}, 1 \mathrm{H}), 2.54(\mathrm{~s}, 1 \mathrm{H}), 2.33(\mathrm{~s}, 3 \mathrm{H})$.

${ }^{13} \mathbf{C}\left\{{ }^{1} \mathbf{H}\right\}$ NMR (126 MHz, CDCl 3$): \delta[\mathbf{p p m}]=160.2\left(\mathrm{~d}, J_{\mathrm{C}-\mathrm{F}}=259.0 \mathrm{~Hz}\right), 139.0,138.4,133.0(\mathrm{~d}$, $\left.J_{\mathrm{C}-\mathrm{F}}=11,9 \mathrm{~Hz}\right), 132.7\left(\mathrm{~d}, J_{\mathrm{C}-\mathrm{F}}=5.0 \mathrm{~Hz}\right), 132.4,129.6(2 \mathrm{C}), 126.4(2 \mathrm{C}), 125.0\left(\mathrm{~d}, J_{\mathrm{C}-\mathrm{F}}=4.3 \mathrm{~Hz}\right)$, 114.0, 101.6 (d, $\left.J_{\mathrm{C}-\mathrm{F}}=15.7 \mathrm{~Hz}\right), 69.5\left(\mathrm{~d}, J_{\mathrm{C}-\mathrm{F}}=2.8 \mathrm{~Hz}\right), 21.2$.

${ }^{19}$ F NMR (282 MHz, CDCl 3$): \delta[p p m]=-111.52(\mathrm{t}, J=6.6 \mathrm{~Hz}, 1 \mathrm{~F})$.

HRMS (ESI-TOF) m/z: [M+H-H $\left.{ }_{2} \mathrm{O}\right]^{+}$Calcd for $\mathrm{C}_{15} \mathrm{H}_{11} \mathrm{FN} 224.0870$; Found 224.0869.

\section{2-Fluoro-3-(furan-2-yl(hydroxy)methyl)benzonitrile (3g)}<smiles>N#Cc1cccc(C(O)c2ccco2)c1F</smiles>

2-fluoro-3-(furan-2-yl(hydroxy)methyl)benzonitrile was obtained from 1b via GMA using TMPMgCl $\mathrm{LiCl}$ (1 M, 1.3 equiv., $0.65 \mathrm{~mL}$ ) and 2-furaldehyde (1.1 equiv., $0.55 \mathrm{mmol}, 46 \mu \mathrm{L}$ ). 
Purification via column chromatography (Hexanes/AcOEt 7:3) afforded the product as an orange oil (0.47 mmol, $103.2 \mathrm{mg}, 94 \%)$.

${ }^{1}$ H-NMR (400 MHz, CDCl3): $\boldsymbol{\delta}[\mathbf{p p m}]=7.94-7.90(\mathrm{~m}, 1 \mathrm{H}), 7.59$ (ddd, $J=7.8,6.0,1.8 \mathrm{~Hz}$, $1 \mathrm{H}), 7.39$ (dd, $J=1.9,0.9 \mathrm{~Hz}, 1 \mathrm{H}), 7.31(\mathrm{t}, J=7.8 \mathrm{~Hz}, 1 \mathrm{H}), 6.34(\mathrm{dd}, J=3.3,1.9 \mathrm{~Hz}, 1 \mathrm{H}), 6.17$ $(\mathrm{d}, J=3.3 \mathrm{~Hz}, 1 \mathrm{H}), 6.14(\mathrm{~s}, 1 \mathrm{H}), 2.86(\mathrm{~s}, 1 \mathrm{H})$.

${ }^{13} \mathbf{C}\left\{{ }^{1} \mathbf{H}\right\}$ NMR (101 MHz, CDCl3): $\boldsymbol{\delta}[\mathbf{p p m}]=160.3\left(\mathrm{~d}, J_{\mathrm{C}-\mathrm{F}}=259.7 \mathrm{~Hz}\right), 153.6,143.2,133.5(\mathrm{~d}$, $\left.J_{\mathrm{C}-\mathrm{F}}=4,7 \mathrm{~Hz}\right), 133.1,129.9\left(\mathrm{~d}, J_{\mathrm{C}-\mathrm{F}}=11.6 \mathrm{~Hz}\right), 125.1\left(\mathrm{~d}, J_{\mathrm{C}-\mathrm{F}}=3.9 \mathrm{~Hz}\right), 113.9,110.6,108.1,101.6$ $\left(\mathrm{d}, J_{\mathrm{C}-\mathrm{F}}=15.3 \mathrm{~Hz}\right), 63.4\left(\mathrm{~d}, J_{\mathrm{C}-\mathrm{F}}=3.3 \mathrm{~Hz}\right)$.

${ }^{19}$ F NMR (282 MHz, CDCl3): $\delta[\mathbf{p p m}]=-112.03(\mathrm{t}, J=6.5 \mathrm{~Hz}, 1 \mathrm{~F})$.

HRMS (ESI-TOF) m/z: [M+H-H $\left.\mathrm{H}_{2} \mathrm{O}\right]^{+}$Calcd for $\mathrm{C}_{12} \mathrm{H}_{7} \mathrm{FNO} 200.0506$; Found 200.0504.

\section{3-((3-Chlorophenyl)(hydroxy)methyl)-2-fluorobenzonitrile (3h)}

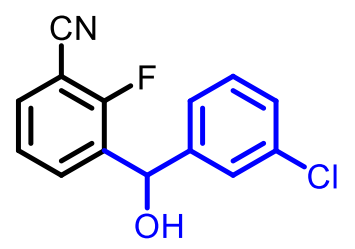

3-((3-chlorophenyl)(hydroxy)methyl)-2-fluorobenzonitrile was obtained from 1b via GMA using TMPMgCl-LiCl (1 M, 1.3 equiv., $0.65 \mathrm{~mL}$ ) and 3-chlorobenzaldehyde (1.1 equiv., 0.55 mmol, $62 \mu \mathrm{L}$ ). Purification via column chromatography (Hexanes/AcOEt 8:2) afforded the product as a yellow oil (0.40 mmol, $106 \mathrm{mg}, 81 \%)$.

${ }^{1}$ H-NMR (500 MHz, CDCl3): $\delta$ [ppm] = 7.86-7.82 (m, 1H), $7.54(\mathrm{ddd}, J=7.8,6.0,1.8 \mathrm{~Hz}$, 1H), $7.38(\mathrm{~s}, 1 \mathrm{H}), 7.31-7.24(\mathrm{~m}, 4 \mathrm{H}), 6.11(\mathrm{~s}, 1 \mathrm{H}), 2.79(\mathrm{~s}, 1 \mathrm{H})$.

${ }^{13} \mathbf{C}\left\{{ }^{1} \mathbf{H}\right\}$ NMR (126 MHz, $\left.\mathbf{C D C l}_{3}\right): \delta$ [ppm] = $160.1\left(\mathrm{~d}, J_{\mathrm{C}-\mathrm{F}}=259.1 \mathrm{~Hz}\right), 143.8,134.9,132.9$, $132.8\left(\mathrm{~d}, J_{\mathrm{C}-\mathrm{F}}=4.7 \mathrm{~Hz}\right), 132.3\left(\mathrm{~d}, J_{\mathrm{C}-\mathrm{F}}=12.0 \mathrm{~Hz}\right), 130.2,128.6,126.6,125.3\left(\mathrm{~d}, J_{\mathrm{C}-\mathrm{F}}=4.4 \mathrm{~Hz}\right)$, 124.6, 113.8, $101.7\left(\mathrm{~d}, J_{\mathrm{C}-\mathrm{F}}=15.9 \mathrm{~Hz}\right), 68.8\left(\mathrm{~d}, J_{\mathrm{C}-\mathrm{F}}=2.9 \mathrm{~Hz}\right)$.

${ }^{19}$ F NMR (282 MHz, CDCl3): $\delta[p p m]=-111.67(\mathrm{t}, J=6.5 \mathrm{~Hz}, 1 \mathrm{~F})$.

HRMS (ESI-TOF) m/z: [M+H-H $\left.{ }_{2} \mathrm{O}\right]^{+}$Calcd for $\mathrm{C}_{14} \mathrm{H}_{8} \mathrm{ClFN} 244.0324$; Found 244.0327.

\section{3-((3-Bromophenyl)(hydroxy)methyl)-4-fluorobenzonitrile (3i)}




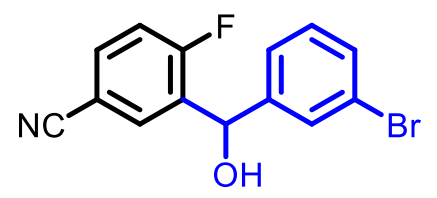

3-((3-bromophenyl)(hydroxy)methyl)-4-fluorobenzonitrile was obtained from 1c via GMA using TMPMgCl $\mathrm{LiCl}(1 \mathrm{M}, 1.3$ equiv., $0.65 \mathrm{~mL}$ ) and 3-bromobenzaldehyde (1.1 equiv., $0.55 \mathrm{mmol}$, $64 \mu \mathrm{L}$ ). Purification via column chromatography (Hexanes/AcOEt 8:2) afforded the product as a white solid (0.41 mmol, $127.0 \mathrm{mg}, 83 \%)$.

Mp: $111-114^{\circ} \mathrm{C}$.

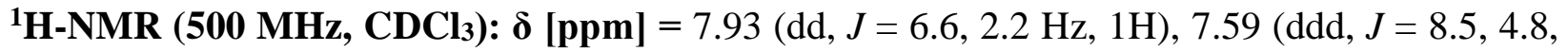
$2.2 \mathrm{~Hz}, 1 \mathrm{H}), 7.53$ (s, 1H), $7.44-7.42(\mathrm{~m}, 1 \mathrm{H}), 7.31(\mathrm{~d}, J=7.7 \mathrm{~Hz}, 1 \mathrm{H}), 7.23$ (t, $J=7.8 \mathrm{~Hz}, 1 \mathrm{H})$, 7.13 (t-like, $J=8.5 \mathrm{~Hz}, 1 \mathrm{H}), 6.07$ (s, 1H), 2.39 (bs, 1H).

${ }^{13} \mathbf{C}\left\{{ }^{1} \mathbf{H}\right\}$ NMR $\left(\mathbf{1 2 6} \mathbf{M H z}, \mathbf{C D C l}_{3}\right): \boldsymbol{\delta}[\mathbf{p p m}]=162.0\left(\mathrm{~d}, J_{\mathrm{C}-\mathrm{F}}=256.8 \mathrm{~Hz}\right), 144.0,133.9\left(\mathrm{~d}, J_{\mathrm{C}-\mathrm{F}}\right.$ $=9.7 \mathrm{~Hz}), 132.7\left(\mathrm{~d}, J_{\mathrm{C}-\mathrm{F}}=14.5 \mathrm{~Hz}\right), 132.3\left(\mathrm{~d}, J_{\mathrm{C}-\mathrm{F}}=5.5 \mathrm{~Hz}\right), 131.6,130.5,129.6,125.1,123.1$, $118.1,117.0\left(\mathrm{~d}, J_{\mathrm{C}-\mathrm{F}}=23.3 \mathrm{~Hz}\right), 109.1\left(\mathrm{~d}, J_{\mathrm{C}-\mathrm{F}}=3.8 \mathrm{~Hz}\right), 68.9\left(\mathrm{~d}, J_{\mathrm{C}-\mathrm{F}}=2.7 \mathrm{~Hz}\right)$.

${ }^{19}$ F NMR (282 MHz, CDCl 3$): \delta[\mathbf{p p m}]=-107.77--107.85(\mathrm{~m}, 1 \mathrm{~F})$.

HRMS (ESI-TOF) m/z: $[\mathrm{M}+\mathrm{H}]^{+}$Calcd for $\mathrm{C}_{14} \mathrm{H}_{10} \mathrm{BrFNO} 305.9925$; Found 305.9921.

10. 4-Fluoro-3-(hydroxy(thiophen-2-yl)methyl)benzonitrile (3j)<smiles>N#Cc1ccc(F)c(C(O)c2cccs2)c1</smiles>

4-fluoro-3-(hydroxy(thiophen-2-yl)methyl)benzonitrile was obtained from 1c via GMA using TMPMgCl $\mathrm{LiCl}(1 \mathrm{M}, 1.3$ equiv., $0.65 \mathrm{~mL})$ and 2-thiophenecarboxaldehyde (1.1 equiv., 0.55 mmol, $51 \mu \mathrm{L}$ ). Purification via column chromatography (Hexanes/AcOEt 8:2) afforded the product as a yellow solid (0.44 mmol, $104.0 \mathrm{mg}, 89 \%)$. CAS: 2237249-90-8.

Mp: $60-62{ }^{\circ} \mathrm{C}$.

${ }^{1} \mathbf{H}-\mathrm{NMR}(\mathbf{5 0 0} \mathrm{MHz}, \mathbf{C D C l}): \boldsymbol{\delta}[\mathbf{p p m}]=8.01(\mathrm{dd}, J=6.7,2.2 \mathrm{~Hz}, 1 \mathrm{H}), 7.61(\mathrm{ddd}, J=8.5,4.7$, $2.2 \mathrm{~Hz}, 1 \mathrm{H}), 7.30(\mathrm{dd}, J=4.2,2.2 \mathrm{~Hz}, 1 \mathrm{H}), 7.14(\mathrm{dd}, J=9.4,8.5 \mathrm{~Hz}, 1 \mathrm{H}), 6.97-6.96(\mathrm{~m}, 2 \mathrm{H})$, 6.34 (s, 1H), 2.85 (s, 1H). 
${ }^{13} \mathbf{C}\left\{{ }^{1} \mathbf{H}\right\}$ NMR (126 MHz, CDCl 3$): \delta$ [ppm] $=162.1\left(\mathrm{~d}, J_{\mathrm{C}-\mathrm{F}}=257.0 \mathrm{~Hz}\right), 145.6,133.9\left(\mathrm{~d}, J_{\mathrm{C}-\mathrm{F}}\right.$ =9.6 Hz), $132.8\left(\mathrm{~d}, J_{\mathrm{C}-\mathrm{F}}=14.3 \mathrm{~Hz}\right), 132.2\left(\mathrm{~d}, J_{\mathrm{C}-\mathrm{F}}=5.3 \mathrm{~Hz}\right), 127.1,126.2,125.4,118.1,117.0(\mathrm{~d}$, $\left.J_{\mathrm{C}-\mathrm{F}}=23.2 \mathrm{~Hz}\right), 109.0\left(\mathrm{~d}, J_{\mathrm{C}-\mathrm{F}}=3.9 \mathrm{~Hz}\right), 65.6\left(\mathrm{~d}, J_{\mathrm{C}-\mathrm{F}}=3.7 \mathrm{~Hz}\right)$.

${ }^{19}$ F NMR (282 MHz, CDCl 3$): \delta[p p m]=-108.19--108.27(\mathrm{~m}, 1 \mathrm{~F})$.

HRMS (ESI-TOF) m/z: [M-H] Calcd for $\mathrm{C}_{12} \mathrm{H}_{7}$ FNOS 232.0238; Found 232.0235.

The reported characterization data is in accordance with the literature. ${ }^{4}$

\section{4-Fluoro-3-((4-fluorophenyl)(hydroxy)methyl)benzonitrile (3k)}<smiles>N#Cc1ccc(F)c(C(O)c2ccc(F)cc2)c1</smiles>

4-fluoro-3-((4-fluorophenyl)(hydroxy)methyl)benzonitrile was obtained from 1c via GMA using TMPMgCl-LiCl (1 M, 1.3 equiv., $0.65 \mathrm{~mL}$ ) and 4-fluorobenzaldehyde (1.1 equiv., 0.55 mmol, 59 $\mu \mathrm{L}$ ). Purification via column chromatography (Hexanes/AcOEt 8:2) afforded the product as a white solid (0.40 mmol, $98.1 \mathrm{mg}, 80 \%)$. CAS: 1519447-09-6.

Mp: $82-84{ }^{\circ} \mathrm{C}$.

${ }^{1}$ H-NMR (500 MHz, CDCl3): $\boldsymbol{\delta}$ [ppm] $=7.96(\mathrm{dd}, J=6.8,2.2 \mathrm{~Hz}, 1 \mathrm{H}), 7.58(\mathrm{ddd}, J=8.5,4.7$, $2.2 \mathrm{~Hz}, 1 \mathrm{H}), 7.35(\mathrm{dd}, J=8.5,5.4 \mathrm{~Hz}, 2 \mathrm{H}), 7.11(\mathrm{dd}, J=9.7,8.5 \mathrm{~Hz}, 1 \mathrm{H}), 7.06-7.02(\mathrm{~m}, 2 \mathrm{H})$, $6.09(\mathrm{~s}, 1 \mathrm{H}), 2.61(\mathrm{~d}, J=2.6 \mathrm{~Hz}, 1 \mathrm{H})$.

${ }^{13} \mathbf{C}\left\{{ }^{1} \mathbf{H}\right\}$ NMR (126 MHz, CDCl3): $\delta[\mathbf{p p m}]=162.7\left(\mathrm{~d}, J_{\mathrm{C}-\mathrm{F}}=247.4 \mathrm{~Hz}\right), 162.0\left(\mathrm{~d}, J_{\mathrm{C}-\mathrm{F}}=256.4\right.$ $\mathrm{Hz}), 137.6\left(\mathrm{~d}, J_{\mathrm{C}-\mathrm{F}}=3.1 \mathrm{~Hz}\right), 133.7\left(\mathrm{~d}, J_{\mathrm{C}-\mathrm{F}}=9.7 \mathrm{~Hz}\right), 133.2\left(\mathrm{~d}, J_{\mathrm{C}-\mathrm{F}}=14.5 \mathrm{~Hz}\right), 132.1\left(\mathrm{~d}, J_{\mathrm{C}-\mathrm{F}}=5.6\right.$ $\mathrm{Hz}), 128.4\left(\mathrm{~d}, J_{\mathrm{C}-\mathrm{F}}=8.3 \mathrm{~Hz}, 2 \mathrm{C}\right), 118.2,117.6$, (d, $\left.J_{\mathrm{C}-\mathrm{F}}=23.1 \mathrm{~Hz}\right), 115.9\left(\mathrm{~d}, J_{\mathrm{C}-\mathrm{F}}=21.9 \mathrm{~Hz}, 2 \mathrm{C}\right)$, $109.0\left(\mathrm{~d}, J_{\mathrm{C}-\mathrm{F}}=3.9 \mathrm{~Hz}\right), 69.0\left(\mathrm{~d}, J_{\mathrm{C}-\mathrm{F}}=2.8 \mathrm{~Hz}\right)$.

${ }^{19}$ F NMR (282 MHz, CDCl3): $\delta$ [ppm] = -107.84 - -107.92 (m, 1F), -113.27 - -113.37 (m, 1F).

HRMS (ESI-TOF) m/z: [M+Na] ${ }^{+}$Calcd for $\mathrm{C}_{14} \mathrm{H}_{9} \mathrm{~F}_{2} \mathrm{NNaO} 268.0544$; Found 268.0546.

\section{4-Fluoro-3-(hydroxy(phenyl)methyl)benzonitrile (3l)}

\footnotetext{
4 Benischke, A. D.; Anthore-Dalion, L.; Kohl, F.; Knochel, P. Synthesis of Polyfunctionalized Triaryllanthanum Reagents by Using $\mathrm{Ph}_{3} \mathrm{La}$ and Related Species as Exchange Reagents. Chem. - Eur. J. 2018, 24 (43), 11103-11109.
} 


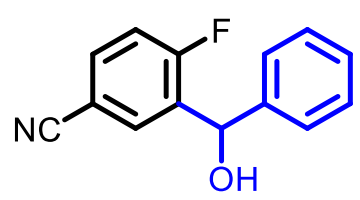

4-fluoro-3-(hydroxy(phenyl)methyl)benzonitrile was obtained from 1c via GMA using TMPMgCl $\mathrm{LiCl}(1 \mathrm{M}, 1.3$ equiv., $0.65 \mathrm{~mL})$ and benzaldehyde (1.1 equiv., $0.55 \mathrm{mmol}, 56 \mu \mathrm{L})$. Purification via column chromatography (Hexanes/AcOEt 8:2) afforded the product as a white solid (0.36 mmol, $83 \mathrm{mg}, 73 \%)$. CAS: 1499388-65-6.

Mp: $101-103^{\circ} \mathrm{C}$.

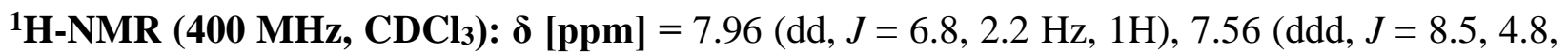
$2.2 \mathrm{~Hz}, 1 \mathrm{H}), 7.39-7.35$ (m, 4H), $7.35-7.29$ (m, 1H), 7.10 (dd, $J=9.6,8.5 \mathrm{~Hz}, 1 \mathrm{H}), 6.10$ (s, $1 \mathrm{H}), 2.40(\mathrm{~s}, 1 \mathrm{H})$.

${ }^{13} \mathbf{C}\left\{{ }^{1} \mathbf{H}\right\}$ NMR $\left(101 \mathrm{MHz}, \mathbf{C D C l}_{3}\right): \boldsymbol{\delta}[\mathbf{p p m}]=162.1\left(\mathrm{~d}, J_{\mathrm{C}-\mathrm{F}}=256.6 \mathrm{~Hz}\right), 141.7,133.5\left(\mathrm{~d}, J_{\mathrm{C}-\mathrm{F}}\right.$ $=9.6 \mathrm{~Hz}), 133.2\left(\mathrm{~d}, J_{\mathrm{C}-\mathrm{F}}=14.6 \mathrm{~Hz}\right), 132.2\left(\mathrm{~d}, J_{\mathrm{C}-\mathrm{F}}=5.6 \mathrm{~Hz}\right), 129.0(2 \mathrm{C}), 128.5,126.5(2 \mathrm{C}), 118.3$, $116.9\left(\mathrm{~d}, J_{\mathrm{C}-\mathrm{F}}=23.3 \mathrm{~Hz}\right), 108.8\left(\mathrm{~d}, J_{\mathrm{C}-\mathrm{F}}=3.8 \mathrm{~Hz}\right), 69.6\left(\mathrm{~d}, J_{\mathrm{C}-\mathrm{F}}=2.7 \mathrm{~Hz}\right)$.

${ }^{19}$ F NMR (282 MHz, CDCl 3$): \delta[\mathbf{p p m}]=-107.68--107.75(\mathrm{~m}, 1 \mathrm{~F})$.

GC-MS (EI, 70 eV) m/z: 227.00 (100\%), 79.05 (96\%), 148.00 (68\%), 78.05 (37\%), 77.00 $(37 \%)$.

HRMS (ESI-TOF) m/z: [M+FA-H] ${ }^{-}$Calcd for $\mathrm{C}_{15} \mathrm{H}_{11} \mathrm{FNO}_{3}$ 272.0728; Found 272.0701.

The reported characterization data is in accordance with the literature. ${ }^{5}$

\section{4-Fluoro-3-(p-tolyl)isobenzofuran-1(3H)-imine (3m)}

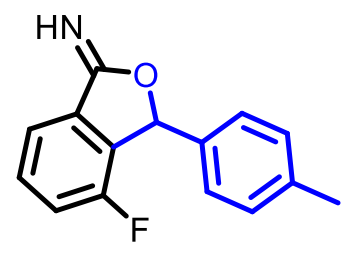

4-fluoro-3-( $p$-tolyl)isobenzofuran-1(3H)-imine was obtained from 1a via GMA using TMPMgCl $\mathrm{LiCl}(1 \mathrm{M}, 1.2$ equiv., $0.6 \mathrm{~mL}$ ) and 4-methylbenzaldehyde (1.1 equiv., $0.55 \mathrm{mmol}, 65$ $\mu \mathrm{L}$ ). Purification via column chromatography (Hexanes/AcOEt 8:2) afforded the product as a white solid (0.31 mmol, $75 \mathrm{mg}, 62 \%)$.

5 Weidmann, N.; Ketels, M.; Knochel, P. Sodiation of Arenes and Heteroarenes in Continuous Flow. Angew. Chem. Int. Ed. 2018, 57 (33), 10748-10751. 
Mp: $121-125^{\circ} \mathrm{C}$.

${ }^{1}$ H-NMR (500 MHz, CDCl3): $\boldsymbol{\delta}[\mathbf{p p m}]=7.74(\mathrm{~d}, J=6.9 \mathrm{~Hz}, 1 \mathrm{H}), 7.49(\mathrm{td}, J=7.9,4.5 \mathrm{~Hz}, 1 \mathrm{H})$, $7.20-7.16(\mathrm{~m}, 5 \mathrm{H}), 6.46(\mathrm{~s}, 1 \mathrm{H}), 2.35(\mathrm{~s}, 3 \mathrm{H})$.

${ }^{13} \mathbf{C}\left\{{ }^{1} \mathbf{H}\right\}$ NMR (126 MHz, CDCl 3$): \delta[\mathbf{p p m}]=157.2\left(\mathrm{~d}, J_{\mathrm{C}-\mathrm{F}}=251.4 \mathrm{~Hz}\right), 139.4,133.6,133.5(\mathrm{~d}$, $\left.J_{\mathrm{C}-\mathrm{F}}=17.8 \mathrm{~Hz}\right), 131.5\left(\mathrm{~d}, J_{\mathrm{C}-\mathrm{F}}=6.3 \mathrm{~Hz}\right), 129.6(2 \mathrm{C}), 127.1(2 \mathrm{C}), 120.0(2 \mathrm{C}), 119.1\left(\mathrm{~d}, J_{\mathrm{C}-\mathrm{F}}=19,6\right.$ $\mathrm{Hz}, 2 \mathrm{C}), 82.9,21.3$.

${ }^{19}$ F NMR (282 MHz, CDCl3): $\delta[\mathbf{p p m}]=-118.29(\mathrm{dd}, J=8.8,4.6 \mathrm{~Hz}, 1 \mathrm{~F})$.

HRMS (ESI-TOF) m/z: $[\mathrm{M}+\mathrm{H}]^{+}$Calcd for $\mathrm{C}_{15} \mathrm{H}_{12} \mathrm{FO}_{2}$ 243.0816; Found 243.0815. * Under the analysis conditions ( $\mathrm{MeCN}: \mathrm{H}_{2} \mathrm{O}$ mixture, formic acid) the 3-substituted-isobenzofuran-1(3H)imine was converted into the respective isobenzofuran-1(3H)-one. The hydrolysis under acidic conditions is already mentioned in the literature. ${ }^{6}$

\section{3-(2-Bromophenyl)-4-fluoroisobenzofuran-1(3H)-imine (3n)}

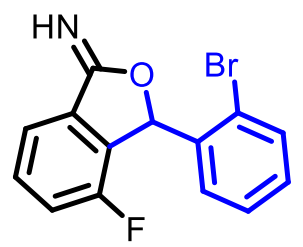

3-(2-bromophenyl)-4-fluoroisobenzofuran-1(3H)-imine was obtained from 1a via GMA using TMPMgCl $\mathrm{LiCl}(1 \mathrm{M}, 1.2$ equiv., $0.6 \mathrm{~mL}$ ) and 2-bromobenzaldehyde (1.1 equiv., $0.55 \mathrm{mmol}, 64$ $\mu \mathrm{L}$ ). Purification via column chromatography (Hexanes/AcOEt 8:2) afforded the product as a yellow solid (0.29 mmol, $89 \mathrm{mg}, 58 \%)$.

Mp: $112-115^{\circ} \mathrm{C}$.

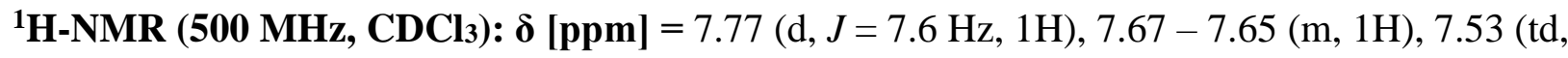
$J=7.9,4.5 \mathrm{~Hz}, 1 \mathrm{H}), 7.27-7.21(\mathrm{~m}, 3 \mathrm{H}), 6.98(\mathrm{~s}, 1 \mathrm{H}), 6.95(\mathrm{dd}, J=7.2,2.5 \mathrm{~Hz}, 1 \mathrm{H})$.

${ }^{13} \mathbf{C}\left\{{ }^{1} \mathbf{H}\right\}$ NMR (126 MHz, CDCl3): $\delta[\mathbf{p p m}]=157.1\left(\mathrm{~d}, J_{\mathrm{C}-\mathrm{F}}=253.0 \mathrm{~Hz}\right), 135.4,133.6,132.6(\mathrm{~d}$, $\left.J_{\mathrm{C}-\mathrm{F}}=17.9 \mathrm{~Hz}\right), 131.9\left(\mathrm{~d}, J_{\mathrm{C}-\mathrm{F}}=6.6 \mathrm{~Hz}\right), 131.0,128.9,128.1,124.3,120.2,120.1,119.3\left(\mathrm{~d}, J_{\mathrm{C}-\mathrm{F}}\right.$ $=19.1 \mathrm{~Hz}, 2 \mathrm{C}), 81.9$.

\footnotetext{
${ }^{6}$ Kobayashi, K.; Matsumoto, K.; Konishi, H. An Efficient Synthesis of 3-Substituted 3H-Isobenzofuran-1-Ylidenamines by the Reaction of 2-Cyanobenzaldehydes with Organolithiums and Their Conversion into Isobenzofuran-1(3H)-Ones. HETEROCYCLES 2011, 83 (1), 99.
} 
HRMS (ESI-TOF) m/z: $[\mathrm{M}+\mathrm{H}]^{+}$Calcd for $\mathrm{C}_{14} \mathrm{H}_{9} \mathrm{BrFO}_{2}$ 306.9765; Found 306.9765. * Under the analysis conditions ( $\mathrm{MeCN}: \mathrm{H}_{2} \mathrm{O}$ mixture, formic acid) the 3-substituted-isobenzofuran$1(3 H)$-imine was converted into the respective isobenzofuran-1(3H)-one. The hydrolysis under acidic conditions is already mentioned in the literature. ${ }^{6}$

\section{3-(2-Chlorophenyl)-4-fluoroisobenzofuran-1(3H)-imine (3o)}

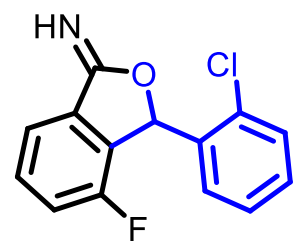

3-(2-chlorophenyl)-4-fluoroisobenzofuran-1(3H)-imine was obtained from 1a via GMA using TMPMgCl $\mathrm{LiCl}$ (1 M, 1.2 equiv., $0.6 \mathrm{~mL}$ ) and 2-chlorobenzaldehyde (1.1 equiv., $0.55 \mathrm{mmol}, 62$ $\mu \mathrm{L}$ ). Purification via column chromatography (Hexanes/AcOEt 8:2) afforded the product as a white solid (0.24 mmol, $64 \mathrm{mg}, 49 \%)$.

Yield: 49\% (white solid, $64 \mathrm{mg}$ ).

Mp: $110-113^{\circ} \mathrm{C}$.

${ }^{1} \mathrm{H}-\mathrm{NMR}\left(\mathbf{5 0 0} \mathrm{MHz}, \mathbf{C D C l}_{3}\right): \boldsymbol{\delta}[\mathbf{p p m}]=7.76(\mathrm{~d}, J=7.6 \mathrm{~Hz}, 1 \mathrm{H}), 7.52(\mathrm{td}, J=8.0,4.5 \mathrm{~Hz}, 1 \mathrm{H})$, $7.46(\mathrm{~d}, J=8.0 \mathrm{~Hz}, 1 \mathrm{H}), 7.32-7.29(\mathrm{~m}, 1 \mathrm{H}), 7.23-7.20(\mathrm{~m}, 2 \mathrm{H}), 6.99-6.97(\mathrm{~m}, 2 \mathrm{H})$.

${ }^{13} \mathbf{C}\left\{{ }^{1} \mathbf{H}\right\}$ NMR (126 MHz, CDCl 3$): \delta[\mathbf{p p m}]=157.1\left(\mathrm{~d}, J_{\mathrm{C}-\mathrm{F}}=252.1 \mathrm{~Hz}\right), 134.3,133.8,132.5(\mathrm{~d}$, $\left.J_{\mathrm{C}-\mathrm{F}}=17.7 \mathrm{~Hz}\right), 131.9\left(\mathrm{~d}, J_{\mathrm{C}-\mathrm{F}}=6.6 \mathrm{~Hz}\right), 130.7,130.3,128.7,127.4,120.1(2 \mathrm{C}), 119.2\left(\mathrm{~d}, J_{\mathrm{C}-\mathrm{F}}\right.$ $=19.4 \mathrm{~Hz}, 2 \mathrm{C}), 79.5$.

HRMS (ESI-TOF) m/z: $[\mathrm{M}+\mathrm{H}]^{+}$Calcd for $\mathrm{C}_{14} \mathrm{H}_{9} \mathrm{ClFO}_{2}$ 263.0270; Found 263.0266. *Under the analysis conditions (MeCN: $\mathrm{H}_{2} \mathrm{O}$ mixture, formic acid) the 3-substituted-isobenzofuran-1(3H)imine was converted into the respective isobenzofuran-1(3H)-one. The hydrolysis under acidic conditions is already mentioned in the literature. ${ }^{6}$

\section{2-Fluoro-3-formylbenzonitrile (3p)}<smiles>N#Cc1cccc(C=O)c1F</smiles> 
2-fluoro-3-formylbenzonitrile was obtained from 1b via GMA using TMPMgCl LiCl (1 M, 1.3 equiv., $0.65 \mathrm{~mL}$ ) and $N, N$-dimethylformamide (2.0 equiv., $1.0 \mathrm{mmol}, 77 \mu \mathrm{L})$. Purification via column chromatography (Hexanes/AcOEt 8:2) afforded the product as a yellow solid (0.29 mmol, 44 mg, 59\%). CAS: 1261823-31-7.

Mp: $46-48^{\circ} \mathrm{C}$.

${ }^{1}$ H-NMR (400 MHz, CDCl 3$): \delta[p p m]=10.36(\mathrm{~s}, 1 \mathrm{H}), 8.13(\mathrm{ddd}, J=7.9,6.7,1.9 \mathrm{~Hz}, 1 \mathrm{H})$, 7.90 (ddd, $J=7.8,6.1,1.9 \mathrm{~Hz}, 1 \mathrm{H}), 7.44$ (t-like, $J=7,8 \mathrm{~Hz}, 1 \mathrm{H})$.

${ }^{13} \mathbf{C}\left\{{ }^{1} \mathbf{H}\right\}$ NMR (101 MHz, CDCl$): \delta[\mathbf{p p m}]=184.9\left(\mathrm{~d}, J_{\mathrm{C}-\mathrm{F}}=6.3 \mathrm{~Hz}\right), 164.9\left(\mathrm{~d}, J_{\mathrm{C}-\mathrm{F}}=270.0 \mathrm{~Hz}\right)$, $139.1,133.5,125.6\left(\mathrm{~d}, J_{\mathrm{C}-\mathrm{F}}=4.6 \mathrm{~Hz}\right), 124.8\left(\mathrm{~d}, J_{\mathrm{C}-\mathrm{F}}=7.4 \mathrm{~Hz}\right), 112.7,103.2\left(\mathrm{~d}, J_{\mathrm{C}-\mathrm{F}}=14.9 \mathrm{~Hz}\right)$.

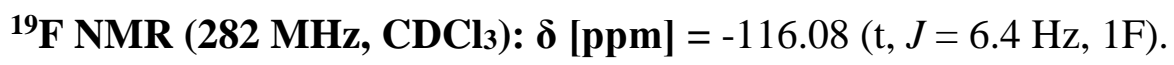

HRMS (ESI-TOF) m/z: [M+H $]^{+}$Calcd for $\mathrm{C}_{8} \mathrm{H}_{5} \mathrm{FNO} 150.0350$; Found 150.0351.

\section{4-(Dimethylamino)-3-formylbenzonitrile (3q)}<smiles>CN(C)c1ccc(C#N)cc1C=O</smiles>

4-(dimethylamino)-3-formylbenzonitrile was obtained from 1c via GMA using TMPMgCl $\mathrm{LiCl}$ (1 M, 1.3 equiv., $0.65 \mathrm{~mL})$ and $N, N$-dimethylformamide (2.0 equiv., $1.0 \mathrm{mmol}, 77 \mu \mathrm{L})$. Purification via column chromatography (Hexanes/AcOEt 8:2) afforded the product as a yellow solid (0.45 mmol, $79.3 \mathrm{mg}, 91 \%)$. CAS: 1289200-50-5.

Mp: $62-64^{\circ} \mathrm{C}$.

${ }^{1}$ H-NMR (500 MHz, CDCl3): $\boldsymbol{\delta}$ [ppm] = $9.97(\mathrm{~s}, 1 \mathrm{H}), 7.96(\mathrm{~d}, J=2.2 \mathrm{~Hz}, 1 \mathrm{H}), 7.57(\mathrm{dd}, J=$ 8.8, 2.2 Hz, 1H), 6.97 (d, $J=8.8 \mathrm{~Hz}, 1 \mathrm{H}), 3.06(\mathrm{~s}, 6 \mathrm{H})$.*

${ }^{13} \mathbf{C}\left\{{ }^{1} \mathbf{H}\right\}$ NMR (126 MHz, $\left.\mathbf{C D C l}_{3}\right): \boldsymbol{\delta}[\mathbf{p p m}]=188.2,155.9,137.9,136.6,124.4,119.0,116.9$, 101.0, $44.5(2 \mathrm{C})$.

HRMS (ESI-TOF) m/z: [M+H] ${ }^{+}$Calcd for $\mathrm{C}_{10} \mathrm{H}_{11} \mathrm{~N}_{2} \mathrm{O}$ 175.0866; Found 175.0863.

*Previously reported in the literature. ${ }^{7}$

\footnotetext{
${ }^{7}$ Bougeret, C.; Guillou, C.; Rouleau, J.; Rivollier, J.; Carniato, D. New derivatives of indole for the treatment of cancer, viral infections and lung diseases. U.S. patent US 2015/0307450 A1.
} 


\section{4-Fluoro-3-formylbenzonitrile (3r)}<smiles>N#Cc1ccc(F)c(C=O)c1</smiles>

4-fluoro-3-formylbenzonitrile was obtained from 1c via GMA using TMPMgCl-LiCl (1 M, 1.3 equiv., $0.65 \mathrm{~mL}$ ) and $N$-methylformanilide (2.0 equiv., $1.0 \mathrm{mmol}, 123 \mu \mathrm{L})$. Purification via column chromatography (Hexanes/AcOEt 9:1) afforded the product as a white solid (0.31 mmol, $46.2 \mathrm{mg}, 62 \%)$. CAS: 146137-79-3.

Yield: $62 \%$ (white solid, $46.2 \mathrm{mg}$ ).

Mp: $68-71{ }^{\circ} \mathrm{C}$.

${ }^{1}$ H-NMR (400 MHz, CDCl3): $\delta$ [ppm] = $10.34(\mathrm{~s}, 1 \mathrm{H}), 8.20(\mathrm{ddd}, J=6.3,2.3,0.4 \mathrm{~Hz}, 1 \mathrm{H})$, $7.91(\mathrm{ddd}, J=8.7,4.8,2.3 \mathrm{~Hz}, 1 \mathrm{H}), 7.38-7.33(\mathrm{~m}, 1 \mathrm{H})$.

${ }^{13} \mathbf{C}\left\{{ }^{1} \mathbf{H}\right\}$ NMR (101 MHz, CDCl3): $\delta[\mathbf{p p m}]=185.0(\mathrm{~d}, J=6.2 \mathrm{~Hz}), 166.4\left(\mathrm{~d}, J_{\mathrm{C}-\mathrm{F}}=267.5 \mathrm{~Hz}\right)$, $139.6\left(\mathrm{~d}, J_{\mathrm{C}-\mathrm{F}}=10.4 \mathrm{~Hz}\right), 133.6\left(\mathrm{~d}, J_{\mathrm{C}-\mathrm{F}}=3.3 \mathrm{~Hz}\right), 125.0\left(\mathrm{~d}, J_{\mathrm{C}-\mathrm{F}}=9.8 \mathrm{~Hz}\right), 118.5\left(\mathrm{~d}, J_{\mathrm{C}-\mathrm{F}}=22.3\right.$ $\mathrm{Hz}), 117.0,109.9\left(\mathrm{~d}, J_{\mathrm{C}-\mathrm{F}}=3.9 \mathrm{~Hz}\right)$.

${ }^{19}$ F NMR (282 MHz, CDCl 3$): \delta[p p m]=-112.19--112.27(\mathrm{~m}, 1 \mathrm{~F})$.

GC-MS (EI, 70 eV) m/z: 148.05 (100\%), 149.05 (64\%), 120.05 (36\%), 94.00 (15\%), 100.00 $(14 \%)$.

The reported characterization data is in accordance with the literature. ${ }^{8}$

\section{2-Fluoro-4'-methoxy-[1,1'-biphenyl]-3-carbonitrile (3s)}

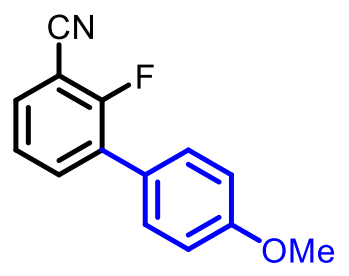

After applying GMA [TMPMgCl $\mathrm{LiCl}(1 \mathrm{M}, 1.3$ equiv., $0.65 \mathrm{~mL})$ ] for $\mathbf{1 b}$ followed by transmetalation with $\mathrm{ZnCl}_{2}$ (1 M in THF, 1.3 equiv., $0.65 \mathrm{~mL}$ ), the Negishi cross-coupling was

\footnotetext{
${ }^{8}$ Bhagwat, S. S.; Satoh, Y.; Sakata, S. T.; Buhr, C. A.; Albers, R.; Sapienza, J.; Plantevin, V.; Chao, Q.; Sahasrabudhe, K.; Ferri, R.; Narla, R. K. Indazole compounds, compositions thereof and methods of treatment therewith. U.S. patent, US 2005/0009876 A1.
} 
performed using $\mathrm{Pd}\left(\mathrm{PPh}_{3}\right)_{4}(5 \mathrm{~mol} \%, 29 \mathrm{mg})$ and 4-methoxyiodobenzene (1.2 equiv., $0.6 \mathrm{mmol}$, $140.4 \mathrm{mg}$ ) at $50{ }^{\circ} \mathrm{C}$ (oil bath). Purification via column chromatography (Hexanes/AcOEt 9:1) afforded the product as a beige solid (0.45 mmol, $102.3 \mathrm{mg}, 90 \%)$.

Mp: $106-108^{\circ} \mathrm{C}$.

${ }^{1}$ H-NMR (500 MHz, CDCl3): $\boldsymbol{\delta}$ [ppm] $=7.66$ (t-like, $\left.J=7.8 \mathrm{~Hz}, 1 \mathrm{H}\right), 7.55$ (t-like, $J=7.1 \mathrm{~Hz}$, $1 \mathrm{H}), 7.47(\mathrm{~d}, J=8.5 \mathrm{~Hz}, 2 \mathrm{H}), 7.30(\mathrm{t}, J=7.8 \mathrm{~Hz}, 1 \mathrm{H}), 7.00(\mathrm{~d}, J=8.5 \mathrm{~Hz}, 2 \mathrm{H}), 3.86(\mathrm{~s}, 3 \mathrm{H})$.

${ }^{13} \mathbf{C}\left\{{ }^{1} \mathbf{H}\right\}$ NMR (126 MHz, $\left.\mathbf{C D C l}_{3}\right): \delta[\mathbf{p p m}]=160.3\left(\mathrm{~d}, J_{\mathrm{C}-\mathrm{F}}=259.6 \mathrm{~Hz}\right), 160.1,135.5\left(\mathrm{~d}, J_{\mathrm{C}-\mathrm{F}}\right.$ $=4.1 \mathrm{~Hz}), 131.8,130.3\left(\mathrm{~d}, J_{\mathrm{C}-\mathrm{F}}=14.0 \mathrm{~Hz}\right), 130.2\left(\mathrm{~d}, J_{\mathrm{C}-\mathrm{F}}=3.1 \mathrm{~Hz}, 2 \mathrm{C}\right), 125.9,125.1\left(\mathrm{~d}, J_{\mathrm{C}-\mathrm{F}}=4.3\right.$ $\mathrm{Hz}), 114.4(2 \mathrm{C}), 114.3,102.4\left(\mathrm{~d}, J_{\mathrm{C}-\mathrm{F}}=17.0 \mathrm{~Hz}\right), 55.5$.

${ }^{19}$ F NMR (282 MHz, CDCl 3$): \delta[p p m]=-111.52(\mathrm{t}, J=6.6 \mathrm{~Hz}, 1 \mathrm{~F})$.

HRMS (ESI-TOF) m/z: [M+H $]^{+}$Calcd for $\mathrm{C}_{14} \mathrm{H}_{11} \mathrm{FNO} 228.0819$; Found 228.0818.

\section{6-Fluoro-3'-methyl-[1,1'-biphenyl]-3-carbonitrile (3t)}

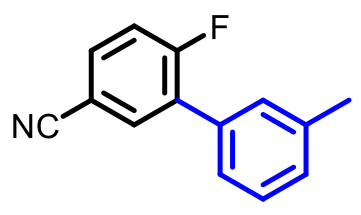

After applying GMA [TMPMgCl $\mathrm{LiCl}$ (1 M, 1.3 equiv., $0.65 \mathrm{~mL})$ ] for 1c followed by transmetalation with $\mathrm{ZnCl}_{2}(1 \mathrm{M}$ in THF, 1.3 equiv., $0.65 \mathrm{~mL})$, the Negishi cross-coupling was performed using $\mathrm{Pd}\left(\mathrm{PPh}_{3}\right)_{4}(5 \mathrm{~mol} \%, 29 \mathrm{mg})$ and 1-iodo-3-methylbenzene (1.2 equiv., 0.6 $\mathrm{mmol}, 77 \mu \mathrm{L}$ ) at $50{ }^{\circ} \mathrm{C}$ (oil bath). Purification via column chromatography (Hexanes/AcOEt 9:1) afforded the product as a white solid (0.46 mmol, $97.2 \mathrm{mg}, 92 \%)$. CAS: 1267960-92-8.

Mp: $57-59^{\circ} \mathrm{C}$.

${ }^{1}$ H-NMR (500 MHz, CDCl3): $\boldsymbol{\delta}[\mathbf{p p m}]=7.66(\mathrm{dd}, J=7.0,2.2 \mathrm{~Hz}, 1 \mathrm{H}), 7.53(\mathrm{ddd}, J=8.6,4.5$, $2.2 \mathrm{~Hz}, 1 \mathrm{H}), 7.27(\mathrm{t}, J=7.5 \mathrm{~Hz}, 1 \mathrm{H}), 7.23-7.21(\mathrm{~m}, 2 \mathrm{H}), 7.18-7.14(\mathrm{~m}, 2 \mathrm{H}), 2.33(\mathrm{~s}, 3 \mathrm{H})$.

${ }^{13} \mathbf{C}\left\{{ }^{1} \mathbf{H}\right\}$ NMR (126 MHz, CDCl $): \delta[\mathbf{p p m}]=162.2\left(\mathrm{~d}, J_{\mathrm{C}-\mathrm{F}}=257.7 \mathrm{~Hz}\right), 138.7,135.2\left(\mathrm{~d}, J_{\mathrm{C}-\mathrm{F}}\right.$ $=5.1 \mathrm{~Hz}), 133.5,133.1\left(\mathrm{~d}, J_{\mathrm{C}-\mathrm{F}}=9.6 \mathrm{~Hz}\right), 131.2\left(\mathrm{~d}, J_{\mathrm{C}-\mathrm{F}}=15.1 \mathrm{~Hz}\right), 129.7,129.6,128.8,126.1(\mathrm{~d}$, $\left.J_{\mathrm{C}-\mathrm{F}}=3.0 \mathrm{~Hz}\right), 118.2,117.7\left(\mathrm{~d}, J_{\mathrm{C}-\mathrm{F}}=24.6 \mathrm{~Hz}\right), 109.0\left(\mathrm{~d}, J_{\mathrm{C}-\mathrm{F}}=4.0 \mathrm{~Hz}\right), 21.5$.

${ }^{19}$ F NMR (282 MHz, CDCl 3$): \delta[p p m]=-107.55--107.64(\mathrm{~m}, 1 \mathrm{~F})$.

HRMS (ESI-TOF) m/z: $[\mathrm{M}+\mathrm{H}]^{+}$Calcd for $\mathrm{C}_{14} \mathrm{H}_{11} \mathrm{FN} 212.0870$; Found 212.0869. 
21. 4-Fluoro-3-(trimethylsilyl)benzonitrile (3u)

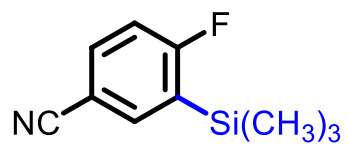

4-fluoro-3-(trimethylsilyl)benzonitrile was obtained from 1c via GMA using TMPMgCl-LiCl (1 M, 1.3 equiv., $0.65 \mathrm{~mL}$ ) and fresh distilled chlorotrimethylsilane (1.1 equiv., $0.55 \mathrm{mmol}, 70 \mu \mathrm{L}$ ). Purification via column chromatography (Hexanes/AcOEt 9:1) afforded the product as a yellow solid (0.31 mmol, $61 \mathrm{mg}, 63 \%)$.

Mp: $49-51{ }^{\circ} \mathrm{C}$.

${ }^{1}$ H-NMR (500 MHz, CDCl3): $\boldsymbol{\delta}[\mathbf{p p m}]=7.70(\mathrm{dd}, J=4.9,2.2 \mathrm{~Hz}, 1 \mathrm{H}), 7.68-7.64(\mathrm{~m}, 1 \mathrm{H})$, $7.08(\mathrm{t}, J=8.2 \mathrm{~Hz}, 1 \mathrm{H}), 0.34(\mathrm{~s}, 9 \mathrm{H})$.

${ }^{13} \mathbf{C}\left\{{ }^{1} \mathbf{H}\right\}$ NMR (126 MHz, CDCl 3$): \delta[\mathbf{p p m}]=169.5\left(\mathrm{~d}, J_{\mathrm{C}-\mathrm{F}}=250.9 \mathrm{~Hz}\right), 140.0\left(\mathrm{~d}, J_{\mathrm{C}-\mathrm{F}}=13.4\right.$ $\mathrm{Hz}), 135.6\left(\mathrm{~d}, J_{\mathrm{C}-\mathrm{F}}=10.0 \mathrm{~Hz}\right), 129.1\left(\mathrm{~d}, J_{\mathrm{C}-\mathrm{F}}=32.9 \mathrm{~Hz}\right), 118.6,116.1\left(\mathrm{~d}, J_{\mathrm{C}-\mathrm{F}}=27.9 \mathrm{~Hz}\right), 108.7(\mathrm{~d}$, $\left.J_{\mathrm{C}-\mathrm{F}}=3.4 \mathrm{~Hz}\right),-1.2(3 \mathrm{C})$.

${ }^{19}$ F NMR (282 MHz, CDCl 3$): \delta[p p m]=-90.55--90.61(\mathrm{~m}, 1 \mathrm{~F})$.

HRMS (ESI-TOF) m/z: $[\mathrm{M}+\mathrm{H}]^{+}$Calcd for $\mathrm{C}_{10} \mathrm{H}_{13} \mathrm{FNSi}$ 194.0796; Found 194.0793.

\section{3-Benzoyl-4-fluorobenzonitrile (3v)}

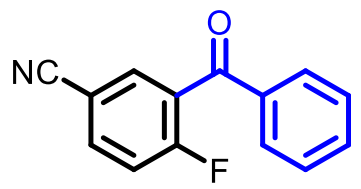

After applying GMA [TMPMgCl $\mathrm{LiCl}$ (1 M, 1.3 equiv., $0.65 \mathrm{~mL})$ ] for 1c followed by transmetalation with $\mathrm{ZnCl}_{2}(1 \mathrm{M}$ in THF, 1.3 equiv., $0.65 \mathrm{~mL})$, the benzoylation was performed using $\mathrm{Pd}\left(\mathrm{PPh}_{3}\right)_{4}(5 \mathrm{~mol} \%, 29 \mathrm{mg})$ and benzoyl chloride (1.0 equiv., $\left.0.5 \mathrm{mmol}, 58 \mu \mathrm{L}\right)$ at room temperature. Purification via column chromatography (Hexanes/AcOEt 9:1) afforded the product as a white solid (0.47 mmol, $107 \mathrm{mg}, 95 \%)$. Applying GMA at the same conditions followed by transmetalation with $\mathrm{CuCl} 2 \mathrm{LiCl}\left(0.5 \mathrm{M}\right.$ in $\mathrm{THF}, 1$ equiv.) at $-40{ }^{\circ} \mathrm{C}$ and the reaction of organocopper species with benzoyl chloride (1.0 equiv., $0.5 \mathrm{mmol}, 58 \mu \mathrm{L}$ ), furnished the same ketone in 78\%. CAS: 217498-80-1.

Mp: $92-95{ }^{\circ} \mathrm{C}$.

${ }^{1}$ H-NMR (400 MHz, CDCl3): $\boldsymbol{\delta}[\mathbf{p p m}]=7.87-7.78(\mathrm{~m}, 4 \mathrm{H}), 7,65(\mathrm{tt}, J=7.4,1.2 \mathrm{~Hz}, 1 \mathrm{H})$, $7.52-7.48(\mathrm{~m}, 2 \mathrm{H}), 7.31(\mathrm{t}, J=8.8 \mathrm{~Hz}, 1 \mathrm{H})$. 
${ }^{13} \mathbf{C}\left\{{ }^{1} \mathbf{H}\right\}$ NMR (101 MHz, $\left.\mathbf{C D C l}_{3}\right): \delta[\mathbf{p p m}]=190.8,162.1\left(\mathrm{~d}, J_{\mathrm{C}-\mathrm{F}}=261.3 \mathrm{~Hz}\right), 136.7\left(\mathrm{~d}, J_{\mathrm{C}-\mathrm{F}}\right.$ =9.5 Hz), 136.3, $135.1\left(\mathrm{~d}, J_{\mathrm{C}-\mathrm{F}}=4.4 \mathrm{~Hz}\right), 134.2,129.8(2 \mathrm{C}), 128.8(2 \mathrm{C}), 128.6\left(\mathrm{~d}, J_{\mathrm{C}-\mathrm{F}}=16.3 \mathrm{~Hz}\right)$, $118.0\left(\mathrm{~d}, J_{\mathrm{C}-\mathrm{F}}=23.4 \mathrm{~Hz}\right), 117.2,109.3\left(\mathrm{~d}, J_{\mathrm{C}-\mathrm{F}}=4.2 \mathrm{~Hz}\right)$.

${ }^{19}$ F NMR (282 MHz, CDCl 3$): \delta[p p m]=-101.35--101.42(\mathrm{~m}, 1 \mathrm{~F})$.

HRMS (ESI-TOF) m/z: [M+Na $]^{+}$Calcd for $\mathrm{C}_{14} \mathrm{H}_{8} \mathrm{FNNaO} 248.0482$; Found 248.0482.

The reported characterization data is in accordance with the literature. ${ }^{9}$

\section{6-Fluoro-[1,1'-biphenyl]-3-carbonitrile (3w)}

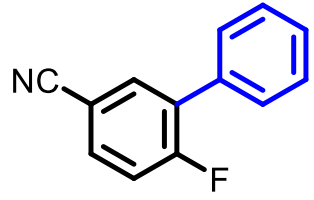

After applying GMA [TMPMgCl $\cdot \mathrm{LiCl}(1 \mathrm{M}, 1.3$ equiv., $3.7 \mathrm{~mL})$ and 4-fluorobenzonitrile (1c) (1.0 equiv., $2.84 \mathrm{mmol}, 344 \mathrm{mg}$ )] followed by transmetalation with $\mathrm{ZnCl}_{2}$ (1 M in THF, 1.3 equiv., $3.7 \mathrm{~mL})$, the Negishi cross-coupling was performed using $\mathrm{Pd}\left(\mathrm{PPh}_{3}\right)_{4}(5 \mathrm{~mol} \%, 164.1 \mathrm{mg})$ and iodobenzene (1.2 equiv., $3.41 \mathrm{mmol}, 0.38 \mathrm{~mL}$ ) at $50{ }^{\circ} \mathrm{C}$ (oil bath). Purification via column chromatography (Hexanes/AcOEt 9:1) afforded the product as a white solid (2.52 mmol, 497 mg, 89\%). CAS: 1214331-73-3.

Mp: $83-84{ }^{\circ} \mathrm{C}$.

${ }^{1}$ H-NMR (400 MHz, CDCl3): $\boldsymbol{\delta}[\mathbf{p p m}]=7.77(\mathrm{dd}, J=7.1,2.2 \mathrm{~Hz}, 1 \mathrm{H}), 7.64(\mathrm{ddd}, J=8.6,4.5$, $2.2 \mathrm{~Hz}, 1 \mathrm{H}), 7.53-7.42(\mathrm{~m}, 5 \mathrm{H}), 7.27$ (app t, $J=8.6 \mathrm{~Hz}, 1 \mathrm{H})$.

${ }^{13} \mathbf{C}\left\{{ }^{1} \mathbf{H}\right\}$ NMR (101 MHz, CDCl $): \delta[\mathbf{p p m}]=162.2\left(\mathrm{~d}, J_{\mathrm{C}-\mathrm{F}}=257.9 \mathrm{~Hz}\right), 135.2\left(\mathrm{~d}, J_{\mathrm{C}-\mathrm{F}}=5.2\right.$ $\mathrm{Hz}), 133.5,133.2\left(\mathrm{~d}, J_{\mathrm{C}-\mathrm{F}}=9.6 \mathrm{~Hz}\right), 131.1\left(\mathrm{~d}, J_{\mathrm{C}-\mathrm{F}}=15.2 \mathrm{~Hz}\right), 129.0\left(\mathrm{~d}, J_{\mathrm{C}-\mathrm{F}}=2.9 \mathrm{~Hz}, 2 \mathrm{C}\right), 128.9$ (2C), 128.9, 118.2, $117.7\left(\mathrm{~d}, J_{\mathrm{C}-\mathrm{F}}=24.6 \mathrm{~Hz}\right), 109.1\left(\mathrm{~d}, J_{\mathrm{C}-\mathrm{F}}=4.2 \mathrm{~Hz}\right)$.

${ }^{19}$ F NMR (282 MHz, CDCl 3$): \delta[p p m]=-107.71--107.79(\mathrm{~m}, 1 \mathrm{~F})$.

GC-MS (EI, 70 eV) m/z: 197.05 (100\%), 196.05 (28\%), 198.05 (18\%), 169.00 (14\%), 170.00 $(11 \%)$.

The reported characterization data is in accordance with the literature. ${ }^{10}$

${ }^{9}$ Ishihara, S.; Saito, F.; Mashiko, E.; Kono, K. Benzylamine analogs as ileac-type bile acid transporter inhibitors. Japan patent JP2000178188. 


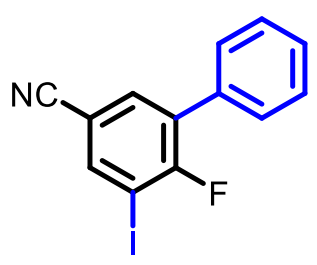

6-fluoro-5-iodo-[1,1'-biphenyl]-3-carbonitrile was obtained from $\mathbf{3 w}$ via GMA using TMPMgCl-LiCl (1 M, 1.3 equiv., $0.65 \mathrm{~mL}$ ), 6-fluoro-[1,1'-biphenyl]-3-carbonitrile (1.0 equiv., $0.5 \mathrm{mmol}, 99 \mathrm{mg}$ ) and iodine (1.1 equiv., $0.55 \mathrm{mmol}, 139.6 \mathrm{mg})$. Purification via column chromatography (Hexanes/AcOEt 9:1) afforded the product as a white solid (0.37 mmol, 119.5 mg, $74 \%)$.

Mp: $103-105^{\circ} \mathrm{C}$.

${ }^{1}$ H-NMR (400 MHz, CDCl3): $\boldsymbol{\delta}[\mathbf{p p m}]=8.03(\mathrm{dd}, J=5.1,2.1 \mathrm{~Hz}, 1 \mathrm{H}), 7.71(\mathrm{dd}, J=6.5,2.1$ $\mathrm{Hz}, 1 \mathrm{H}), 7.49-7.45(\mathrm{~m}, 5 \mathrm{H})$.

${ }^{13} \mathbf{C}\left\{{ }^{1} \mathbf{H}\right\}$ NMR (101 MHz, CDCl3): $\delta$ [ppm] $=161.3\left(\mathrm{~d}, J_{\mathrm{C}-\mathrm{F}}=255.6 \mathrm{~Hz}\right), 141.8\left(\mathrm{~d}, J_{\mathrm{C}-\mathrm{F}}=3.1\right.$ $\mathrm{Hz}), 135.1\left(\mathrm{~d}, J_{\mathrm{C}-\mathrm{F}}=4.5 \mathrm{~Hz}\right), 133.0\left(\mathrm{~d}, J_{\mathrm{C}-\mathrm{F}}=1.9 \mathrm{~Hz}\right), 131.2\left(\mathrm{~d}, J_{\mathrm{C}-\mathrm{F}}=17.8 \mathrm{~Hz}\right), 129.3,129.0$ (2C), $129.0(2 \mathrm{C}), 116.7,110.6\left(\mathrm{~d}, J_{\mathrm{C}-\mathrm{F}}=4.7 \mathrm{~Hz}\right), 83.4\left(\mathrm{~d}, J_{\mathrm{C}-\mathrm{F}}=29.3 \mathrm{~Hz}\right)$.

${ }^{19}$ F NMR (282 MHz, CDCl 3$): \delta[p p m]=-86.97(t, J=5.8 \mathrm{~Hz}, 1 \mathrm{~F})$.

GC-MS (EI, 70 eV) m/z: 322.95 (100\%), 195.00 (26\%), 169.00 (25\%), 323.95 (15\%), 195.95 $(14 \%)$.

HRMS (ESI-TOF) m/z: [M+CH$\left.{ }_{3} \mathrm{OH}+\mathrm{H}\right]^{+}$Calcd for $\mathrm{C}_{14} \mathrm{H}_{12} \mathrm{FINO} 355.9942$; Found 355.9950.

25. 6-Fluoro-5-(hydroxy(pyridin-3-yl)methyl)-[1,1'-biphenyl]-3-carbonitrile (4b)<smiles>N#Cc1cc(-c2ccccc2)c(F)c(C(O)c2cccnc2)c1</smiles>

6-fluoro-5-(hydroxy(pyridin-3-yl)methyl)-[1,1'-biphenyl]-3-carbonitrile was obtained from 3w via GMA using TMPMgCl-LiCl (1 M, 1.3 equiv., $0.65 \mathrm{~mL})$, 6-fluoro-[1,1'-biphenyl]-3-

${ }^{10}$ Danz, M.; Zink, D. Organic molecules for use in organic optoelectronic devices. German Patent WO 2017/005698 A1. 
carbonitrile (1.0 equiv., $0.5 \mathrm{mmol}, 99 \mathrm{mg}$ ) and 3-pyridinecarboxaldehyde (1.1 equiv., $0.55 \mathrm{mmol}$, $52 \mu \mathrm{L}$ ). Purification via column chromatography (Hexanes/AcOEt 7:3) afforded the product as a white solid (0.40 mmol, $121.6 \mathrm{mg}, 80 \%)$.

Mp: $127-130^{\circ} \mathrm{C}$.

${ }^{1}$ H-NMR (400 MHz, CDCl $): \delta[p p m]=8.58(\mathrm{~d}, J=1.5 \mathrm{~Hz}, 1 \mathrm{H}), 8.42(\mathrm{dd}, J=4.9,1.5 \mathrm{~Hz}$, $1 \mathrm{H}), 8.00(\mathrm{dd}, J=6.0,2.1 \mathrm{~Hz}, 1 \mathrm{H}), 7.82(\operatorname{app} \mathrm{dt}, J=8.0,1.9 \mathrm{~Hz}, 1 \mathrm{H}), 7.66(\mathrm{dd}, J=6.8,2.1 \mathrm{~Hz}$, $1 \mathrm{H}), 7.46-7.39(\mathrm{~m}, 5 \mathrm{H}), 7.35(\mathrm{dd}, J=8.0,4.9 \mathrm{~Hz}, 1 \mathrm{H}), 6.20(\mathrm{~s}, 1 \mathrm{H}), 5.03(\mathrm{~s}, 1 \mathrm{H})$.

${ }^{13} \mathbf{C}\left\{{ }^{1} \mathbf{H}\right\}$ NMR (101 MHz, CDCl 3$): \delta[\mathbf{p p m}]=158.8\left(\mathrm{~d}, J_{\mathrm{C}-\mathrm{F}}=257.4 \mathrm{~Hz}\right), 147.8,147.0\left(\mathrm{~d}, J_{\mathrm{C}-\mathrm{F}}=\right.$ $1.7 \mathrm{~Hz}), 139.1,135.9,134.4\left(\mathrm{~d}, J_{\mathrm{C}-\mathrm{F}}=5.1 \mathrm{~Hz}\right), 133.2\left(\mathrm{~d}, J_{\mathrm{C}-\mathrm{F}}=16.1 \mathrm{~Hz}\right), 133.1,131.0\left(\mathrm{~d}, J_{\mathrm{C}-\mathrm{F}}=\right.$ $15.5 \mathrm{~Hz}), 130.6\left(\mathrm{~d}, J_{\mathrm{C}-\mathrm{F}}=5.4 \mathrm{~Hz}\right), 129.0\left(\mathrm{~d}, J_{\mathrm{C}-\mathrm{F}}=4.4 \mathrm{~Hz}, 2 \mathrm{C}\right), 129.0,128.9(2 \mathrm{C}), 124.4,118.1$, $109.2\left(\mathrm{~d}, J_{\mathrm{C}-\mathrm{F}}=4.3 \mathrm{~Hz}\right), 67.2\left(\mathrm{~d}, J_{\mathrm{C}-\mathrm{F}}=3.8 \mathrm{~Hz}\right)$.

${ }^{19}$ F NMR (282 MHz, CDCl3): $\delta[p p m]=-113.41(\mathrm{t}, J=6.5 \mathrm{~Hz}, 1 \mathrm{~F})$.

HRMS (ESI-TOF) m/z: $[\mathrm{M}+\mathrm{H}]^{+}$Calcd for $\mathrm{C}_{19} \mathrm{H}_{14} \mathrm{FN}_{2} \mathrm{O} 305.1085$; Found 305.1070.

\section{2,4-Difluoro-3-iodobenzonitrile (7a)}

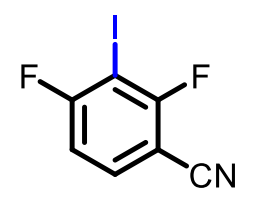

2,4-difluoro-3-iodobenzonitrile was obtained from 5a via GMB using TMPMgCl $\mathrm{LiCl}$ (1 M, 1.3 equiv., $0.65 \mathrm{~mL})$ at $0{ }^{\circ} \mathrm{C}$ or $(\mathrm{TMP})_{2} \mathrm{Zn} \cdot 2 \mathrm{MgCl}_{2} \cdot 2 \mathrm{LiCl}(0.29 \mathrm{M}$ in THF, 0.65 equiv., $1.1 \mathrm{~mL})$ at room temperature followed by trapping with iodine (1.1 equiv., $0.55 \mathrm{mmol}, 139.6 \mathrm{mg}$ ). Purification via column chromatography (Hexanes/AcOEt 9.7:0.3) afforded the product as a white solid (106 mg - 80\% ${ }^{11}$ and $\left.123.2 \mathrm{mg}-93 \%{ }^{12}\right)$. CAS: $1804885-55-9$.

Mp: $58-59^{\circ} \mathrm{C}$.

${ }^{1} \mathbf{H}-\mathbf{N M R}(400 \mathrm{MHz}, \mathbf{C D C l} 3): \boldsymbol{\delta}[\mathbf{p p m}]=7.65(\mathrm{ddd}, J=8.7,7.1,5.7 \mathrm{~Hz}, 1 \mathrm{H}), 7.02(\mathrm{ddd}, J=8.7$, $6.8,1.5 \mathrm{~Hz}, 1 \mathrm{H})$.

${ }^{13} \mathbf{C}\left\{{ }^{1} \mathbf{H}\right\}$ NMR (101 MHz, CDCl 3$): \delta[\mathbf{p p m}]=165.9\left(\mathrm{dd}, J_{\mathrm{C}-\mathrm{F}}=257.2,5.5 \mathrm{~Hz}\right), 164.0\left(\mathrm{dd}, J_{\mathrm{C}-\mathrm{F}}=\right.$ $258.2,6.7 \mathrm{~Hz}), 134.7\left(\mathrm{dd}, J_{\mathrm{C}-\mathrm{F}}=10.3,1.6 \mathrm{~Hz}\right), 112.7\left(\mathrm{dd}, J_{\mathrm{C}-\mathrm{F}}=25.6,3.8 \mathrm{~Hz}\right), 112.5\left(\mathrm{~d}, J_{\mathrm{C}-\mathrm{F}}=\right.$ $3.4 \mathrm{~Hz}), 98.3\left(\mathrm{dd}, J_{\mathrm{C}-\mathrm{F}}=18.2,4.0 \mathrm{~Hz}\right), 72.7\left(\mathrm{dd}, J_{\mathrm{C}-\mathrm{F}}=30.7,27.9 \mathrm{~Hz}\right)$.

\footnotetext{
${ }^{11}$ Metalation via TMPMgCl$\cdot \mathrm{LiCl}$

${ }^{12}$ Metalation via (TMP) ${ }_{2} \mathrm{Zn} \cdot 2 \mathrm{MgCl}_{2} \cdot 2 \mathrm{LiCl}$.
} 
${ }^{19}$ F NMR (282 MHz, CDCl $): \delta[p p m]=-79.96--80.03(\mathrm{~m}, 1 \mathrm{~F}),-82.90(\mathrm{dt}, J=6.9,1.4 \mathrm{~Hz}$, $1 \mathrm{~F})$.

GC-MS (EI, 70 eV) m/z: 264.90 (100\%), 138.05 (46\%), 88.00 (30\%), 265.90 (9\%), 87.00 (6\%).

Note: HRMS analysis of compound 7a was not possible due to low stability.

\section{3,4-Difluoro-5-iodobenzonitrile (7b)}

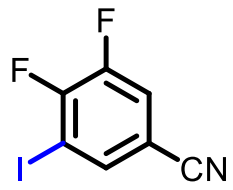

3,4-difluoro-5-iodobenzonitrile was obtained from 5c via GMB using TMPMgCl LiCl (1 M, 1.5 equiv., $0.75 \mathrm{~mL})$ at $-70{ }^{\circ} \mathrm{C}$ followed by transmetalation with $\mathrm{ZnCl}_{2}(1 \mathrm{M}$ in THF, 1.5 equiv., 0.75 $\mathrm{mL}$ ) during 20 minutes and quenching with iodine (1.1 equiv., $0.55 \mathrm{mmol}, 139.6 \mathrm{mg}$ ). Purification via column chromatography (Hexanes/AcOEt 9:1) afforded the product as a white solid (0.37 mmol, $99 \mathrm{mg}, 75 \%)$. CAS: 1439903-28-2.

Mp: $71-73^{\circ} \mathrm{C}$.

${ }^{1} \mathbf{H}-\mathbf{N M R}\left(400 \mathrm{MHz}, \mathbf{C D C l}_{3}\right): \delta[\mathbf{p p m}]=7.85(\mathrm{dt}, J=4.7,1.9 \mathrm{~Hz}, 1 \mathrm{H}), 7.49(\mathrm{ddd}, J=8.8,6.6$, $1.9 \mathrm{~Hz}, 1 \mathrm{H})$.

${ }^{13} \mathbf{C}\left\{{ }^{1} \mathbf{H}\right\}$ NMR (101 MHz, CDCl3): $\delta[\mathbf{p p m}]=154.0\left(\mathrm{dd}, J_{\mathrm{C}-\mathrm{F}}=256.2,13.4 \mathrm{~Hz}\right), 149.6\left(\mathrm{dd}, J_{\mathrm{C}-\mathrm{F}}\right.$ $=258.2,15.6 \mathrm{~Hz}), 138.4\left(\mathrm{dd}, J_{\mathrm{C}-\mathrm{F}}=4.0,1.4 \mathrm{~Hz}\right), 121.6\left(\mathrm{dd}, J_{\mathrm{C}-\mathrm{F}}=20.6,1.5 \mathrm{~Hz}\right), 115.6,110.7$ $\left(\mathrm{dd}, J_{\mathrm{C}-\mathrm{F}}=8.1,5.1 \mathrm{~Hz}\right), 83.7\left(\mathrm{~d}, J_{\mathrm{C}-\mathrm{F}}=23.9 \mathrm{~Hz}\right)$.

${ }^{19}$ F NMR (282 MHz, CDCl3): $\delta$ [ppm] = -106.06 - -106.18 (m, 1F), -129.90 - -130.02 (m, 1F). GC-MS (EI, 70 eV) m/z: 264.90 (100\%), 138.00 (56\%), 88.05 (31\%), 265.90 (9\%), 87.00 (8\%). Note: HRMS analysis of compound $\mathbf{7 b}$ was not possible due to low stability.

\section{3,5-Difluoro-4-iodobenzonitrile (7c)}

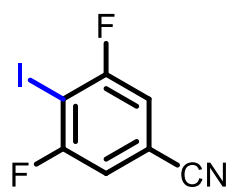

3,5-difluoro-4-iodobenzonitrile was obtained from $\mathbf{5 b}$ via $\mathbf{G M B}$ using TMPMgCl $\mathrm{LiCl}$ (1 M, 1.3 equiv., $0.65 \mathrm{~mL})$ at $-30{ }^{\circ} \mathrm{C}$ or $(\mathrm{TMP})_{2} \mathrm{Zn} \cdot 2 \mathrm{MgCl}_{2} \cdot 2 \mathrm{LiCl}(0.29 \mathrm{M}$ in THF, 0.65 equiv., $1.1 \mathrm{~mL})$ at room temperature followed by iodine (1.1 equiv., $0.55 \mathrm{mmol}, 139.6 \mathrm{mg})$. Purification via column 
chromatography (Hexanes/AcOEt 9:1) afforded the product as a white solid (93 $\mathrm{mg}-75 \%{ }^{13}$ and $\left.120.6 \mathrm{mg}-91 \%^{14}\right)$. CAS: $1487337-76-7$.

Mp: $127-130{ }^{\circ} \mathrm{C}$.

${ }^{1}$ H-NMR (400 MHz, CDCl3): $\delta$ [ppm] = $7.20-7.16(\mathrm{~m}, 2 \mathrm{H})$.

${ }^{13} \mathbf{C}\left\{{ }^{1} \mathbf{H}\right\}$ NMR (101 MHz, CDCl3): $\delta[\mathbf{p p m}]=163.0\left(\mathrm{dd}, J_{\mathrm{C}-\mathrm{F}}=250.6,6.3 \mathrm{~Hz}, 2 \mathrm{C}\right), 116.3\left(\mathrm{t}, J_{\mathrm{C}-\mathrm{F}}\right.$ $=3.4 \mathrm{~Hz}), 115.2-114.9(\mathrm{~m}, 2 \mathrm{C}), 114.4\left(\mathrm{t}, J_{\mathrm{C}-\mathrm{F}}=11.1 \mathrm{~Hz}\right), 79.1\left(\mathrm{t}, J_{\mathrm{C}-\mathrm{F}}=29.4 \mathrm{~Hz}\right)$.

${ }^{19}$ F NMR (282 MHz, CDCl 3$): \delta[p p m]=-87.41--87.47(\mathrm{~m}, 2 \mathrm{~F})$.

GC-MS (EI, 70 eV) m/z: 264.90 (100\%), 138.05 (43\%), 88.00 (31\%), 265.90 (9\%), 87.00 (7\%).

The reported characterization data is in accordance with the literature. ${ }^{15}$

\section{2,4-Difluoro-3-(hydroxy(2-methoxyphenyl)methyl)benzonitrile (7d)}<smiles>COc1ccccc1C(O)c1c(F)ccc(C#N)c1F</smiles>

2,4-difluoro-3-(hydroxy(2-methoxyphenyl)methyl)benzonitrile was obtained from 5a via GMB using TMPMgCl $\mathrm{LiCl}\left(1 \mathrm{M}, 1.3\right.$ equiv., $0.65 \mathrm{~mL}$ ) at $0{ }^{\circ} \mathrm{C}$ and 2-methoxybenzaldehyde (1.1 equiv., $0.55 \mathrm{mmol}, 75 \mathrm{mg}$ ). Purification via column chromatography (Hexanes/AcOEt 8:2) afforded the product as a white solid (0.47 mmol, $130.7 \mathrm{mg}, 95 \%)$.

Mp: $120-121^{\circ} \mathrm{C}$.

${ }^{1} \mathrm{H}-N M R\left(400 \mathrm{MHz}, \mathbf{C D C l}_{3}\right): \delta[\mathbf{p p m}]=7.55-7.48(\mathrm{~m}, 2 \mathrm{H}), 7.30(\mathrm{td}, J=8.1,1.7 \mathrm{~Hz}, 1 \mathrm{H})$, $7.01(\mathrm{td}, J=7.5,0.9 \mathrm{~Hz}, 1 \mathrm{H}), 6.97(\mathrm{td}, J=9.3,1.3 \mathrm{~Hz}, 1 \mathrm{H}), 6.86(\operatorname{app} \mathrm{dd}, J=8.1,0.7 \mathrm{~Hz}, 1 \mathrm{H})$, 6.38 (s, 1H), 3.79 (s, 3H), 2.78 (bs, 1H).

${ }^{13} \mathbf{C}\left\{{ }^{1} \mathbf{H}\right\}$ NMR (101 MHz, CDCl3): $\delta[\mathbf{p p m}]=164.0\left(\mathrm{dd}, J_{\mathrm{C}-\mathrm{F}}=260.7,8.5 \mathrm{~Hz}\right), 162.4\left(\mathrm{dd}, J_{\mathrm{C}-\mathrm{F}}=\right.$ 262.6, 9.1 Hz), 156.3, $133.3\left(\mathrm{dd}, J_{\mathrm{C}-\mathrm{F}}=11.6,2.3 \mathrm{~Hz}\right), 129.4,128.5,126.8\left(\mathrm{t}, J_{\mathrm{C}-\mathrm{F}}=2.0 \mathrm{~Hz}\right)$, $120.9\left(\mathrm{dd}, J_{\mathrm{C}-\mathrm{F}}=16.9,14.7 \mathrm{~Hz}\right), 120.7,113.6,113.3\left(\mathrm{dd}, J_{\mathrm{C}-\mathrm{F}}=24.4,3.8 \mathrm{~Hz}\right), 110.5,98.3\left(\mathrm{dd}, J_{\mathrm{C}-}\right.$ $\mathrm{F}=17.0,3.9 \mathrm{~Hz}), 64.3\left(\mathrm{t}, J_{\mathrm{C}-\mathrm{F}}=2.8 \mathrm{~Hz}\right), 55.4$.

\footnotetext{
${ }^{13}$ Metalation via TMPMgCl $\mathrm{LiCl}$.

${ }^{14}$ Metalation via (TMP) ${ }_{2} \mathrm{Zn} \cdot 2 \mathrm{MgCl}_{2} \cdot 2 \mathrm{LiCl}$.

${ }^{15}$ Huang, Y.; Cai, Z.; Li, S.; Nabulsi, N. Carson, R. Radiolabeled pharmaceuticals and methods of making and using same. U.S. Patent WO 2018/152339 A1.
} 
${ }^{19}$ F NMR (282 MHz, CDCl3): $\delta$ [ppm] = -101.26 - -101.35 (m, 1F), -104.96 (dd, $J=11.2,7.0$ $\mathrm{Hz}, 1 \mathrm{~F})$.

HRMS (ESI-TOF) m/z: [M+Na] $]^{+}$Calcd for $\mathrm{C}_{15} \mathrm{H}_{11} \mathrm{~F}_{2} \mathrm{NNaO}_{2}$ 298.0650; Found 298.0640.

30.4-((4-(Dimethylamino)phenyl)(hydroxy)methyl)-3,5-difluorobenzonitrilebenzonitrile (7e)<smiles>CN(C)c1ccc(C(O)c2c(F)cc(C#N)cc2F)cc1</smiles>

4-((4-(dimethylamino)phenyl)(hydroxy)methyl)-3,5-difluorobenzonitrilebenzonitrile was obtained from 5b via GMB using TMPMgCl $\mathrm{LiCl}(1 \mathrm{M}, 1.3$ equiv., $0.65 \mathrm{~mL})$ at $-30{ }^{\circ} \mathrm{C}$ and 4(dimethylamino)benzaldehyde (1.1 equiv., $0.55 \mathrm{mmol}, 82 \mathrm{mg}$ ) as the electrophile. Purification via column chromatography (Hexanes/AcOEt 8:2) afforded the product as a beige solid $(0.47$ mmol, $135.5 \mathrm{mg}, 94 \%)$.

Mp: $150-151{ }^{\circ} \mathrm{C}$.

${ }^{1}$ H-NMR (400 MHz, CDCl3): $\boldsymbol{\delta}$ [ppm] = $7.24(\operatorname{app~d}, J=8.4 \mathrm{~Hz}, 2 \mathrm{H}), 7.21(\operatorname{app~d}, J=7.7 \mathrm{~Hz}$, 2H), $6.71(\mathrm{~d}, J=8.4 \mathrm{~Hz}, 2 \mathrm{H}), 6.16(\mathrm{~s}, 1 \mathrm{H}), 2.95$ (s, 6H), 2.73 (bs, 1H).

${ }^{13} \mathbf{C}\left\{{ }^{1} \mathbf{H}\right\}$ NMR (101 MHz, CDCl 3$): \delta[\mathbf{p p m}]=160.6\left(\mathrm{dd}, J_{\mathrm{C}-\mathrm{F}}=252.4,9.1 \mathrm{~Hz}, 2 \mathrm{C}\right), 150.4,126.9$ $(2 \mathrm{C}), 126.1\left(\mathrm{t}, J_{\mathrm{C}-\mathrm{F}}=16.2 \mathrm{~Hz}\right), 116.6\left(\mathrm{t}, J_{\mathrm{C}-\mathrm{F}}=3.5 \mathrm{~Hz}\right), 116.3-116.0(\mathrm{~m}, 2 \mathrm{C}), 112.7\left(\mathrm{t}, J_{\mathrm{C}-\mathrm{F}}=\right.$ $12.4 \mathrm{~Hz}), 112.7$ (2C), 111.1, 68.0, 40.7 (2C).

${ }^{19}$ F NMR (282 MHz, CDCl3): $\delta[p p m]=-110.00--110.08(\mathrm{~m}, 2 \mathrm{~F})$.

HRMS (ESI-TOF) m/z: [M+H] ${ }^{+}$Calcd for $\mathrm{C}_{16} \mathrm{H}_{15} \mathrm{~F}_{2} \mathrm{~N}_{2} \mathrm{O} 289.1147$; Found 289.1135.

\section{3-(Benzo[d][1,3]dioxol-5-yl(hydroxy)methyl)-4,5-difluorobenzonitrile (7f)}<smiles>N#Cc1cc(F)c(F)c(C(O)c2ccc3c(c2)OCO3)c1</smiles>

3-(benzo[d][1,3]dioxol-5-yl(hydroxy)methyl)-4,5-difluorobenzonitrile was obtained from 5c via GMB using TMPMgCl $\operatorname{LiCl}(1 \mathrm{M}, 1.5$ equiv., $0.75 \mathrm{~mL})$ at $-70{ }^{\circ} \mathrm{C}$ and 1,3-benzodioxole-5carboxaldehyde (1.1 equiv., $0.55 \mathrm{mmol}, 82.6 \mathrm{mg}$ ) as the electrophile. Purification via column 
chromatography (Hexanes/AcOEt 8:2) afforded the product as a yellow oil (0.28 mmol, $82.4 \mathrm{mg}$, $57 \%)$.

${ }^{1}$ H-NMR (400 MHz, CDCl3): $\boldsymbol{\delta}[\mathbf{p p m}]=7.78-7.76(\mathrm{~m}, 1 \mathrm{H}), 7.39(\mathrm{ddd}, J=9.1,6.9,2.1 \mathrm{~Hz}$, $1 \mathrm{H}), 6.86-6.81(\mathrm{~m}, 2 \mathrm{H}), 6.78(\mathrm{~d}, J=7.9 \mathrm{~Hz}, 1 \mathrm{H}), 6.03(\mathrm{~s}, 1 \mathrm{H}), 5.96(\mathrm{~d}, J=1.4 \mathrm{~Hz}, 1 \mathrm{H}), 5.95(\mathrm{~d}$, $J=1.4 \mathrm{~Hz}, 1 \mathrm{H}), 2.31(\mathrm{bs}, 1 \mathrm{H})$.

${ }^{13} \mathbf{C}\left\{{ }^{1} \mathbf{H}\right\}$ NMR (101 MHz, CDCl3): $\delta[\mathbf{p p m}]=150.6\left(\mathrm{dd}, J_{\mathrm{C}-\mathrm{F}}=258.6,12.9 \mathrm{~Hz}\right), 150.2\left(\mathrm{dd}, J_{\mathrm{C}-\mathrm{F}}\right.$ $=253.4,13.5 \mathrm{~Hz}), 148.3,147.9,135.8\left(\mathrm{~d}, J_{\mathrm{C}-\mathrm{F}}=11.2 \mathrm{~Hz}\right), 135.2,127.0\left(\mathrm{t}, J_{\mathrm{C}-\mathrm{F}}=3.8 \mathrm{~Hz}\right), 120.2$ $\left(\mathrm{dd}, J_{\mathrm{C}-\mathrm{F}}=20.3,1.3 \mathrm{~Hz}\right), 120.2\left(\mathrm{~d}, J_{\mathrm{C}-\mathrm{F}}=0.9 \mathrm{~Hz}\right), 117.2,108.6,108.6\left(\mathrm{dd}, J_{\mathrm{C}-\mathrm{F}}=8.1,5.1 \mathrm{~Hz}\right)$ 106.9, 101.5, 69.3.

${ }^{19}$ F NMR (282 MHz, CDCl3): $\delta$ [ppm] $=-132.50(\mathrm{dt}, J=21.1,6.2 \mathrm{~Hz}, 1 \mathrm{~F}),-134.19--134.30$ (m, 1F).

GC-MS (EI, 70 eV) m/z: 289.05 (100\%), 123.05 (86\%), 93.05 (65\%), 151.00 (42\%), 165.95 $(31 \%)$.

HRMS (ESI-TOF) m/z: [M+FA-H] $]^{-}$Calcd for $\mathrm{C}_{16} \mathrm{H}_{10} \mathrm{~F}_{2} \mathrm{NO}_{5} 334.0533$; Found 334.0521.

\section{5,6-Difluoro-4'-nitro-[1,1'-biphenyl]-3-carbonitrile (7g)}

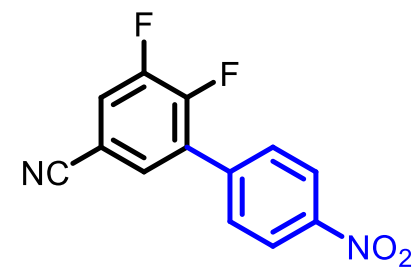

5,6-difluoro-4'-nitro-[1,1'-biphenyl]-3-carbonitrile was obtained from 5c via GMB using TMPMgCl LiCl (1 M, 1.5 equiv., $0.75 \mathrm{~mL})$ at $-70{ }^{\circ} \mathrm{C}$ followed by transmetalation with $\mathrm{ZnCl}_{2}(1$ $\mathrm{M}$ in THF, 1.5 equiv., $0.75 \mathrm{~mL}$ ) during 20 minutes and Negishi cross-coupling catalyzed by $\mathrm{Pd}\left(\mathrm{PPh}_{3}\right)_{4}(5 \mathrm{~mol} \%, 29 \mathrm{mg})$ in the presence of 1-iodo-4-nitrobenzene (1.2 equiv., $0.6 \mathrm{mmol}$, $149.4 \mathrm{mg}$ ) at $50{ }^{\circ} \mathrm{C}$ (oil bath). Purification via column chromatography (Hexanes/AcOEt 9:1) afforded the product as an orange solid $(0.29 \mathrm{mmol}, 75.5 \mathrm{mg}, 58 \%)$.

Mp: $162-164^{\circ} \mathrm{C}$.

${ }^{1}$ H-NMR (400 MHz, CDCl3): $\boldsymbol{\delta}[\mathbf{p p m}]=8.39-8.35(\mathrm{~m}, 2 \mathrm{H}), 7.73-7.70(\mathrm{~m}, 2 \mathrm{H}), 7.61-7.56$ (m, 2H). 
${ }^{13} \mathbf{C}\left\{{ }^{1} \mathbf{H}\right\}$ NMR (101 MHz, $\left.\mathbf{C D C l}_{3}\right): \delta[\mathbf{p p m}]=151.2\left(\mathrm{dd}, J_{\mathrm{C}-\mathrm{F}}=256.6,15.8 \mathrm{~Hz}\right), 151.0\left(\mathrm{dd}, J_{\mathrm{C}-\mathrm{F}}\right.$ $=264.0,15.8 \mathrm{~Hz}), 148.4,138.7,130.9\left(\mathrm{dd}, J_{\mathrm{C}-\mathrm{F}}=11.2,0.8 \mathrm{~Hz}\right), 130.1\left(\mathrm{~d}, J_{\mathrm{C}-\mathrm{F}}=3.1 \mathrm{~Hz}, 2 \mathrm{C}\right)$, $130.0\left(\mathrm{t}, J_{\mathrm{C}-\mathrm{F}}=3.0 \mathrm{~Hz}\right), 124.3(2 \mathrm{C}), 121.4\left(\mathrm{dd}, J_{\mathrm{C}-\mathrm{F}}=20.0,2.4 \mathrm{~Hz}\right), 116.6,109.4\left(\mathrm{dd}, J_{\mathrm{C}-\mathrm{F}}=8.5\right.$, $5.4 \mathrm{~Hz}$ ).

GC-MS (EI, 70 eV) m/z: 260.00 (100\%), 202.00 (46\%), 213.00 (44\%), 214.00 (38\%), 187.00 (33\%).

${ }^{19}$ F NMR (282 MHz, CDCl3): $\delta$ [ppm] = -131.74 - -131.85 (m, 1F), -131.93 - -132.05 (m, 1F).

HRMS (ESI-TOF) m/z: [M+CH$\left.{ }_{3} \mathrm{OH}+\mathrm{H}\right]^{+}$Calcd for $\mathrm{C}_{14} \mathrm{H}_{11} \mathrm{~F}_{2} \mathrm{~N}_{2} \mathrm{O}_{3}$ 293.0732; Found 293.0741.

\section{4-Benzoyl-3,5-difluorobenzonitrile $(7 \mathrm{~h})$}

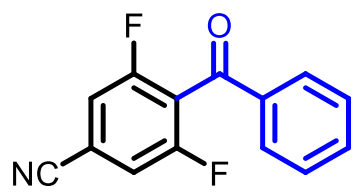

4-benzoyl-3,5-difluorobenzonitrile was obtained from $\mathbf{5 b}$ via $\mathbf{G M B}$ using TMPMgCl LiCl (1 M, 1.3 equiv., $0.65 \mathrm{~mL}$ ) at $-30{ }^{\circ} \mathrm{C}$ followed by transmetalation with $\mathrm{ZnCl}_{2}$ (1 M in THF, 1.3 equiv., $0.65 \mathrm{~mL})$ for 20 minutes and palladium catalyzed benzoylation using $\mathrm{Pd}\left(\mathrm{PPh}_{3}\right)_{4}(5 \mathrm{~mol} \%, 29 \mathrm{mg})$ and benzoyl chloride (1.0 equiv., $0.5 \mathrm{mmol}, 58 \mu \mathrm{L}$ ) at room temperature. Purification via column chromatography (Hexanes/AcOEt 9:1) afforded the product as a white solid (0.41 mmol, 100.9 $\mathrm{mg}, 83 \%)$.

Mp: $104-105^{\circ} \mathrm{C}$.

${ }^{1}$ H-NMR (400 MHz, CDCl3): $\boldsymbol{\delta}[\mathbf{p p m}]=7.83(\operatorname{app} \mathrm{d}, J=7.7 \mathrm{~Hz}, 2 \mathrm{H}), 7.68(\mathrm{tt}, J=7.1,1.3 \mathrm{~Hz}$, $1 \mathrm{H}), 7.55-7.50(\mathrm{~m}, 2 \mathrm{H}), 7.37-7.32(\mathrm{~m}, 2 \mathrm{H})$.

${ }^{13} \mathbf{C}\left\{{ }^{1} \mathbf{H}\right\}$ NMR (101 MHz, CDCl3): $\boldsymbol{\delta}[\mathbf{p p m}]=186.9,159.7\left(\mathrm{dd}, J_{\mathrm{C}-\mathrm{F}}=255.5,8.5 \mathrm{~Hz}, 2 \mathrm{C}\right)$, 135.9, 135.2, $129.7(2 \mathrm{C}), 129.2(2 \mathrm{C}), 122.1\left(\mathrm{t}, J_{\mathrm{C}-\mathrm{F}}=22.7 \mathrm{~Hz}\right), 116.4-116.1(\mathrm{~m}, 2 \mathrm{C}), 116.1(\mathrm{t}$, $\left.J_{\mathrm{C}-\mathrm{F}}=3.5 \mathrm{~Hz}\right), 115.5\left(\mathrm{t}, J_{\mathrm{C}-\mathrm{F}}=11.4 \mathrm{~Hz}\right)$.

${ }^{19}$ F NMR (282 MHz, CDCl 3$): \delta[p p m]=-107.68--107.73(\mathrm{~m}, 2 \mathrm{~F})$.

GC-MS (EI, 70 eV) m/z: 105.05 (100\%), 243.00 (74\%), 77.00 (54\%), 165.95 (30\%), 51.00 $(18 \%)$.

HRMS (ESI-TOF) m/z: $\left[\mathrm{M}+\mathrm{CH}_{3} \mathrm{OH}+\mathrm{H}\right]^{+}$Calcd for $\mathrm{C}_{15} \mathrm{H}_{12} \mathrm{~F}_{2} \mathrm{NO}_{2} 276.0831$; Found 276.0839. 


\section{2,6-Difluoro-4'-methoxy-[1,1'-biphenyl]-4-carbonitrile (7i)}<smiles>COc1ccc(-c2c(F)cc(C#N)cc2F)cc1</smiles>

2,6-difluoro-4'-methoxy-[1,1'-biphenyl]-4-carbonitrile was obtained from $\mathbf{5 b}$ via $\mathbf{G M B}$ using TMPMgCl $\mathrm{LiCl}(1 \mathrm{M}, 1.3$ equiv., $0.65 \mathrm{~mL})$ at $-30{ }^{\circ} \mathrm{C}$ followed by transmetalation with $\mathrm{ZnCl}_{2}(1$ $\mathrm{M}$ in THF, 1.3 equiv., $0.65 \mathrm{~mL})$ and Negishi cross-coupling catalyzed by $\mathrm{Pd}\left(\mathrm{PPh}_{3}\right)_{4}(5 \mathrm{~mol} \%, 29$ $\mathrm{mg}$ ) in the presence of 4-methoxyiodobenzene (1.2 equiv., $0.6 \mathrm{mmol}, 140.4 \mathrm{mg}$ ) at $50{ }^{\circ} \mathrm{C}$ (oil bath). Purification via column chromatography (Hexanes/AcOEt 9:1) afforded the product as a white solid (0.44 mmol, $107.9 \mathrm{mg}, 88 \%)$. CAS: 2222566-95-0.

Mp: $150-151^{\circ} \mathrm{C}$.

${ }^{1}$ H-NMR (400 MHz, CDCl3): $\boldsymbol{\delta}[\mathbf{p p m}]=7.44-7.39(\mathrm{~m}, 2 \mathrm{H}), 7.33-7.26(\mathrm{~m}, 2 \mathrm{H}), 7.03-7.00$ (m, 2H), $3.87(\mathrm{~s}, 3 \mathrm{H})$.

${ }^{13} \mathbf{C}\left\{{ }^{1} \mathbf{H}\right\}$ NMR (101 MHz, CDCl 3$): \delta[\mathbf{p p m}]=160.5,160.0\left(\mathrm{dd}, J_{\mathrm{C}-\mathrm{F}}=251.8,8.0 \mathrm{~Hz}, 2 \mathrm{C}\right), 131.6$ $\left(\mathrm{t}, J_{\mathrm{C}-\mathrm{F}}=2.3 \mathrm{~Hz}, 2 \mathrm{C}\right), 124.1\left(\mathrm{t}, J_{\mathrm{C}-\mathrm{F}}=18.3 \mathrm{~Hz}\right), 119.3,116.9\left(\mathrm{t}, J_{\mathrm{C}-\mathrm{F}}=3.4 \mathrm{~Hz}\right), 116.2-115.9(\mathrm{~m}$, 2C), $114.2(2 \mathrm{C}), 111.7\left(\mathrm{t}, J_{\mathrm{C}-\mathrm{F}}=12.3 \mathrm{~Hz}\right), 55.5$.

${ }^{19}$ F NMR (282 MHz, CDCl 3$): \delta[p p m] ~=-110.59--110.66(\mathrm{~m}, 2 \mathrm{~F})$.

GC-MS (EI, 70 eV) m/z: 245.05 (100\%), 202.00 (59\%), 230.00 (25\%), 175.95 (19\%), 246.05 $(17 \%)$.

The reported characterization data is in accordance with the literature. ${ }^{16}$

35. 4-(7-Chloroquinolin-4-yl)-3,5-difluorobenzonitrile (7j)<smiles>N#Cc1cc(F)c(-c2ccnc3cc(Cl)ccc23)c(F)c1</smiles>

4-(7-chloroquinolin-4-yl)-3,5-difluorobenzonitrile was obtained from $\mathbf{5 b}$ via $\mathbf{G M B}$ using TMPMgCl $\mathrm{LiCl}(1 \mathrm{M}, 1.3$ equiv., $0.65 \mathrm{~mL})$ at $-30{ }^{\circ} \mathrm{C}$ followed by transmetalation with $\mathrm{ZnCl}_{2}(1$ $\mathrm{M}$ in THF, 1.3 equiv., $0.65 \mathrm{~mL})$ and Negishi cross-coupling catalyzed by $\mathrm{Pd}\left(\mathrm{PPh}_{3}\right)_{4}(5 \mathrm{~mol} \%, 29$

${ }^{16}$ Luo, Z.-J.; Zhao, H.-Y.; Zhang, X. Highly Selective Pd-Catalyzed Direct C-F Bond Arylation of Polyfluoroarenes. Org. Lett. 2018, 20 (9), 2543-2546. 
$\mathrm{mg}$ ) in the presence of 7-chloro-4-iodoquinoline (1.2 equiv., $0.6 \mathrm{mmol}, 173.7 \mathrm{mg}$ ) at $50{ }^{\circ} \mathrm{C}$ (oil bath). Purification via column chromatography (Hexanes/AcOEt 8:2) afforded the product as a white solid (0.24 mmol, $72.2 \mathrm{mg}, 48 \%)$.

Mp: $142-144^{\circ} \mathrm{C}$.

${ }^{1}$ H-NMR (400 MHz, CDCl 3$): \delta[p p m]=9.05(d, J=4.5 \mathrm{~Hz}, 1 \mathrm{H}), 8.27(\mathrm{~d}, J=2.1 \mathrm{~Hz}, 1 \mathrm{H}), 7.53$ $(\mathrm{dd}, J=8.9,2.1 \mathrm{~Hz}, 1 \mathrm{H}), 7.47-7.42(\mathrm{~m}, 3 \mathrm{H}), 7.41(\mathrm{~d}, J=4.5 \mathrm{~Hz}, 1 \mathrm{H})$.

${ }^{13} \mathbf{C}\left\{{ }^{1} \mathbf{H}\right\}$ NMR (101 MHz, CDCl3): $\boldsymbol{\delta}[\mathbf{p p m}]=160.1\left(\mathrm{dd}, J_{\mathrm{C}-\mathrm{F}}=254.6,7.2 \mathrm{~Hz}, 2 \mathrm{C}\right), 150.4$, $148.3,136.6,134.8,129.1,128.8,126.1,124.7,123.0,119.6\left(t, J_{\mathrm{C}-\mathrm{F}}=20.3 \mathrm{~Hz}\right), 116.4-116.1$ $(\mathrm{m}, 2 \mathrm{C}), 116.2\left(\mathrm{t}, J_{\mathrm{C}-\mathrm{F}}=3.4 \mathrm{~Hz}\right), 115.0\left(\mathrm{t}, J_{\mathrm{C}-\mathrm{F}}=11.7 \mathrm{~Hz}\right)$.

${ }^{19}$ F NMR (282 MHz, CDCl3): $\delta$ [ppm] = -106.24--106.30 (m, 2F).

HRMS (ESI-TOF) m/z: [M+H] ${ }^{+}$Calcd for $\mathrm{C}_{16} \mathrm{H}_{8} \mathrm{ClF}_{2} \mathrm{~N}_{2}$ 301.0339; Found 301.0352.

\section{3,4-Difluoro-2-iodobenzonitrile (7k)}

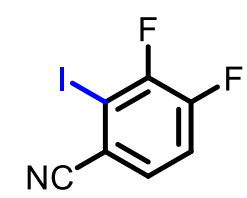

3,4-difluoro-2-iodobenzonitrile was obtained from $\mathbf{5 c}$ via GMB using (TMP) ${ }_{2} \mathrm{Zn} \cdot 2 \mathrm{MgCl}_{2} \cdot 2 \mathrm{LiCl}$ (0.29 $\mathrm{M}$ in THF, 0.75 equiv., $1.3 \mathrm{~mL})$ at room temperature and iodine (1.1 equiv., $0.55 \mathrm{mmol}$, 139.6 mg). Purification via column chromatography (Hexanes/AcOEt 9.5:0.5) afforded the product as a white solid (0.38 mmol, $101 \mathrm{mg}, 76 \%)$. CAS: 1803847-48-4.

Mp: $67-69^{\circ} \mathrm{C}$.

1H-NMR (400 MHz, DMSO-d $): \delta[\mathbf{p p m}]=7.40(\mathrm{ddd}, J=8.8,4.6,1.8 \mathrm{~Hz}, 1 \mathrm{H}), 7.29(\mathrm{td}, J=$ 8.8, 7.2 Hz, 1H).

${ }^{13} \mathbf{C}\left\{{ }^{1} \mathbf{H}\right\}$ NMR (101 MHz, DMSO-d $): \delta[\mathbf{p p m}]=152.4\left(\mathrm{dd}, J_{\mathrm{C}-\mathrm{F}}=263.6,15.1 \mathrm{~Hz}\right), 151.6(\mathrm{dd}$, $\left.J_{\mathrm{C}-\mathrm{F}}=250.0,14.0 \mathrm{~Hz}\right), 131.0\left(\mathrm{dd}, J_{\mathrm{C}-\mathrm{F}}=7.6,4.3 \mathrm{~Hz}\right), 118.6\left(\mathrm{dd}, J_{\mathrm{C}-\mathrm{F}}=18.9,0.9 \mathrm{~Hz}\right), 117.9$, $117.8\left(\mathrm{dd}, J_{\mathrm{C}-\mathrm{F}}=4.1,1.3 \mathrm{~Hz}\right), 89.1\left(\mathrm{~d}, J_{\mathrm{C}-\mathrm{F}}=25.3 \mathrm{~Hz}\right)$.

${ }^{19}$ F NMR (282 MHz, CDCl3): $\delta$ [ppm] = -109.65 (ddd, $\left.J=23.0,7.2,1.7 \mathrm{~Hz}, 1 \mathrm{~F}\right),-123.99(\mathrm{ddd}$, $J=23.0,9.0,4.6 \mathrm{~Hz}, 1 \mathrm{~F})$.

GC-MS (EI, 70 eV) m/z: 264.90 (100\%), 138.05 (50\%), 88.05 (33\%), 265.90 (9\%), 87.00 (7\%). 
Note: HRMS analysis of compound 7k was not possible due to low stability.

\section{5,6-Difluoro-[1,1'-biphenyl]-2,4' -dicarbonitrile (7l)}

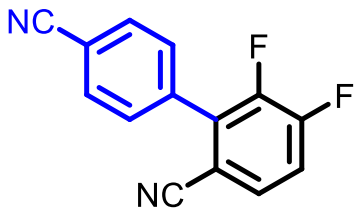

5,6-difluoro-[1,1'-biphenyl]-2,4'-dicarbonitrile was obtained from 5c via GMB using (TMP) ${ }_{2} \mathrm{Zn} \cdot 2 \mathrm{MgCl}_{2} \cdot 2 \mathrm{LiCl}(0.29 \mathrm{M}$ in $\mathrm{THF}, 0.75$ equiv., $1.3 \mathrm{~mL})$ at room temperature and subsequent Negishi cross-coupling catalyzed by $\mathrm{Pd}\left(\mathrm{PPh}_{3}\right)_{4}(5 \mathrm{~mol} \%, 29 \mathrm{mg})$ in the presence of 4iodobenzonitrile (1.2 equiv., $0.6 \mathrm{mmol}, 137.4 \mathrm{mg}$ ) at $50{ }^{\circ} \mathrm{C}$ (oil bath). Purification via column chromatography (Hexanes/AcOEt 8:2) afforded the product as a brown solid (0.31 mmol, 74.5 $\mathrm{mg}, 62 \%)$.

Mp: $160-162{ }^{\circ} \mathrm{C}$.

${ }^{1}$ H-NMR (400 MHz, DMSO-d6): $\boldsymbol{\delta}$ [ppm] = 7.84 (app. d, $J=8.6$ Hz, 2H), $7.64-7.60$ (m, 3H), $7.37(\mathrm{td}, J=8.9,7.3 \mathrm{~Hz}, 1 \mathrm{H})$.

${ }^{13} \mathbf{C}\left\{{ }^{1} \mathbf{H}\right\}$ NMR (101 MHz, DMSO-d $): \delta[\mathbf{p p m}]=153.9\left(\mathrm{dd}, J_{\mathrm{C}-\mathrm{F}}=260.8,13.4 \mathrm{~Hz}\right), 148.1(\mathrm{dd}$, $\left.J_{\mathrm{C}-\mathrm{F}}=253.3,13.7 \mathrm{~Hz}\right), 135.0\left(\mathrm{~d}, J_{\mathrm{C}-\mathrm{F}}=2.5 \mathrm{~Hz}\right), 133.8\left(\mathrm{dd}, J_{\mathrm{C}-\mathrm{F}}=14.2,1.1 \mathrm{~Hz}\right), 132.7,130.7(\mathrm{~d}$, $\left.J_{\mathrm{C}-\mathrm{F}}=1.9 \mathrm{~Hz}\right), 130.6\left(\mathrm{dd}, J_{\mathrm{C}-\mathrm{F}}=8.0,4.8 \mathrm{~Hz}\right), 118.5\left(\mathrm{~d}, J_{\mathrm{C}-\mathrm{F}}=18.6 \mathrm{~Hz}\right), 118.2,116.3\left(\mathrm{app} . \mathrm{dd}, J_{\mathrm{C}-}\right.$ $\mathrm{F}=3.3,1.9 \mathrm{~Hz}), 113.9,109.2\left(\right.$ app. dd, $\left.J_{\mathrm{C}-\mathrm{F}}=3.7,2.7 \mathrm{~Hz}\right)$.

${ }^{19}$ F NMR (282 MHz, CDCl 3$): \delta[p p m]=-125.49(\mathrm{ddd}, J=21.7,9.1,4.6 \mathrm{~Hz}, 1 \mathrm{~F}),-136.50--$ $136.61(\mathrm{~m}, 1 \mathrm{~F})$.

HRMS (ESI-TOF) m/z: [M+Na] $]^{+}$Calcd for $\mathrm{C}_{14} \mathrm{H}_{7} \mathrm{~F}_{2} \mathrm{~N}_{2} \mathrm{Na}$ 263.0391; Found 263.0389.

\section{3-(2-Chlorobenzoyl)-2,4-difluorobenzonitrile (7m)}

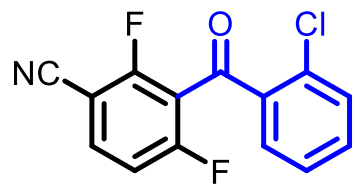

3-(2-chlorobenzoyl)-2,4-difluorobenzonitrile was obtained from 5a via GMB using $(\mathrm{TMP})_{2} \mathrm{Zn} 2 \mathrm{MgCl}_{2} \cdot 2 \mathrm{LiCl}(0.29 \mathrm{M}$ in $\mathrm{THF}, 0.65$ equiv., $1.1 \mathrm{~mL})$ at room temperature and subsequent palladium catalyzed benzoylation using $\mathrm{Pd}\left(\mathrm{PPh}_{3}\right)_{4}(5 \mathrm{~mol} \%, 29 \mathrm{mg})$ and 2chlorobenzoyl chloride (1.0 equiv., $0.5 \mathrm{mmol}, 63 \mu \mathrm{L})$ at the same temperature. Purification via 
column chromatography (Hexanes/AcOEt 9:1) afforded the product as a white solid (0.30 mmol, $83.3 \mathrm{mg}, 60 \%$ ).

Mp: $69-72^{\circ} \mathrm{C}$.

${ }^{1}$ H-NMR (400 MHz, DMSO-d $): \delta[p p m]=7.77(\mathrm{ddd}, J=8.7,7.0,5.7 \mathrm{~Hz}, 1 \mathrm{H}), 7.68(\mathrm{dd}, J=$ 7.7, $1.7 \mathrm{~Hz}, 1 \mathrm{H}), 7.53(\mathrm{ddd}, J=8.2,7.3,1.7 \mathrm{~Hz}, 1 \mathrm{H}), 7.45(\mathrm{dd}, J=8.2,1.1 \mathrm{~Hz}, 1 \mathrm{H}), 7.42(\mathrm{td}, J=$ 7.7, $1.3 \mathrm{~Hz}, 1 \mathrm{H}), 7.12(\mathrm{td}, J=8.7,1.4 \mathrm{~Hz}, 1 \mathrm{H})$.

${ }^{13} \mathrm{C}\left\{{ }^{1} \mathbf{H}\right\}$ NMR (101 MHz, DMSO-d $): \boldsymbol{\delta}[\mathbf{p p m}]=185.9,162.9\left(\mathrm{dd}, J_{\mathrm{C}-\mathrm{F}}=264.6,6.7 \mathrm{~Hz}\right), 161.5$ $\left(\mathrm{dd}, J_{\mathrm{C}-\mathrm{F}}=266.4,7.7 \mathrm{~Hz}\right), 136.7,136.5\left(\mathrm{dd}, J_{\mathrm{C}-\mathrm{F}}=11.1,1.9 \mathrm{~Hz}\right), 134.2,132.9,131.3,131.1$, $127.5,119.7\left(\mathrm{dd}, J_{\mathrm{C}-\mathrm{F}}=19.8,17.5 \mathrm{~Hz}\right), 113.8\left(\mathrm{dd}, J_{\mathrm{C}-\mathrm{F}}=23.2,4.0 \mathrm{~Hz}\right), 112.6\left(\mathrm{~d}, J_{\mathrm{C}-\mathrm{F}}=1.2 \mathrm{~Hz}\right)$, $99.0\left(\mathrm{dd}, J_{\mathrm{C}-\mathrm{F}}=16.2,4.2 \mathrm{~Hz}\right)$.

${ }^{19}$ F NMR (282 MHz, CDCl3): $\delta$ [ppm] = -100.10 (td, $\left.J=8.5,5.7 \mathrm{~Hz}, 1 \mathrm{~F}\right),-103.68--103.74$ $(\mathrm{m}, 1 \mathrm{~F})$.

HRMS (ESI-TOF) m/z: [M+Na] $]^{+}$Calcd for $\mathrm{C}_{14} \mathrm{H}_{6} \mathrm{ClF}_{2} \mathrm{NNaO} 299.9998$; Found 300.0022 .

\section{2,4',6-Trifluoro-[1,1'-biphenyl]-4-carbonitrile (7n)}

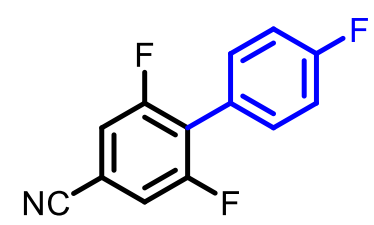

2,4',6-trifluoro-[1,1'-biphenyl]-4-carbonitrile was obtained from $\mathbf{5 b}$ via $\mathbf{G M B}$ using $(\mathrm{TMP})_{2} \mathrm{Zn} \cdot 2 \mathrm{MgCl}_{2} \cdot 2 \mathrm{LiCl}(0.29 \mathrm{M}$ in $\mathrm{THF}, 0.65$ equiv., $1.1 \mathrm{~mL})$ at room temperature and subsequent Negishi cross-coupling catalyzed by $\mathrm{Pd}\left(\mathrm{PPh}_{3}\right)_{4}(5 \mathrm{~mol} \%, 29 \mathrm{mg})$ in the presence of 4fluoroiodobenzene (1.2 equiv., $0.6 \mathrm{mmol}, 69 \mu \mathrm{L}$ ) at $50^{\circ} \mathrm{C}$ (oil bath). Purification via column chromatography (Hexanes/AcOEt 9.5:0.5) afforded the product as a white solid (0.46 mmol, $107.3 \mathrm{mg}, 92 \%)$.

Mp: $195-196{ }^{\circ} \mathrm{C}$.

${ }^{1}$ H-NMR (400 MHz, DMSO-d6): $\delta$ [ppm] = 7.47 - 7.44 (m, 2H), $7.35-7.29$ (m, 2H), 7.22 $7.17(\mathrm{~m}, 2 \mathrm{H})$.

${ }^{13} \mathbf{C}\left\{{ }^{1} \mathbf{H}\right\}$ NMR (101 MHz, DMSO-d $): \delta[\mathbf{p p m}]=163.4\left(\mathrm{~d}, J_{\mathrm{C}-\mathrm{F}}=250.1 \mathrm{~Hz}\right), 160.0\left(\mathrm{dd}, J_{\mathrm{C}-\mathrm{F}}=\right.$ 252.5, 7.7 Hz, 2C), $132.2\left(\mathrm{dt}, J_{\mathrm{C}-\mathrm{F}}=8.5,2.1 \mathrm{~Hz}, 2 \mathrm{C}\right), 123.3\left(\mathrm{t}, J_{\mathrm{C}-\mathrm{F}}=18.3 \mathrm{~Hz}\right), 123.1\left(\mathrm{~d}, J_{\mathrm{C}-\mathrm{F}}=\right.$ 
$3.4 \mathrm{~Hz}), 116.7\left(\mathrm{t}, J_{\mathrm{C}-\mathrm{F}}=3.5 \mathrm{~Hz}\right), 116.3-115.9(\mathrm{~m}, 2 \mathrm{C}), 116.0\left(\mathrm{~d}, J_{\mathrm{C}-\mathrm{F}}=21.9 \mathrm{~Hz}, 2 \mathrm{C}\right), 112.6(\mathrm{t}$, $\left.J_{\mathrm{C}-\mathrm{F}}=12.3 \mathrm{~Hz}\right)$.

${ }^{19}$ F NMR (282 MHz, CDCl3): $\delta$ [ppm] = -110.32 - -110.39 (m, 2F), -110.98 - -111.07 (m, 1F).

GC-MS (EI, 70 eV) m/z: 233.00 (100\%), 234.05 (18\%), 213.00 (14\%), 232.05 (10\%), 116.55 $(9 \%)$.

HRMS (ESI-TOF) m/z: [M+CH$\left.{ }_{3} \mathrm{OH}+\mathrm{H}\right]^{+}$Calcd for $\mathrm{C}_{14} \mathrm{H}_{11} \mathrm{~F}_{3} \mathrm{NO} 266.0787$; Found 266.0797.

\section{5-Fluoro-4-iodopicolinonitrile (10a)}

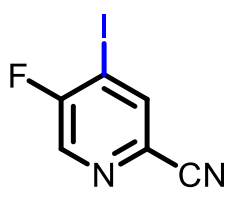

5-fluoro-4-iodopicolinonitrile was obtained from 8a via GMC using TMPMgCl-LiCl (1 M, 1.3 equiv., $0.65 \mathrm{~mL}$ ) at $-30^{\circ} \mathrm{C}$ and iodine (1.1 equiv., $\left.0.55 \mathrm{mmol}, 139.6 \mathrm{mg}\right)$. Purification via column chromatography (Hexanes/AcOEt 8:2) afforded the product as a white solid $(0.35 \mathrm{mmol}, 86.8$ mg, 70\%). CAS: 1807159-50-7.

Mp: $130-133{ }^{\circ} \mathrm{C}$.

${ }^{1} \mathbf{H}-\mathbf{N M R}\left(400 \mathrm{MHz}, \mathbf{C D C l}_{3}\right): \boldsymbol{\delta}[\mathbf{p p m}]=8.41(\mathrm{~s}, 1 \mathrm{H}), 8.14(\mathrm{~d}, J=4.6 \mathrm{~Hz}, 1 \mathrm{H})$.

${ }^{13} \mathbf{C}\left\{{ }^{1} \mathbf{H}\right\}$ NMR (101 MHz, CDCl$): \delta[p p m]=161.4\left(\mathrm{~d}, J_{\mathrm{C}-\mathrm{F}}=264.2 \mathrm{~Hz}\right), 139.3\left(\mathrm{~d}, J_{\mathrm{C}-\mathrm{F}}=5.7\right.$ $\mathrm{Hz}), 139.1\left(\mathrm{~d}, J_{\mathrm{C}-\mathrm{F}}=34.1 \mathrm{~Hz}\right), 130.4\left(\mathrm{~d}, J_{\mathrm{C}-\mathrm{F}}=5.5 \mathrm{~Hz}\right), 115.2,93.1\left(\mathrm{~d}, J_{\mathrm{C}-\mathrm{F}}=24.8 \mathrm{~Hz}\right)$.

${ }^{19}$ F NMR (282 MHz, CDCl3): $\delta[p p m]=-99.10(\mathrm{~d}, J=4.6 \mathrm{~Hz}, 1 \mathrm{~F})$.

GC-MS (EI, 70 eV) m/z: 247.95 (100\%), 121.00 (51\%), 94.00 (24\%), 69.00 (12\%), 101.00 $(8 \%)$.

HRMS (ESI-TOF) m/z: [M+H] $]^{+}$Calcd for $\mathrm{C}_{6} \mathrm{H}_{3} \mathrm{FIN}_{2} 248.9320$; Found 248.9324.

\section{2-Fluoro-3-iodoisonicotinonitrile (10b)}<smiles>N#Cc1ccnc(F)c1I</smiles>

2-fluoro-3-iodoisonicotinonitrile was obtained from 8c via GMC using TMPMgCl-LiCl (1 M, 1.5 equiv., $0.75 \mathrm{~mL}$ ) at $-70{ }^{\circ} \mathrm{C}$ followed by transmetalation with $\mathrm{ZnCl}_{2}(1 \mathrm{M}$ in THF, 1.5 equiv., 
$0.75 \mathrm{~mL})$ or $(\mathrm{TMP})_{2} \mathrm{Zn} \cdot 2 \mathrm{MgCl}_{2} \cdot 2 \mathrm{LiCl}(0.29 \mathrm{M}$ in THF, 0.9 equiv., $1.5 \mathrm{~mL})$ at room temperature with iodine (1.1 equiv., $0.55 \mathrm{mmol}, 139.6 \mathrm{mg}$ ) as the electrophile. Purification via column chromatography (Hexanes/AcOEt 9:1) afforded the product as a white solid (85.6 $\mathrm{mg}-69 \%{ }^{17}$ and $\left.94.2 \mathrm{mg}-76 \%^{18}\right)$. CAS: $898854-64-3$.

Mp: $96-97^{\circ} \mathrm{C}$.

${ }^{1}$ H-NMR (400 MHz, CDCl 3$): \delta[p p m]=8.33(\mathrm{dd}, J=5.0,0.8 \mathrm{~Hz}, 1 \mathrm{H}), 7.40(\mathrm{dd}, J=5.0,1.0$ $\mathrm{Hz}, 1 \mathrm{H})$.

${ }^{13} \mathrm{C}\left\{{ }^{1} \mathrm{H}\right\}$ NMR (101 MHz, CDCl$): \delta[\mathbf{p p m}]=163.1\left(\mathrm{~d}, J_{\mathrm{C}-\mathrm{F}}=237.6 \mathrm{~Hz}\right), 148.3\left(\mathrm{~d}, J_{\mathrm{C}-\mathrm{F}}=14.4\right.$ $\mathrm{Hz}), 133.2\left(\mathrm{~d}, J_{\mathrm{C}-\mathrm{F}}=4.2 \mathrm{~Hz}\right), 125.2\left(\mathrm{~d}, J_{\mathrm{C}-\mathrm{F}}=5.5 \mathrm{~Hz}\right), 116.6\left(\mathrm{~d}, J_{\mathrm{C}-\mathrm{F}}=4.9 \mathrm{~Hz}\right), 81.8\left(\mathrm{~d}, J_{\mathrm{C}-\mathrm{F}}=\right.$ $47.1 \mathrm{~Hz})$.

${ }^{19}$ F NMR (282 MHz, CDCl 3$): \delta[p p m] ~=-48.43(s, 1 F)$.

GC-MS (EI, 70 eV) m/z: 247.90 (100\%), 121.05 (76\%), 76.00 (23\%), 94.00 (13\%), 101.00 $(9 \%)$.

Note: HRMS analysis of compound 10b was not possible due to low stability.

\section{2-Fluoro-4-iodonicotinonitrile (10c)}<smiles>N#Cc1c(I)ccnc1F</smiles>

2-fluoro-4-iodonicotinonitrile was obtained from 8b via GMC using TMPMgCl LiCl (1 M, 1.5 equiv., $0.75 \mathrm{~mL}$ ) at $-70{ }^{\circ} \mathrm{C}$ and iodine (1.1 equiv., $0.55 \mathrm{mmol}, 139.6 \mathrm{mg}$ ) as the electrophile. Purification via column chromatography (Hexanes/AcOEt 8.5:1.5) afforded the product as a white solid (0.42 mmol, $105.4 \mathrm{mg}, 85 \%)$. CAS: 898854-59-6.

Mp: $85-86^{\circ} \mathrm{C}$.

${ }^{1} \mathbf{H}-\mathbf{N M R}(400 \mathrm{MHz}, \mathbf{C D C l} 3): \boldsymbol{\delta}[\mathbf{p p m}]=8.08(\mathrm{dd}, J=5.3,0.9 \mathrm{~Hz}, 1 \mathrm{H}), 7.80(\mathrm{dd}, J=5.3,0.9$ $\mathrm{Hz}, 1 \mathrm{H})$.

${ }^{13} \mathbf{C}\left\{{ }^{1} \mathbf{H}\right\}$ NMR (101 MHz, CDCl $): \delta[\mathbf{p p m}]=163.0\left(\mathrm{~d}, J_{\mathrm{C}-\mathrm{F}}=251.1 \mathrm{~Hz}\right), 150.9\left(\mathrm{~d}, J_{\mathrm{C}-\mathrm{F}}=15.8\right.$ $\mathrm{Hz}), 132.2\left(\mathrm{~d}, J_{\mathrm{C}-\mathrm{F}}=4.8 \mathrm{~Hz}\right), 114.0,113.7\left(\mathrm{~d}, J_{\mathrm{C}-\mathrm{F}}=6.1 \mathrm{~Hz}\right), 106.4\left(\mathrm{~d}, J_{\mathrm{C}-\mathrm{F}}=32.7 \mathrm{~Hz}\right)$.

\footnotetext{
${ }^{17}$ Metalation via TMPMgCl$\cdot \mathrm{LiCl}$.

${ }^{18}$ Metalation via (TMP) ${ }_{2} \mathrm{Zn} \cdot 2 \mathrm{MgCl}_{2} \cdot 2 \mathrm{LiCl}$.
} 
${ }^{19}$ F NMR (282 MHz, CDCl 3$): \delta[p p m]=-57.27(s, 1 F)$.

GC-MS (EI, 70 eV) m/z: 247.95 (100\%), 121.05 (77\%), 76.00 (24\%), 94.00 (15\%), 75.00 $(15 \%)$.

Note: HRMS analysis of compound 10c was not possible due to low stability.

\section{4-(4-Chlorophenyl)-5-fluoropicolinonitrile (10d)}

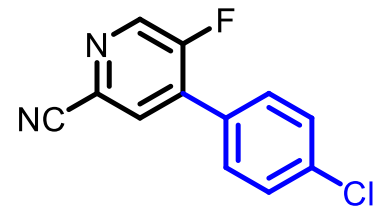

4-(4-chlorophenyl)-5-fluoropicolinonitrile was obtained from 8a via GMC using TMPMgCl $\mathrm{LiCl}(1 \mathrm{M}, 1.3$ equiv., $0.65 \mathrm{~mL})$ at $-30{ }^{\circ} \mathrm{C}$ followed by transmetalation with $\mathrm{ZnCl}_{2}(1$ $\mathrm{M}$ in THF, 1.3 equiv., $0.65 \mathrm{~mL})$ and a Negishi cross-coupling catalyzed by $\mathrm{Pd}\left(\mathrm{PPh}_{3}\right)_{4}(5 \mathrm{~mol} \%$, $29 \mathrm{mg}$ ) in the presence of 1-chloro-4-iodobenzene (1.2 equiv., $0.6 \mathrm{mmol}, 143.1 \mathrm{mg}$ ) at $50{ }^{\circ} \mathrm{C}$ (oil bath). Purification via column chromatography (Hexanes/AcOEt 9:1) afforded the product as a white solid (0.28 mmol, $65.1 \mathrm{mg}, 56 \%)$.

Mp: $130-131{ }^{\circ} \mathrm{C}$

${ }^{1}$ H-NMR (400 MHz, CDCl3): $\boldsymbol{\delta}[\mathbf{p p m}]=8.63(\mathrm{~d}, J=2.2 \mathrm{~Hz}, 1 \mathrm{H}), 7.82(\mathrm{~d}, J=6.0 \mathrm{~Hz}, 1 \mathrm{H}), 7.57$ $-7.50(\mathrm{~m}, 4 \mathrm{H})$.

${ }^{13} \mathbf{C}\left\{{ }^{1} \mathbf{H}\right\}$ NMR (101 MHz, CDCl 3$): \delta[\mathbf{p p m}]=157.7\left(\mathrm{~d}, J_{\mathrm{C}-\mathrm{F}}=266.8 \mathrm{~Hz}\right), 141.3\left(\mathrm{~d}, J_{\mathrm{C}-\mathrm{F}}=28.0\right.$ $\mathrm{Hz}), 136.9,136.4\left(\mathrm{~d}, J_{\mathrm{C}-\mathrm{F}}=11.7 \mathrm{~Hz}\right), 130.5\left(\mathrm{~d}, J_{\mathrm{C}-\mathrm{F}}=5.4 \mathrm{~Hz}\right), 130.2\left(\mathrm{~d}, J_{\mathrm{C}-\mathrm{F}}=3.7 \mathrm{~Hz}, 2 \mathrm{C}\right), 129.7$ $(2 \mathrm{C}), 129.5\left(\mathrm{~d}, J_{\mathrm{C}-\mathrm{F}}=2.7 \mathrm{~Hz}\right), 129.3\left(\mathrm{~d}, J_{\mathrm{C}-\mathrm{F}}=1.8 \mathrm{~Hz}\right), 116.6$.

${ }^{19}$ F NMR (282 MHz, CDCl 3$): \delta[p p m]=-123.46--123.49(\mathrm{~m}, 1 \mathrm{~F})$.

HRMS (ESI-TOF) m/z: [M+H $]^{+}$Calcd for $\mathrm{C}_{12} \mathrm{H}_{7} \mathrm{ClFN}_{2} 233.0277$; Found 233.0292.

44. 4-((5-Bromothiophen-2-yl)(hydroxy)methyl)-5-fluoropicolinonitrile (10e)<smiles>N#Cc1cc(C(O)c2ccc(Br)s2)c(F)cn1</smiles>

4-((5-bromothiophen-2-yl)(hydroxy)methyl)-5-fluoropicolinonitrile was obtained from 8a via GMC using TMPMgCl $\mathrm{LiCl}(1 \mathrm{M}, 1.3$ equiv., $0.65 \mathrm{~mL})$ at $-30{ }^{\circ} \mathrm{C}$ and 5-bromo-2thiophenecarboxaldehyde (1.1 equiv., $0.55 \mathrm{mmol}, 65 \mu \mathrm{L})$ as the electrophile. Purification via 
column chromatography (Hexanes/AcOEt 8:2) afforded the product as a white solid (0.31 mmol, $97.1 \mathrm{mg}, 62 \%)$.

Mp: $113-115^{\circ} \mathrm{C}$.

${ }^{1}$ H-NMR (400 MHz, CDCl 3$): \delta[p p m]=8.48(\mathrm{~d}, J=1.1 \mathrm{~Hz}, 1 \mathrm{H}), 8.08(\mathrm{~d}, J=5.6 \mathrm{~Hz}, 1 \mathrm{H}), 6.92$ $(\mathrm{d}, J=3.8 \mathrm{~Hz}, 1 \mathrm{H}), 6.77(\mathrm{dt}, J=3.8,0.7 \mathrm{~Hz}, 1 \mathrm{H}), 6.28(\mathrm{~d}, J=0.7 \mathrm{~Hz}, 1 \mathrm{H}), 2.53(\mathrm{bs}, 1 \mathrm{H})$.

${ }^{13} \mathbf{C}\left\{{ }^{1} \mathbf{H}\right\}$ NMR (101 MHz, CDCl3): $\delta[\mathbf{p p m}]=157.5\left(\mathrm{~d}, J_{\mathrm{C}-\mathrm{F}}=266.0 \mathrm{~Hz}\right), 145.2,140.2\left(\mathrm{~d}, J_{\mathrm{C}-\mathrm{F}}=\right.$ $25.9 \mathrm{~Hz}), 140.0\left(\mathrm{~d}, J_{\mathrm{C}-\mathrm{F}}=12.9 \mathrm{~Hz}\right), 130.4\left(\mathrm{~d}, J_{\mathrm{C}-\mathrm{F}}=5.2 \mathrm{~Hz}\right), 130.0,127.0\left(\mathrm{~d}, J_{\mathrm{C}-\mathrm{F}}=2.8 \mathrm{~Hz}\right)$, $126.2\left(\mathrm{~d}, J_{\mathrm{C}-\mathrm{F}}=1.2 \mathrm{~Hz}\right), 116.6,114.0,64.9\left(\mathrm{~d}, J_{\mathrm{C}-\mathrm{F}}=2.5 \mathrm{~Hz}\right)$.

${ }^{19}$ F NMR (282 MHz, CDCl3): $\delta[\mathbf{p p m}]=-122.56(\mathrm{~s}, 1 \mathrm{~F})$.

GC-MS (EI, 70 eV) m/z: 232.95 (100\%), 84.00 (24\%), 313.85 (21\%), 311.90 (21\%), 149.00 $(19 \%)$.

HRMS (ESI-TOF) m/z: [M-H] $]^{-}$Calcd for $\mathrm{C}_{11} \mathrm{H}_{5} \mathrm{BrFN}_{2} \mathrm{OS} 310.9295$; Found 310.9293.

\section{2-Fluoro-4-(4-methoxyphenyl)nicotinonitrile (10f)}

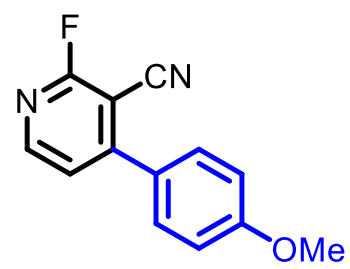

2-fluoro-4-(4-methoxyphenyl)nicotinonitrile was obtained from $\mathbf{8 b}$ via $\mathbf{G M C}$ using TMPMgCl $\mathrm{LiCl}(1 \mathrm{M}, 1.5$ equiv., $0.75 \mathrm{~mL})$ at $-70{ }^{\circ} \mathrm{C}$ followed by transmetalation with $\mathrm{ZnCl}_{2}(1$ $\mathrm{M}$ in THF, 1.5 equiv., $0.75 \mathrm{~mL})$ and a Negishi cross-coupling reaction catalyzed by $\mathrm{Pd}\left(\mathrm{PPh}_{3}\right)_{4}(5$ $\mathrm{mol} \%, 29 \mathrm{mg}$ ) in the presence of 4-methoxyiodobenzene (1.2 equiv., $0.6 \mathrm{mmol}, 140.4 \mathrm{mg}$ ) at 50 ${ }^{\circ} \mathrm{C}$ (oil bath). Purification via column chromatography (Hexanes/AcOEt 8:2) afforded the product as a brown solid (0.26 mmol, $59.3 \mathrm{mg}, 52 \%)$.

Mp: $136-139{ }^{\circ} \mathrm{C}$.

${ }^{1} \mathrm{H}-\mathrm{NMR}(400 \mathrm{MHz}, \mathbf{C D C l} 3): \boldsymbol{\delta}[\mathbf{p p m}]=8.38(\mathrm{~d}, J=5.3 \mathrm{~Hz}, 1 \mathrm{H}), 7.65-7.61$ (m, 2H), 7.36 (dd, $J=5.3,0.9 \mathrm{~Hz}, 1 \mathrm{H}), 7.08-7.04(\mathrm{~m}, 2 \mathrm{H}), 3.89(\mathrm{~s}, 3 \mathrm{H})$.

${ }^{13} \mathbf{C}\left\{{ }^{1} \mathbf{H}\right\}$ NMR (101 MHz, CDCl 3$): \delta[\mathbf{p p m}]=164.4\left(\mathrm{~d}, J_{\mathrm{C}-\mathrm{F}}=246.1 \mathrm{~Hz}\right), 161.9,157.6,150.8$ $\left(\mathrm{d}, J_{\mathrm{C}-\mathrm{F}}=16.1 \mathrm{~Hz}\right), 130.2(2 \mathrm{C}), 126.8\left(\mathrm{~d}, J_{\mathrm{C}-\mathrm{F}}=3.1 \mathrm{~Hz}\right), 121.7\left(\mathrm{~d}, J_{\mathrm{C}-\mathrm{F}}=4.2 \mathrm{~Hz}\right), 114.9(2 \mathrm{C})$, $113.1\left(\mathrm{~d}, J_{\mathrm{C}-\mathrm{F}}=4.5 \mathrm{~Hz}\right), 95.0\left(\mathrm{~d}, J_{\mathrm{C}-\mathrm{F}}=31.0 \mathrm{~Hz}\right), 55.6$. 
${ }^{19}$ F NMR (282 MHz, CDCl 3$): \delta[p p m]=-60.42(\mathrm{~s}, 1 \mathrm{~F})$.

HRMS (ESI-TOF) m/z: [M+Na $]^{+}$Calcd for $\mathrm{C}_{13} \mathrm{H}_{9} \mathrm{FN}_{2} \mathrm{NaO} 251.0591$; Found 251.0584.

\section{2-Fluoro-3-phenylisonicotinonitrile (10g)}

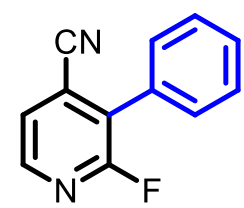

2-fluoro-3-phenylisonicotinonitrile was obtained from 8c via GMC using $(\mathrm{TMP})_{2} \mathrm{Zn} 2 \mathrm{MgCl}_{2} \cdot 2 \mathrm{LiCl}(0.29 \mathrm{M}$ in THF, 0.9 equiv., $1.5 \mathrm{~mL})$ at room temperature and subsequent Negishi cross-coupling catalyzed by $\mathrm{Pd}\left(\mathrm{PPh}_{3}\right)_{4}(5 \mathrm{~mol} \%, 29 \mathrm{mg})$ in the presence of iodobenzene (1.2 equiv., $0.6 \mathrm{mmol}, 67 \mu \mathrm{L}$ ) at $50{ }^{\circ} \mathrm{C}$ (oil bath). Purification via column chromatography (Hexanes/AcOEt 9.5:0.5) afforded the product as a beige solid (0.36 mmol, 71.4 mg, $72 \%)$.

Mp: $57-58{ }^{\circ} \mathrm{C}$.

${ }^{1}$ H-NMR (400 MHz, CDCl3): $\boldsymbol{\delta}[\mathbf{p p m}]=8.35(\mathrm{dd}, J=5.1,1.0 \mathrm{~Hz}, 1 \mathrm{H}), 7.56(\mathrm{dd}, J=5.1,0.8$ $\mathrm{Hz}, 1 \mathrm{H}), 7.54-7.53(\mathrm{~m}, 5 \mathrm{H})$.

${ }^{13} \mathbf{C}\left\{{ }^{1} \mathbf{H}\right\}$ NMR (101 MHz, CDCl3): $\delta[\mathbf{p p m}]=160.7\left(\mathrm{~d}, J_{\mathrm{C}-\mathrm{F}}=241.7 \mathrm{~Hz}\right), 147.2\left(\mathrm{~d}, J_{\mathrm{C}-\mathrm{F}}=15.5\right.$ $\mathrm{Hz}), 130.2,129.8\left(\mathrm{~d}, J_{\mathrm{C}-\mathrm{F}}=4.6 \mathrm{~Hz}\right), 129.7\left(\mathrm{~d}, J_{\mathrm{C}-\mathrm{F}}=1.7 \mathrm{~Hz}, 2 \mathrm{C}\right), 129.0(2 \mathrm{C}), 127.4\left(\mathrm{~d}, J_{\mathrm{C}-\mathrm{F}}=\right.$ $33.6 \mathrm{~Hz}), 124.7\left(\mathrm{~d}, J_{\mathrm{C}-\mathrm{F}}=5.3 \mathrm{~Hz}\right), 124.1\left(\mathrm{~d}, J_{\mathrm{C}-\mathrm{F}}=5.6 \mathrm{~Hz}\right), 115.4\left(\mathrm{~d}, J_{\mathrm{C}-\mathrm{F}}=5.7 \mathrm{~Hz}\right)$.

${ }^{19}$ F NMR (282 MHz, CDCl 3$): \delta[p p m]=-66.01(\mathrm{~s}, 1 \mathrm{~F})$.

GC-MS (EI, 70 eV) m/z: 198.00 (100\%), 197.00 (29\%), 199.05 (16\%), 171.00 (15\%), 151.00 $(13 \%)$.

HRMS (ESI-TOF) m/z: [M+CH$\left.{ }_{3} \mathrm{OH}+\mathrm{H}\right]^{+}$Calcd for $\mathrm{C}_{13} \mathrm{H}_{12} \mathrm{FN}_{2} \mathrm{O}$ 231.0928; Found 231.0934.

\section{2-Fluoro-3-(m-tolyl)isonicotinonitrile (10h)}

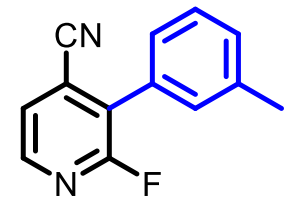

2-fluoro-3-(m-tolyl)isonicotinonitrile was obtained from 8c via GMC using $(\mathrm{TMP})_{2} \mathrm{Zn} 2 \mathrm{MgCl}_{2} \cdot 2 \mathrm{LiCl}(0.29 \mathrm{M}$ in $\mathrm{THF}, 0.9$ equiv., $1.5 \mathrm{~mL})$ at room temperature and 
subsequent Negishi cross-coupling catalyzed by $\mathrm{Pd}\left(\mathrm{PPh}_{3}\right)_{4}(5 \mathrm{~mol} \%, 29 \mathrm{mg})$ in the presence of 3 iodotoluene (1.2 equiv., $0.6 \mathrm{mmol}, 77 \mu \mathrm{L}$ ) at $50{ }^{\circ} \mathrm{C}$ (oil bath). Purification via column chromatography (Hexanes/AcOEt 9.5:0.5) afforded the product as a colorless oil (0.40 mmol, $84.9 \mathrm{mg}, 80 \%)$.

${ }^{1}$ H-NMR (400 MHz, CDCl3): $\delta[\mathbf{p p m}]=8.34(\mathrm{dd}, J=5.1,1.0 \mathrm{~Hz}, 1 \mathrm{H}), 7.55(\mathrm{dd}, J=5.1,0.8$ $\mathrm{Hz}, 1 \mathrm{H}), 7.44-7.40(\mathrm{~m}, 1 \mathrm{H}), 7.33-7.32(\mathrm{~m}, 3 \mathrm{H}), 2.44$ (s, 3H).

${ }^{13} \mathbf{C}\left\{{ }^{1} \mathbf{H}\right\}$ NMR (101 MHz, CDCl 3$): \delta[\mathbf{p p m}]=160.7\left(\mathrm{~d}, J_{\mathrm{C}-\mathrm{F}}=241.7 \mathrm{~Hz}\right), 147.1\left(\mathrm{~d}, J_{\mathrm{C}-\mathrm{F}}=15.4\right.$ $\mathrm{Hz}), 138.8,130.9,130.2\left(\mathrm{~d}, J_{\mathrm{C}-\mathrm{F}}=1.7 \mathrm{~Hz}\right), 129.7\left(\mathrm{~d}, J_{\mathrm{C}-\mathrm{F}}=3.9 \mathrm{~Hz}\right), 128.9,127.5\left(\mathrm{~d}, J_{\mathrm{C}-\mathrm{F}}=33.8\right.$ $\mathrm{Hz}), 126.8\left(\mathrm{~d}, J_{\mathrm{C}-\mathrm{F}}=1.7 \mathrm{~Hz}\right), 124.6\left(\mathrm{~d}, J_{\mathrm{C}-\mathrm{F}}=5.3 \mathrm{~Hz}\right), 124.1\left(\mathrm{~d}, J_{\mathrm{C}-\mathrm{F}}=5.7 \mathrm{~Hz}\right), 115.5\left(\mathrm{~d}, J_{\mathrm{C}-\mathrm{F}}=\right.$ $5.6 \mathrm{~Hz}), 21.5$.

${ }^{19}$ F NMR (282 MHz, CDCl 3$): \delta[p p m]=-65.90(\mathrm{~s}, 1 \mathrm{~F})$.

GC-MS (EI, 70 eV) m/z: 212.05 (100\%), 211.05 (83\%), 184.00 (30\%), 185.00 (19\%), 165.00 $(14 \%)$.

HRMS (ESI-TOF) m/z: [M+CH$\left.{ }_{3} \mathrm{OH}+\mathrm{H}\right]^{+}$Calcd for $\mathrm{C}_{14} \mathrm{H}_{14} \mathrm{FN}_{2} \mathrm{O}$ 245.1085; Found 245.1092.

\section{EXAMPLES OF SYNTHETIC APPLICATIONS OF THE FUNCTIONALIZED NITRILES}

Synthesis of 3-phenyl-1H-indazole-5-carbonitrile (11)

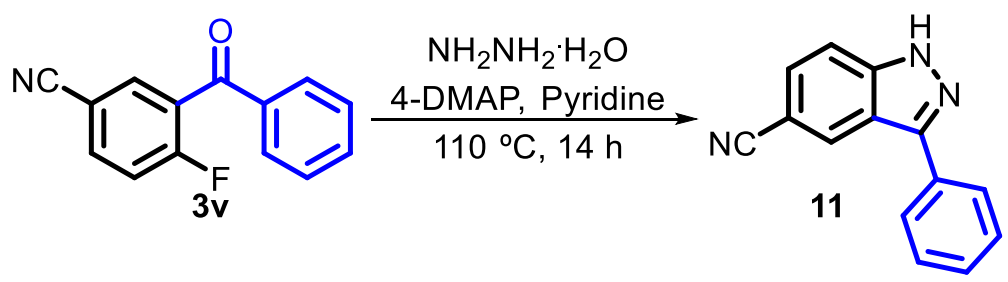

3-phenyl-1H-indazole-5-carbonitrile was prepared according to a modified procedure from Zhu and coworkers. ${ }^{19}$ A mixture of 3-benzoyl-4-fluorobenzonitrile (112.6 mg, $0.5 \mathrm{mmol}, 1$ equiv.), 4DMAP (61.1 mg, $0.5 \mathrm{mmol}, 1$ equiv.), and hydrazine monohydrate (0.24 mL, $5 \mathrm{mmol}, 10$ equiv.) in pyridine $(3.0 \mathrm{~mL})$ was kept at $110{ }^{\circ} \mathrm{C}$ (oil bath) for $14 \mathrm{~h}$. Then, water was added $(10 \mathrm{~mL})$, and the reaction was extracted with ethyl acetate $(3 \times 10 \mathrm{~mL})$. After drying with $\mathrm{MgSO}_{4}$, filtration, and concentration under reduced pressure, it was purified by column chromatography (silica gel, hexanes/ethyl acetate 7:3) as a colorless oil (91\%, $100 \mathrm{mg})$. CAS: 83684-54-2.

19 Lei, N.-P.; Fu, Y.-H.; Zhu, X.-Q. Elemental Step Thermodynamics of Various Analogues of Indazolium Alkaloids to Obtaining Hydride in Acetonitrile. Org. Biomol. Chem. 2015, 13 (47), 11472-11485. 


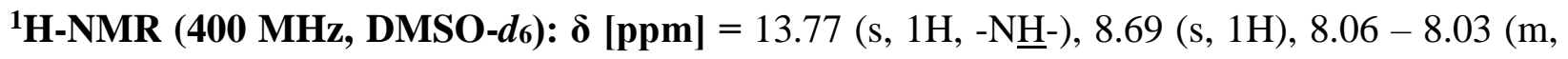
2H), $7.77(\mathrm{~d}, J=8.7,0.8 \mathrm{~Hz}, 1 \mathrm{H}), 7.73(\mathrm{~d}, J=8.7,1.3 \mathrm{~Hz}, 1 \mathrm{H}), 7.56-7.52(\mathrm{~m}, 2 \mathrm{H}), 7.47-7.43$ $(\mathrm{m}, 1 \mathrm{H})$.

${ }^{13} \mathbf{C}\left\{{ }^{1} \mathbf{H}\right\}$ NMR (101 MHz, DMSO-d $): \delta$ [ppm] = 144.6, 142.4, 132.4, 129.1 (2C), 128.4, 128.3, $127.8,127.1(2 \mathrm{C}), 119.8,119.7,112.2,103.5$.

GC-MS (EI, 70 eV) m/z: 219.05 (100\%), 77.00 (26\%), 218.05 (22\%), 220.05 (18\%), 192.00 $(15 \%)$.

HRMS (ESI-TOF) m/z: [M-H] Calcd for $\mathrm{C}_{14} \mathrm{H}_{8} \mathrm{~N}_{3}$ 218.0724; Found 218.0704.

\section{Synthesis of 4-(4-methoxyphenyl)dibenzo $[b, f][1,4]$ oxazepin-11-amine (12)}

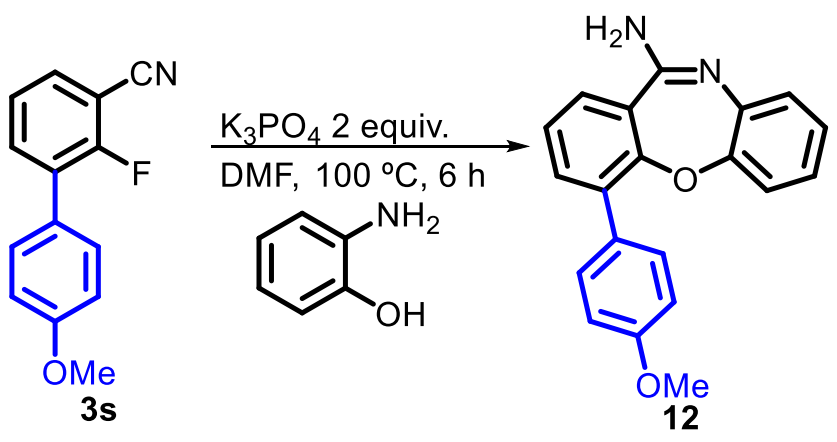

4-(4-methoxyphenyl)dibenzo[ $b, f][1,4]$ oxazepin-11-amine was prepared according to the procedure of $\mathrm{Wu}$ and Feng. ${ }^{20} \mathrm{~A} 5-\mathrm{mL}$ pressure tube equipped with a stirring bar was charged with 2-fluoro-4'-methoxy-[1,1'-biphenyl]-3-carbonitrile (0.5 mmol, $113.6 \mathrm{mg}), 2$-aminophenol (0.75 mmol, $81.8 \mathrm{mg}), \mathrm{K}_{3} \mathrm{PO}_{4}$ (2 equiv., $1 \mathrm{mmol}, 212.3 \mathrm{mg}$ ) and DMF (1 mL). The reaction was kept at $100{ }^{\circ} \mathrm{C}$ (oil bath) for $6 \mathrm{~h}$, cooled to room temperature, quenched with distilled water and extracted with ethyl acetate $(3 \times 10 \mathrm{~mL})$. The combined organic phases were dried with $\mathrm{MgSO}_{4}$, and the product was purified by column chromatography (ethyl acetate/hexanes $=7: 3$ ) as a darkred oil $(84 \%, 134 \mathrm{mg})$.

${ }^{1}$ H-NMR (400 MHz, DMSO-d $): \delta[\mathbf{p p m}]=7.67(\mathrm{dd}, J=7.8,1.7 \mathrm{~Hz}, 1 \mathrm{H}), 7.61(\mathrm{dd}, J=7.8$, $1.7 \mathrm{~Hz}, 1 \mathrm{H}), 7.44-7.40(\mathrm{~m}, 2 \mathrm{H}), 7.35(\mathrm{t}, J=7.8 \mathrm{~Hz}, 1 \mathrm{H}), 6.89-6.85(\mathrm{~m}, 2 \mathrm{H}), 6.85-6.79(\mathrm{~m}$, 2H), 6.56 (ddd, $J=8.8,6.9,2.0 \mathrm{~Hz}, 1 \mathrm{H}), 6.27$ (dd, $J=8.2,1.0 \mathrm{~Hz}, 1 \mathrm{H}), 4.34(\mathrm{bs}, 2 \mathrm{H}), 3.76$ (s, $3 \mathrm{H})$.

20 Feng, J.-B.; Wu, X.-F. Base-Promoted Synthesis of Dibenzoxazepinamines and Quinazolinimines under Metal-Free Conditions. Green Chem. 2015, 17 (9), 4522-4526. 
${ }^{13} \mathbf{C}\left\{{ }^{1} \mathbf{H}\right\}$ NMR (101 MHz, DMSO-d6): $\boldsymbol{\delta}[\mathrm{ppm}]=159.6,154.2,146.3,136.9,135.8,132.7$,

$130.0(2 \mathrm{C}), 127.9,125.8,123.5,119.6,117.4,115.7,114.2,114.1(2 \mathrm{C}), 108.6,55.4$.

HRMS (ESI-TOF) m/z: [M+H] $]^{+}$Calcd for $\mathrm{C}_{20} \mathrm{H}_{17} \mathrm{~N}_{2} \mathrm{O}_{2}$ 317.1285; Found 317.1268.

\section{COMPUTATIONAL STUDY: $\mathrm{pK}$ a CALCULATIONS}

The $\mathrm{p} K_{\mathrm{a}}$ calculations were performed using hypothetical reactions which combine computational and experimental values, as suggested in some studies. ${ }^{21}$ The deprotonated and neutral compounds had their geometries optimized in B3LYP/6-31+G(d,p) level in Gaussian 03 software. ${ }^{22}$ The vibrational frequencies were calculated with the same model $^{23}$ where all values were positive, which suggest the minimum in the potential energy surface. The solvent system (THF) was employed using the PCM model ${ }^{24}$ at B3LYP/6-311++G(d,p) and the pyridine was the reference compound to the $\mathrm{p} K_{\mathrm{a}}$ value (40.2 in THF). The $\mathrm{p} K_{\mathrm{a}}$ values are in agreement with the experimental observations (Tables S2 and S3 - section VI).

Table S2 - Solvation Gibbs energies $\left(G_{s o l v}\right)$, in Hartree, obtained by PCM/B3LYP/6$311++\mathrm{G}(\mathrm{d}, \mathrm{p}) / / \mathrm{B} 3 \mathrm{LYP} / 6-31+\mathrm{G}(\mathrm{d}, \mathrm{p})$ and $\mathrm{p} K_{\mathrm{a}}$ values.

\begin{tabular}{|c|c|c|}
\hline Compound & \multicolumn{1}{|c|}{$\boldsymbol{G}_{\text {solv }}$} & pKa \\
\hline & Neutral (-248.355300) & $\mathrm{H} 4=40.2$ \\
\cline { 2 - 2 } & Deprotonated $\underline{\mathbf{4}}(-247.795888)$ & \\
\hline & Neutral (-324.582271) & $\mathrm{H} 2=37.4$ \\
\hline
\end{tabular}

${ }^{21}$ Casasnovas, R.; Ortega-Castro, J.; Frau, J.; Donoso, J.; Muñoz, F. Theoretical p $K_{\text {a }}$ Calculations with Continuum Model Solvents, Alternative Protocols to Thermodynamic Cycles. Int. J. Quantum Chem. 2014, 114 (20), 1350-1363.

${ }^{22}$ Frisch, M. J.; Trucks, G. W.; Schlegel, H. B.; Scuseria, G. E.; Robb, M. A.; Cheeseman, J. R.; Montgomery, Jr., J. A.; Vreven, T.; Kudin, K. N.; Burant, J. C.; Millam, J. M.; Iyengar, S. S.; Tomasi, J.; Barone, V.; Mennucci, B.; Cossi, M.; Scalmani, G.; Rega, N.; Petersson, G. A.; Nakatsuji, H.; Hada, M.; Ehara, M.; Toyota, K.; Fukuda, R.; Hasegawa, J.; Ishida, M.; Nakajima, T.; Honda, Y.; Kitao, O.; Nakai, H.; Klene, M.; Li, X.; Knox, J. E.; Hratchian, H. P.; Cross, J. B.; Bakken, V.; Adamo, C.; Jaramillo, J.; Gomperts, R.; Stratmann, R. E.; Yazyev, O.; Austin, A. J.; Cammi, R.; Pomelli, C.; Ochterski, J. W.; Ayala, P. Y.; Morokuma, K.; Voth, G. A.; Salvador, P.; Dannenberg, J. J.; Zakrzewski, V. G.; Dapprich, S.; Daniels, A. D.; Strain, M. C.; Farkas, O.; Malick, D. K.; Rabuck, A. D.; Raghavachari, K.; Foresman, J. B.; Ortiz, J. V.; Cui, Q.; Baboul, A. G.; Clifford, S.; Cioslowski, J.; Stefanov, B. B.; Liu, G.; Liashenko, A.; Piskorz, P.; Komaromi, I.; Martin, R. L.; Fox, D. J.; Keith, T.; Al-Laham, M. A.; Peng, C. Y.; Nanayakkara, A.; Challacombe, M.; Gill, P. M. W.; Johnson, B.; Chen, W.; Wong, M. W.; Gonzalez, C.; and Pople, J. A.; Gaussian 03, Gaussian, Inc., Wallingford CT, 2004.

${ }^{23}$ Becke, A. D. Density-Functional Exchange-Energy Approximation with Correct Asymptotic Behavior. Phys. Rev. A 1988, 38 (6), 3098-3100.

${ }^{24}$ Barone, V. Vibrational Zero-Point Energies and Thermodynamic Functions beyond the Harmonic Approximation. J. Chem. Phys. 2004, 120 (7), 3059-3065. 


\begin{tabular}{|c|c|c|}
\hline \multirow{3}{*}{$\underbrace{C N}_{4}$} & \multirow{2}{*}{\begin{tabular}{|l} 
Deprotonated $\underline{\mathbf{2}}(-324.028840)$ \\
Deprotonated $\underline{\mathbf{3}}(-324.023759)$
\end{tabular}} & \multirow{3}{*}{$\begin{array}{l}\mathrm{H} 3=39.8 \\
\mathrm{H} 4=39.9\end{array}$} \\
\hline & & \\
\hline & Deprotonated $\underline{4}(-324.023544)$ & \\
\hline & Neutral $(-423.846520)$ & $\mathrm{H} 3=31.2$ \\
\hline & Deprotonated $\underline{\mathbf{3}}(-423.306607)$ & $\mathrm{H} 4=36.7$ \\
\hline & Deprotonated $\underline{4}(-423.294619)$ & $\mathrm{H} 5=37.9$ \\
\hline & Deprotonated $\underline{\mathbf{5}}(-423.292130)$ & $\mathrm{H} 6=34.3$ \\
\hline & Deprotonated $\underline{\mathbf{6}}(-423.299914)$ & \\
\hline & Neutral (-423.848337) & $\mathrm{H} 2=28.9$ \\
\hline & Deprotonated $\underline{\mathbf{2}}(-423.313463)$ & $\mathrm{H} 4=31.3$ \\
\hline & Deprotonated $\underline{4}(-423.308176)$ & $\mathrm{H} 5=36.3$ \\
\hline & Deprotonated $\underline{\mathbf{5}}(-423.296727)$ & $\mathrm{H} 6=35.4$ \\
\hline & Deprotonated $\underline{\mathbf{6}}(-423.299267)$ & \\
\hline & Neutral (-423.849942) & $\mathrm{H} 2=34.2$ \\
\hline & Deprotonated $\underline{\mathbf{2}}(-423.303580)$ & $\mathrm{H} 3=31.1$ \\
\hline & Deprotonated $\underline{\mathbf{3}}(-423.310234)$ & \\
\hline & Neutral (-439.893292) & $\mathrm{H} 4=28.9$ \\
\hline & Deprotonated $\underline{\mathbf{4}}(-439.358408)$ & $\mathrm{H} 5=32.8$ \\
\hline 1 & Deprotonated $\underline{\mathbf{5}}(-439.349881)$ & $\mathrm{H} 6=35.1$ \\
\hline & Deprotonated $\underline{\mathbf{6}}(-439.344861)$ & \\
\hline & Neutral (-439.893001) & $\mathrm{H} 3=25.7$ \\
\hline & Deprotonated $\underline{\mathbf{3}}(-439.365130)$ & $\mathrm{H} 5=30.6$ \\
\hline 1 & Deprotonated $\underline{\mathbf{5}}(-439.354546)$ & $\mathrm{H} 6=35.3$ \\
\hline & Deprotonated $\underline{\mathbf{6}}(-439.344165)$ & \\
\hline & Neutral (-439.884033) & $\mathrm{H} 3=29.4$ \\
\hline & Deprotonated $\underline{\mathbf{3}}(-439.348086)$ & $\mathrm{H} 4=25.9$ \\
\hline 1 & Deprotonated $\underline{4}(-439.355624)$ & $\mathrm{H} 6=31.4$ \\
\hline & Deprotonated $\underline{\mathbf{6}}(-439.343673)$ & \\
\hline & Neutral $(-523.108392)$ & $\mathrm{H} 2=25.4$ \\
\hline & Deprotonated $\underline{\mathbf{2}}(-522.581180)$ & $\mathrm{H} 5=27.7$ \\
\hline
\end{tabular}




\begin{tabular}{|c|c|c|}
\hline \multirow{2}{*}{$\mathrm{CCN}_{1}^{\mathrm{N}}$} & Deprotonated $\underline{\mathbf{5}}(-522.576255)$ & \multirow[t]{2}{*}{$\mathrm{H} 6=32.5$} \\
\hline & Deprotonated $\underline{6}(-522.565647)$ & \\
\hline \multirow{3}{*}{$\underbrace{C N}_{4}$} & Neutral $(-523.112559)$ & \multirow{3}{*}{$\begin{array}{l}\mathrm{H} 2=27.0 \\
\mathrm{H} 4=22.8\end{array}$} \\
\hline & Deprotonated $\underline{\mathbf{2}}(-522.581814)$ & \\
\hline & Deprotonated $\underline{4}(-522.590933)$ & \\
\hline \multirow{4}{*}{$\mathrm{F}$} & Neutral $(-523.112488)$ & \multirow{4}{*}{$\begin{array}{l}\mathrm{H} 3=22.6 \\
\mathrm{H} 5=29.4 \\
\mathrm{H} 6=31.1\end{array}$} \\
\hline & Deprotonated $\underline{\mathbf{3}}(-522.591403)$ & \\
\hline & Deprotonated $\underline{\mathbf{5}}(-522.576637)$ & \\
\hline & Deprotonated $\underline{6}(-522.572945)$ & \\
\hline
\end{tabular}

Equilibrium geometries (in xyz), charge and multiplicity for compounds obtained in B3LYP/6-31+G(d,p) model.

Only positive vibrational frequencies were observed for all species.

01
C
C
C
C
C
C
C
$\mathrm{N}$
$\mathrm{H}$
$\mathrm{H}$
$\mathrm{H}$
$\mathrm{H}$
$\mathrm{H}$<smiles>N#Cc1ccccc1</smiles>

$\begin{array}{llll}\text { C } & 0.09107600 & -1.21861100 & -0.00001400\end{array}$

C $\quad 1.48530200-1.21197600-0.00005400$

$\begin{array}{llll}\text { C } & 2.18284000 & -0.00000100 & 0.00005800\end{array}$

$\begin{array}{llll}\mathrm{C} & 1.48530200 & 1.21197500 & 0.00002000\end{array}$

$\begin{array}{llll}\mathrm{C} & 0.09107800 & 1.21861300 & -0.00012300\end{array}$

C $\quad-0.61061300 \quad 0.00000000-0.00003400$

$\begin{array}{llll}\mathrm{C} & -2.04675800 & 0.00000100 & -0.00001300\end{array}$

$\begin{array}{llll}\mathrm{N} & -3.21080500 & 0.00000000 & 0.00010900\end{array}$

$\mathrm{H} \quad-0.45820500 \quad-2.15402200 \quad 0.00005800$

$\mathrm{H} \quad 2.02702800-2.15260200 \quad-0.00023600$

$\begin{array}{llll}\mathrm{H} & 3.26861600 & -0.00000100 & 0.00044400\end{array}$

$\mathrm{H} \quad 2.02703100 \quad 2.15260000 \quad 0.00033400$

$\mathrm{H} \quad-0.45820200 \quad 2.15402400 \quad-0.00039500$<smiles>N#Cc1ccccc1</smiles>

$-11$
C
$0.07095000-1.36340200$
$-0.00004200$
C
$1.48573600-1.21318700$
0.00002000
C
$2.16561300 \quad 0.01161700$
0.00009500
C
$1.44686000 \quad 1.21846800$
0.00008700 


$\begin{array}{lrrc}\mathrm{C} & 0.05550000 & 1.16396000 & 0.00001600 \\ \mathrm{C} & -0.58866100 & -0.09854800 & -0.00003300 \\ \mathrm{C} & -2.03395200 & -0.07104700 & -0.00007100 \\ \mathrm{~N} & -3.20136400 & -0.01013900 & -0.00010400 \\ \mathrm{H} & 2.10651700 & -2.11569700 & 0.00000800 \\ \mathrm{H} & 3.25815400 & 0.03850300 & 0.00016000 \\ \mathrm{H} & 1.96243800 & 2.17696900 & 0.00013200 \\ \mathrm{H} & -0.52983300 & 2.08403000 & -0.00000600\end{array}$

$-11$

$\begin{array}{lrrr}\mathrm{C} & 0.12069500 & -1.24433800 & 0.00003800 \\ \mathrm{C} & 1.53100100 & -1.37321900 & 0.00011700 \\ \mathrm{C} & 2.18697800 & -0.11341000 & 0.00012100 \\ \mathrm{C} & 1.54073800 & 1.13722400 & 0.00005000 \\ \mathrm{C} & 0.14844100 & 1.20663600 & -0.00002500 \\ \mathrm{C} & -0.57237300 & -0.00501100 & -0.00003000 \\ \mathrm{C} & -2.00523500 & 0.02686100 & -0.00010500 \\ \mathrm{~N} & -3.17479700 & 0.02846800 & -0.00016800 \\ \mathrm{H} & 3.28263500 & -0.08865500 & 0.00017300 \\ \mathrm{H} & 2.12084900 & 2.06255700 & 0.00005400 \\ \mathrm{H} & -0.37570700 & 2.15886000 & -0.00008000 \\ \mathrm{H} & -0.50566800 & -2.14048900 & 0.00003100\end{array}$

$\begin{array}{lrrr}-11 & & & \\ \mathrm{C} & -0.14844300 & 1.21264700 & -0.00000100 \\ \mathrm{C} & -1.54582600 & 1.18236400 & 0.00000000 \\ \mathrm{C} & -2.34143400 & 0.00001700 & 0.00002300 \\ \mathrm{C} & -1.54587100 & -1.18234500 & 0.00003000 \\ \mathrm{C} & -0.14846100 & -1.21268100 & 0.00000500 \\ \mathrm{C} & 0.57490900 & -0.00001400 & -0.00000400 \\ \mathrm{C} & 2.00265800 & -0.00005300 & -0.00001800 \\ \mathrm{~N} & 3.17258800 & 0.00002700 & -0.00003400 \\ \mathrm{H} & -2.04631600 & -2.15664300 & 0.00004300 \\ \mathrm{H} & 0.39960100 & -2.15571600 & -0.00001000 \\ \mathrm{H} & 0.39968900 & 2.15578500 & 0.00000300 \\ \mathrm{H} & -2.04628300 & 2.15677100 & -0.00000700\end{array}$




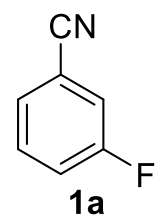

01

$\begin{array}{lrrr}\mathrm{C} & 0.60326200 & 1.41262800 & -0.00003200 \\ \mathrm{C} & -0.73904500 & 1.79152000 & 0.00002400 \\ \mathrm{C} & -1.75161200 & 0.82843400 & 0.00007100 \\ \mathrm{C} & -1.38874600 & -0.51420600 & 0.00006400 \\ \mathrm{C} & -0.06704200 & -0.93505400 & 0.00001000 \\ \mathrm{C} & 0.93825000 & 0.04728000 & -0.00003900 \\ \mathrm{C} & 2.31765900 & -0.35216700 & -0.00009800 \\ \mathrm{~N} & 3.43644200 & -0.67176100 & -0.00014800 \\ \mathrm{H} & 1.38998900 & 2.15856200 & -0.00007200 \\ \mathrm{H} & -1.00172100 & 2.84444200 & 0.00003100 \\ \mathrm{H} & -2.80115500 & 1.10143800 & 0.00011200 \\ \mathrm{~F} & -2.36569900 & -1.45343100 & 0.00010700 \\ \mathrm{H} & 0.17272600 & -1.99184200 & 0.00000700\end{array}$<smiles>N#Cc1cccc(F)c1</smiles>

$-11$

$\begin{array}{lrrr}\text { C } & 0.63622100 & 1.35190600 & -0.00003300 \\ \mathrm{C} & -0.68832100 & 1.77908800 & 0.00002200 \\ \mathrm{C} & -1.71369800 & 0.81851500 & 0.00007000 \\ \mathrm{C} & -1.34972000 & -0.52497900 & 0.00006100 \\ \mathrm{C} & -0.07325100 & -1.06680400 & 0.00000800 \\ \mathrm{C} & 0.90739800 & -0.04119400 & -0.00003900 \\ \mathrm{C} & 2.30510000 & -0.41550500 & -0.00009800 \\ \mathrm{~N} & 3.44276700 & -0.68083600 & -0.00014700 \\ \mathrm{H} & 1.45061800 & 2.07459600 & -0.00007000 \\ \mathrm{H} & -0.92986300 & 2.83946700 & 0.00002900 \\ \mathrm{H} & -2.76126100 & 1.11604200 & 0.00011400 \\ \mathrm{~F} & -2.44458300 & -1.40782400 & 0.00011100\end{array}$

$-11$
$\mathrm{C}$
$-0.58471200$
$1.41955500 \quad 0.00003100$
C
0.76298900
$1.80210000-0.00002500$
C
1.86214400
$0.90518400-0.00007700$
C
$\begin{array}{lll}1.43699900 & -0.41619500 & -0.00006400\end{array}$
$\begin{array}{llll}\mathrm{C} & 0.13497700 & -0.90078400 & -0.00001000\end{array}$
$\mathrm{C}$
$-0.91128000$
0.04833400
0.00003900 


$\begin{array}{lccc}\mathrm{C} & -2.27483000 & -0.37794600 & 0.00009700 \\ \mathrm{~N} & -3.39161500 & -0.72342500 & 0.00014500 \\ \mathrm{H} & -1.38487800 & 2.15841400 & 0.00006900 \\ \mathrm{H} & 0.95816300 & 2.87911200 & -0.00002900 \\ \mathrm{~F} & 2.41029900 & -1.43210200 & -0.00011100 \\ \mathrm{H} & -0.08239300 & -1.96612000 & -0.00000500\end{array}$

$-11$

$\begin{array}{lrrr}\mathrm{C} & 0.59030600 & 1.43729100 & -0.00003200 \\ \mathrm{C} & -0.73439300 & 1.94361700 & 0.00002300 \\ \mathrm{C} & -1.71815400 & 0.91941000 & 0.00007000 \\ \mathrm{C} & -1.39913100 & -0.43690100 & 0.00006200 \\ \mathrm{C} & -0.10012400 & -0.91501500 & 0.00000900 \\ \mathrm{C} & 0.92022800 & 0.05970400 & -0.00004000 \\ \mathrm{C} & 2.29104800 & -0.36303100 & -0.00009700 \\ \mathrm{~N} & 3.41596900 & -0.68006500 & -0.00014500 \\ \mathrm{H} & 1.43415100 & 2.13084600 & -0.00007100 \\ \mathrm{~F} & -2.41926600 & -1.38118200 & 0.00011100 \\ \mathrm{H} & 0.11169100 & -1.97854000 & 0.00000400 \\ \mathrm{H} & -2.78291200 & 1.16832900 & 0.00011500\end{array}$

$\begin{array}{lrrr}-11 & & & \\ \mathrm{C} & 0.66649200 & 1.55377400 & -0.00003700 \\ \mathrm{C} & -0.73249300 & 1.80642400 & 0.00002100 \\ \mathrm{C} & -1.73764000 & 0.82543700 & 0.00007000 \\ \mathrm{C} & -1.35088800 & -0.51125100 & 0.00006100 \\ \mathrm{C} & -0.02229500 & -0.88357800 & 0.00000600 \\ \mathrm{C} & 0.94575200 & 0.15810400 & -0.00004100 \\ \mathrm{C} & 2.32498300 & -0.27332500 & -0.00009800 \\ \mathrm{~N} & 3.42912300 & -0.65565800 & -0.00014400 \\ \mathrm{~F} & -2.32696200 & -1.49427300 & 0.00010800 \\ \mathrm{H} & 0.25275500 & -1.93588200 & 0.00000100 \\ \mathrm{H} & -2.79802100 & 1.07613000 & 0.00011300 \\ \mathrm{H} & -1.07940800 & 2.84430500 & 0.00003000\end{array}$


<smiles>N#Cc1ccc(F)cc1</smiles>

$\begin{array}{lrrr}01 & & & \\ \mathrm{C} & 0.36184800 & 1.21795800 & -0.00002900 \\ \mathrm{C} & -1.03094600 & 1.22130200 & 0.00004500 \\ \mathrm{C} & -1.69713000 & -0.00002500 & 0.00010400 \\ \mathrm{C} & -1.03089300 & -1.22130000 & 0.00006200 \\ \mathrm{C} & 0.36188300 & -1.21790200 & -0.00000300 \\ \mathrm{C} & 1.06580400 & 0.00003300 & -0.00003600 \\ \mathrm{C} & 2.50023800 & 0.00005200 & -0.00010700 \\ \mathrm{~N} & 3.66415100 & -0.00005300 & -0.00017400 \\ \mathrm{H} & 0.90814800 & 2.15497400 & -0.00007000 \\ \mathrm{H} & -1.59719800 & 2.14585700 & 0.00005800 \\ \mathrm{H} & 0.90824900 & -2.15495800 & -0.00002200 \\ \mathrm{H} & -1.59714300 & -2.14593400 & 0.00007900 \\ \mathrm{~F} & -3.05066000 & -0.00003000 & 0.00010500\end{array}$<smiles>N#Cc1ccc(F)cc1</smiles>

$-11$

$\begin{array}{lrrr}\mathrm{C} & -0.39847100 & -1.36494700 & -0.00002700 \\ \mathrm{C} & 1.01793600 & -1.24143500 & 0.00003600 \\ \mathrm{C} & 1.66258200 & -0.01336100 & 0.00007300 \\ \mathrm{C} & 0.99245100 & 1.20899900 & 0.00005300 \\ \mathrm{C} & -0.40000400 & 1.16007300 & -0.00000900 \\ \mathrm{C} & -1.05247000 & -0.09596500 & -0.00004600 \\ \mathrm{C} & -2.49616500 & -0.06030700 & -0.00010900 \\ \mathrm{~N} & -3.66303700 & 0.00588100 & -0.00016100 \\ \mathrm{H} & 1.65085100 & -2.13196400 & 0.00005700 \\ \mathrm{H} & -0.97404400 & 2.08686600 & -0.00002700 \\ \mathrm{H} & 1.54400100 & 2.14384500 & 0.00008300 \\ \mathrm{~F} & 3.05170000 & 0.03352700 & 0.00013200\end{array}$

$-11$<smiles>N#Cc1ccc(F)cc1</smiles>

C $\begin{array}{lll}0.33143300 & -1.23832300 & 0.00002300\end{array}$

$\begin{array}{llll}\text { C } & -1.07372100 & -1.34665000 & -0.00003300\end{array}$

$\begin{array}{llll}\text { C } & -1.67667600 & -0.10055100 & -0.00006800\end{array}$

$\begin{array}{llll}\mathrm{C} & -1.06744000 & 1.15868000 & -0.00005700\end{array}$

$\begin{array}{llll}\mathrm{C} & 0.32559100 & 1.20942100 & 0.00000300\end{array}$

C $\quad 1.04020700 \quad-0.00480900 \quad 0.00004600$ 


$\begin{array}{lrrr}\mathrm{C} & 2.47096700 & 0.01481300 & 0.00011000 \\ \mathrm{~N} & 3.64039300 & 0.01100900 & 0.00016100 \\ \mathrm{H} & 0.85155400 & 2.15985200 & 0.00001600 \\ \mathrm{H} & -1.66056400 & 2.07124500 & -0.00009400 \\ \mathrm{~F} & -3.08015600 & -0.03569100 & -0.00013900 \\ \mathrm{H} & 0.94549600 & -2.14242500 & 0.00006000\end{array}$

01<smiles></smiles>

$\mathrm{C}$

$0.33396000-1.49529000$

0.00000700

C

$1.71761300-1.33877700$

0.00006100

C

$2.27778000-0.05633900$

0.00008700

C

$1.45975200 \quad 1.07557500$

0.00006000

C

$0.08283700 \quad 0.90279800$

0.00000800

C

$\begin{array}{lll}-0.50960300 & -0.36793700 & -0.00002100\end{array}$

C

$\begin{array}{llll}-1.93382000 & -0.51356200 & -0.00007800\end{array}$

$\mathrm{N}$

$\begin{array}{lll}-3.08811000 & -0.65838200 & -0.00012600\end{array}$

$\mathrm{H}$

$\begin{array}{lll}-0.11371200 & -2.48326400 & -0.00001600\end{array}$

$\mathrm{H}$

$\begin{array}{lll}2.35836600 & -2.21418000 & 0.00008500\end{array}$

$\mathrm{H}$

$3.35636200 \quad 0.06628500$

0.00012700

$\mathrm{H}$

$\begin{array}{lll}1.86825500 & 2.07999400 & 0.00007800\end{array}$

F

$-0.71373500$

1.99122600

$-0.00001600$

$-11$

$\begin{array}{lrrl}\mathrm{C} & 0.40994800 & -1.46794300 & 0.00000700 \\ \mathrm{C} & 1.78252800 & -1.24569400 & 0.00006200 \\ \mathrm{C} & 2.27928200 & 0.07575300 & 0.00008900 \\ \mathrm{C} & 1.47870600 & 1.23929700 & 0.00006400 \\ \mathrm{C} & 0.13130900 & 0.93342800 & 0.00001100 \\ \mathrm{C} & -0.45597300 & -0.34976100 & -0.00002000 \\ \mathrm{C} & -1.87217000 & -0.55053700 & -0.00007600 \\ \mathrm{~N} & -3.02456400 & -0.74297400 & -0.00012100 \\ \mathrm{H} & -0.00867800 & -2.47031000 & -0.00001400 \\ \mathrm{H} & 2.46518300 & -2.09619400 & 0.00008300 \\ \mathrm{H} & 3.36944700 & 0.18272800 & 0.00013100 \\ \mathrm{~F} & -0.79731000 & 1.97526000 & -0.00001900\end{array}$


<smiles>N#Cc1cc[c-]cc1F</smiles>

$-11$

$\begin{array}{lrrr}\mathrm{C} & 0.38179900 & -1.49220700 & 0.00000900 \\ \mathrm{C} & 1.76367900 & -1.31422600 & 0.00006300 \\ \mathrm{C} & 2.42832200 & -0.05080200 & 0.00009500 \\ \mathrm{C} & 1.52271800 & 1.04766200 & 0.00006400 \\ \mathrm{C} & 0.14634600 & 0.89273700 & 0.00000900 \\ \mathrm{C} & -0.47713400 & -0.36574200 & -0.00002000 \\ \mathrm{C} & -1.89371900 & -0.51012600 & -0.00007600 \\ \mathrm{~N} & -3.05237100 & -0.66379100 & -0.00012200 \\ \mathrm{H} & -0.06746300 & -2.48581200 & -0.00001300 \\ \mathrm{H} & 2.36704100 & -2.22683700 & 0.00008300 \\ \mathrm{~F} & -0.67304000 & 2.00437900 & -0.00001800 \\ \mathrm{H} & 1.89230600 & 2.07600700 & 0.00008200\end{array}$<smiles>N#Cc1c[c-]ccc1F</smiles>

$-11$

$\begin{array}{lccc}\mathrm{C} & 0.33533500 & -1.53182500 & 0.00000900 \\ \mathrm{C} & 1.74681900 & -1.52887000 & 0.00006300 \\ \mathrm{C} & 2.28269600 & -0.21458700 & 0.00008800 \\ \mathrm{C} & 1.52853300 & 0.97755600 & 0.00006200 \\ \mathrm{C} & 0.14943200 & 0.88186800 & 0.00000800 \\ \mathrm{C} & -0.48715200 & -0.36445400 & -0.00002000 \\ \mathrm{C} & -1.91243600 & -0.46132200 & -0.00007600 \\ \mathrm{~N} & -3.07257700 & -0.60185000 & -0.00012100 \\ \mathrm{H} & -0.21019100 & -2.47841000 & -0.00001500 \\ \mathrm{~F} & -0.61145500 & 2.02903600 & -0.00001800 \\ \mathrm{H} & 1.99224200 & 1.96291300 & 0.00008300 \\ \mathrm{H} & 3.36972400 & -0.08307400 & 0.00013000\end{array}$<smiles>N#Cc1[c-]cccc1F</smiles>

$-11$

$\begin{array}{llll}\mathrm{C} & 0.33859200 & -1.62896100 & 0.00000500 \\ \mathrm{C} & 1.72895000 & -1.33468500 & 0.00006000 \\ \mathrm{C} & 2.27428500 & -0.04592800 & 0.00008800 \\ \mathrm{C} & 1.43313700 & 1.07929400 & 0.00006100 \\ \mathrm{C} & 0.06989200 & 0.84447600 & 0.00000700 \\ \mathrm{C} & -0.47203000 & -0.45347100 & -0.00002100\end{array}$




$\begin{array}{lccc}\mathrm{C} & -1.90779000 & -0.58252900 & -0.00007700 \\ \mathrm{~N} & -3.07079400 & -0.68647200 & -0.00012200 \\ \mathrm{~F} & -0.76676000 & 1.94482400 & -0.00002000 \\ \mathrm{H} & 1.81112400 & 2.09690400 & 0.00008100 \\ \mathrm{H} & 3.35667400 & 0.10333500 & 0.00013000 \\ \mathrm{H} & 2.43839800 & -2.16753100 & 0.00008300\end{array}$

$\begin{array}{lccc}\mathrm{C} & 1.67249600 & -0.00352300 & 0.00000000 \\ \mathrm{C} & 0.94425400 & -1.19402400 & 0.00000000 \\ \mathrm{~N} & -0.38820200 & -1.20213900 & 0.00000000 \\ \mathrm{C} & -1.02356800 & -0.01690700 & 0.00000000 \\ \mathrm{C} & -0.36027000 & 1.21966100 & 0.00000000 \\ \mathrm{C} & 1.03318200 & 1.22740000 & 0.00000000 \\ \mathrm{~F} & 3.02071700 & -0.05994800 & 0.00000000 \\ \mathrm{C} & -2.46664300 & -0.05093400 & 0.00000000 \\ \mathrm{~N} & -3.62905600 & -0.03957500 & 0.00000000 \\ \mathrm{H} & 1.45757600 & -2.15171900 & 0.00000000 \\ \mathrm{H} & -0.92588600 & 2.14490100 & 0.00000100 \\ \mathrm{H} & 1.60596200 & 2.14831100 & 0.00000000 \\ & & & \\ & & & \end{array}$

$-11$

$\begin{array}{lrrr}\mathrm{C} & -0.13533900 & -1.64838600 & 0.00000000 \\ \mathrm{C} & -1.35986000 & -0.95602000 & 0.00000000 \\ \mathrm{~N} & -1.22945800 & 0.41535600 & 0.00000000 \\ \mathrm{C} & 0.00000000 & 0.99277600 & 0.00000000 \\ \mathrm{C} & 1.21328800 & 0.29444500 & 0.00000000 \\ \mathrm{C} & 1.13771800 & -1.10240500 & 0.00000000 \\ \mathrm{~F} & -0.18430200 & -3.03660500 & 0.00000000 \\ \mathrm{C} & 0.04017300 & 2.43746500 & 0.00000000 \\ \mathrm{~N} & 0.09874100 & 3.60280000 & 0.00000000 \\ \mathrm{H} & 2.16503800 & 0.81778700 & 0.00000000 \\ \mathrm{H} & 2.03281900 & -1.72268300 & 0.00000000\end{array}$

$-11$<smiles>N#Cc1c[c-]c(F)cn1</smiles>

$\begin{array}{lrrr}\mathrm{C} & 1.65108100 & 0.09719700 & 0.00000000 \\ \mathrm{C} & 0.98263100 & -1.12929900 & 0.00000000 \\ \mathrm{~N} & -0.35384800 & -1.20246700 & 0.00000000 \\ \mathrm{C} & -0.99226200 & -0.01214000 & -0.00000100 \\ \mathrm{C} & -0.33126700 & 1.23415000 & 0.00000000 \\ \mathrm{C} & 1.07750200 & 1.35554900 & 0.00000000\end{array}$




$\begin{array}{lrrr}\mathrm{F} & 3.04632400 & -0.02138300 & -0.00000100 \\ \mathrm{C} & -2.43545500 & -0.06208700 & -0.00000100 \\ \mathrm{~N} & -3.60234600 & -0.04876500 & 0.00000000 \\ \mathrm{H} & 1.52748300 & -2.07382800 & 0.00000000 \\ \mathrm{H} & -0.96441500 & 2.12468300 & 0.00000000\end{array}$

$-11$

$\begin{array}{lrrr}\mathrm{C} & 1.63635500 & 0.01200400 & 0.00000000 \\ \mathrm{C} & 0.90681700 & -1.17603900 & 0.00000100 \\ \mathrm{~N} & -0.42624700 & -1.15629600 & 0.00000000 \\ \mathrm{C} & -1.00757200 & 0.07533800 & 0.00000300 \\ \mathrm{C} & -0.39962800 & 1.35675500 & 0.00000100 \\ \mathrm{C} & 1.01618300 & 1.24942800 & 0.00000000 \\ \mathrm{~F} & 3.01844800 & -0.08912600 & -0.00000200 \\ \mathrm{C} & -2.46130200 & 0.01171900 & 0.00000000 \\ \mathrm{~N} & -3.62706800 & -0.03960500 & -0.00000200 \\ \mathrm{H} & 1.41117100 & -2.14085500 & 0.00000000 \\ \mathrm{H} & 1.65088700 & 2.13906700 & 0.00000200\end{array}$

01

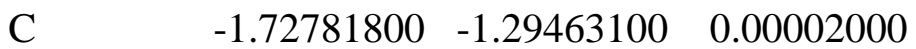

$\begin{array}{llll}\text { C } & -2.21387300 & 0.01332500 & -0.00003300\end{array}$

$\begin{array}{llll}\text { C } & -0.12345300 & 0.88882200 & -0.00003300\end{array}$

$\begin{array}{llll}\mathrm{C} & 0.50037700 & -0.37300200 & 0.00002100\end{array}$

$\begin{array}{llll}\text { C } & -0.34819600 & -1.49023800 & 0.00004800\end{array}$

$\mathrm{H} \quad-2.41165200 \quad-2.13590300 \quad 0.00004000$

$\mathrm{H} \quad-3.28200900 \quad 0.21244400 \quad-0.00005600$

$\mathrm{N} \quad \begin{array}{llll}-1.41712700 & 1.09443400 & -0.00006000\end{array}$

F $\quad \begin{array}{llll}0.65662900 & 1.97906800 & -0.00006000\end{array}$

$\begin{array}{llll}\mathrm{H} & 0.08136700 & -2.48638600 & 0.00008900\end{array}$

$\begin{array}{llll}\mathrm{C} & 1.92404700 & -0.50682300 & 0.00004600\end{array}$

$\mathrm{N} \quad 3.07943100 \quad-0.64107500 \quad 0.00006800$

$\begin{array}{lrrr}-11 & & & \\ \mathrm{C} & -1.76984600 & -1.26597100 & 0.00001900 \\ \mathrm{C} & -2.38486300 & 0.03375700 & -0.00003600 \\ \mathrm{C} & -0.18403500 & 0.87628800 & -0.00003400 \\ \mathrm{C} & 0.46876300 & -0.36954200 & 0.00002000 \\ \mathrm{C} & -0.40100900 & -1.48507400 & 0.00004700 \\ \mathrm{H} & -2.42862900 & -2.13463800 & 0.00004000 \\ \mathrm{~N} & -1.46531300 & 1.09105900 & -0.00006000\end{array}$




$\begin{array}{lllr}\mathrm{F} & 0.63045700 & 1.98767800 & -0.00006100 \\ \mathrm{H} & 0.02891400 & -2.48673200 & 0.00008800 \\ \mathrm{C} & 1.88423100 & -0.50768900 & 0.00004600 \\ \mathrm{~N} & 3.04333500 & -0.65653700 & 0.00006800\end{array}$

$\begin{array}{cccc}-11 & & & \\ \mathrm{C} & -1.76055100 & -1.47938900 & 0.00002300 \\ \mathrm{C} & -2.22492300 & -0.14588600 & -0.00003100 \\ \mathrm{C} & -0.19255900 & 0.86287200 & -0.00003300 \\ \mathrm{C} & 0.47699100 & -0.36772300 & 0.00002000 \\ \mathrm{C} & -0.35163200 & -1.52547900 & 0.00004700 \\ \mathrm{~N} & -1.48706800 & 1.00674400 & -0.00005900 \\ \mathrm{~F} & 0.55630500 & 2.01423100 & -0.00006100 \\ \mathrm{H} & 0.17444000 & -2.48289000 & 0.00008900 \\ \mathrm{C} & 1.90135800 & -0.45294400 & 0.00004600 \\ \mathrm{~N} & 3.06204000 & -0.58571300 & 0.00006900 \\ \mathrm{H} & -3.29809500 & 0.05888900 & -0.00005500\end{array}$<smiles>N#Cc1c[c-]cnc1F</smiles>

$\begin{array}{crrr}-11 & & & \\ \mathrm{C} & -1.73573500 & -1.28785800 & 0.00002200 \\ \mathrm{C} & -2.20490500 & 0.02096600 & -0.00003100 \\ \mathrm{C} & -0.11243200 & 0.82965100 & -0.00003100 \\ \mathrm{C} & 0.45787900 & -0.45763300 & 0.00002300 \\ \mathrm{C} & -0.35334300 & -1.62883000 & 0.00005300 \\ \mathrm{~N} & -1.39167300 & 1.10485700 & -0.00005900 \\ \mathrm{~F} & 0.71503300 & 1.92769500 & -0.00005800 \\ \mathrm{C} & 1.89375900 & -0.57092700 & 0.00004700 \\ \mathrm{~N} & 3.05679200 & -0.66842100 & 0.00006600 \\ \mathrm{H} & -3.27466000 & 0.24455600 & -0.00005400 \\ \mathrm{H} & -2.48780200 & -2.08107100 & 0.00003900\end{array}$<smiles>N#Cc1[c]ccnc1F</smiles>

$\begin{array}{crrr}01 & & & \\ \mathrm{C} & -0.56832500 & 1.40153500 & 0.00000600 \\ \mathrm{C} & 0.78723400 & 1.71717800 & -0.00004800 \\ \mathrm{C} & 1.38850000 & -0.46621600 & -0.00004700 \\ \mathrm{C} & 0.07499600 & -0.93330600 & 0.00000700 \\ \mathrm{C} & -0.92826900 & 0.04203600 & 0.00003400 \\ \mathrm{C} & -2.31199700 & -0.34525700 & 0.00009100 \\ \mathrm{~N} & -3.43336700 & -0.65267500 & 0.00013700 \\ \mathrm{H} & -1.32396100 & 2.17807500 & 0.00002600 \\ & & & \mathrm{~S} 52\end{array}$




$\begin{array}{lrrr}\mathrm{H} & 1.11332300 & 2.75336000 & -0.00007200 \\ \mathrm{~N} & 1.76232900 & 0.79302300 & -0.00007500 \\ \mathrm{H} & -0.14490300 & -1.99359200 & 0.00002700 \\ \mathrm{~F} & 2.37777500 & -1.37956600 & -0.00007500\end{array}$

$\begin{array}{lrrr}-11 & & & \\ \mathrm{C} & 0.59191400 & 1.34388600 & -0.00000600 \\ \mathrm{C} & -0.74612700 & 1.70014600 & 0.00004700 \\ \mathrm{C} & -1.34944700 & -0.48026400 & 0.00005000 \\ \mathrm{C} & -0.07458500 & -1.06886900 & -0.00000800 \\ \mathrm{C} & 0.89611300 & -0.04405900 & -0.00003400 \\ \mathrm{C} & 2.29945800 & -0.40112000 & -0.00009100 \\ \mathrm{~N} & 3.43986700 & -0.64943400 & -0.00013800 \\ \mathrm{H} & 1.37094700 & 2.10300300 & -0.00002700 \\ \mathrm{H} & -1.05419900 & 2.74461600 & 0.00006900 \\ \mathrm{~N} & -1.74019800 & 0.78444900 & 0.00007600 \\ \mathrm{~F} & -2.43537600 & -1.34344900 & 0.00007200\end{array}$<smiles>N#Cc1[c-]c(F)ncc1</smiles><smiles>N#Cc1c[c-]nc(F)c1</smiles>

$-11$

$\begin{array}{lrrr}\mathrm{C} & -0.56146200 & 1.41660300 & 0.00000400 \\ \mathrm{C} & 0.79023600 & 1.88758400 & -0.00005100 \\ \mathrm{C} & 1.39477100 & -0.38742100 & -0.00004800 \\ \mathrm{C} & 0.11186700 & -0.91597400 & 0.00000600 \\ \mathrm{C} & -0.90922700 & 0.05362200 & 0.00003300 \\ \mathrm{C} & -2.28577300 & -0.35749800 & 0.00009000 \\ \mathrm{~N} & -3.41228000 & -0.66382200 & 0.00013600 \\ \mathrm{H} & -1.36612800 & 2.14968700 & 0.00002600 \\ \mathrm{~N} & 1.74706000 & 0.87146400 & -0.00007500 \\ \mathrm{~F} & 2.42914000 & -1.31141400 & -0.00007600 \\ \mathrm{H} & -0.08206400 & -1.98195200 & 0.00002600\end{array}$

$-11$

$\begin{array}{lrrr}\mathrm{C} & 0.63184200 & 1.53856000 & -0.00000800 \\ \mathrm{C} & -0.77097400 & 1.73309800 & 0.00004600 \\ \mathrm{C} & -1.35125200 & -0.45763200 & 0.00004700 \\ \mathrm{C} & -0.03526600 & -0.88224400 & -0.00000700 \\ \mathrm{C} & 0.93417800 & 0.15083700 & -0.00003300 \\ \mathrm{C} & 2.31854200 & -0.27034600 & -0.00009000 \\ \mathrm{~N} & 3.42560800 & -0.63990800 & -0.00013700 \\ \mathrm{~N} & -1.75304200 & 0.79131100 & 0.00007400\end{array}$




$\begin{array}{lrrr}\mathrm{F} & -2.34619100 & -1.41599000 & 0.00007500 \\ \mathrm{H} & 0.21701900 & -1.93879000 & -0.00002700 \\ \mathrm{H} & -1.17168300 & 2.74923600 & 0.00007200\end{array}$<smiles>N#Cc1ccc(F)cc1F</smiles>

01

$\begin{array}{lrrr}\mathrm{C} & 0.31795700 & 0.89750100 & -0.00002500 \\ \mathrm{C} & -1.06013700 & 1.05831100 & 0.00003000 \\ \mathrm{C} & -1.83488700 & -0.09608500 & 0.00007700 \\ \mathrm{C} & -1.28806900 & -1.37681900 & 0.00007100 \\ \mathrm{C} & 0.09748400 & -1.50271800 & 0.00001500 \\ \mathrm{C} & 0.92847900 & -0.36588600 & -0.00003400 \\ \mathrm{C} & 2.35327800 & -0.49301400 & -0.00009100 \\ \mathrm{~N} & 3.50953500 & -0.62117800 & -0.00013700 \\ \mathrm{H} & 0.55531400 & -2.48560900 & 0.00000900 \\ \mathrm{~F} & 1.09411200 & 1.99619000 & -0.00007100 \\ \mathrm{~F} & -3.17847900 & 0.03777500 & 0.00013100 \\ \mathrm{H} & -1.50906400 & 2.04406700 & 0.00003600 \\ \mathrm{H} & -1.93831600 & -2.24362200 & 0.00010900\end{array}$

$-11$

$\begin{array}{lrrr}\mathrm{C} & 0.26250300 & 0.91931100 & -0.00002200 \\ \mathrm{C} & -1.08899300 & 1.17772400 & 0.00003100 \\ \mathrm{C} & -1.81220300 & 0.00498300 & 0.00007600 \\ \mathrm{C} & -1.32537500 & -1.31122200 & 0.00007100 \\ \mathrm{C} & 0.05398100 & -1.48638900 & 0.00001600 \\ \mathrm{C} & 0.89123400 & -0.34838700 & -0.00003300 \\ \mathrm{C} & 2.31038100 & -0.50755200 & -0.00009000 \\ \mathrm{~N} & 3.46779900 & -0.66677600 & -0.00013700 \\ \mathrm{H} & 0.49466000 & -2.47867400 & 0.00001000 \\ \mathrm{~F} & 1.15373100 & 1.98868100 & -0.00007200 \\ \mathrm{~F} & -3.21119500 & 0.07996400 & 0.00013200 \\ \mathrm{H} & -2.00124300 & -2.16251000 & 0.00011000\end{array}$<smiles>N#Cc1ccc(F)cc1F</smiles>

$-11$

$\begin{array}{lrrr}\text { C } & 0.27626900 & 0.88222300 & -0.00002300 \\ \text { C } & -1.10316000 & 0.98884600 & 0.00003200\end{array}$ 


$\begin{array}{lrrr}\mathrm{C} & -1.81945400 & -0.21609900 & 0.00007800 \\ \mathrm{C} & -1.32813800 & -1.51027600 & 0.00007500 \\ \mathrm{C} & 0.07676700 & -1.52629400 & 0.00001800 \\ \mathrm{C} & 0.91100900 & -0.36512500 & -0.00003200 \\ \mathrm{C} & 2.33340000 & -0.46966300 & -0.00008900 \\ \mathrm{~N} & 3.49379800 & -0.60674400 & -0.00013500 \\ \mathrm{H} & 0.61191300 & -2.47839200 & 0.00000900 \\ \mathrm{~F} & 1.03130300 & 2.02776800 & -0.00007000 \\ \mathrm{~F} & -3.20520100 & -0.02099000 & 0.00013200 \\ \mathrm{H} & -1.58357500 & 1.96291800 & 0.00003800\end{array}$

$-11$

$\begin{array}{lrrr}\mathrm{C} & 0.33581000 & 0.83573700 & -0.00002400 \\ \mathrm{C} & -1.03407700 & 1.04227000 & 0.00003000 \\ \mathrm{C} & -1.81454100 & -0.11429900 & 0.00007700 \\ \mathrm{C} & -1.28455600 & -1.39535700 & 0.00007100 \\ \mathrm{C} & 0.11409700 & -1.63970900 & 0.00001600 \\ \mathrm{C} & 0.90408400 & -0.44950500 & -0.00003200 \\ \mathrm{C} & 2.34045500 & -0.55059800 & -0.00009000 \\ \mathrm{~N} & 3.50527800 & -0.62948100 & -0.00013800 \\ \mathrm{~F} & 1.13918800 & 1.95604800 & -0.00007200 \\ \mathrm{~F} & -3.18892300 & 0.06912300 & 0.00013100 \\ \mathrm{H} & -1.46560400 & 2.03552800 & 0.00003600 \\ \mathrm{H} & -1.99136500 & -2.22693500 & 0.00011000\end{array}$<smiles>N#Cc1cc(F)c(F)c(F)c1</smiles>

01

$\begin{array}{lrrr}\mathrm{C} & -0.41110500 & 1.22327500 & 0.00003700 \\ \mathrm{C} & 0.97672900 & 1.18556200 & -0.00001900 \\ \mathrm{C} & 1.70410700 & 0.00017600 & -0.00006800 \\ \mathrm{C} & 0.97696200 & -1.18542400 & -0.00005900 \\ \mathrm{C} & -0.41075200 & -1.22347300 & -0.00000400 \\ \mathrm{C} & -1.10201800 & -0.00017000 & 0.00004400 \\ \mathrm{C} & -2.53805900 & -0.00028300 & 0.00010200 \\ \mathrm{~N} & -3.70124000 & -0.00017100 & 0.00014800 \\ \mathrm{H} & -0.93517700 & 2.17106600 & 0.00007400 \\ \mathrm{H} & 2.78741200 & 0.00027300 & -0.00011100 \\ \mathrm{~F} & 1.65678200 & -2.35357400 & -0.00010600 \\ \mathrm{H} & -0.93483400 & -2.17126200 & 0.00000000 \\ \mathrm{~F} & 1.65611700 & 2.35392200 & -0.00002600\end{array}$


<smiles>N#Cc1cc(F)cc(F)c1</smiles>

$\mathrm{C}$
$\mathrm{C}$
$\mathrm{C}$
$\mathrm{C}$
$\mathrm{C}$
$\mathrm{C}$
$\mathrm{C}$
$\mathrm{N}$
$\mathrm{H}$
$\mathrm{F}$
$\mathrm{H}$
$\mathrm{F}$

$-0.40389700-1.36787600$

$-0.00004100$

$0.96841800-1.17076400$

0.00001600

$\begin{array}{ll}1.66899600 & 0.03623700\end{array}$

0.00006600

$0.89639600 \quad 1.19522500$

0.00005800

$\begin{array}{lll}-0.48299300 & 1.15791500 & 0.00000300\end{array}$

$\begin{array}{lll}-1.09040500 & -0.12942000 & -0.00004500\end{array}$

$\begin{array}{llll}\text { C } & -2.53675900 & -0.13686100 & -0.00010200\end{array}$

$\begin{array}{llll}\mathrm{N} & -3.70413100 & -0.10859300 & -0.00014800\end{array}$

$\begin{array}{llll} & 2.75426200 & 0.08909300 & 0.00010900\end{array}$

$\begin{array}{llll}1.54225700 & 2.41563300 & 0.00010500\end{array}$

$\begin{array}{llll}1.06212400 & 2.07676800 & -0.00000300\end{array}$

$\begin{array}{llll}1.80421400 & -2.29479300 & 0.00002800\end{array}$<smiles>N#Cc1cc(F)c(F)c(F)c1</smiles>

$-11$

$\begin{array}{llll}\mathrm{C} & 0.36007900 & -1.21705300 & 0.00003500\end{array}$

$\begin{array}{llll}\mathrm{C} & -1.03107700 & -1.13666800 & -0.00002200\end{array}$

$\begin{array}{lllll}\text { C } & -1.81772800 & 0.00019800 & -0.00007300\end{array}$

$\begin{array}{lllll}\mathrm{C} & -1.03079200 & 1.13685800 & -0.00006100\end{array}$

$\begin{array}{lllll}\mathrm{C} & & 0.36038700 & 1.21688600 & -0.00000600\end{array}$

$\begin{array}{llll}\mathrm{C} & 1.07218200 & -0.00018400 & 0.00004300\end{array}$

$\begin{array}{llll}\mathrm{C} & 2.50209600 & -0.00040000 & 0.00010000\end{array}$

$\begin{array}{llll}\mathrm{N} & 3.67021300 & -0.00009300 & 0.00014700\end{array}$

F $\quad \begin{array}{llll}-1.66341100 & 2.38870400 & -0.00010600\end{array}$

$\begin{array}{llll}\mathrm{H} & 0.88263700 & 2.16900900 & -0.00000200\end{array}$

F $\quad-1.66403100 \quad-2.38834700 \quad-0.00002600$

$\begin{array}{llll}\mathrm{H} & 0.88197100 & -2.16940000 & 0.00007200\end{array}$<smiles>N#Cc1ccc(F)c(F)c1</smiles>

01
$\begin{array}{lll}0.77748100 & -1.38925600 & 0.00004800\end{array}$
$\begin{array}{llll}\text { C } & -0.60061600 & -1.59875500 & -0.00000800\end{array}$
$\begin{array}{llll}\mathrm{C} & -1.45616200 & -0.50396300 & -0.00005600\end{array}$
$\begin{array}{lllll}\text { C } & -0.94922500 & 0.79661600 & -0.00004800\end{array}$
$\begin{array}{llll}\mathrm{C} & 0.41732200 & 1.02326900 & 0.00000600\end{array}$
$\begin{array}{llll}\mathrm{C} & 1.28944400 & -0.08030700 & 0.00005500\end{array}$
$\begin{array}{llll}\mathrm{C} & 2.70779800 & 0.13590200 & 0.00011200\end{array}$
$\begin{array}{llll}\mathrm{N} & 3.85866700 & 0.30729500 & 0.00015900\end{array}$ 


$\begin{array}{lrrr}\mathrm{H} & 1.45710400 & -2.23370200 & 0.00008500 \\ \mathrm{~F} & -1.80631900 & 1.83450700 & -0.00009600 \\ \mathrm{H} & 0.79144600 & 2.04058800 & 0.00001100 \\ \mathrm{~F} & -2.78841100 & -0.68578100 & -0.00011000 \\ \mathrm{H} & -1.02290200 & -2.59752600 & -0.00001500\end{array}$

$\begin{array}{crrr}-11 & & & \\ \text { C } & -0.82218100 & 1.33139200 & 0.00004800 \\ \text { C } & 0.54763900 & 1.58049200 & -0.00000700 \\ \text { C } & 1.40939800 & 0.48334200 & -0.00005500 \\ \text { C } & 0.91320200 & -0.81363300 & -0.00004700 \\ \text { C } & -0.42632500 & -1.14816900 & 0.00000600 \\ \text { C } & -1.27402900 & -0.01157600 & 0.00005400 \\ \text { C } & -2.70633600 & -0.20469500 & 0.00011200 \\ \text { N } & -3.86827900 & -0.32410400 & 0.00016000 \\ \text { H } & -1.52850700 & 2.16014500 & 0.00008600 \\ \text { F } & 1.87957400 & -1.81851200 & -0.00009800 \\ \text { F } & 2.76511100 & 0.73179900 & -0.00010900 \\ \text { H } & 0.95608700 & 2.58608600 & -0.00001400\end{array}$<smiles>N#Cc1ccc(F)c(F)c1</smiles>

$\begin{array}{lrrr}-11 & & & \\ \text { C } & 0.75881200 & -1.41084900 & 0.00004600 \\ \text { C } & -0.61726500 & -1.71192500 & -0.00000800 \\ \text { C } & -1.42141800 & -0.59141900 & -0.00005400 \\ \text { C } & -0.96461200 & 0.72674500 & -0.00004800 \\ \text { C } & 0.39182600 & 1.00667300 & 0.00000600 \\ \text { C } & 1.27721800 & -0.09030400 & 0.00005500 \\ \text { C } & 2.69080300 & 0.14407500 & 0.00011200 \\ \text { N } & 3.84736200 & 0.31141300 & 0.00016000 \\ \text { H } & 1.49426000 & -2.21795800 & 0.00008600 \\ \text { F } & -1.84364700 & 1.78963000 & -0.00009700 \\ \text { F } & -2.80742500 & -0.72661700 & -0.00011000 \\ \text { H } & 0.74166900 & 2.03297400 & 0.00001000\end{array}$




$\begin{array}{lrrr}\mathrm{C} & -1.42735300 & -0.51236400 & -0.00005400 \\ \mathrm{C} & -0.90617500 & 0.78110500 & -0.00004700 \\ \mathrm{C} & 0.46233300 & 0.96591400 & 0.00000800 \\ \mathrm{C} & 1.28869900 & -0.18743500 & 0.00005600 \\ \mathrm{C} & 2.71121700 & 0.06067400 & 0.00011300 \\ \mathrm{~N} & 3.85584200 & 0.29487800 & 0.00015800 \\ \mathrm{~F} & -1.75681800 & 1.85875400 & -0.00009500 \\ \mathrm{~F} & -2.79606900 & -0.65364200 & -0.00010900 \\ \mathrm{H} & 0.86497800 & 1.97687500 & 0.00001300 \\ \mathrm{H} & -1.08636200 & -2.59406100 & -0.00001500\end{array}$




\section{CORRELATION BETWEEN THE CALCULATED $K_{a}$ VALUES AND METALATION SITES FOR THE STUDIED SUBSTRATES}

Table S3 - Metalation sites explored in this work and the ones highlighted in the literature

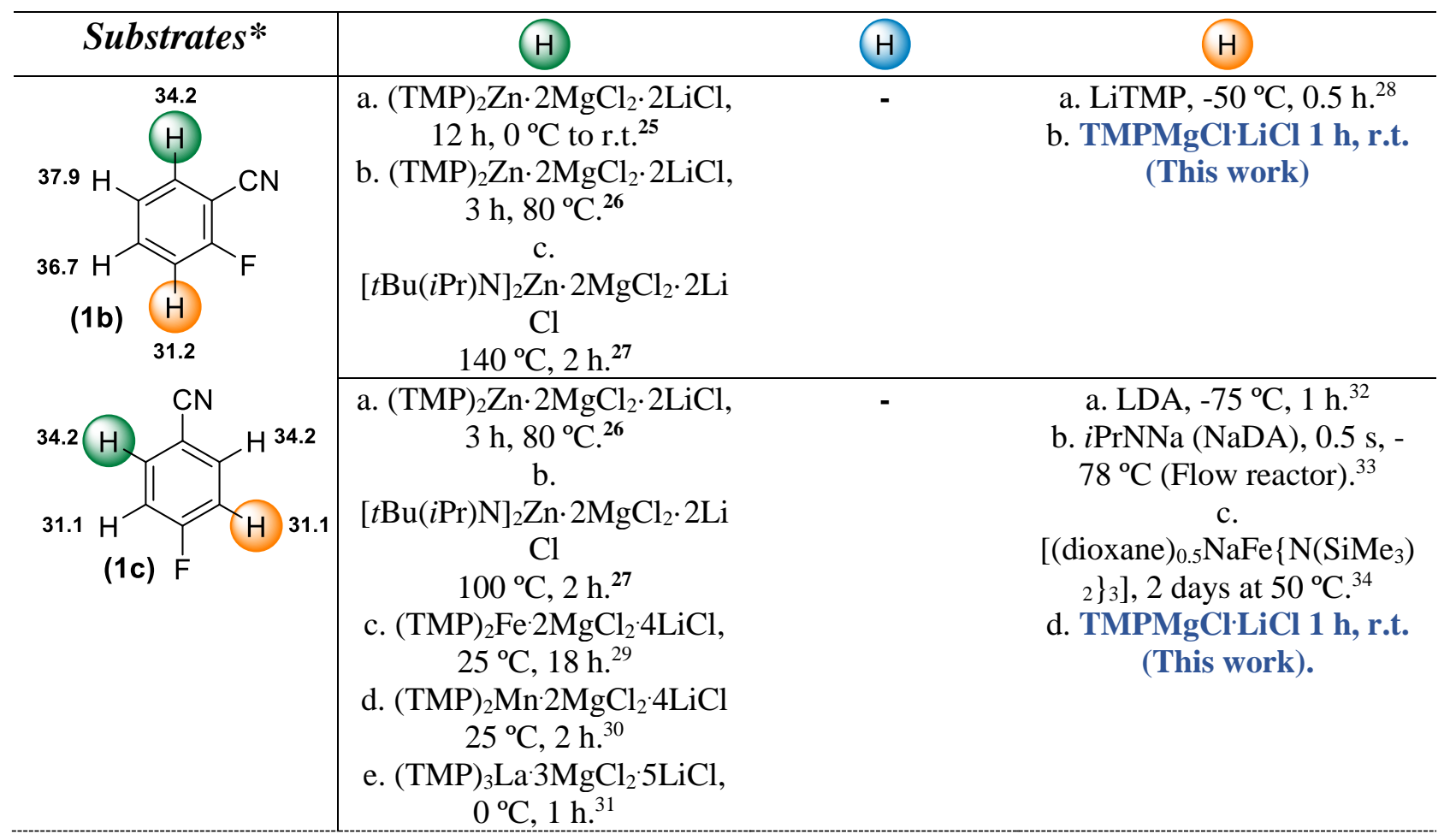

${ }^{25}$ Hammann, J. M.; Haas, D.; Knochel, P. Cobalt-Catalyzed Negishi Cross-Coupling Reactions of (Hetero)Arylzinc Reagents with Primary and Secondary Alkyl Bromides and Iodides. Angew. Chem. Int. Ed. 2015, 54 (15), 4478-4481.

26 Wunderlich, S.; Knochel, P. High Temperature Metalation of Functionalized Aromatics and Heteroaromatics Using (Tmp) ${ }_{2} \mathrm{Zn} \cdot 2 \mathrm{MgCl}_{2} \cdot 2 \mathrm{LiCl}$ and Microwave Irradiation. Org. Lett. 2008, 10 (20), 4705-4707.

${ }^{27}$ Rohbogner, C. J.; Wunderlich, S. H.; Clososki, G. C.; Knochel, P. New Mixed Li/Mg and Li/Mg/Zn Amides for the Chemoselective Metallation of Arenes and Heteroarenes. Eur. J. Org. Chem. 2009, 2009 (11), 1781-1795.

${ }^{28}$ Eastwood, P. R.; Gonzalez Rodriguez, J.; Giulio Matassa, V. New substituted indolin-2-one derivatives and their use as p39 mitogen-activated kinase inhibitors. U.S. Patent WO2009132774A1.

${ }^{29}$ Wunderlich, S. H.; Knochel, P. Preparation of Functionalized Aryl Iron(II) Compounds and a Nickel-Catalyzed CrossCoupling with Alkyl Halides. Angew. Chem. Int. Ed. 2009, 48 (51), 9717-9720.

${ }^{30}$ Wunderlich, S.; Bresser, T.; Dunst, C.; Monzon, G.; Knochel, P. Efficient Preparation of Polyfunctional Organometallics via Directed Ortho-Metalation. Synthesis 2010, 2010 (15), 2670-2678.

${ }^{31}$ Wunderlich, S. H.; Knochel, P. Atom-Economical Preparation of Aryl- and Heteroaryl-Lanthanum Reagents by Directed Ortho -Metalation by Using Tmp 3 [La]. Chem. - Eur. J. 2010, 16 (11), 3304-3307.

${ }^{32}$ Owton, W. M. Synthesis of Substituted 3-Trifluoromethylbenzo[b]Thiophenes. Tetrahedron Lett. 2003, 44 (38), 7147-7149.

33 Weidmann, N.; Ketels, M.; Knochel, P. Sodiation of Arenes and Heteroarenes in Continuous Flow. Angew. Chem. Int. Ed. 2018, 57 (33), 10748-10751.

${ }^{34}$ Maddock, L. C. H.; Nixon, T.; Kennedy, A. R.; Probert, M. R.; Clegg, W.; Hevia, E. Utilising Sodium-Mediated Ferration for Regioselective Functionalisation of Fluoroarenes via $\mathrm{C}-\mathrm{H}$ and $\mathrm{C}-\mathrm{F}$ Bond Activations. Angew. Chem. Int. Ed. 2018, 57 (1), 187191. 


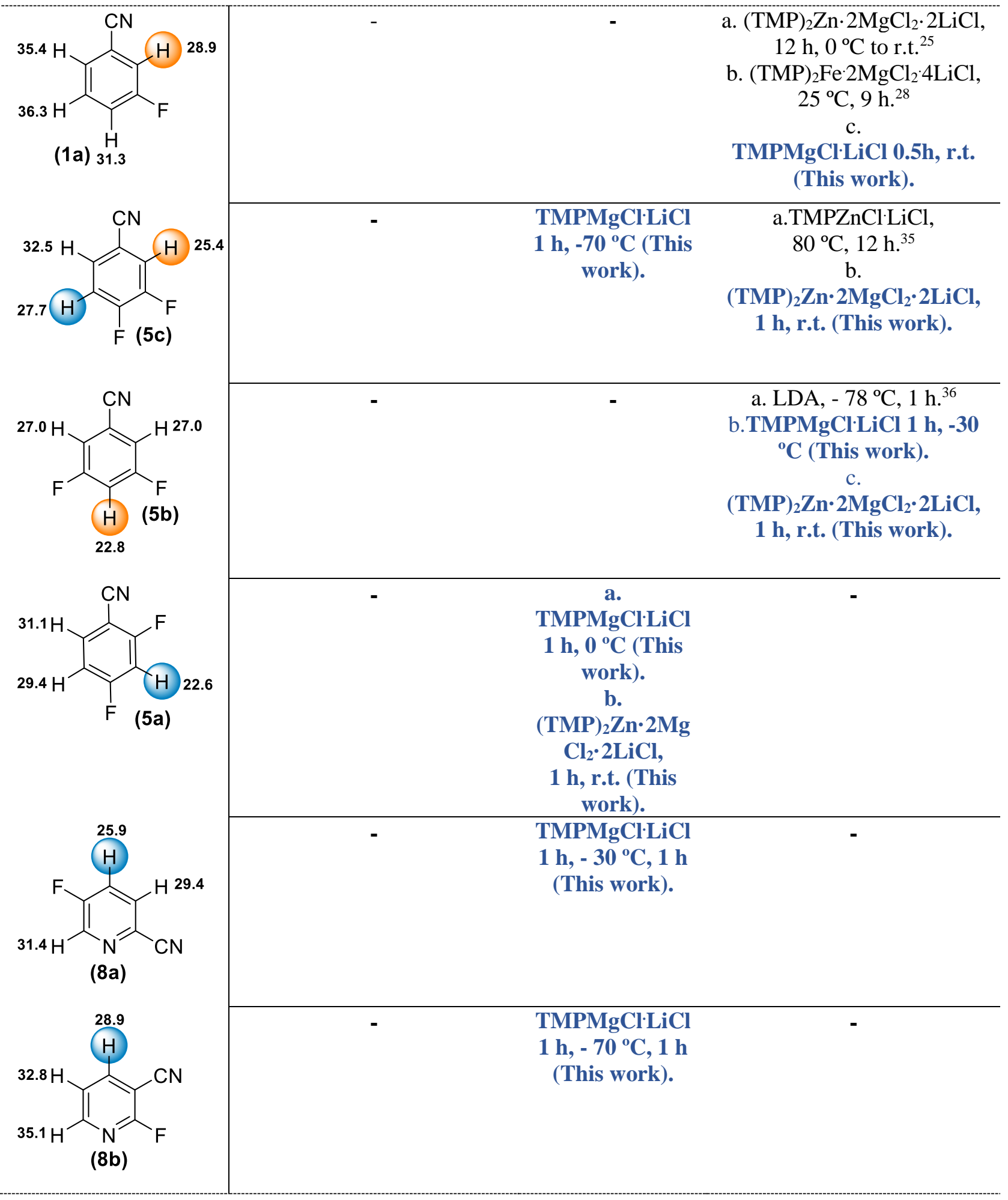

35 Otsuka, S.; Yorimitsu, H.; Osuka, A. Palladium-Catalyzed Zinc-Amide-Mediated C-H Arylation of Fluoroarenes and Heteroarenes with Aryl Sulfides. Chem. - Eur. J. 2015, 21 (42), 14703-14707.

${ }^{36}$ Huang, Y.; Cai, Z.; Li, S.; Nabulsi, N. Carson, R. Radiolabeled pharmaceuticals and methods of making and using same. U.S. Patent WO 2018/152339 A1. 


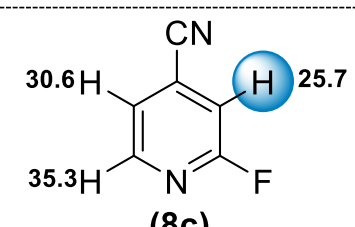

(8c)
TMPMgCl·LiCl

$1 \mathrm{~h},-\mathbf{7 0}^{\circ} \mathrm{C}$ or

$(\mathrm{TMP})_{2} \mathrm{Zn} \cdot 2 \mathrm{Mg}$

$\mathrm{Cl}_{2} \cdot 2 \mathrm{LiCl}$, r.t., 1

h (This work).

"Metalation details: green refers to the literature, blue to this work and orange to both of them if the same site was explored.

When compared to the benzonitrile, the fluoro group significantly influences the decrease in $\mathrm{p} K_{\mathrm{a}}$ for the aromatic hydrogens from the studied substrates as shown by DFT calculations (Table $\mathrm{S} 2$ ). Except for $\mathbf{5 c}$ where only $(\mathrm{TMP})_{2} \mathrm{Zn} \cdot 2 \mathrm{MgCl}_{2} \cdot 2 \mathrm{LiCl}$ showed preference upon the most acidic aromatic hydrogen, $\mathrm{TMPMgCl} \cdot \mathrm{LiCl}$ predominantly abstracted the most acidic hydrogens for the other nitriles, which are located ortho to the fluoro group in most of the cases. The latter base is prone to undergo kinetic driven metalations ${ }^{37}$ instead of the thermodynamically based ones as observed for the TMP-zinc base TMPZnCl $\mathrm{LiCl}^{38}$ The thermodynamic control for the deprotonation at $\mathrm{C} 2$ in $\mathbf{5 c}$ with $(\mathrm{TMP})_{2} \mathrm{Zn} \cdot 2 \mathrm{MgCl}_{2} \cdot 2 \mathrm{LiCl}$ can benefit from the electron withdrawing effect by the adjacent groups (-CN and $-\mathrm{F})$ and its influence in acidity and stabilization of the derived anion as observed by Osuka and coworkers for the same substrate via TMPZnCl LiCl. ${ }^{35}$ Interesting, despite the coordinating capability of nitrogen from the studied pyridines $(\mathbf{8 a}-\mathbf{c})$ to the metal center in $\mathrm{TMPMgCl} \mathrm{LiCl}^{39}$, the inductive effect stablished by fluoro and cyano groups governed the regioselectivity for the magnesiation sites.

\footnotetext{
37 Kremsmair, A.; Harenberg, J. H.; Schwärzer, K.; Hess, A.; Knochel, P. Preparation and Reactions of Polyfunctional Magnesium and Zinc Organometallics in Organic Synthesis. Chem. Sci. 2021, 12 (17), 6011-6019.

38 Balkenhohl, M.; Jangra, H.; Makarov, I. S.; Yang, S.; Zipse, H.; Knochel, P. A Predictive Model Towards Site-Selective Metalations of Functionalized Heterocycles, Arenes, Olefins, and Alkanes Using TMPZnCl·LiCl. Angew. Chem. Int. Ed. 2020, 59 (35), 14992-14999.

${ }^{39}$ Balkenhohl, M.; Knochel, P. Regioselective C-H Activation of Substituted Pyridines and Other Azines Using Mg- and ZnTMP-Bases. SynOpen 2018, 02 (01), 0078-0095.
} 


\section{NMR SPECTRA}
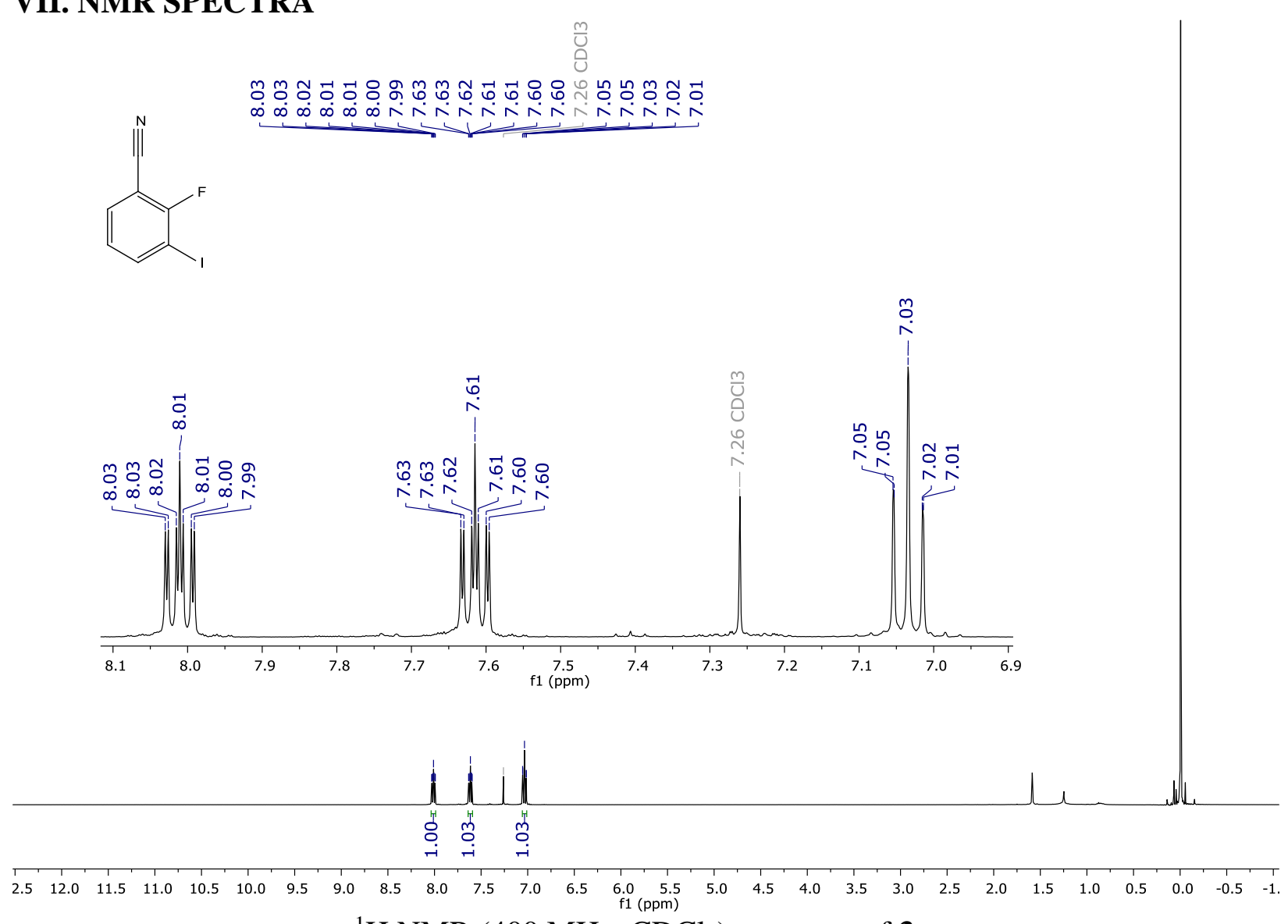

${ }^{1} \mathrm{H}$ NMR (400 MHz, $\mathrm{CDCl}_{3}$ ) spectrum of 3a.
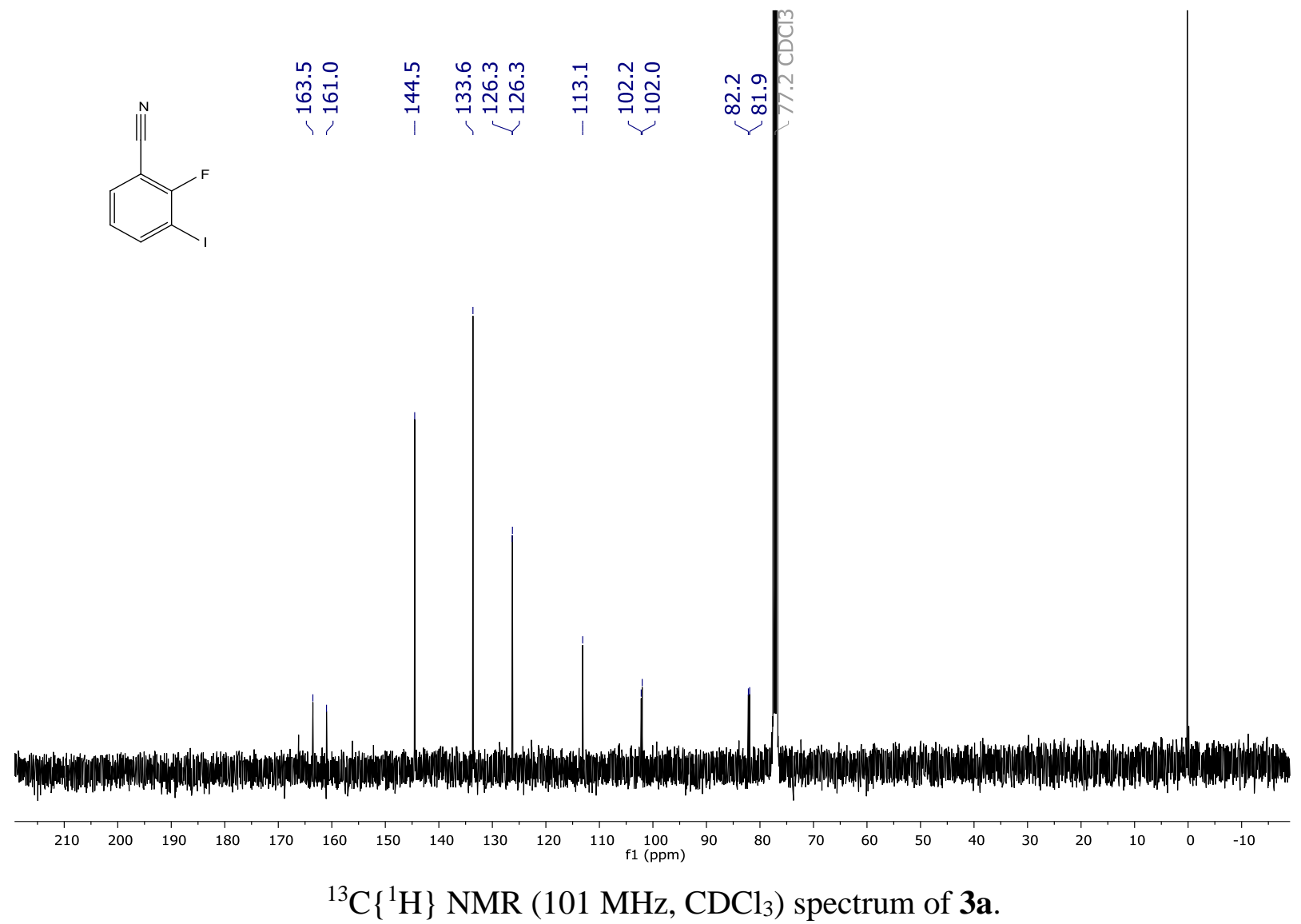

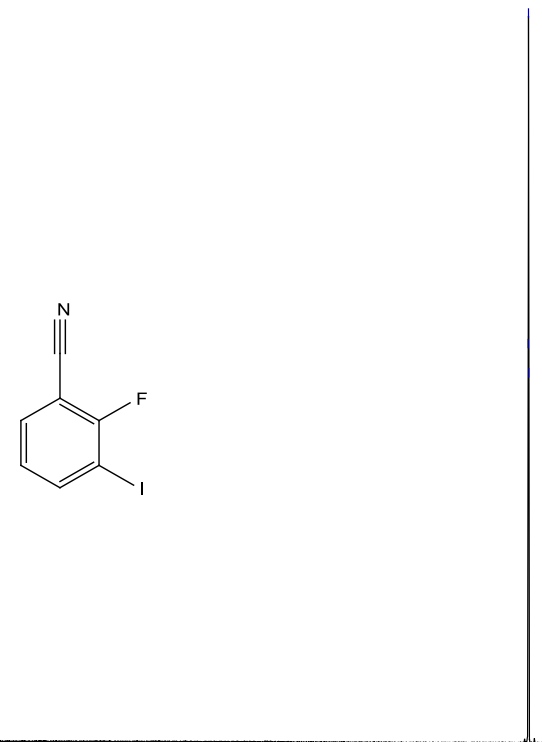

\begin{tabular}{llllllllllllllllllllllllllllllllllllllllllllllll}
\hline 0 & -5 & -10 & -15 & -20 & -25 & -30 & -35 & -40 & -45 & -50 & -55 & -60 & -65 & -70 & -75 & -80 & -85 & -90 & -95 & -100 & -105 & -110 & -115 & -120 & -125 & -130 & -135 & -140 & -145 & -1
\end{tabular}

${ }^{19} \mathrm{~F}$ NMR (282 MHz, $\left.\mathrm{CDCl}_{3}\right)$ spectrum of 3a.

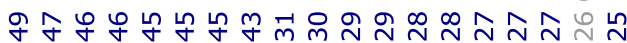

บ

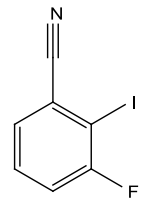

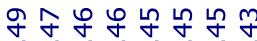

ก
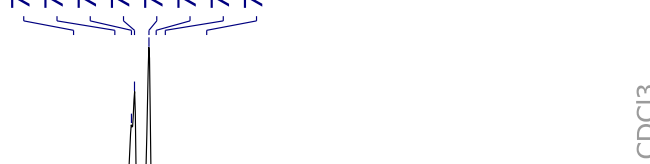

$\overline{\mathrm{U}}$

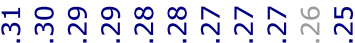
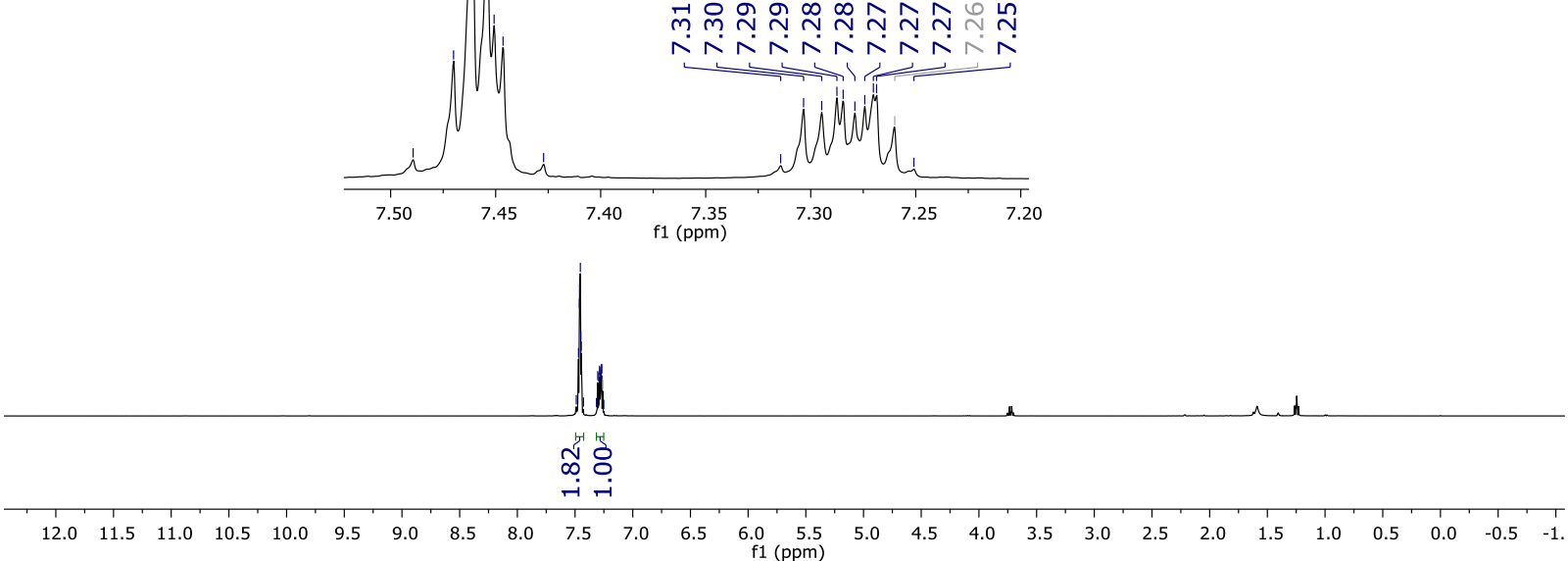

${ }^{1} \mathrm{H}$ NMR (400 MHz, $\mathrm{CDCl}_{3}$ ) spectrum of $\mathbf{3 b}$. 


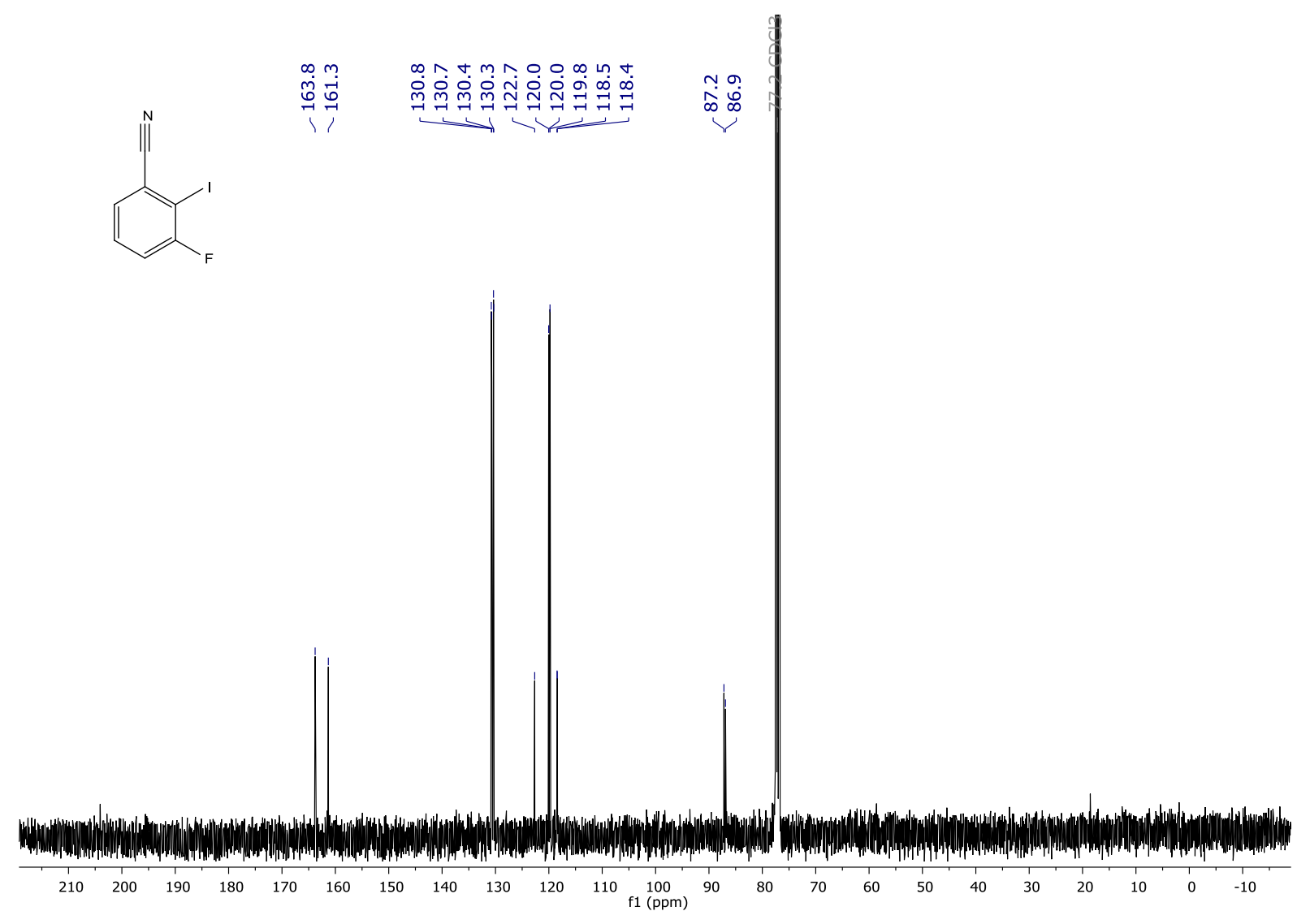

${ }^{13} \mathrm{C}\left\{{ }^{1} \mathrm{H}\right\}$ NMR $\left(101 \mathrm{MHz}, \mathrm{CDCl}_{3}\right)$ spectrum of $\mathbf{3 b}$.

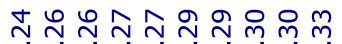

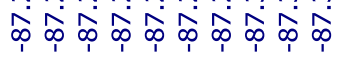

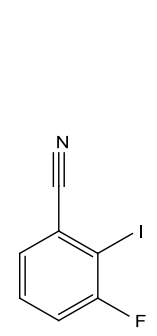

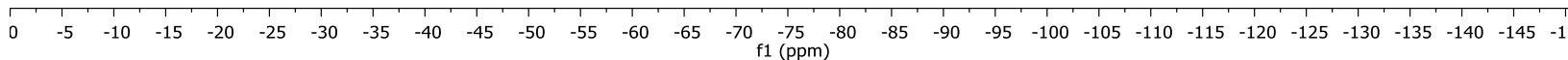

${ }^{19} \mathrm{~F}$ NMR (282 MHz, $\mathrm{CDCl}_{3}$ ) spectrum of $\mathbf{3 b}$. 


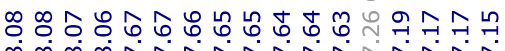

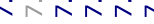
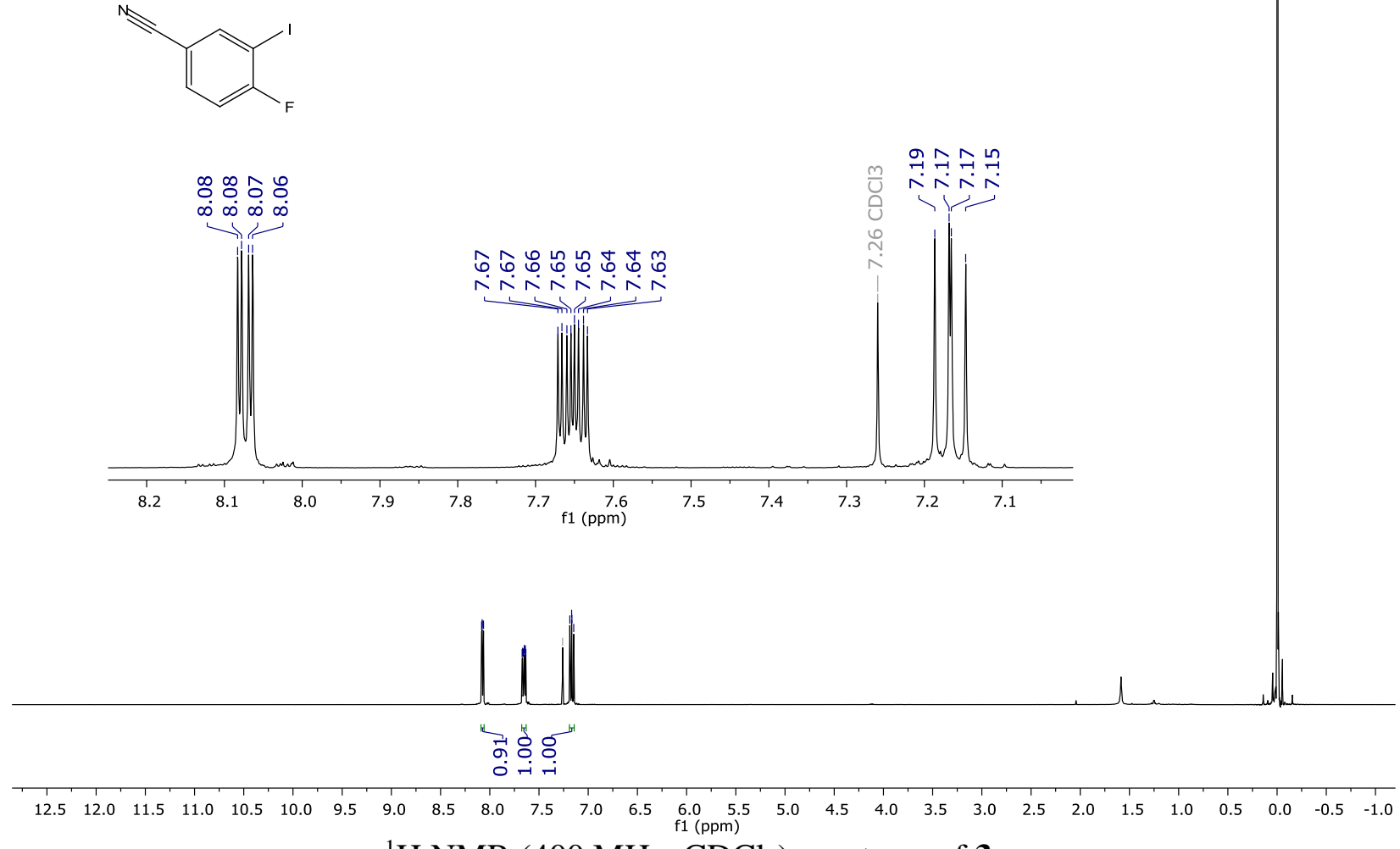

${ }^{1} \mathrm{H} \mathrm{NMR}\left(400 \mathrm{MHz}, \mathrm{CDCl}_{3}\right)$ spectrum of $\mathbf{3 c}$.

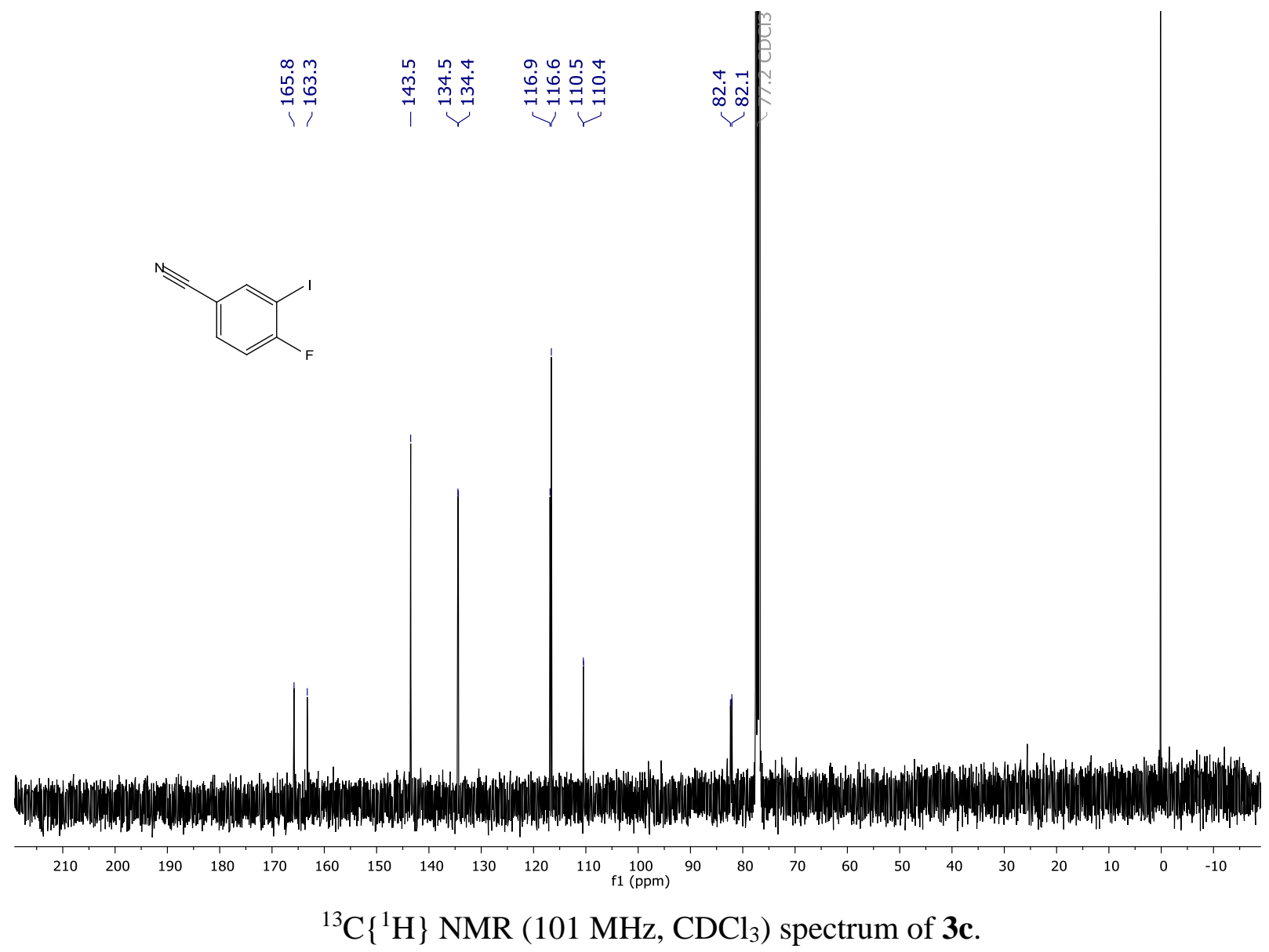



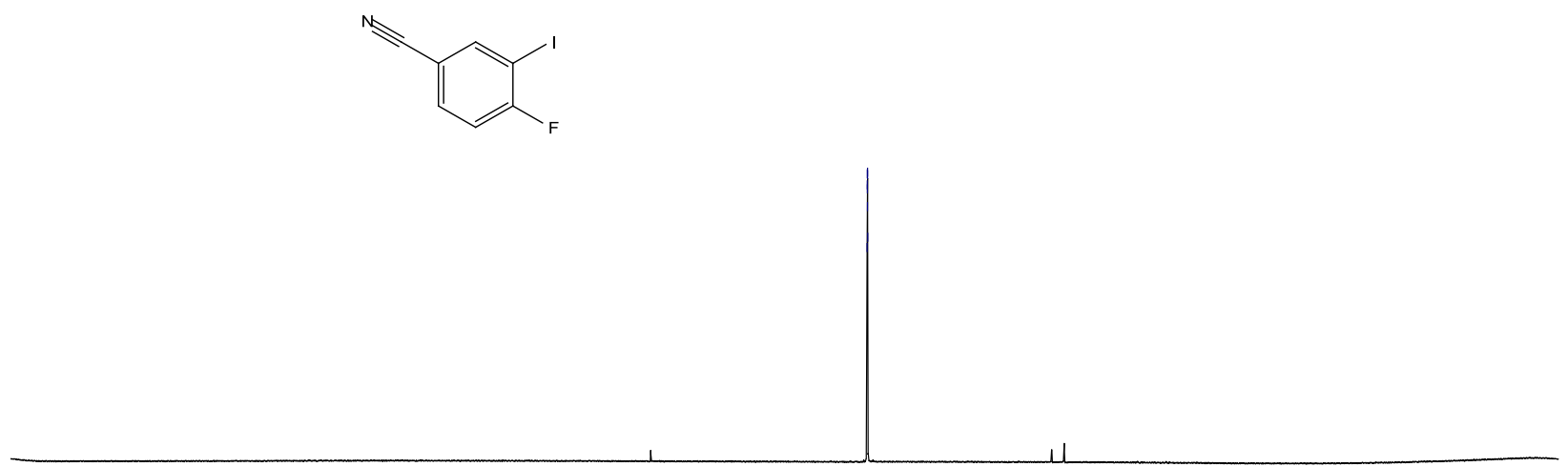

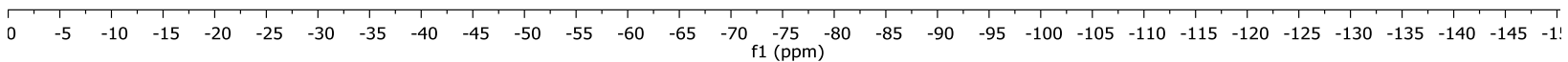

${ }^{19} \mathrm{~F} \mathrm{NMR}\left(282 \mathrm{MHz}, \mathrm{CDCl}_{3}\right)$ spectrum of $\mathbf{3 c}$.

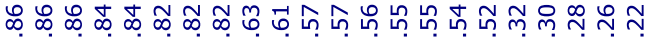

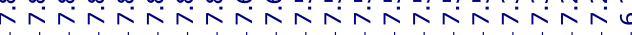

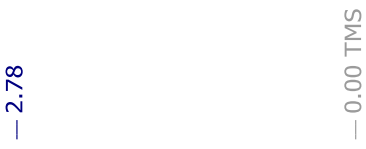
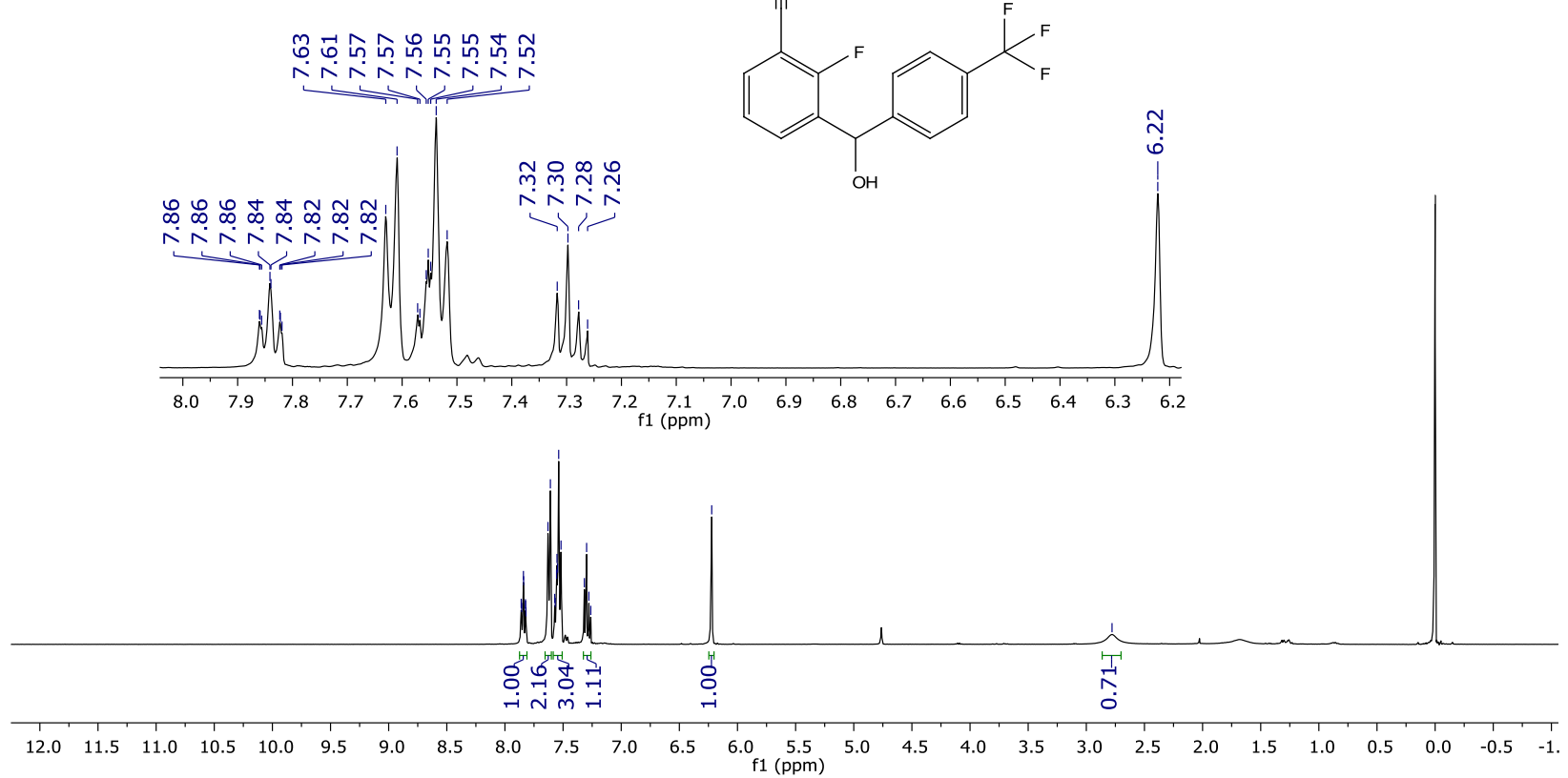

${ }^{1} \mathrm{H}$ NMR $\left(400 \mathrm{MHz}, \mathrm{CDCl}_{3}\right)$ spectrum of $\mathbf{3 d}$. 


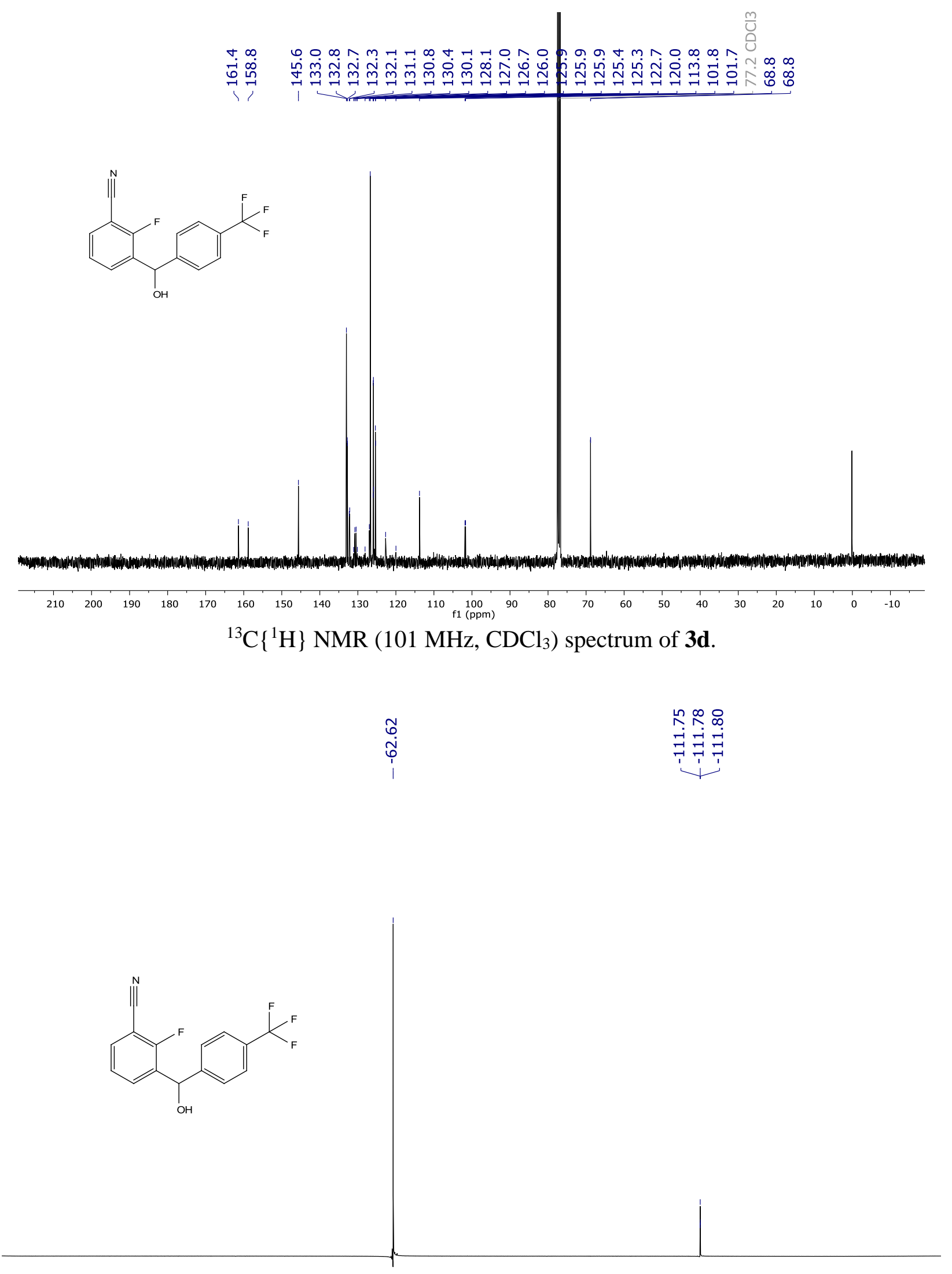

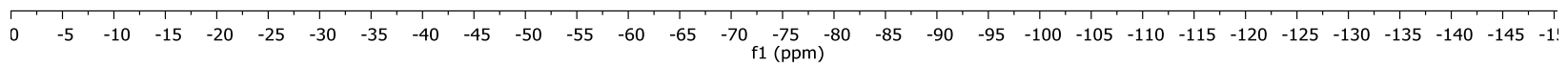

${ }^{19} \mathrm{~F}$ NMR (282 MHz, $\left.\mathrm{CDCl}_{3}\right)$ spectrum of $\mathbf{3 d}$. 
<smiles>N#Cc1cccc(C(O)c2ccccc2)c1F</smiles>
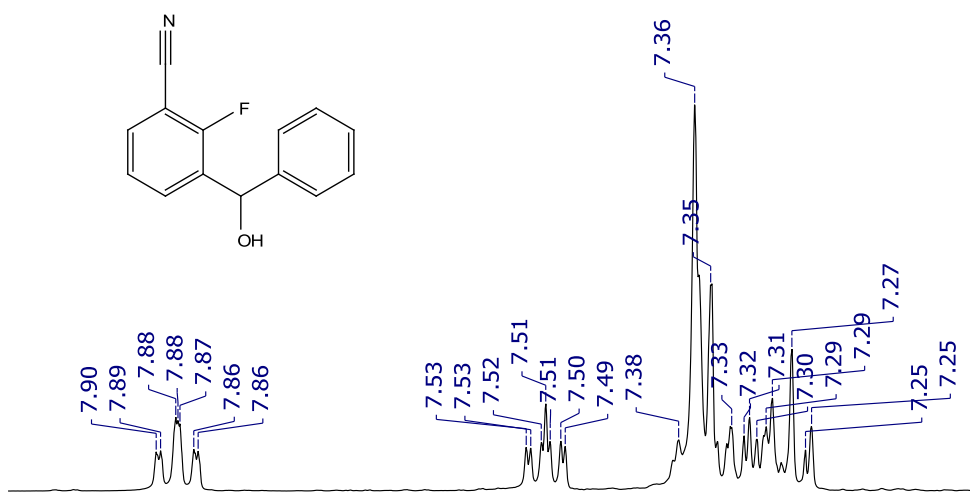

$\begin{array}{llllllllllllllllllllllllll}8.00 & 7.95 & 7.90 & 7.85 & 7.80 & 7.75 & 7.70 & 7.65 & 7.60 & 7.55 & 7.50 & 7.45 & 7.40 & 7.35 & 7.30 & 7.25 & 7.20 & 7.15 & 7.10\end{array}$

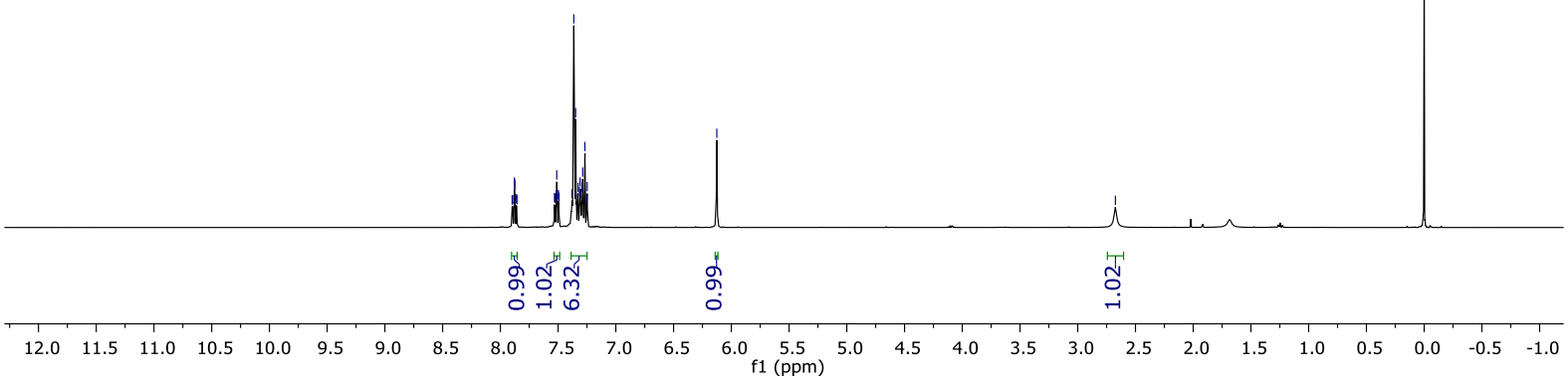

${ }^{1} \mathrm{H}$ NMR $\left(400 \mathrm{MHz}, \mathrm{CDCl}_{3}\right)$ spectrum of $\mathbf{3 e}$.

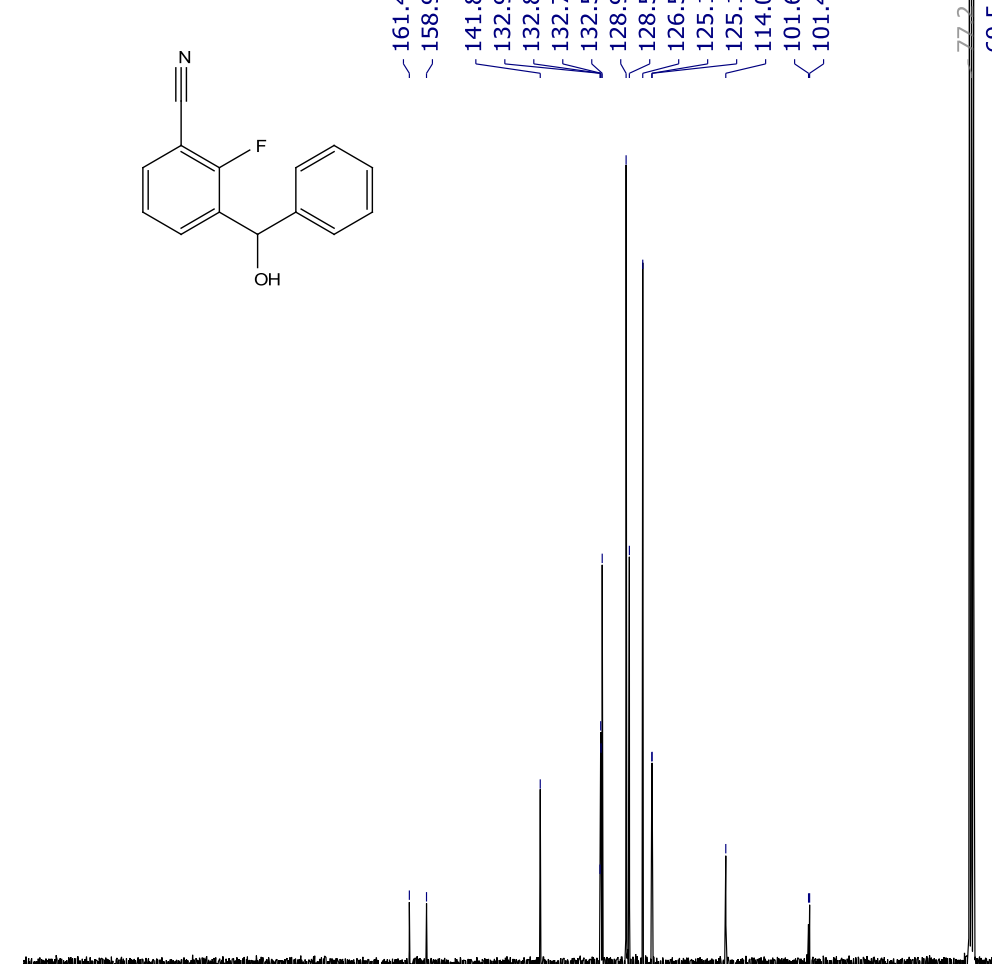

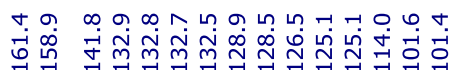



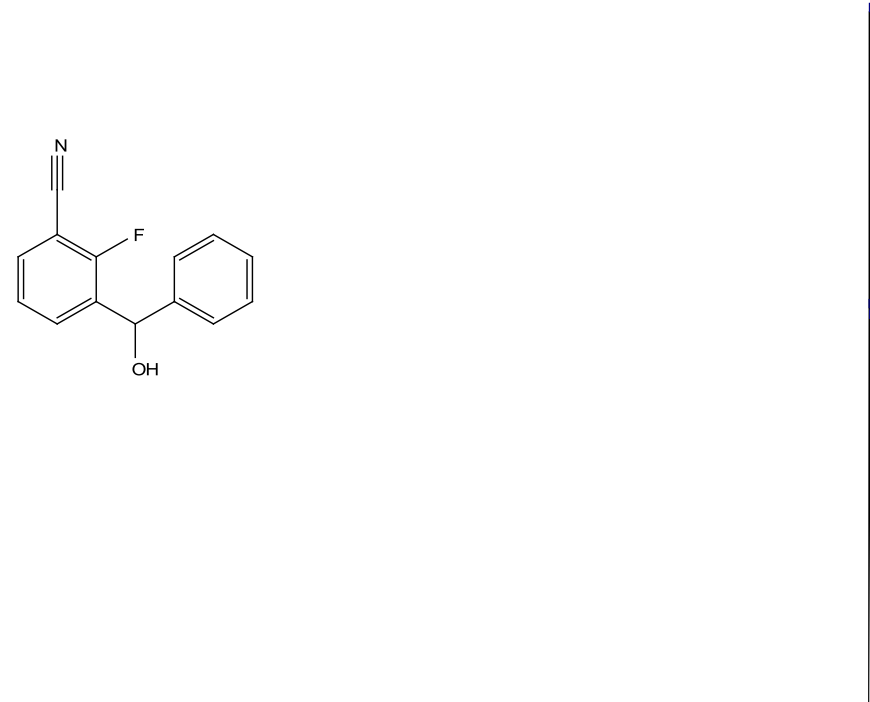

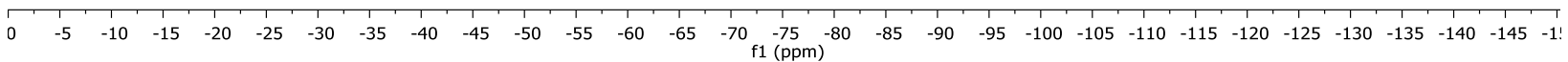

${ }^{19}$ F NMR (282 $\left.\mathrm{MHz}, \mathrm{CDCl}_{3}\right)$ spectrum of $\mathbf{3 e}$.

긍요 \&

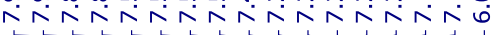
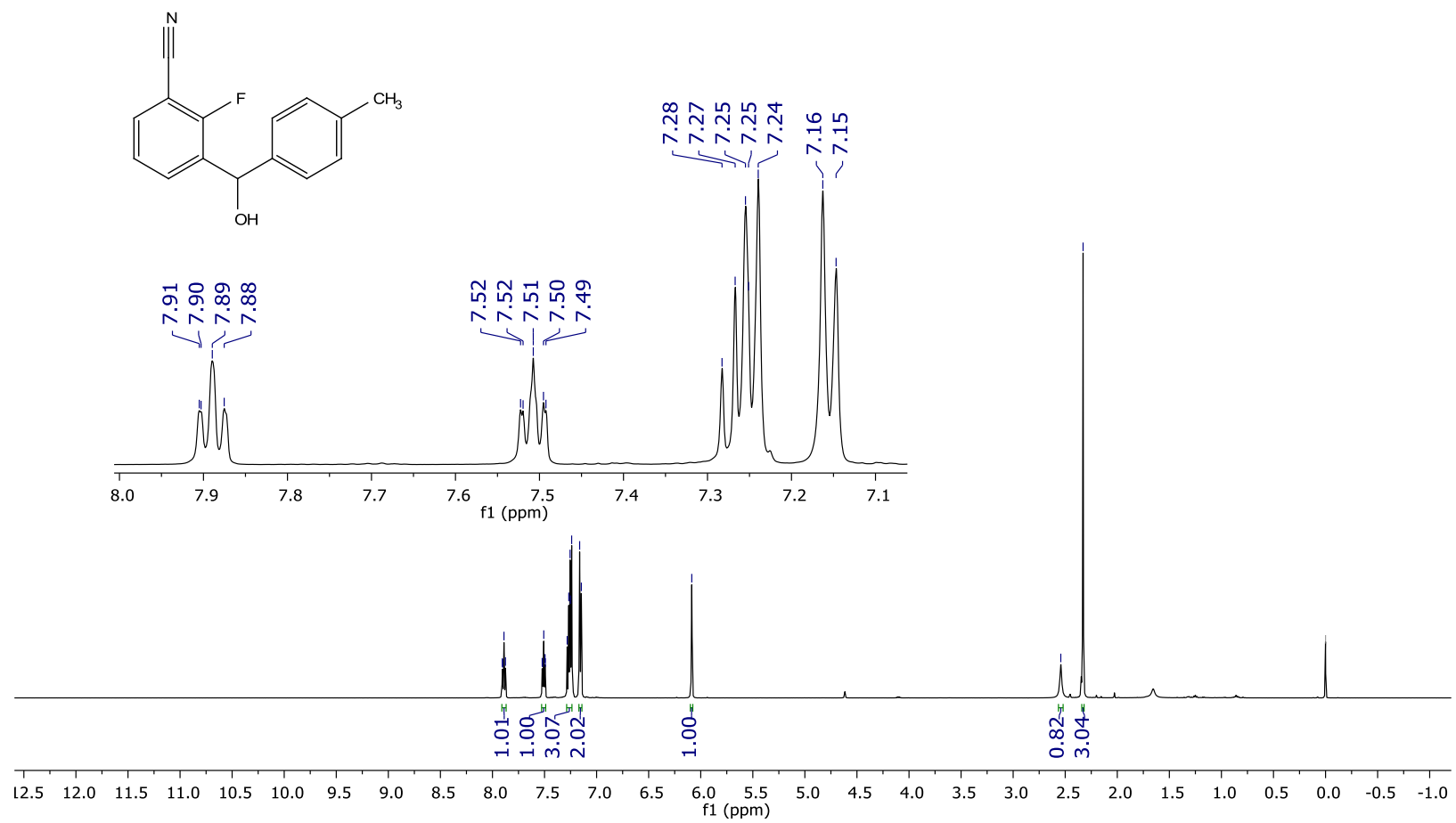

芯

$2 \pi$

$\sum_{1}^{n}$
8
0
0
1

${ }^{1} \mathrm{H}$ NMR $\left(500 \mathrm{MHz}, \mathrm{CDCl}_{3}\right)$ spectrum of $\mathbf{3 f}$. 


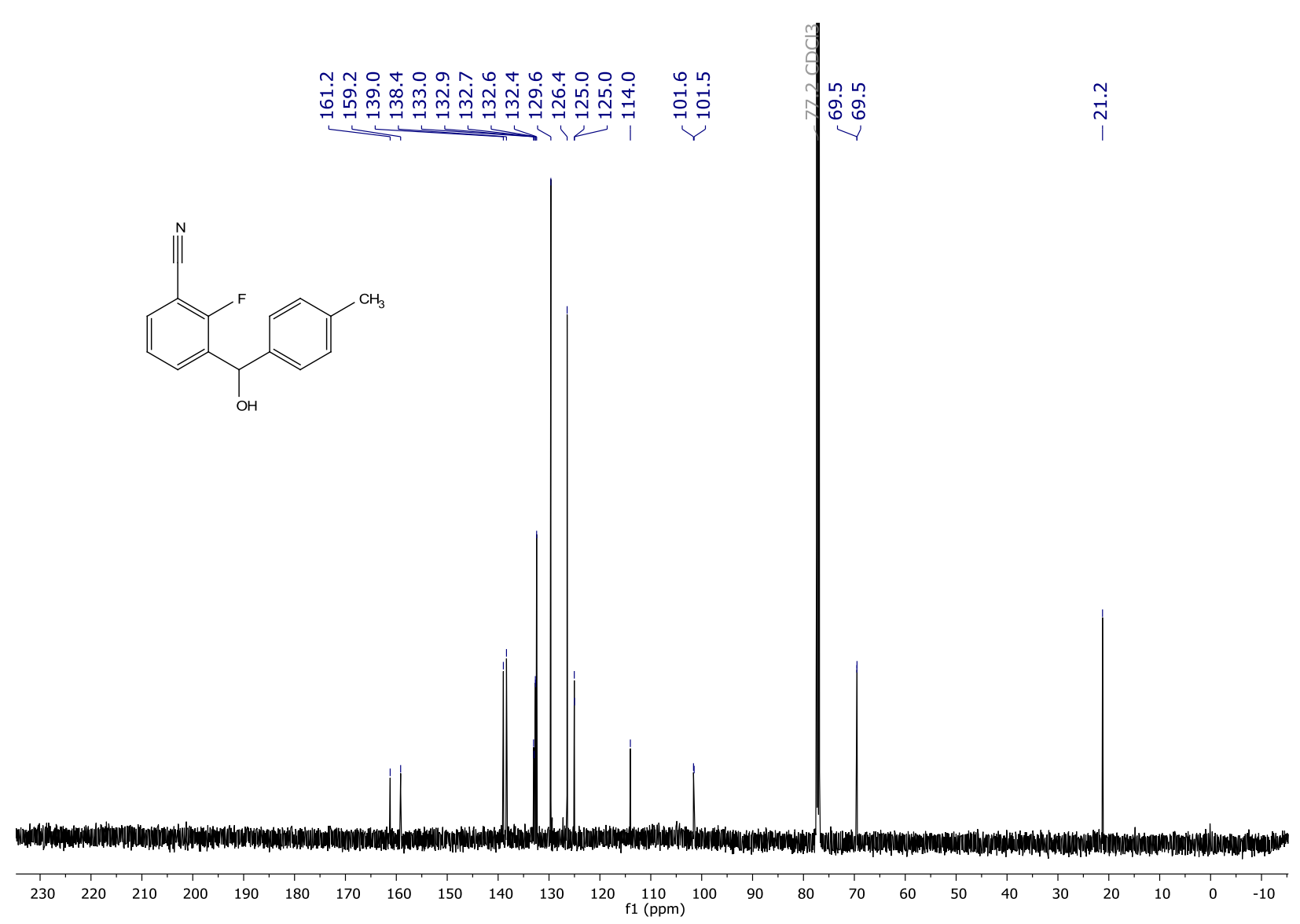

${ }^{13} \mathrm{C}\left\{{ }^{1} \mathrm{H}\right\}$ NMR (126 MHz, $\left.\mathrm{CDCl}_{3}\right)$ spectrum of $\mathbf{3 f}$.

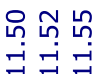

$\exists$

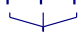

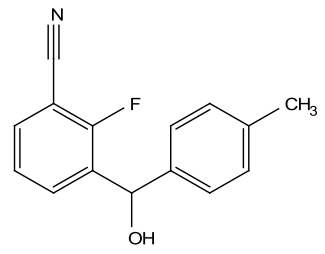

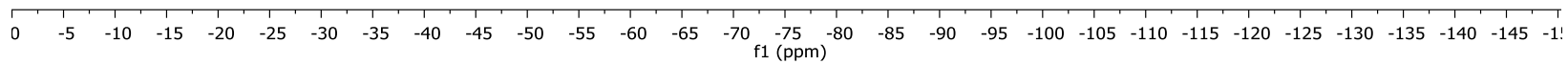

${ }^{19} \mathrm{~F}$ NMR $\left(282 \mathrm{MHz}, \mathrm{CDCl}_{3}\right)$ spectrum of $\mathbf{3 f}$. 
<smiles>N#Cc1cccc(C(O)c2ccco2)c1F</smiles>
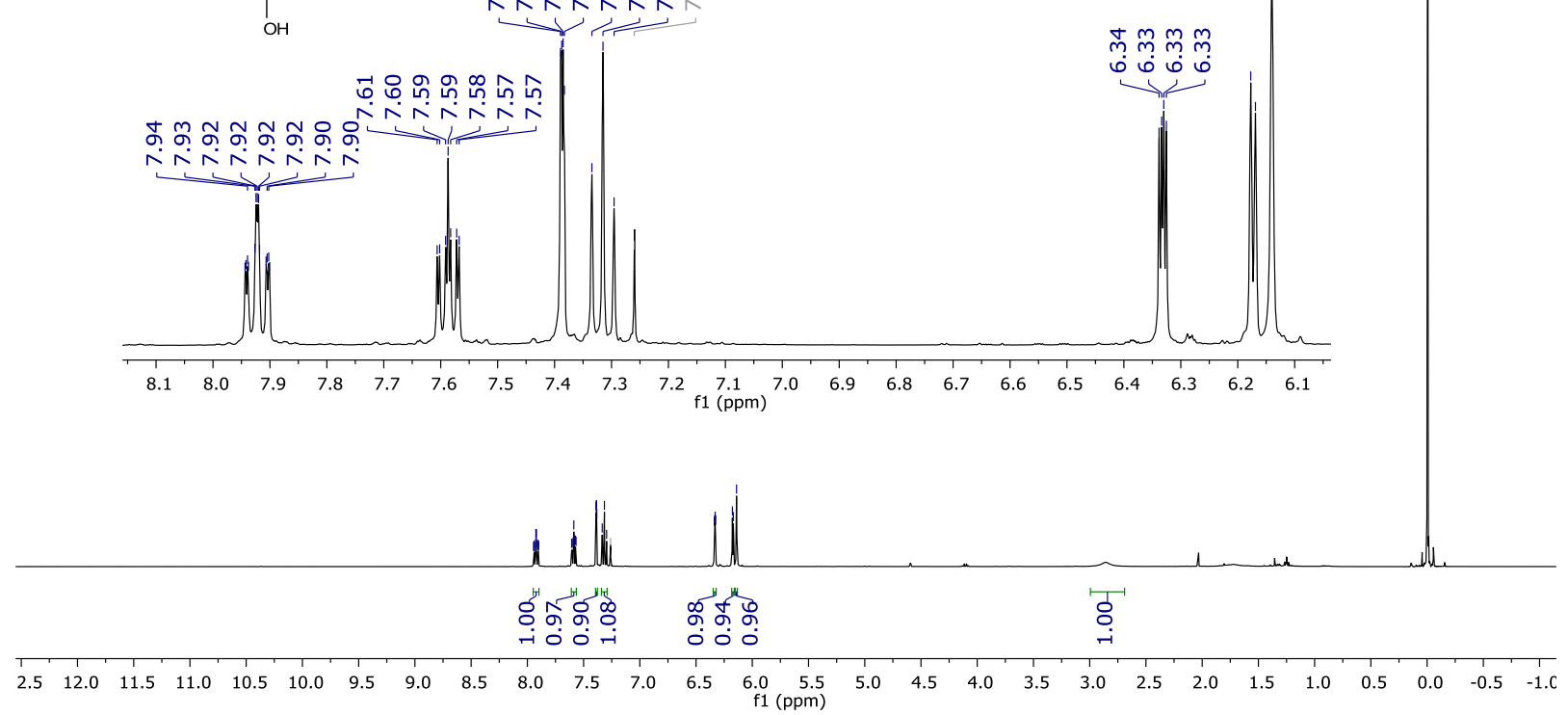

${ }^{1} \mathrm{H}$ NMR (400 MHz, $\mathrm{CDCl}_{3}$ ) spectrum of $\mathbf{3 g}$.

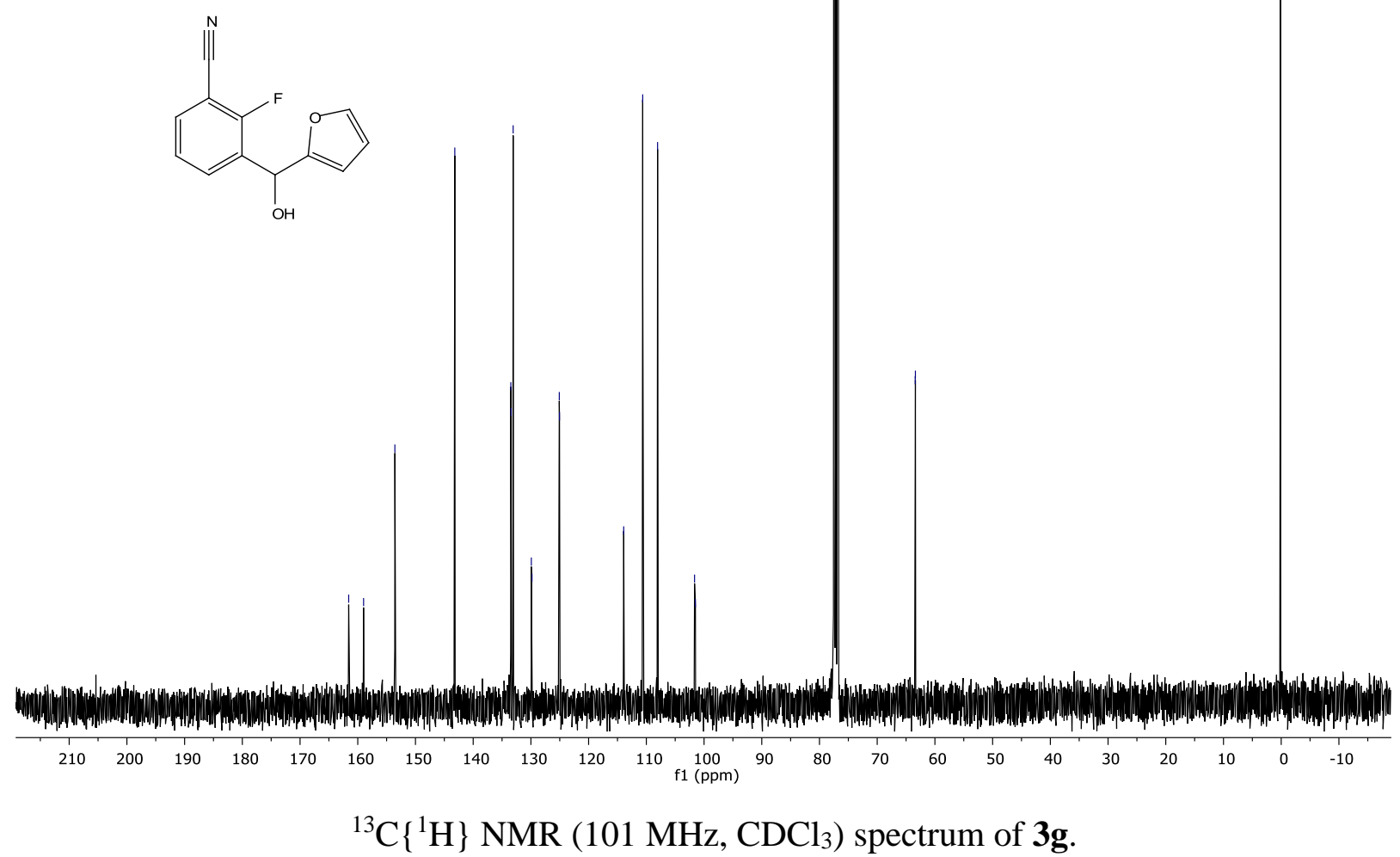

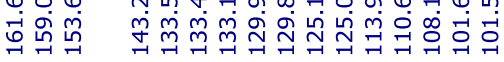

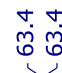



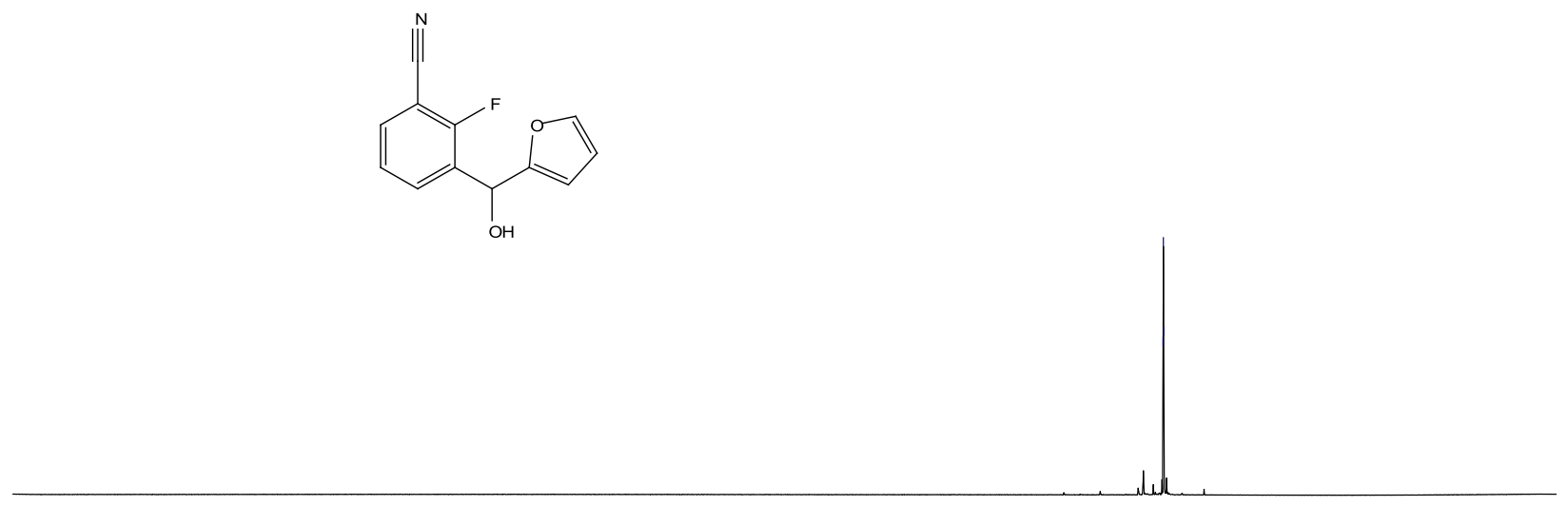

\begin{tabular}{llllllllllllllllllllllllllllllllllllll}
\hline 0 & -5 & -10 & -15 & -20 & -25 & -30 & -35 & -40 & -45 & -50 & -55 & -60 & -65 & -70 & -75 & -80 & -85 & -90 & -95 & -100 & -105 & -110 & -115 & -120 & -125 & -130 & -135 & -140 & -145 & -1
\end{tabular}

${ }^{19} \mathrm{~F}$ NMR (282 $\left.\mathrm{MHz}, \mathrm{CDCl}_{3}\right)$ spectrum of $\mathbf{3 g}$.

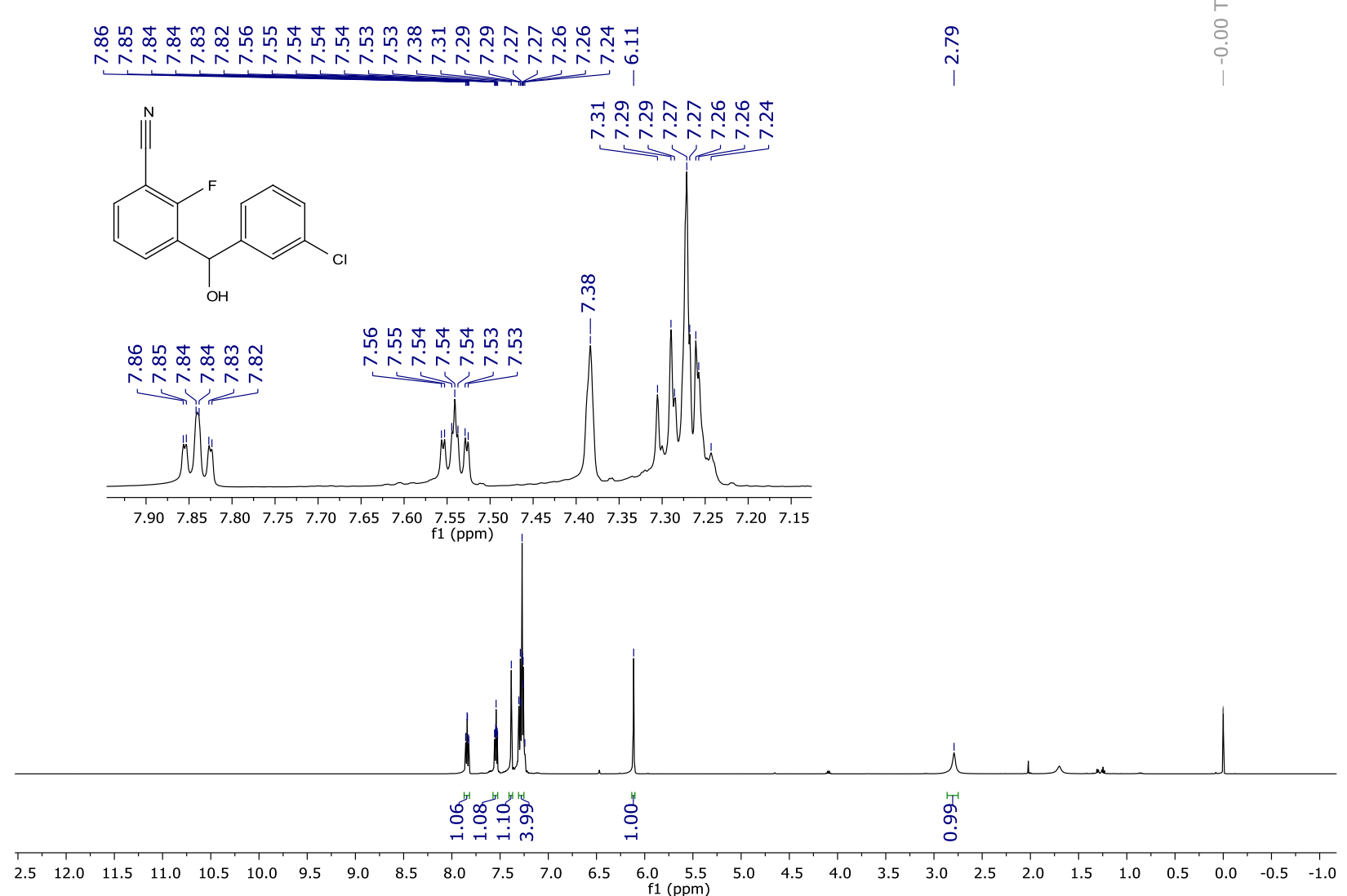

${ }^{1} \mathrm{H}$ NMR (500 MHz, $\mathrm{CDCl}_{3}$ ) spectrum of $\mathbf{3 h}$. 


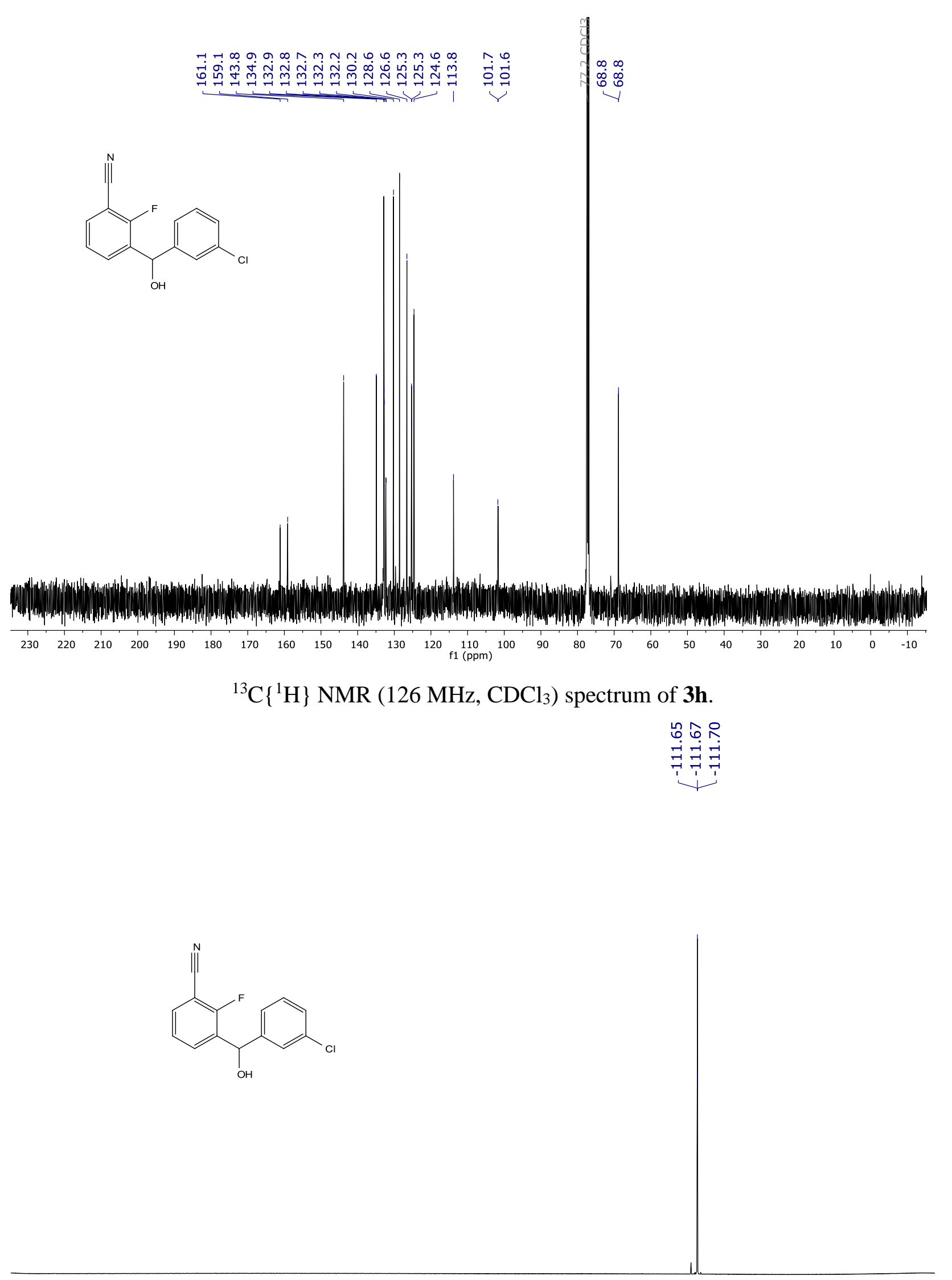

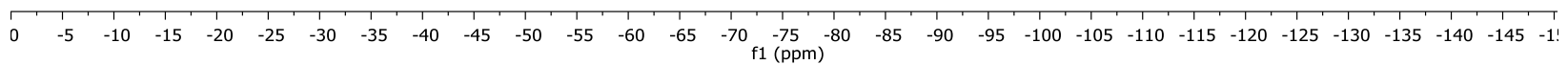

${ }^{19} \mathrm{~F}$ NMR $\left(282 \mathrm{MHz}, \mathrm{CDCl}_{3}\right)$ spectrum of $\mathbf{3 h}$. 

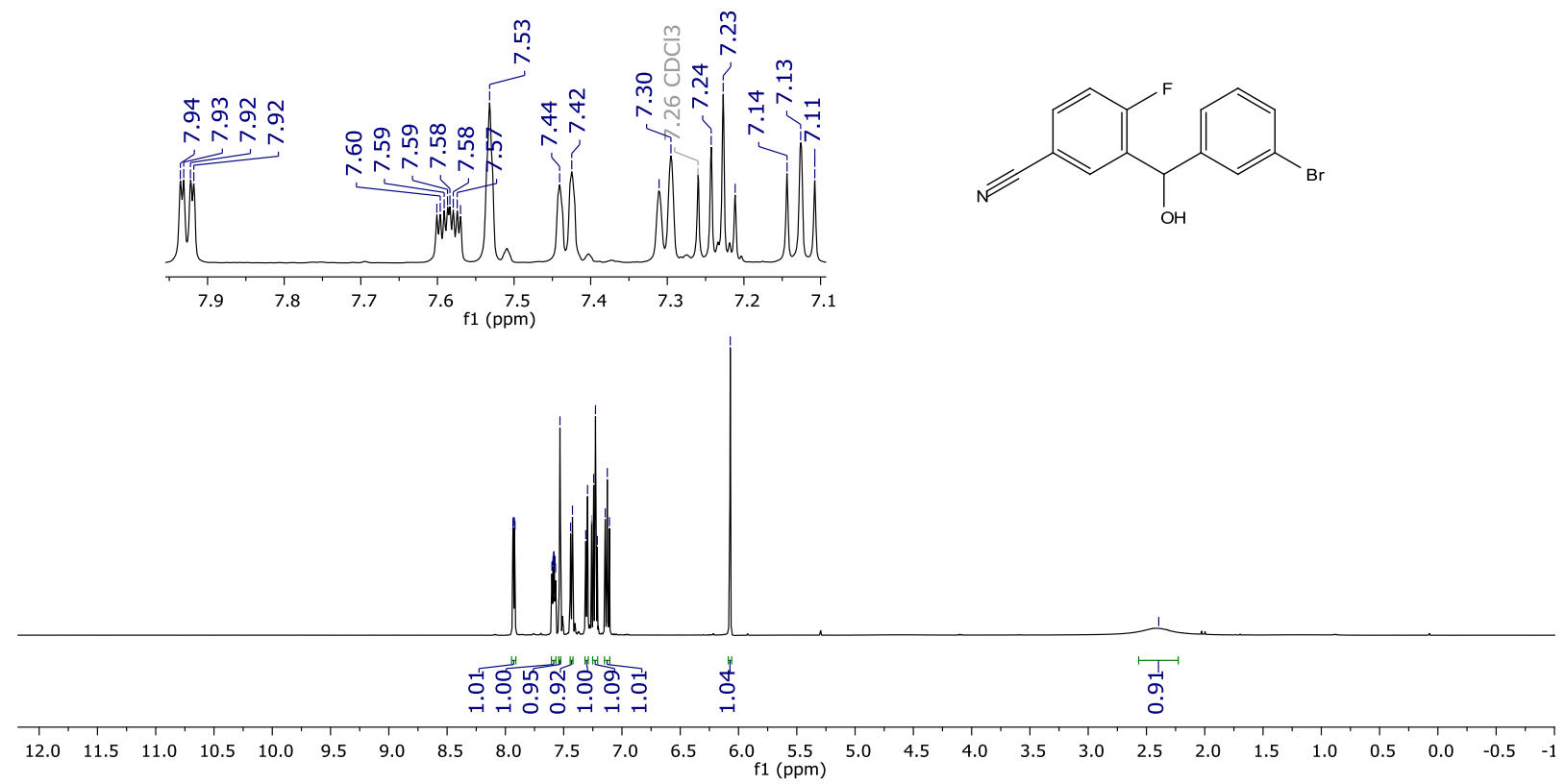

${ }^{1} \mathrm{H}$ NMR $\left(500 \mathrm{MHz}, \mathrm{CDCl}_{3}\right)$ spectrum of $3 \mathbf{3}$.

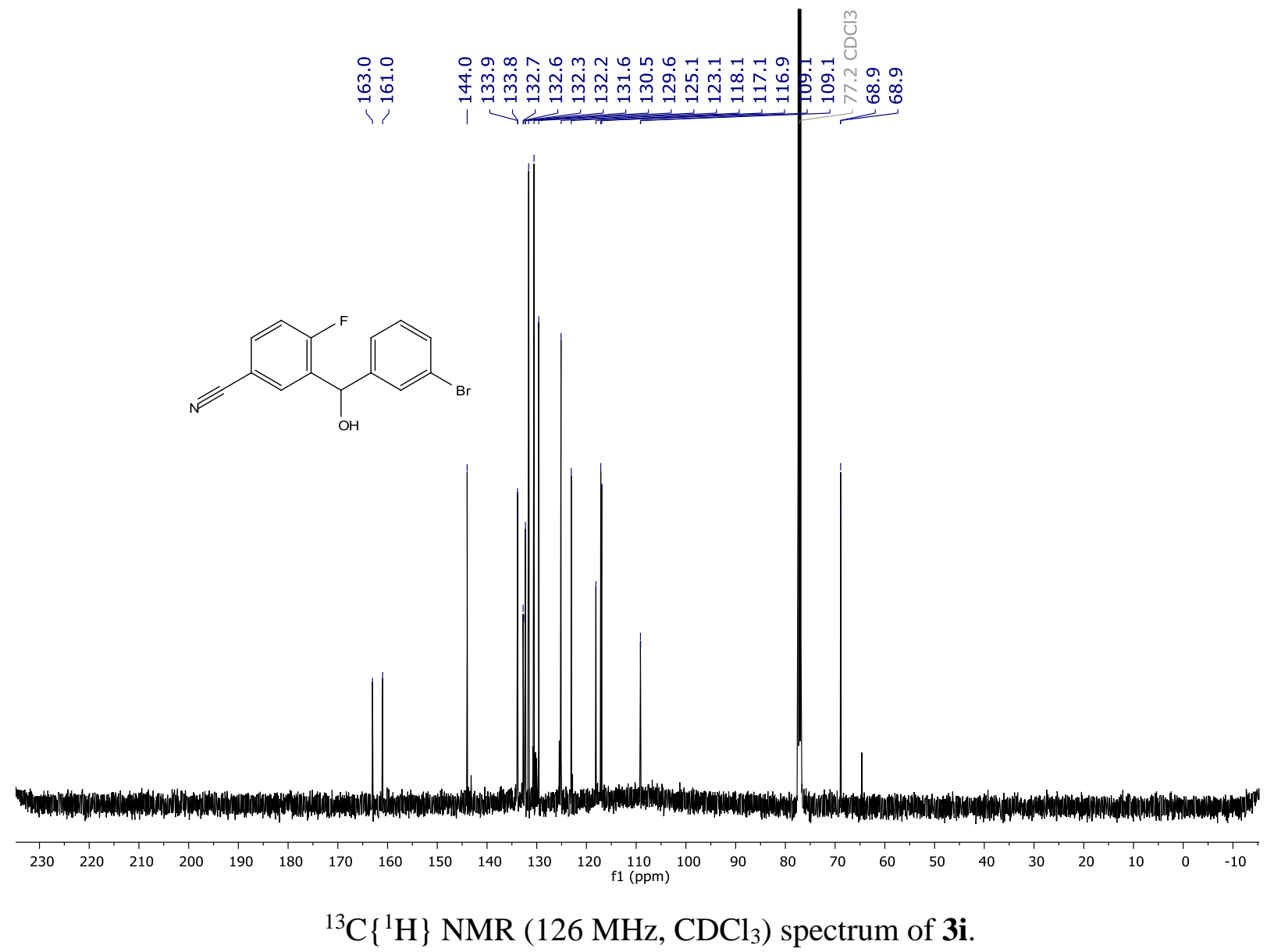




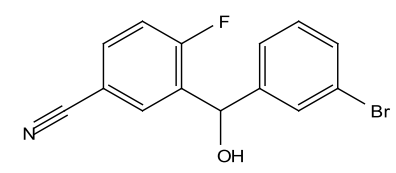

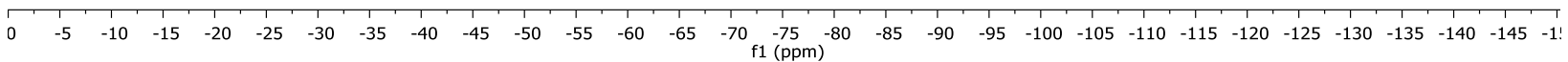

${ }^{19} \mathrm{~F}$ NMR (282 $\left.\mathrm{MHz}, \mathrm{CDCl}_{3}\right)$ spectrum of $\mathbf{3 i}$.

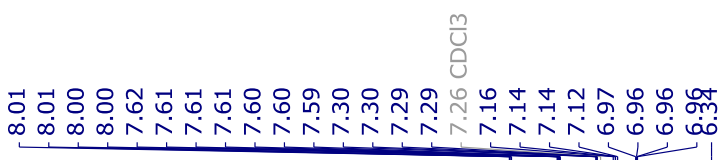

क

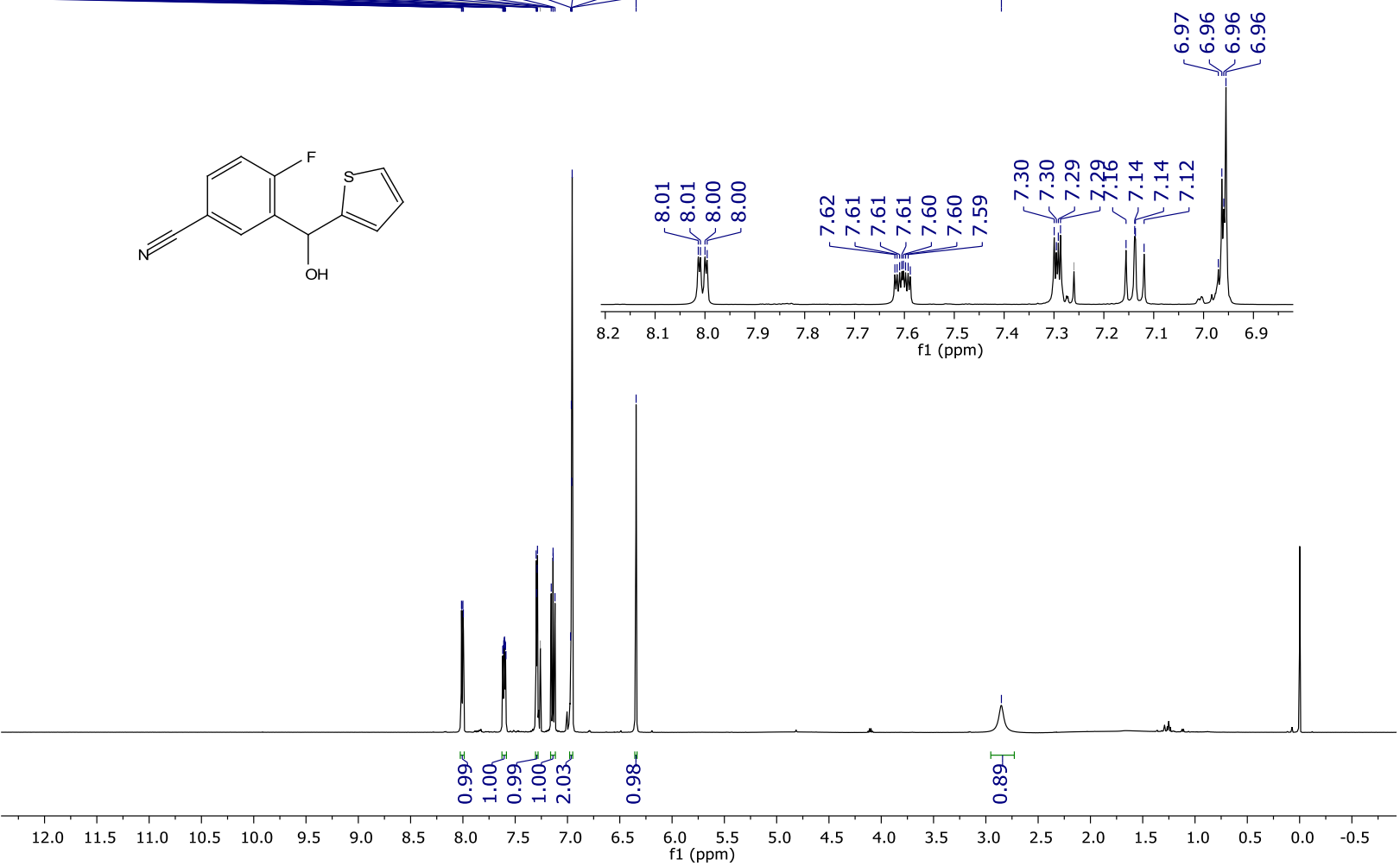

${ }^{1} \mathrm{H}$ NMR $\left(500 \mathrm{MHz}, \mathrm{CDCl}_{3}\right)$ spectrum of $\mathbf{3 j}$. 


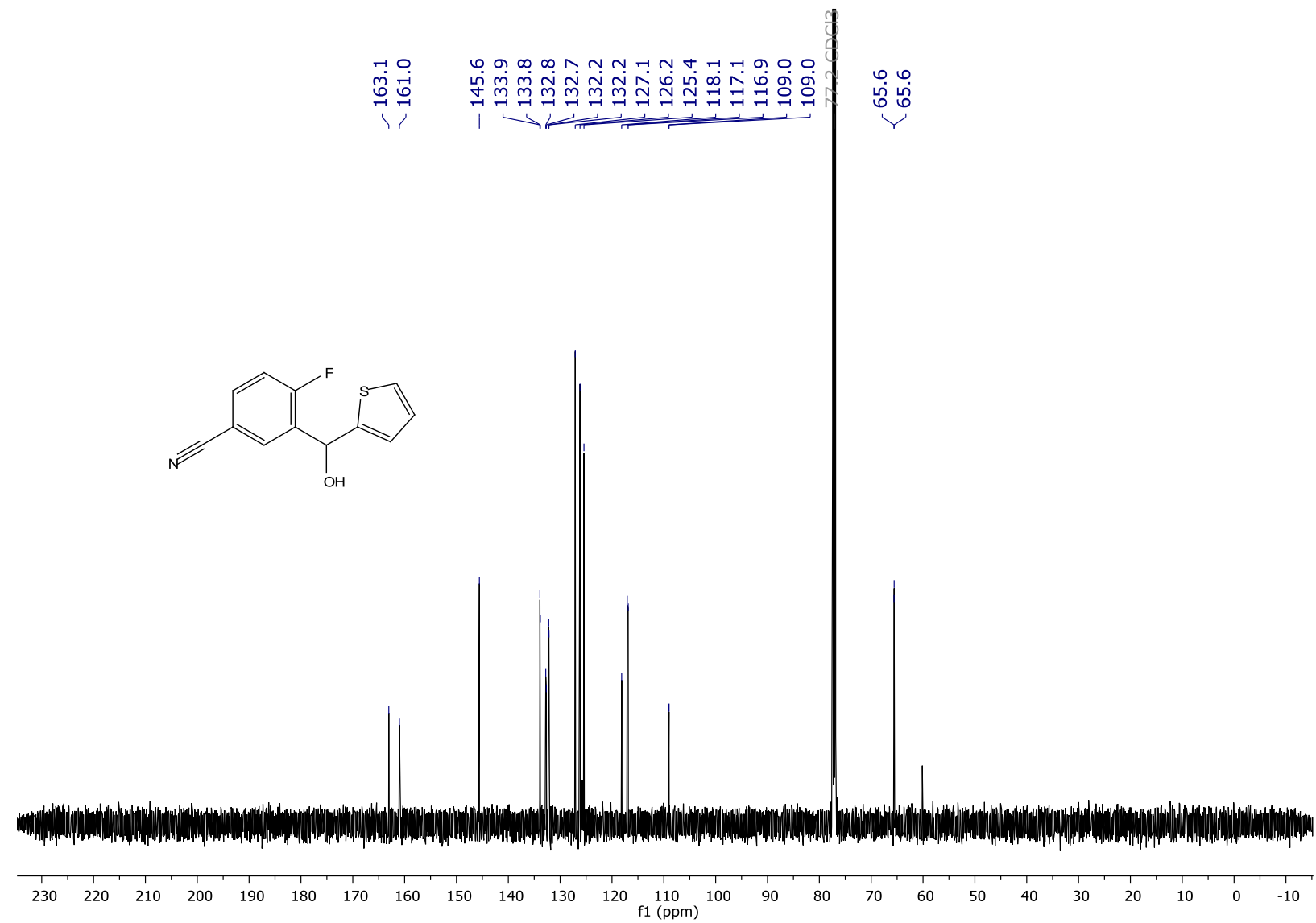

${ }^{13} \mathrm{C}\left\{{ }^{1} \mathrm{H}\right\} \mathrm{NMR}\left(126 \mathrm{MHz}, \mathrm{CDCl}_{3}\right)$ spectrum of $\mathbf{3 j}$.

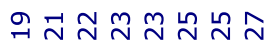

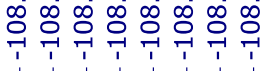
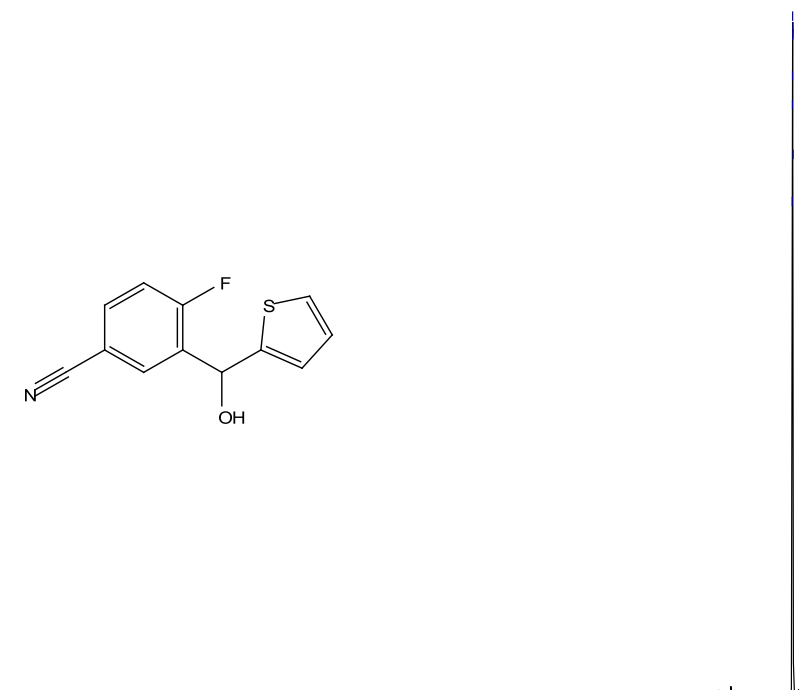

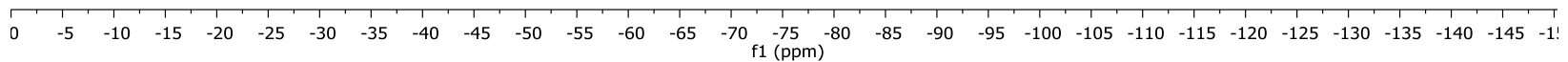

${ }^{19} \mathrm{~F}$ NMR $\left(282 \mathrm{MHz}, \mathrm{CDCl}_{3}\right)$ spectrum of $\mathbf{3 j}$. 
<smiles>N#Cc1ccc(F)c(C(O)c2ccc(F)cc2)c1</smiles>

m군

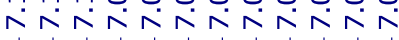
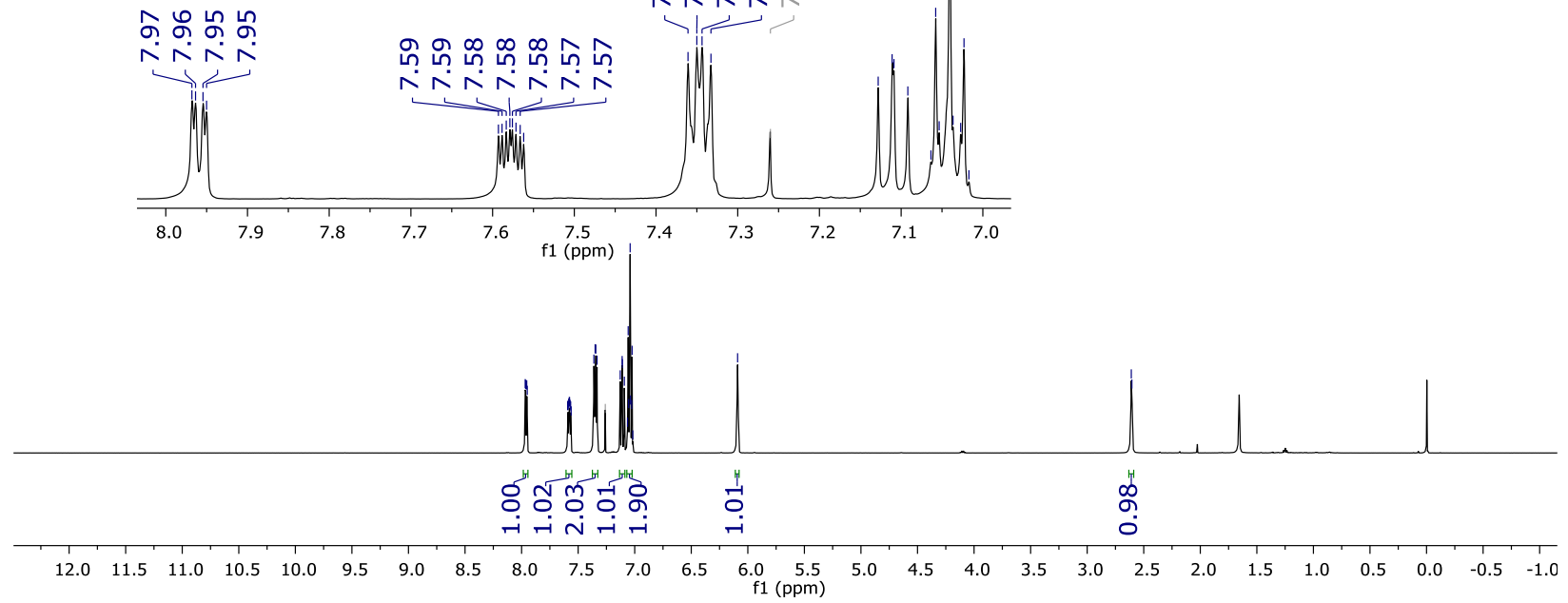

${ }^{1} \mathrm{H}$ NMR $\left(500 \mathrm{MHz}, \mathrm{CDCl}_{3}\right)$ spectrum of 3k.

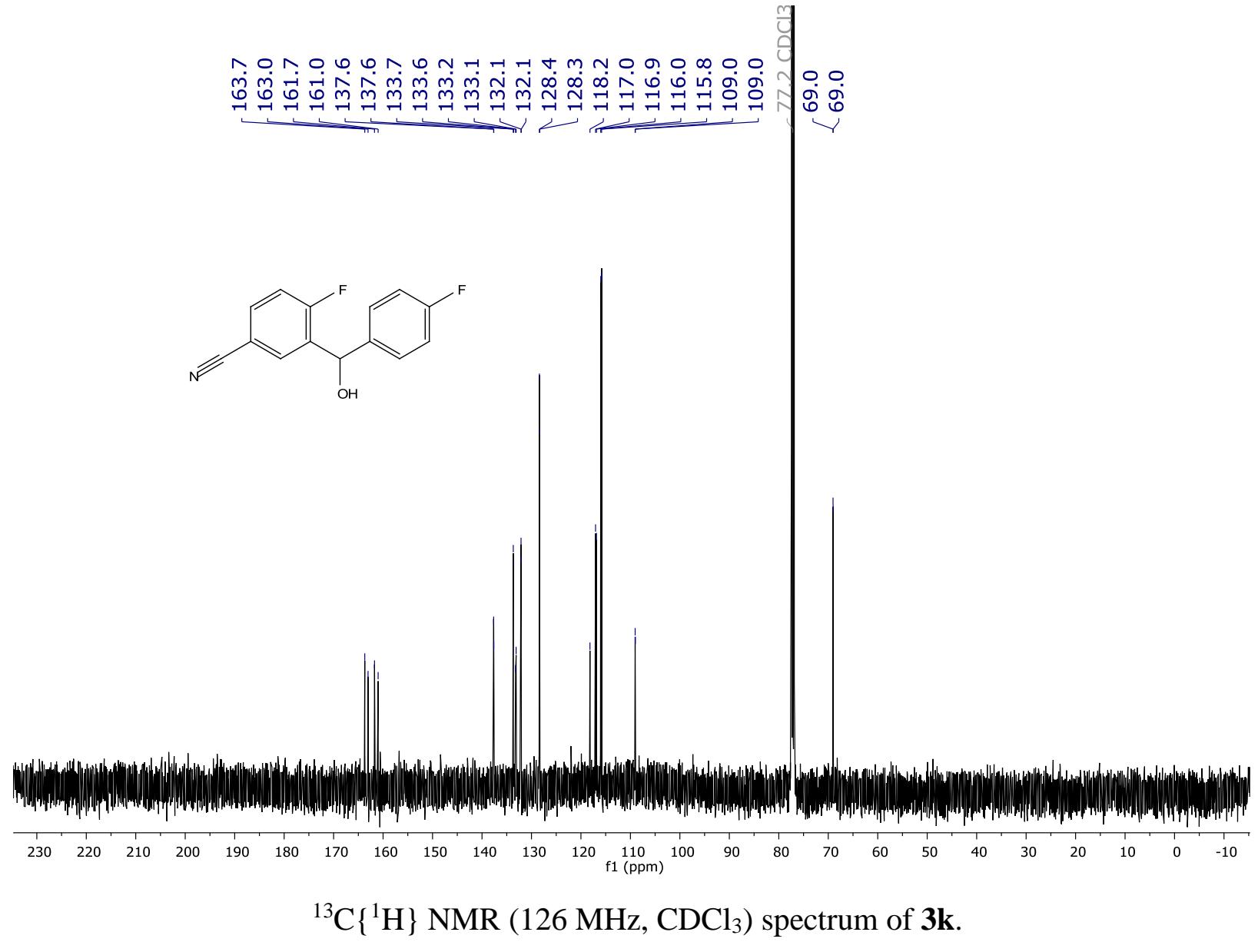




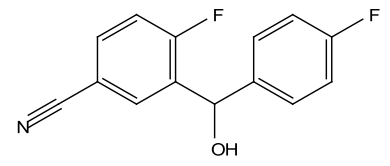

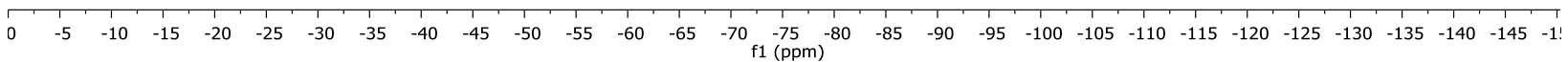

${ }^{19} \mathrm{~F}$ NMR (282 $\left.\mathrm{MHz}, \mathrm{CDCl}_{3}\right)$ spectrum of $\mathbf{3 k}$.

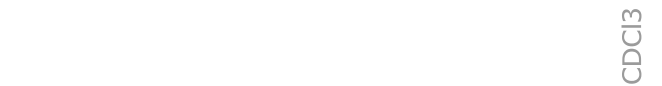

䒛

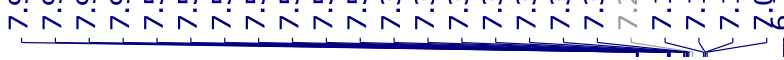

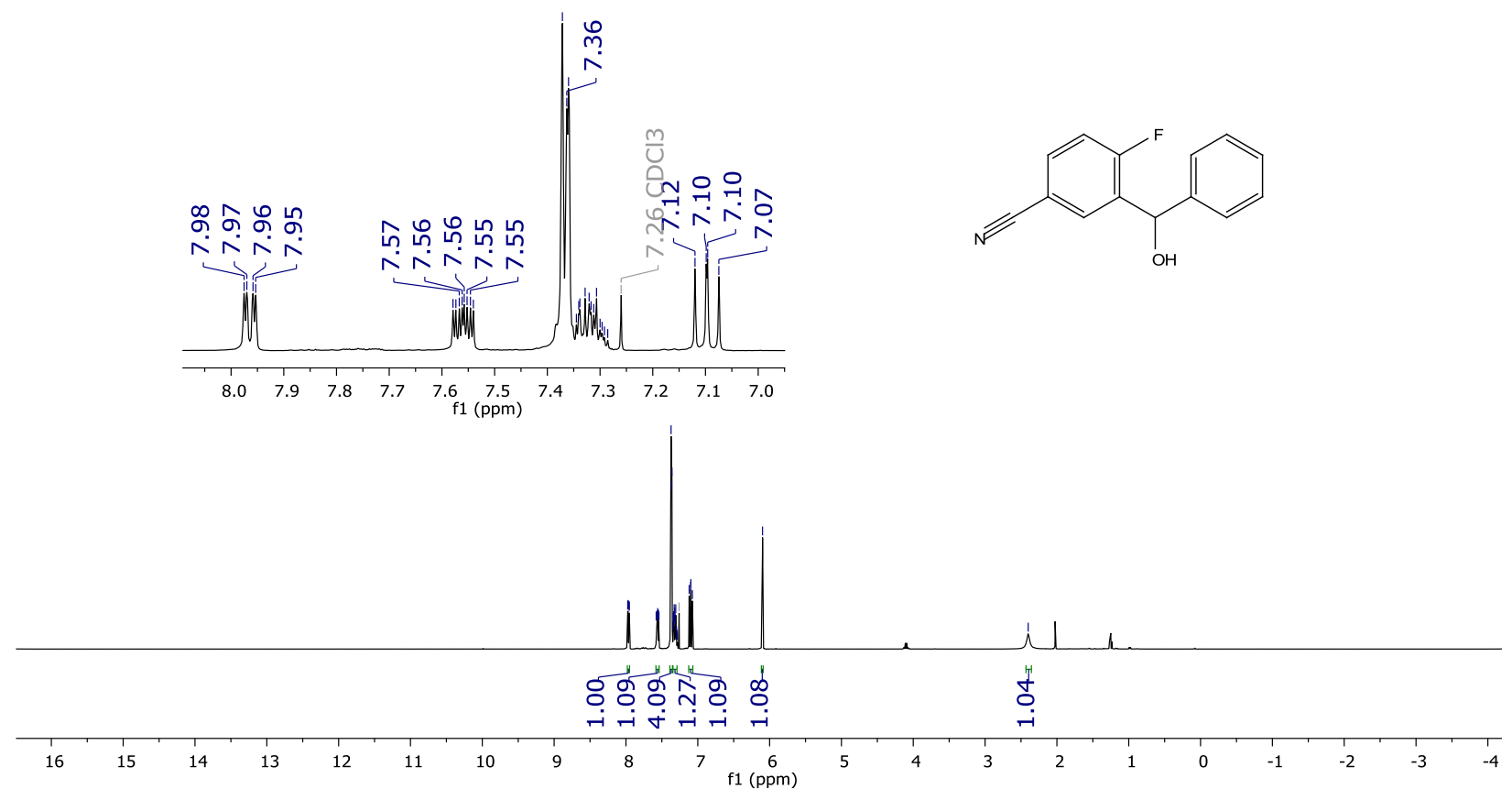

${ }^{1} \mathrm{H} \mathrm{NMR}\left(400 \mathrm{MHz}, \mathrm{CDCl}_{3}\right)$ spectrum of $\mathbf{3 1}$. 


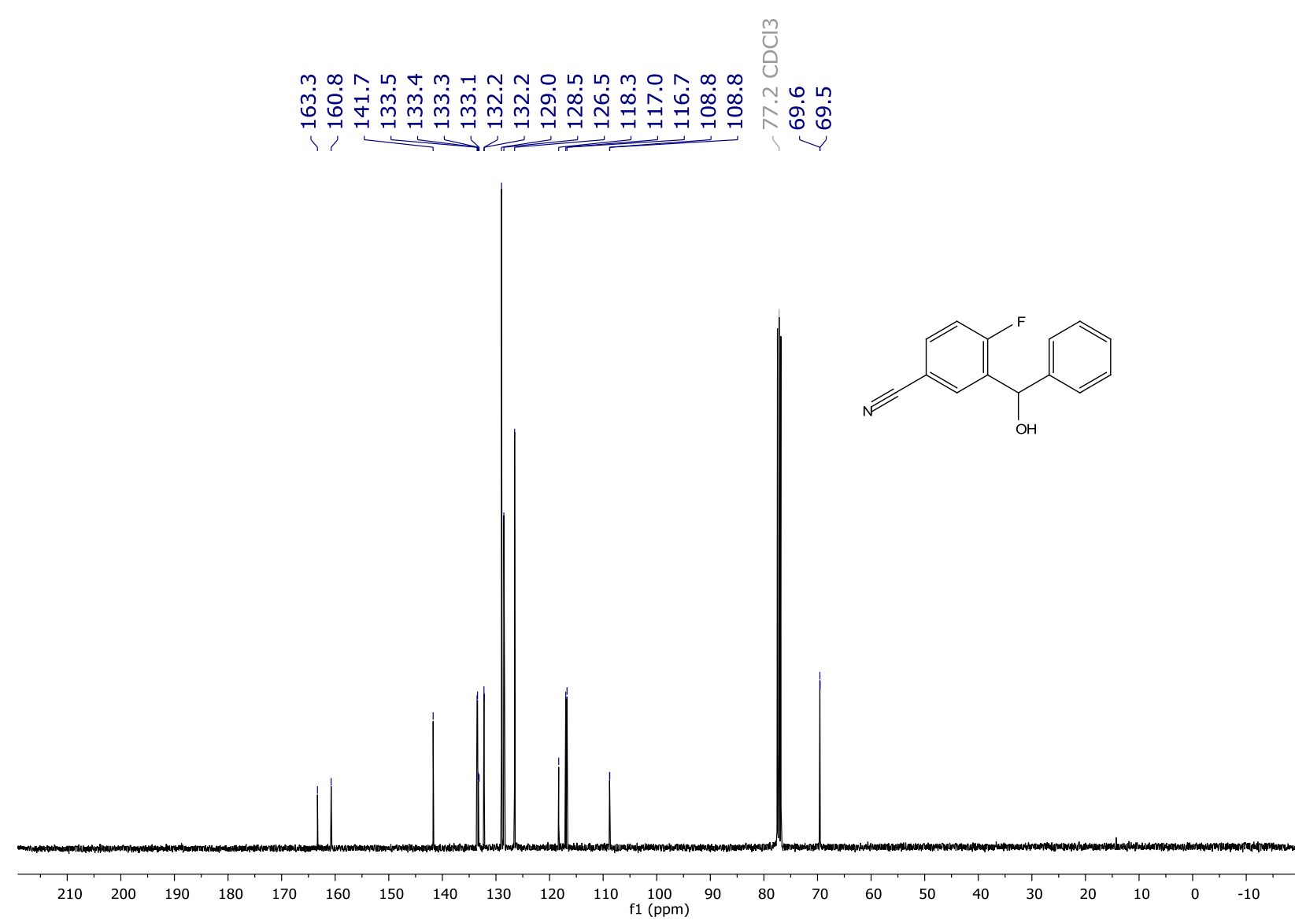

${ }^{13} \mathrm{C}\left\{{ }^{1} \mathrm{H}\right\} \mathrm{NMR}\left(101 \mathrm{MHz}, \mathrm{CDCl}_{3}\right)$ spectrum of $\mathbf{3 1}$.

응좃ํㅅ

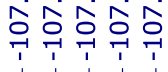
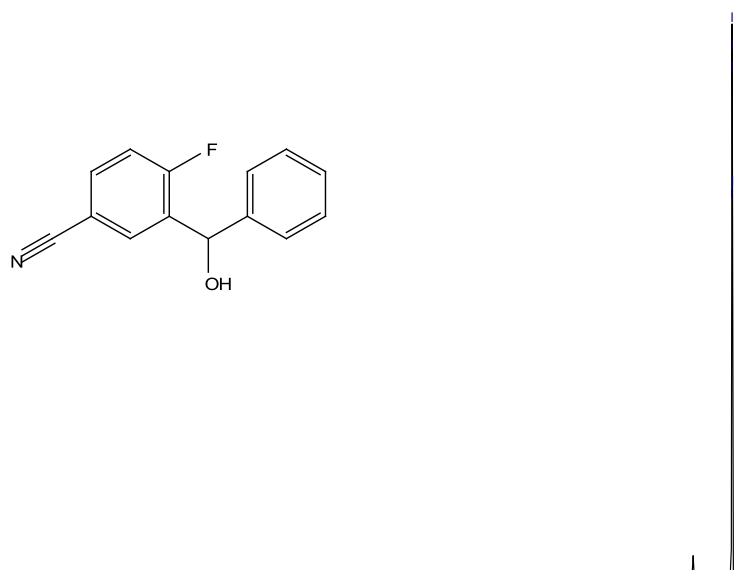

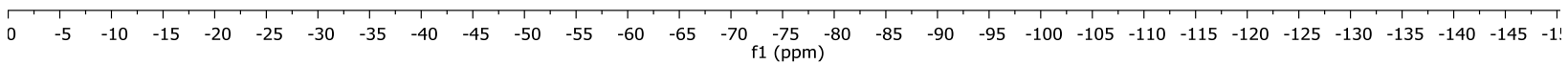

${ }^{19} \mathrm{~F}$ NMR (282 MHz, $\left.\mathrm{CDCl}_{3}\right)$ spectrum of 31. 


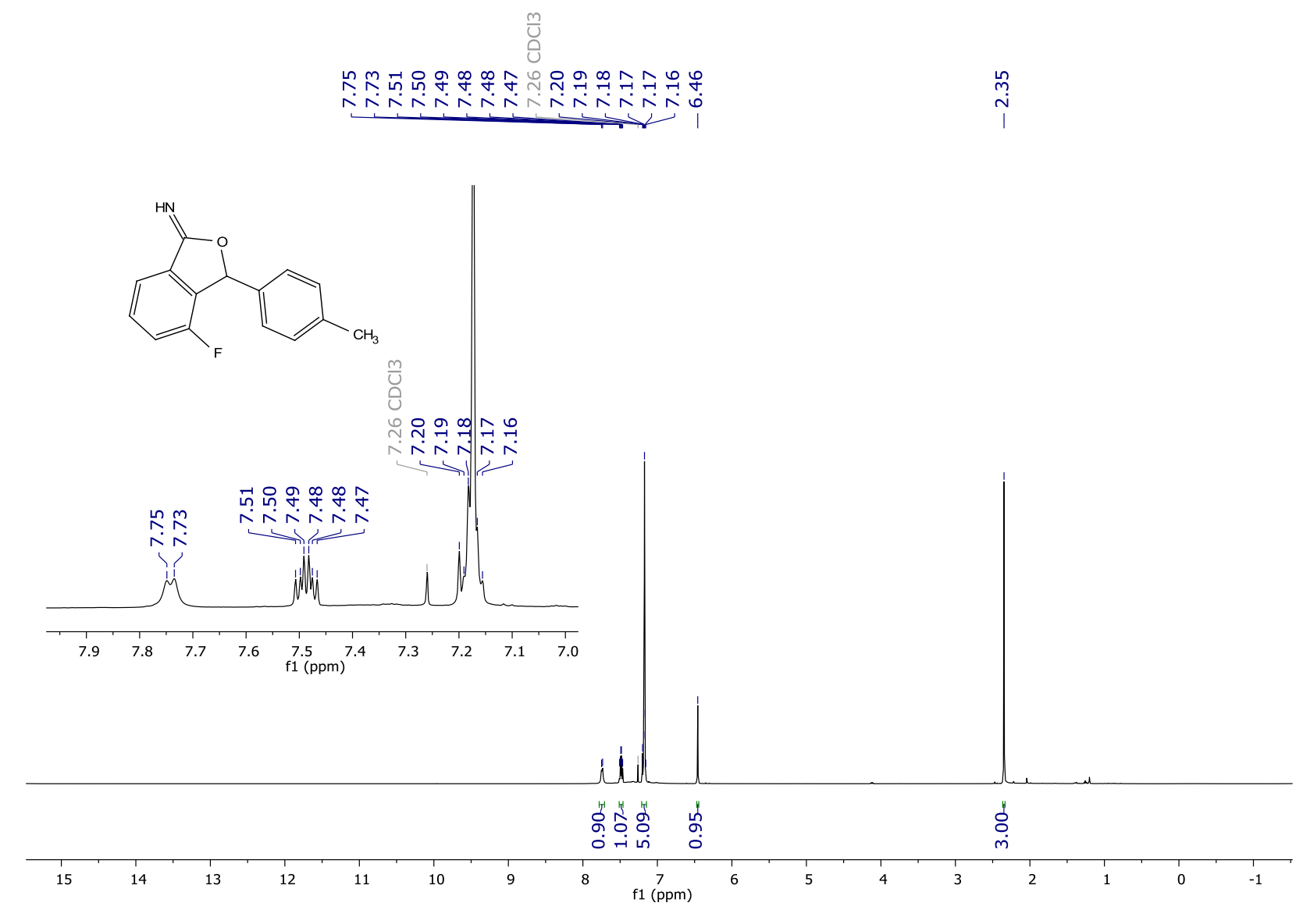

${ }^{1} \mathrm{H}$ NMR (500 MHz, $\left.\mathrm{CDCl}_{3}\right)$ spectrum of $\mathbf{3 m}$.

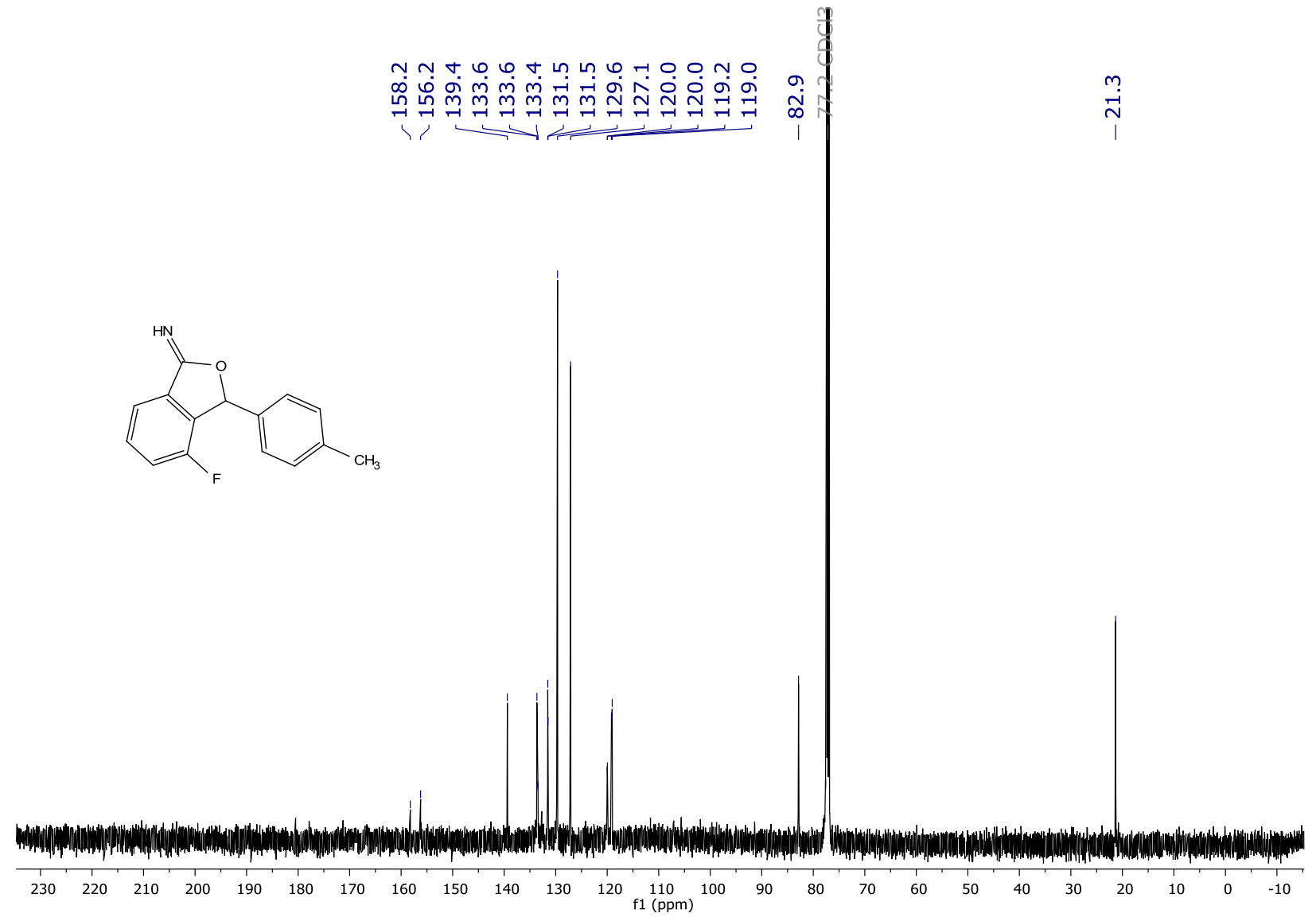

${ }^{13} \mathrm{C}\left\{{ }^{1} \mathrm{H}\right\}$ NMR $\left(126 \mathrm{MHz}, \mathrm{CDCl}_{3}\right)$ spectrum of $\mathbf{3 m}$. 


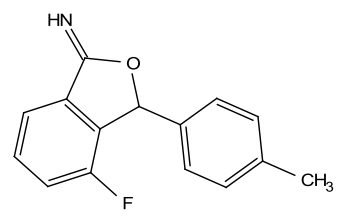

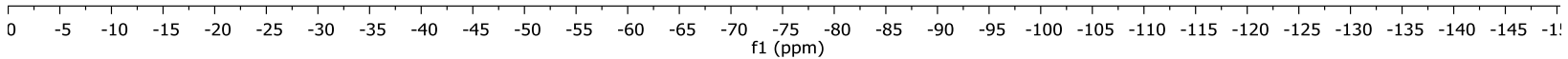

${ }^{19} \mathrm{~F}$ NMR $\left(282 \mathrm{MHz}, \mathrm{CDCl}_{3}\right)$ spectrum of $\mathbf{3 m}$.



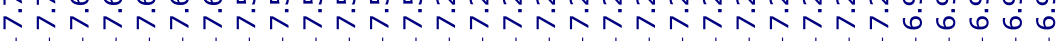
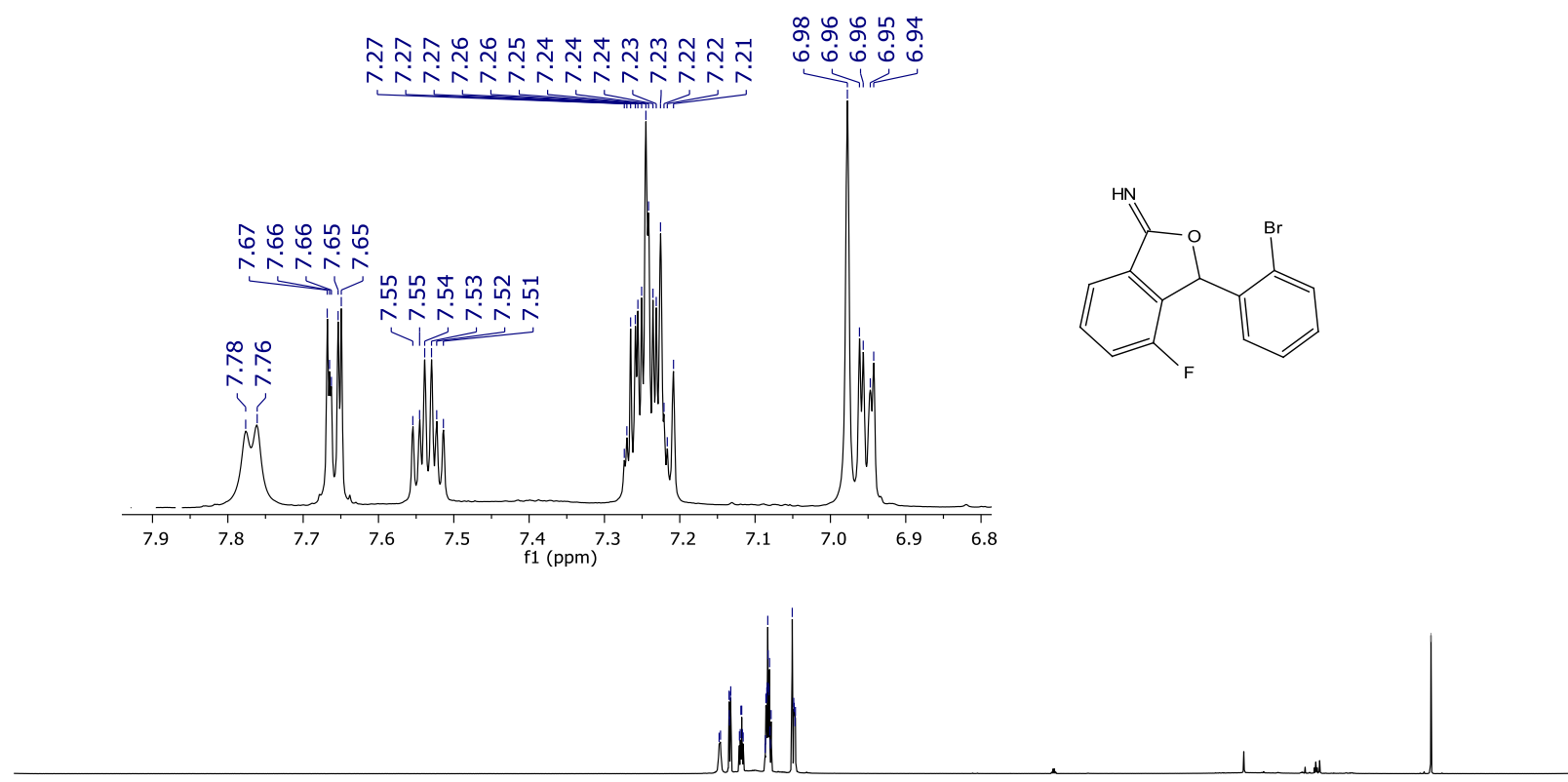

0
0
0

$\begin{array}{llllllllllllllllll}1 & 1 & 14 & 13 & 12 & 11 & 10 & 9 & 8 & 7 & 6 & 5 & 4 & 3 & 2 & 1\end{array}$

${ }^{1} \mathrm{H} \mathrm{NMR}\left(500 \mathrm{MHz}, \mathrm{CDCl}_{3}\right)$ spectrum of $\mathbf{3 n}$. 


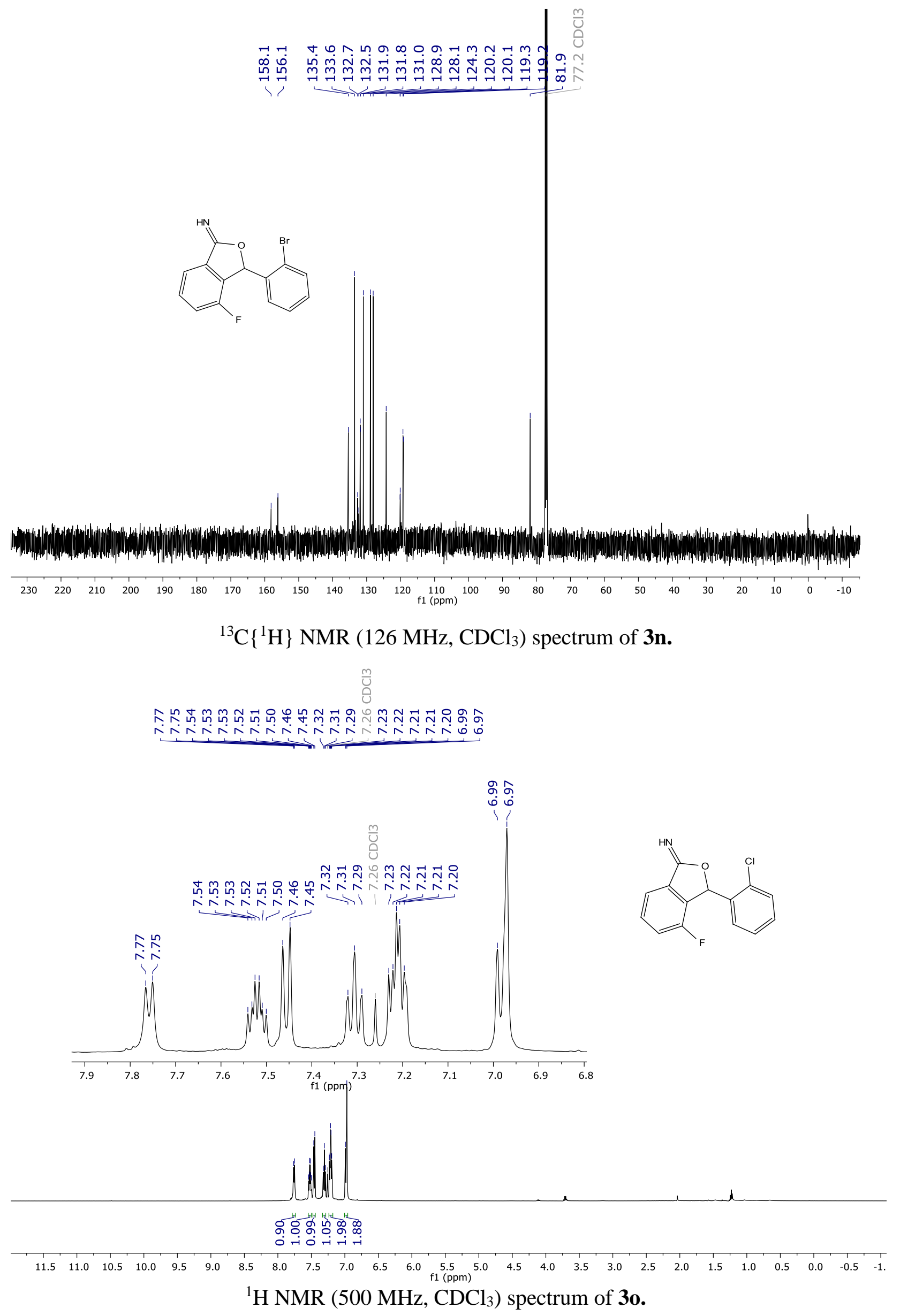



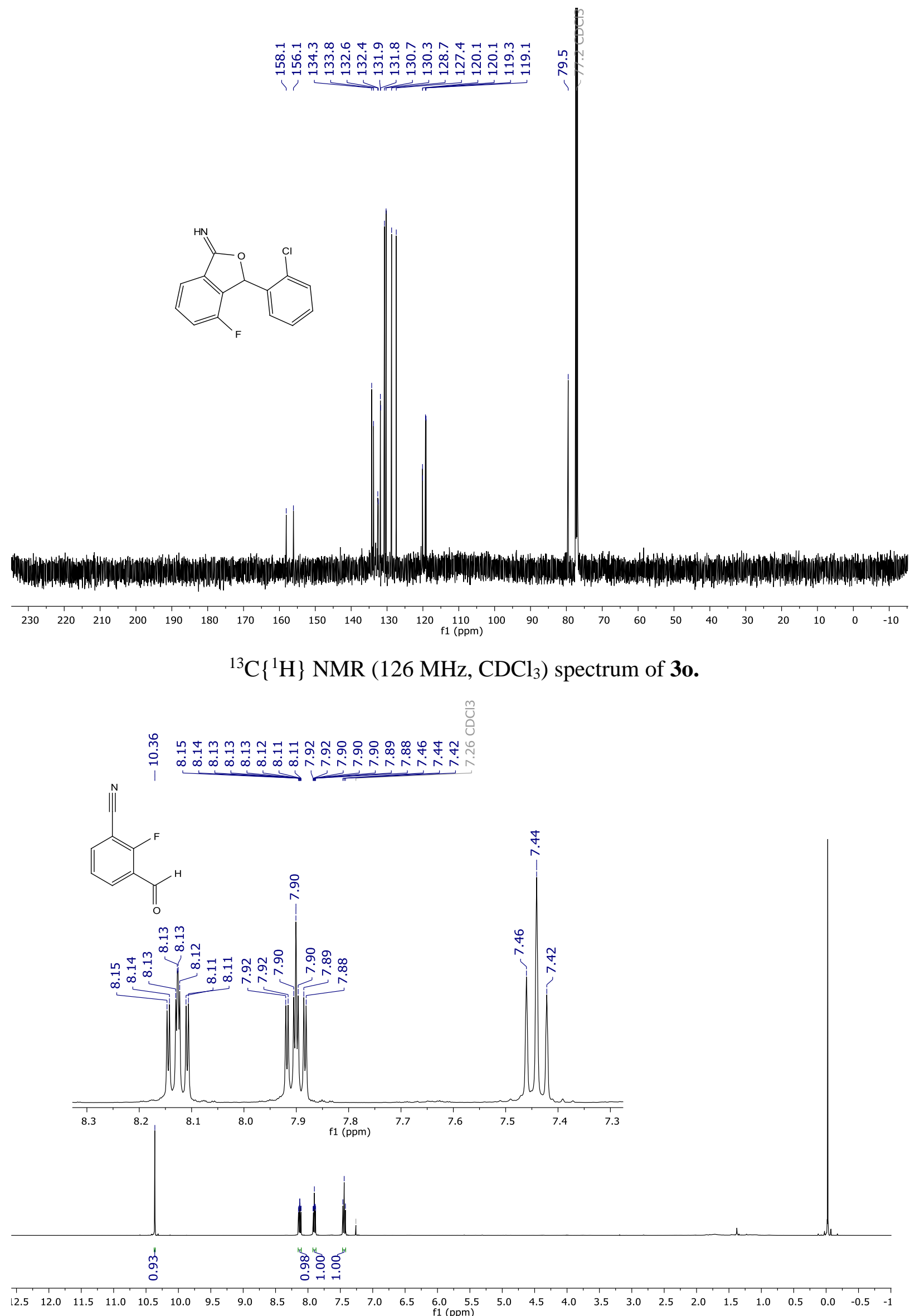

${ }^{1} \mathrm{H}$ NMR (400 MHz, $\mathrm{CDCl}_{3}$ ) spectrum of 3p. 
<smiles>N#Cc1cccc(C=O)c1F</smiles>

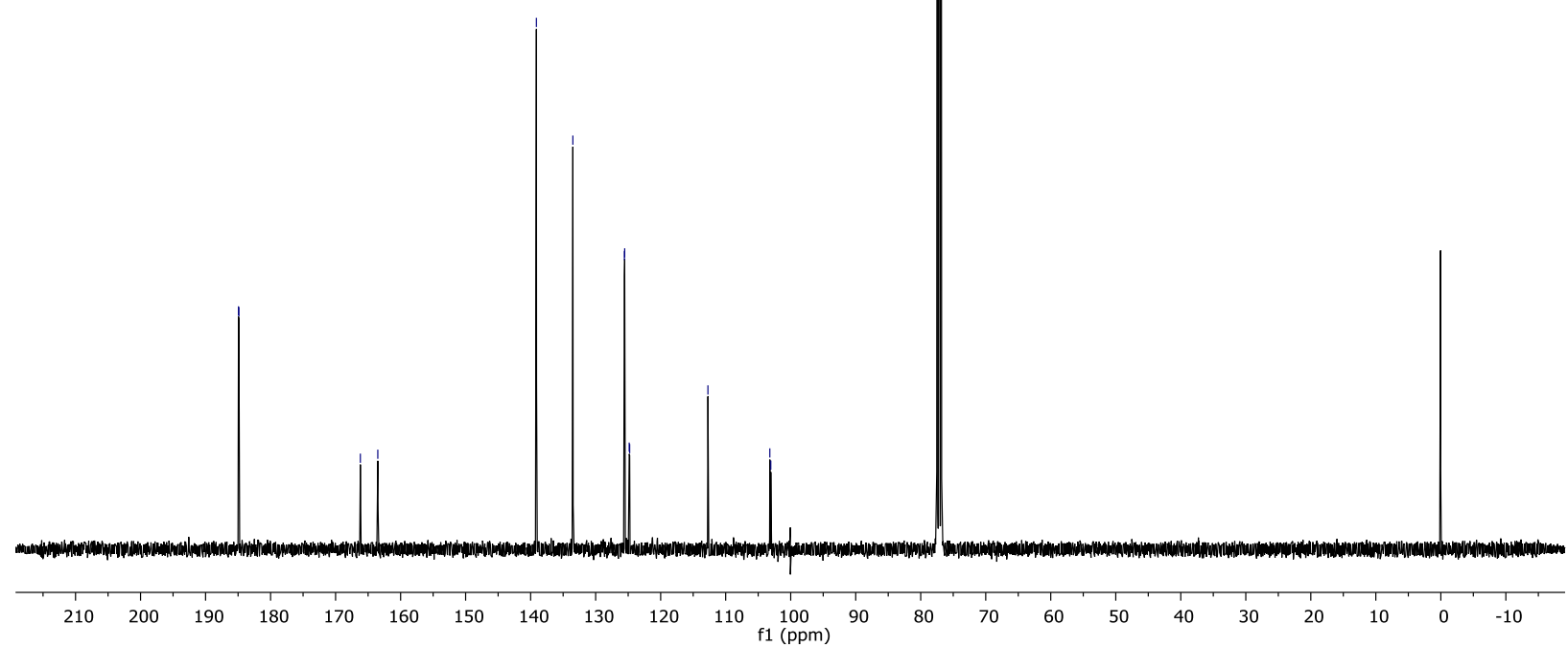

${ }^{13} \mathrm{C}\left\{{ }^{1} \mathrm{H}\right\}$ NMR $\left(101 \mathrm{MHz}, \mathrm{CDCl}_{3}\right)$ spectrum of $\mathbf{3 p}$.

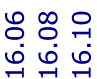

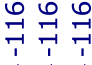

$r$

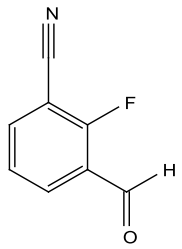

\begin{tabular}{llllllllllllllllllllllllllllllllllllllllllll}
\hline 0 & -5 & -10 & -15 & -20 & -25 & -30 & -35 & -40 & -45 & -50 & -55 & -60 & -65 & -70 & -75 & -80 & -85 & -90 & -95 & -100 & -105 & -110 & -115 & -120 & -125 & -130 & -135 & -140 & -145 & -1
\end{tabular}

${ }^{19} \mathrm{~F}$ NMR (282 $\left.\mathrm{MHz}, \mathrm{CDCl}_{3}\right)$ spectrum of $\mathbf{3 p}$. 


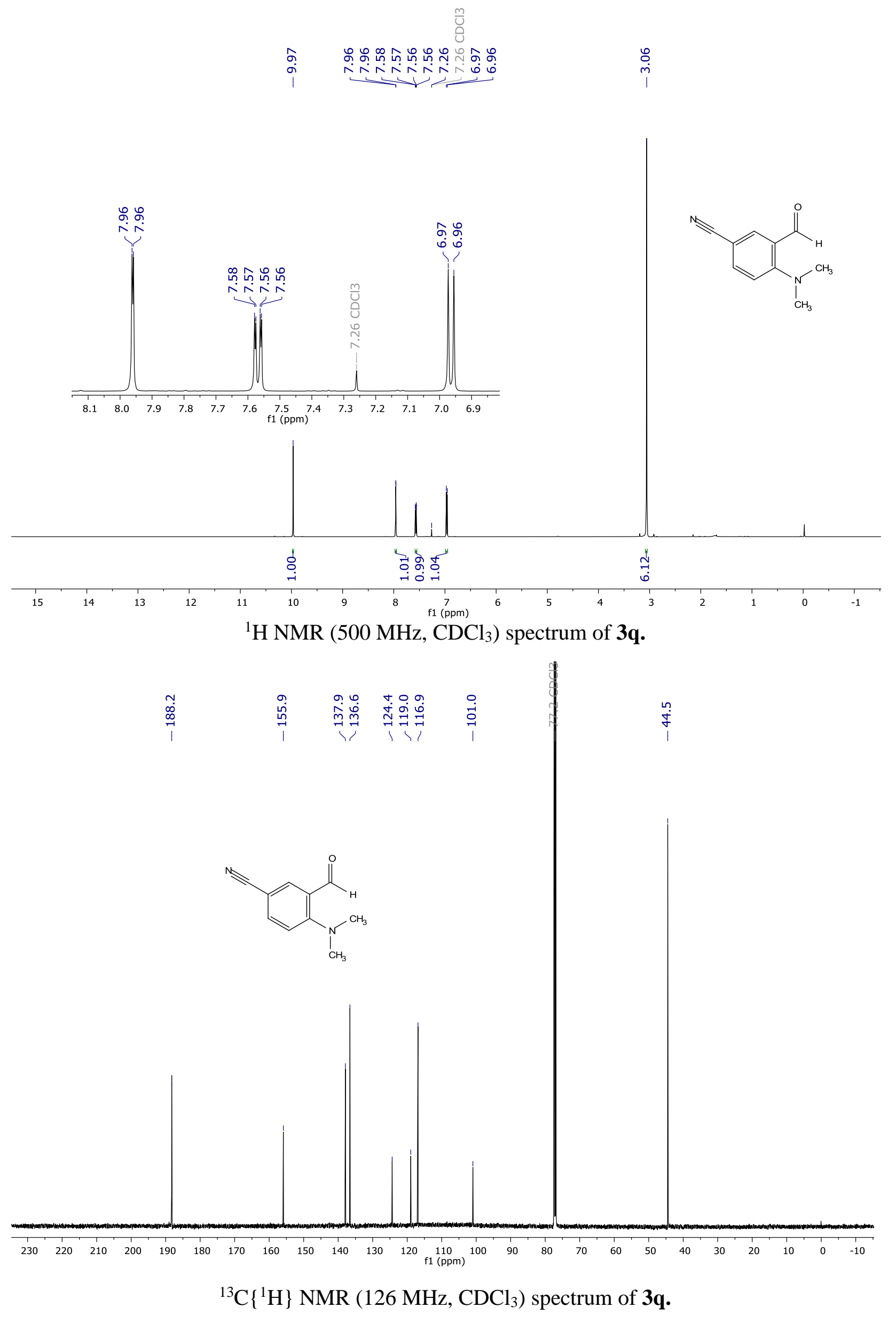




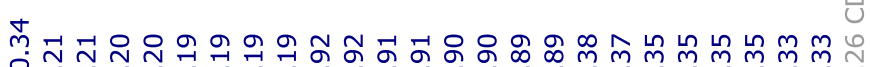

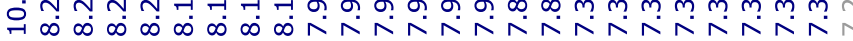

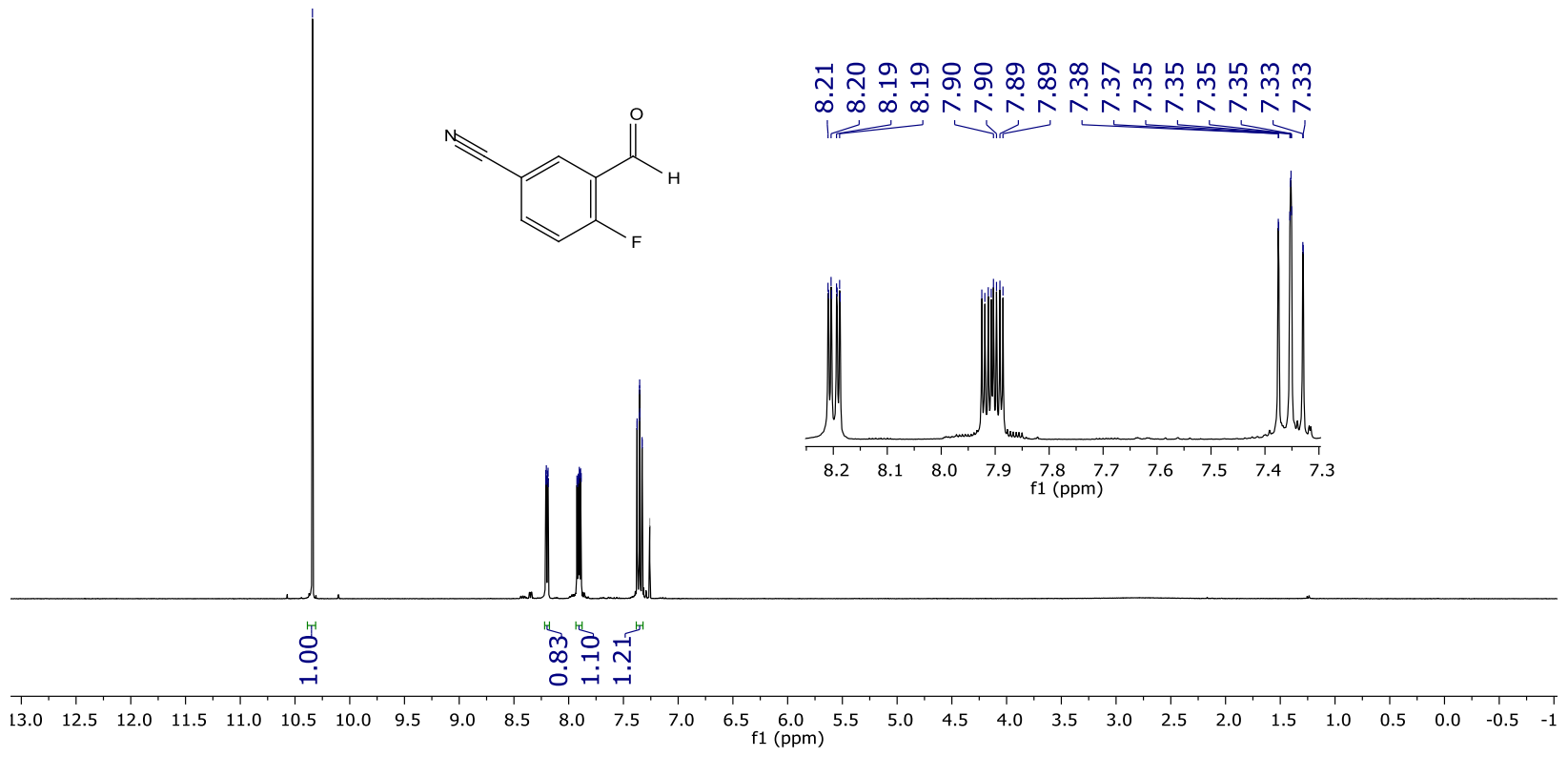

${ }^{1} \mathrm{H}$ NMR (400 MHz, $\mathrm{CDCl}_{3}$ ) spectrum of $\mathbf{3 r}$.

이 $\quad$ 구

许<smiles>N#Cc1ccc(F)c(C=O)c1</smiles>

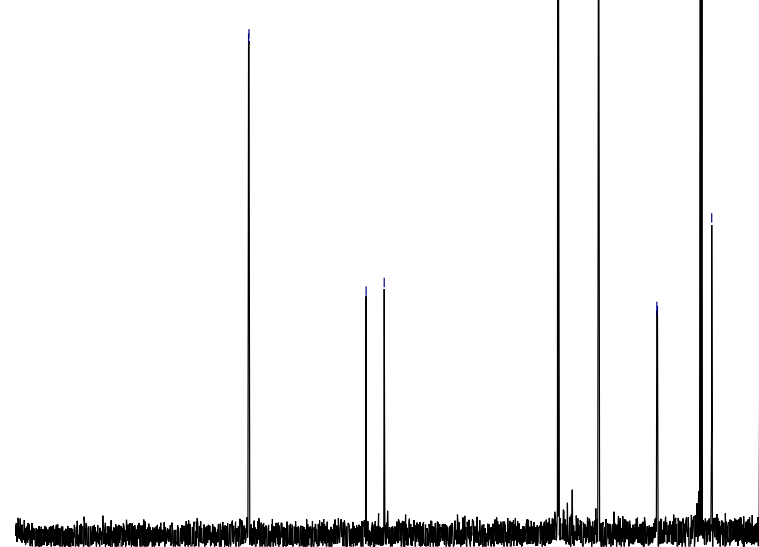

$\begin{array}{llllllllllll}210 & 200 & 190 & 180 & 170 & 160 & 150 & 140 & 130 & 120 & 110 & 100\end{array}$

${ }^{13} \mathrm{C}\left\{{ }^{1} \mathrm{H}\right\}$ NMR $\left(101 \mathrm{MHz}, \mathrm{CDCl}_{3}\right)$ spectrum of $\mathbf{3 r}$. 

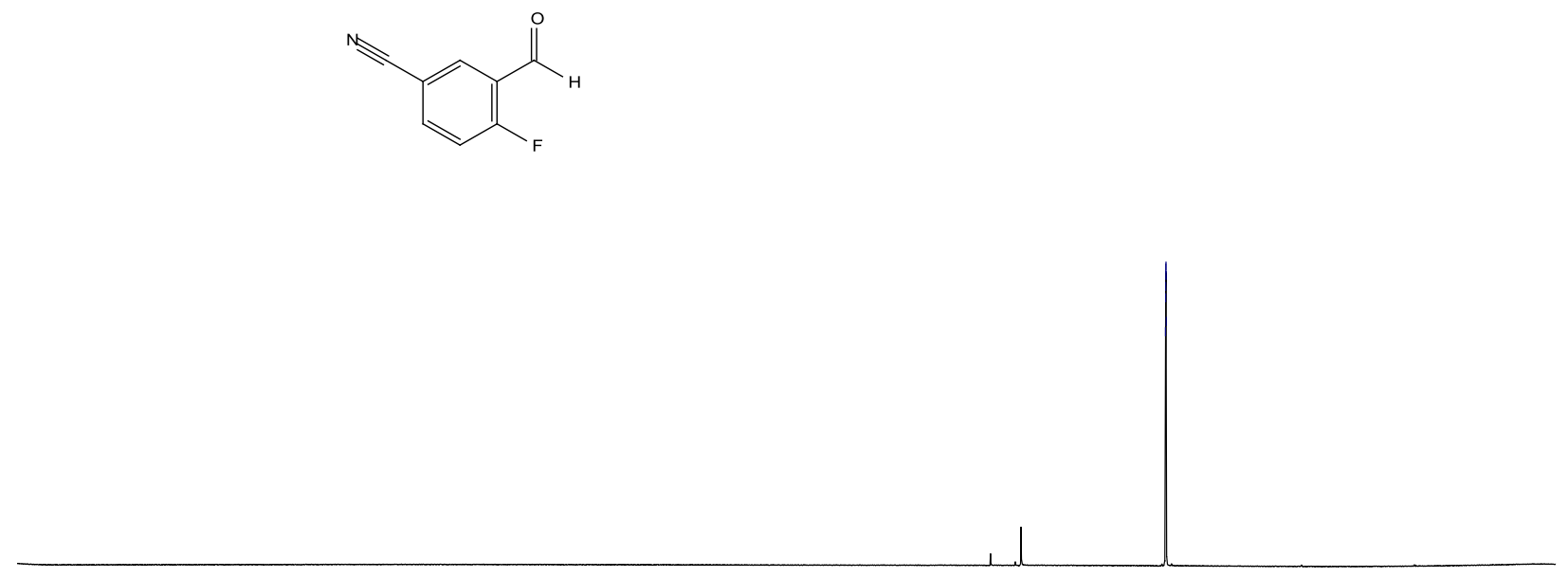

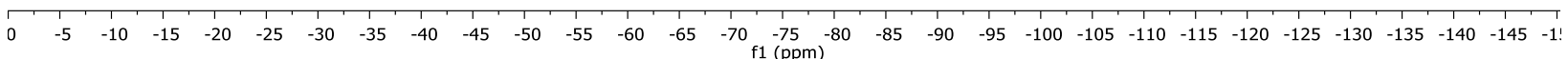

${ }^{19} \mathrm{~F} \mathrm{NMR}\left(282 \mathrm{MHz}, \mathrm{CDCl}_{3}\right)$ spectrum of $\mathbf{3 r}$.

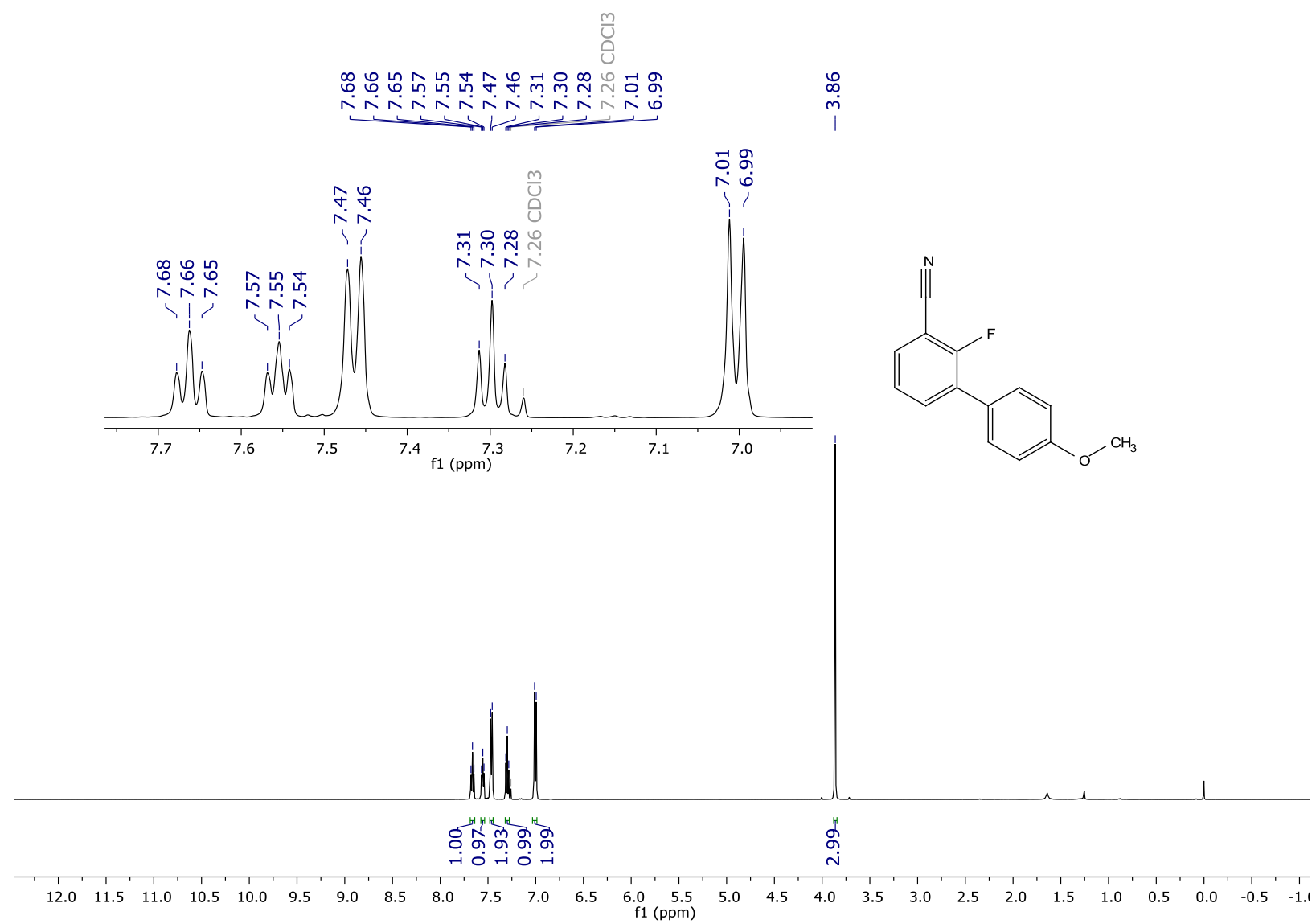

${ }^{1} \mathrm{H}$ NMR $\left(500 \mathrm{MHz}, \mathrm{CDCl}_{3}\right)$ spectrum of $\mathbf{3 s}$. 


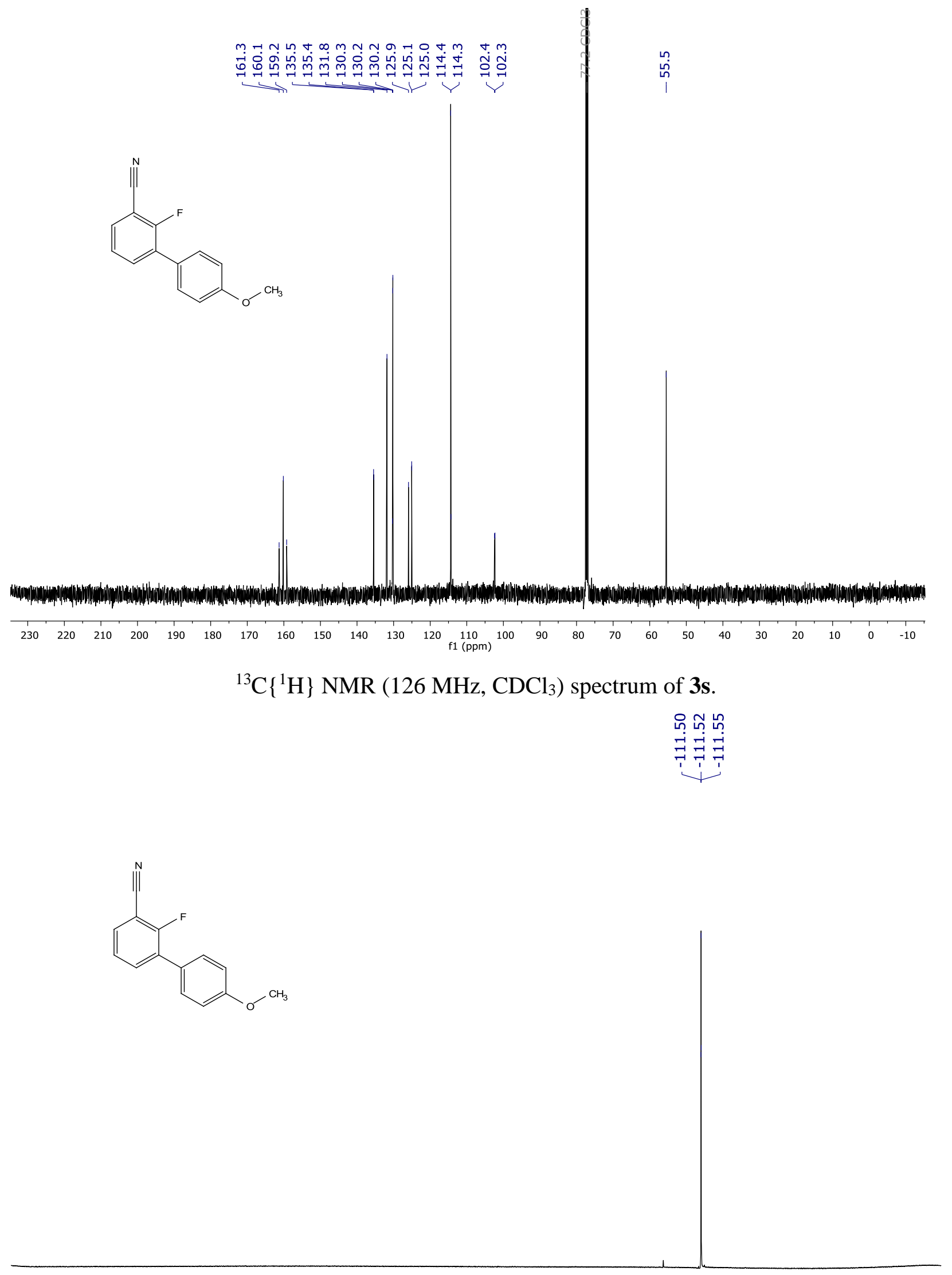

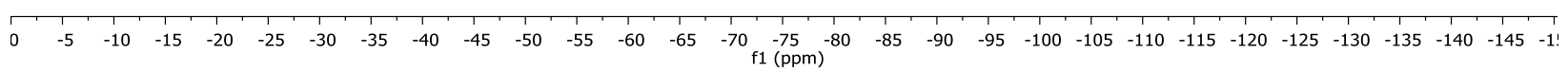

${ }^{19} \mathrm{~F}$ NMR (282 MHz, $\left.\mathrm{CDCl}_{3}\right)$ spectrum of $3 \mathbf{s}$. 


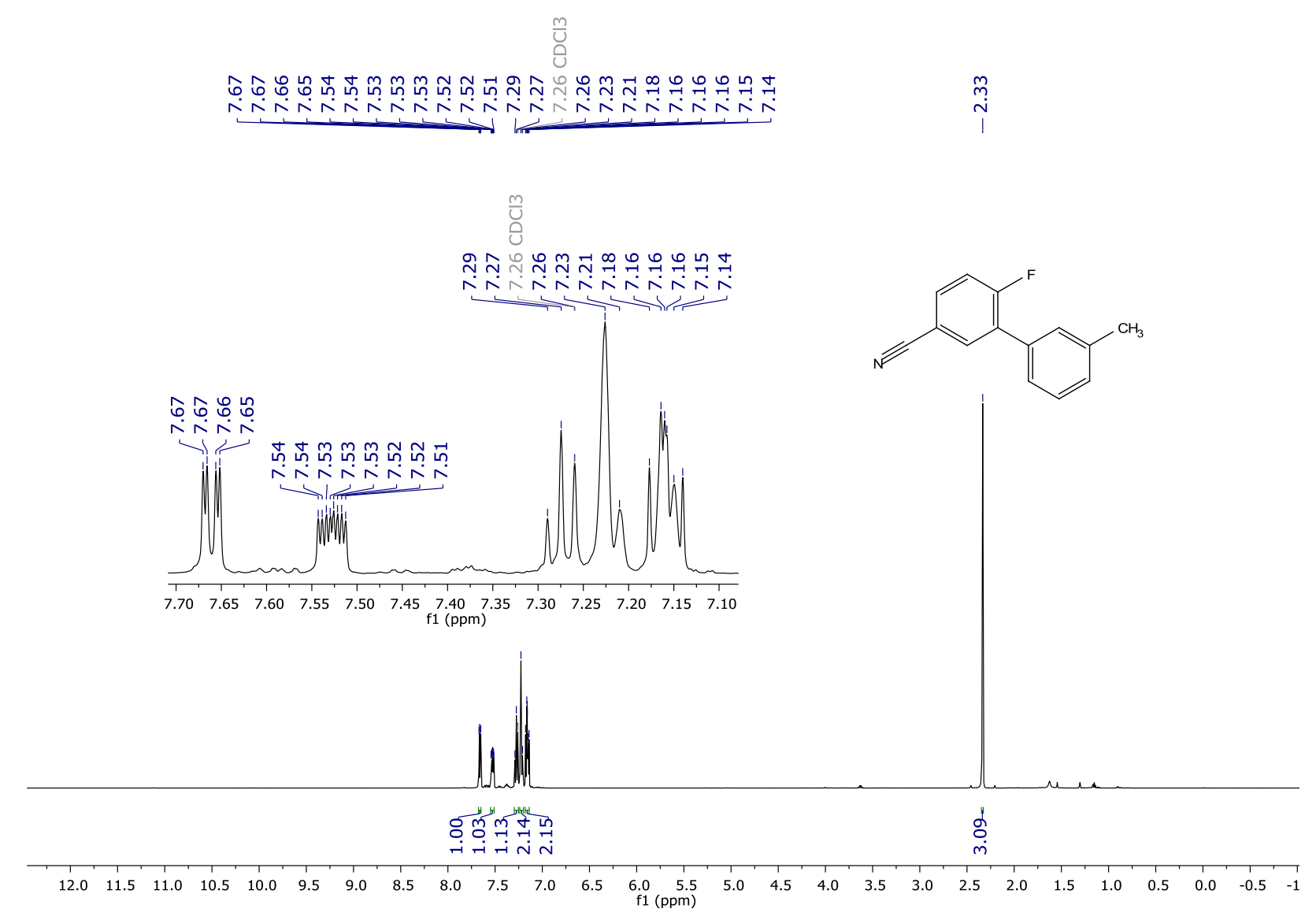

${ }^{1} \mathrm{H}$ NMR $\left(500 \mathrm{MHz}, \mathrm{CDCl}_{3}\right)$ spectrum of $3 \mathbf{3 t}$

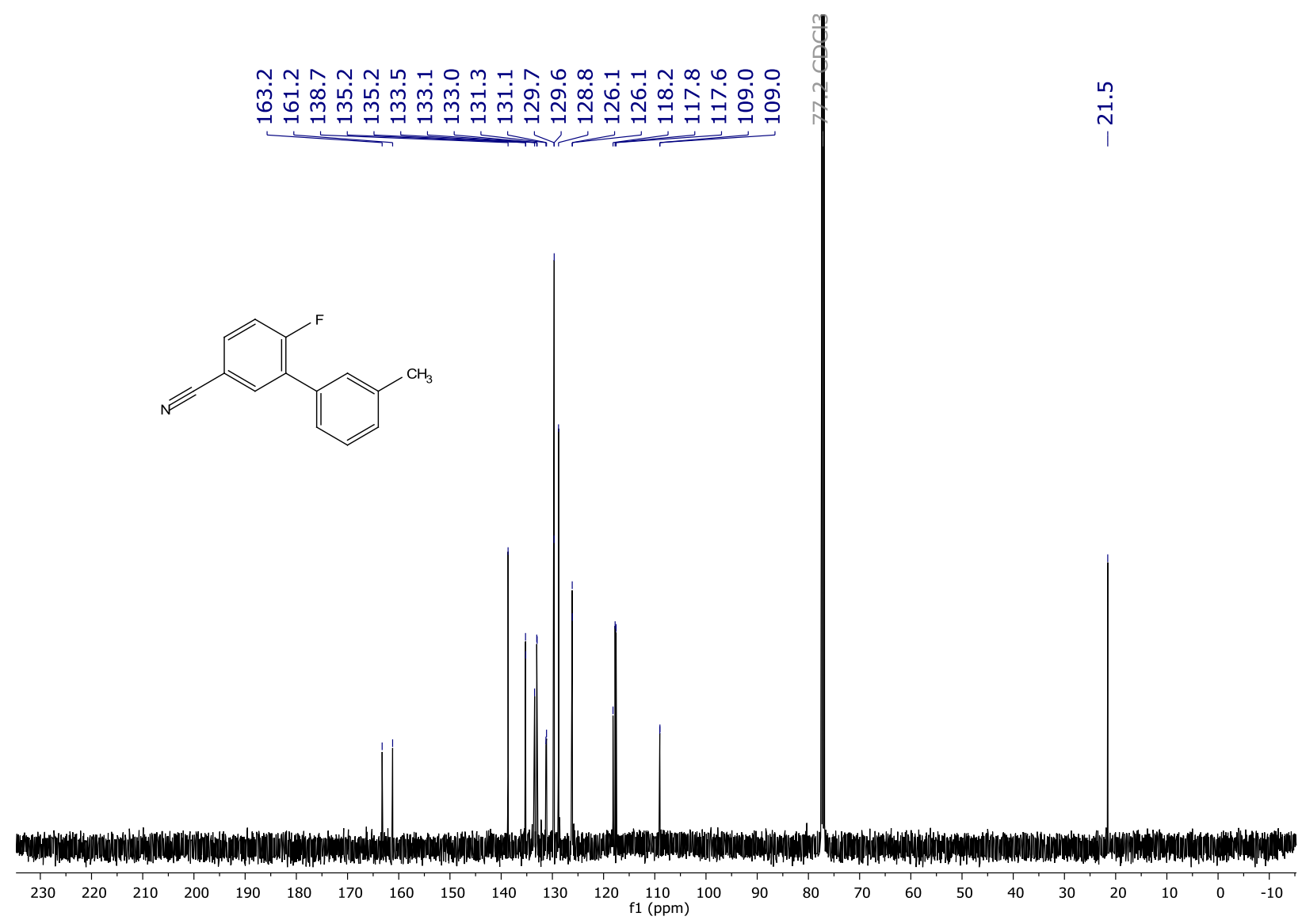

${ }^{13} \mathrm{C}\left\{{ }^{1} \mathrm{H}\right\}$ NMR (126 MHz, $\left.\mathrm{CDCl}_{3}\right)$ spectrum of $\mathbf{3 t}$. 

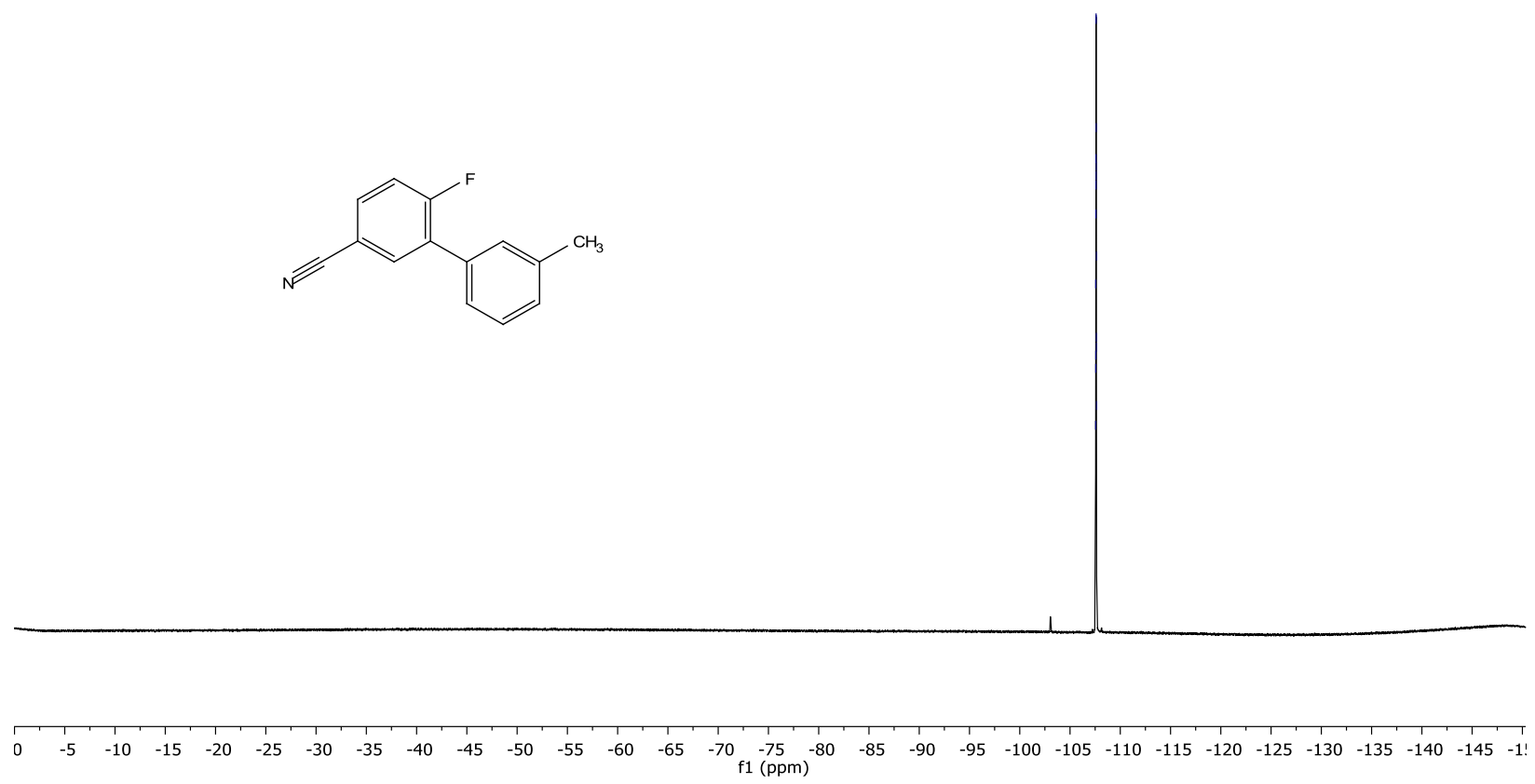

${ }^{19} \mathrm{~F}$ NMR (282 MHz, $\mathrm{CDCl}_{3}$ ) spectrum of $3 t$.

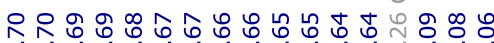

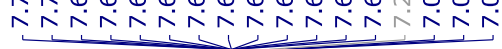
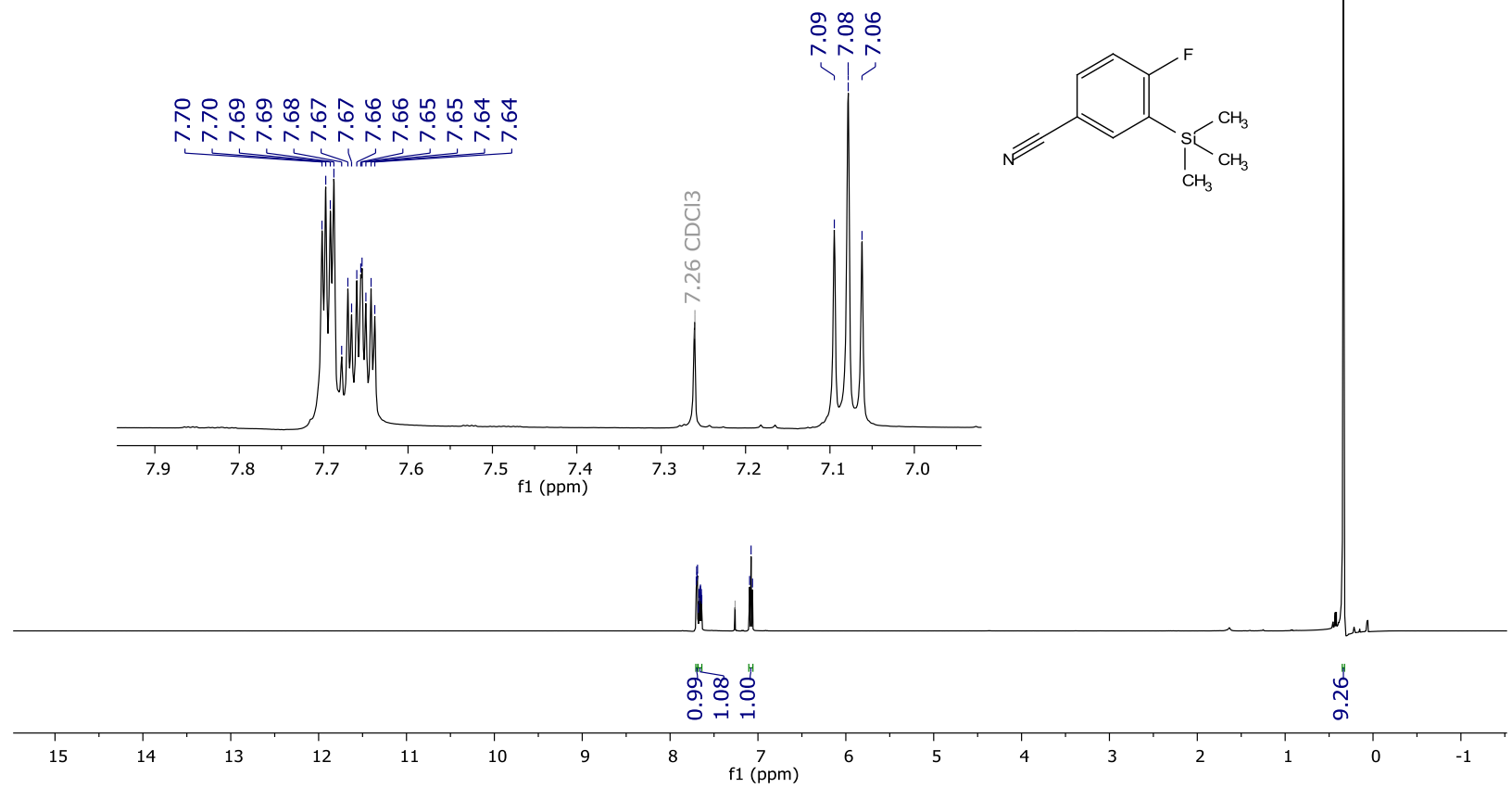

${ }^{1} \mathrm{H}$ NMR $\left(500 \mathrm{MHz}, \mathrm{CDCl}_{3}\right)$ spectrum of $\mathbf{3 u}$. 


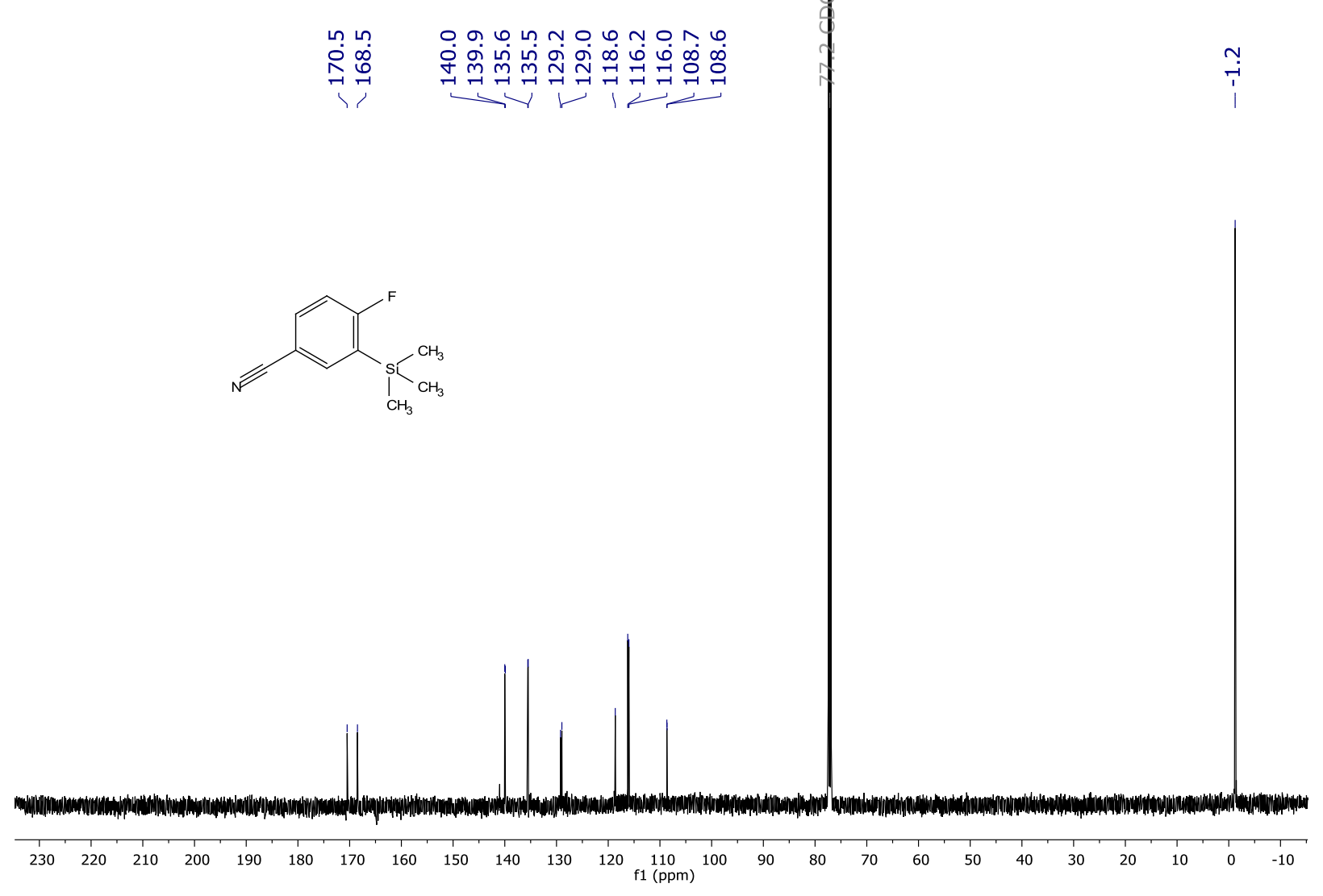

${ }^{13} \mathrm{C}\left\{{ }^{1} \mathrm{H}\right\}$ NMR $\left(126 \mathrm{MHz}, \mathrm{CDCl}_{3}\right)$ spectrum of $\mathbf{3 u}$.

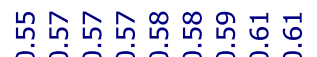

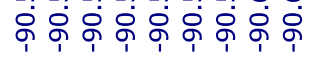

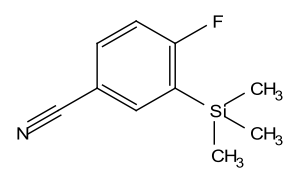

\begin{tabular}{|lllllllllllllllllllllllllllllllllllllllllllllllllllllllll}
\hline 0 & -5 & -10 & -15 & -20 & -25 & -30 & -35 & -40 & -45 & -50 & -55 & -60 & -65 & -70 & -75 & -80 & -85 & -90 & -95 & -100 & -105 & -110 & -115 & -120 & -125 & -130 & -135 & -140 & -145 & -1
\end{tabular}

${ }^{19} \mathrm{~F}$ NMR (282 MHz, $\mathrm{CDCl}_{3}$ ) spectrum of $\mathbf{3 u}$. 


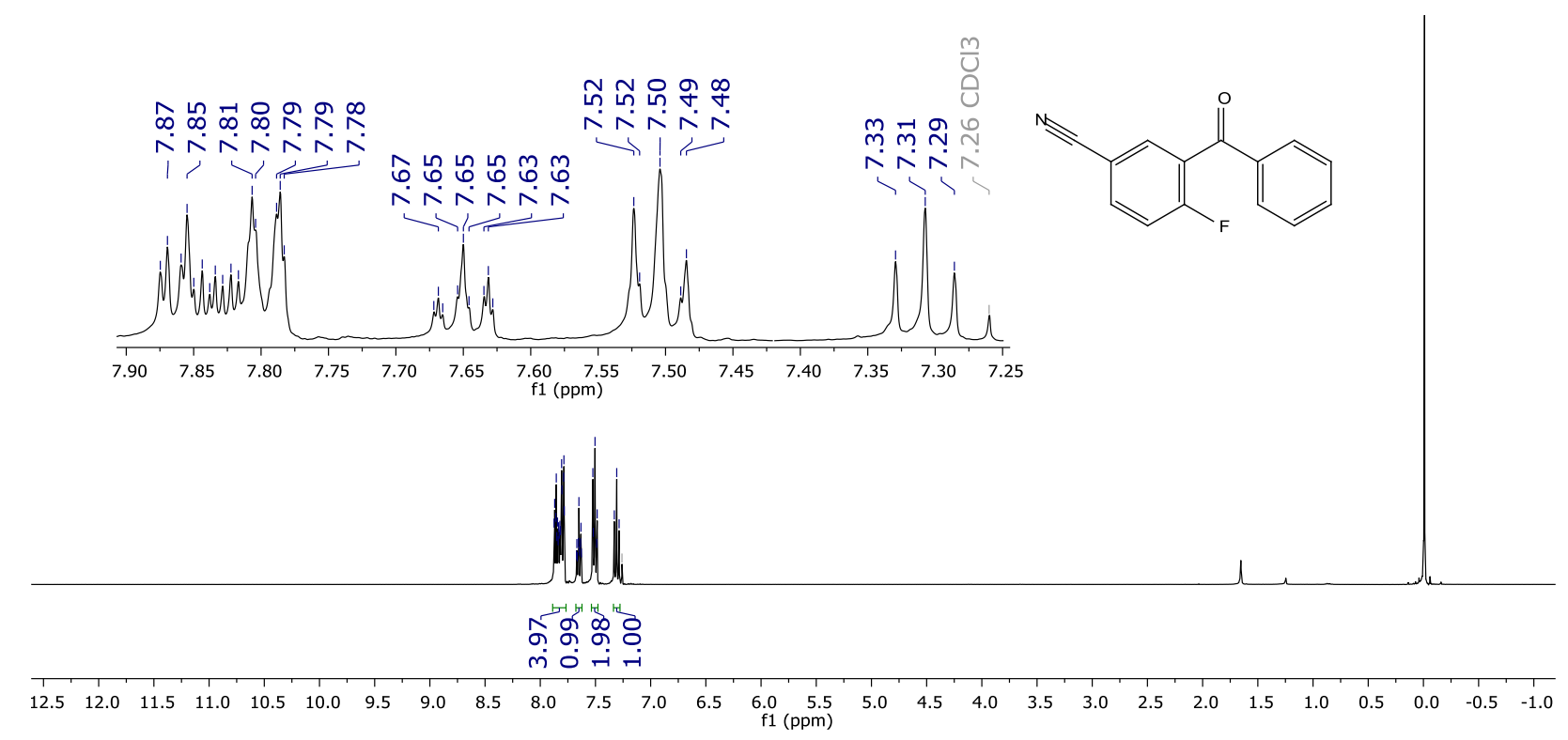

${ }^{1} \mathrm{H}$ NMR $\left(400 \mathrm{MHz}, \mathrm{CDCl}_{3}\right)$ spectrum of $\mathbf{3 v}$.
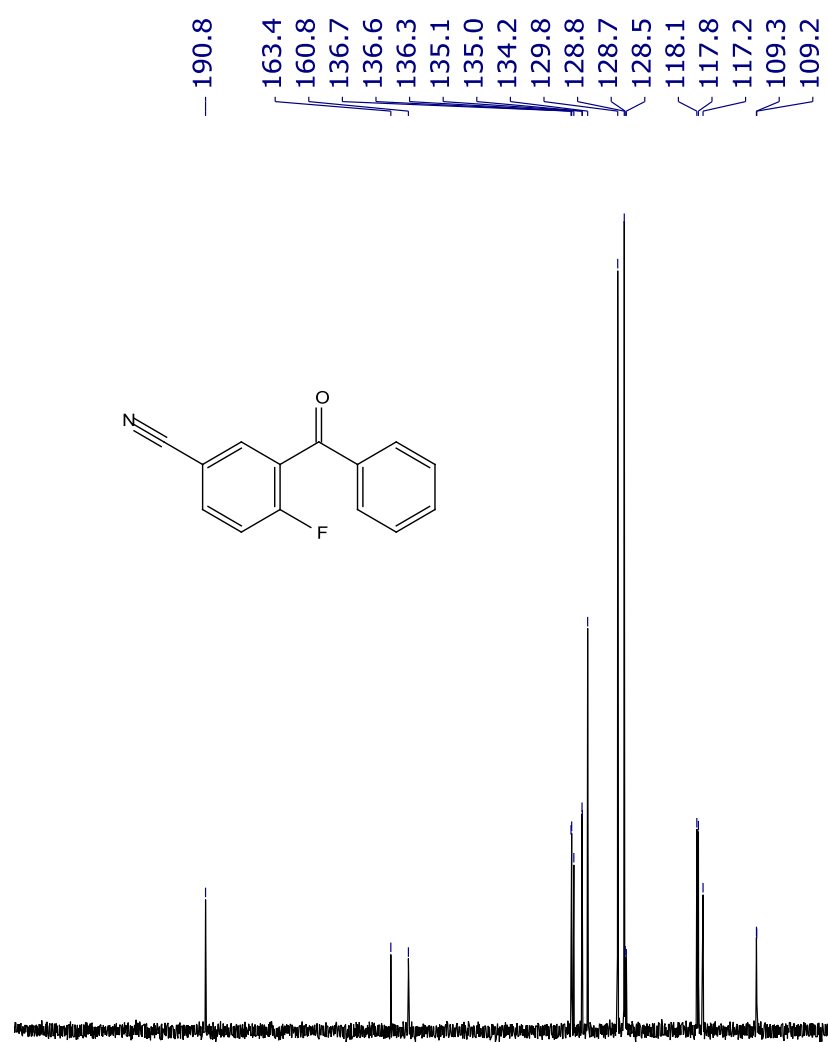

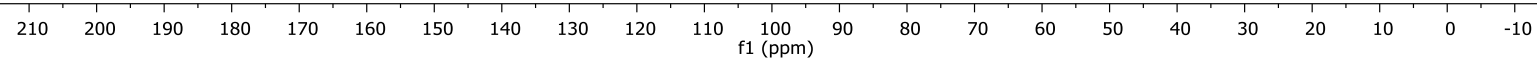

${ }^{13} \mathrm{C}\left\{{ }^{1} \mathrm{H}\right\}$ NMR (101 MHz, $\left.\mathrm{CDCl}_{3}\right)$ spectrum of $\mathbf{3 v}$. 

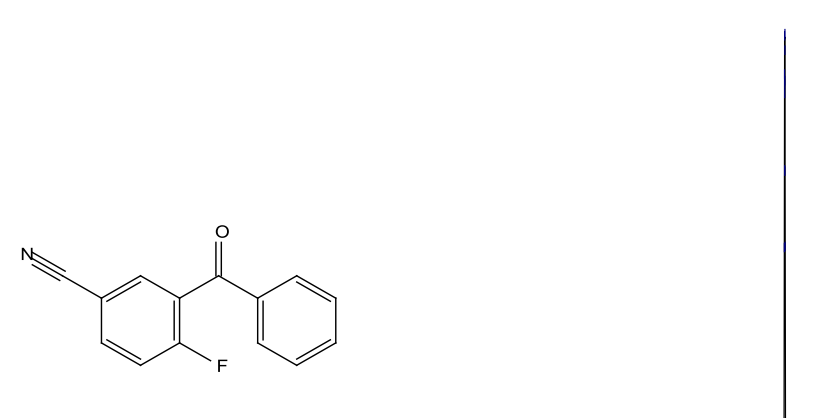

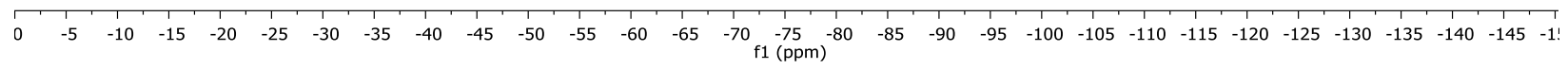

${ }^{19} \mathrm{~F} \mathrm{NMR}\left(282 \mathrm{MHz}, \mathrm{CDCl}_{3}\right)$ spectrum of $\mathbf{3 v}$.

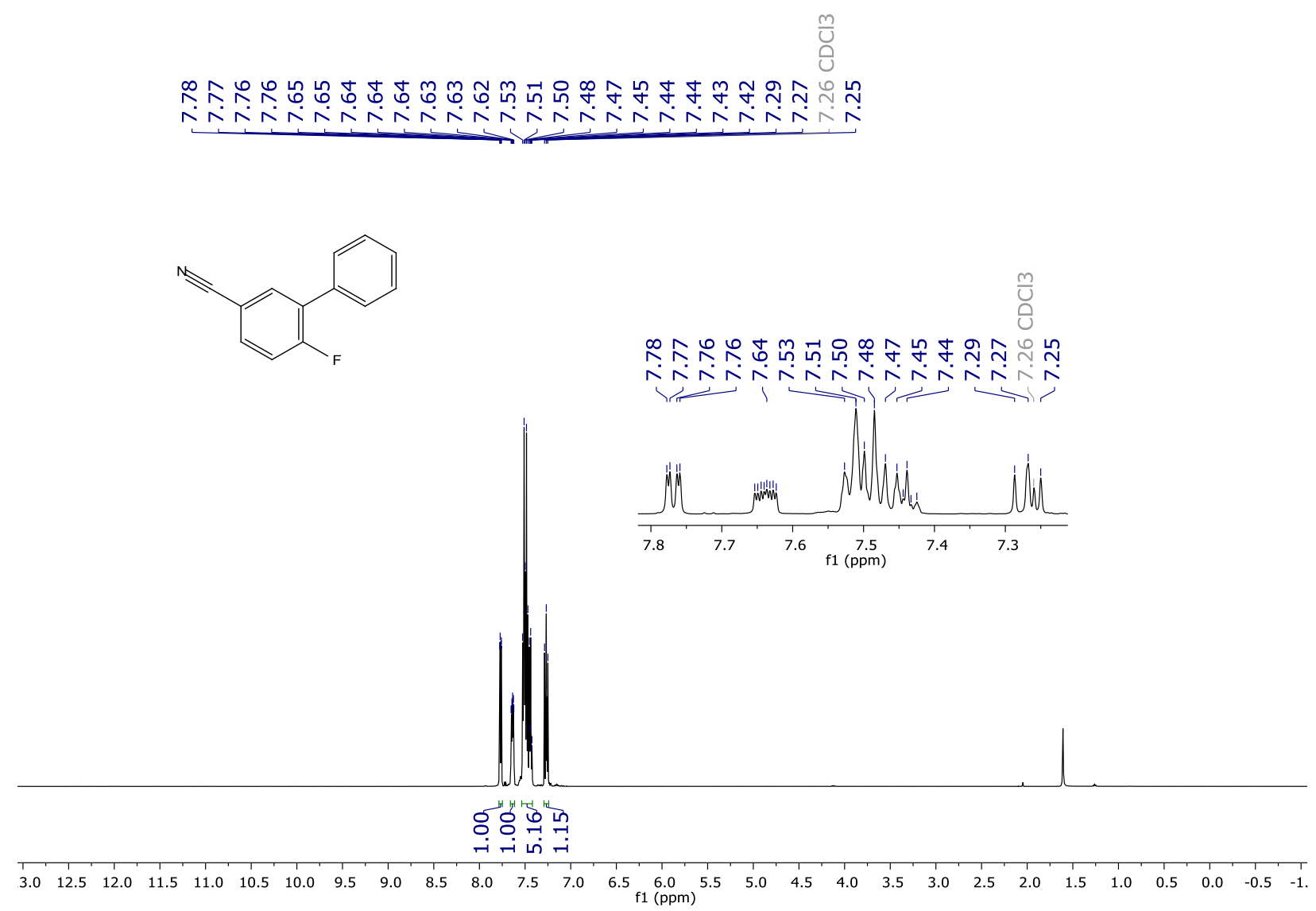

${ }^{1} \mathrm{H} \mathrm{NMR}\left(400 \mathrm{MHz}, \mathrm{CDCl}_{3}\right)$ spectrum of 3w. 


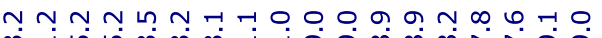

ஸिન
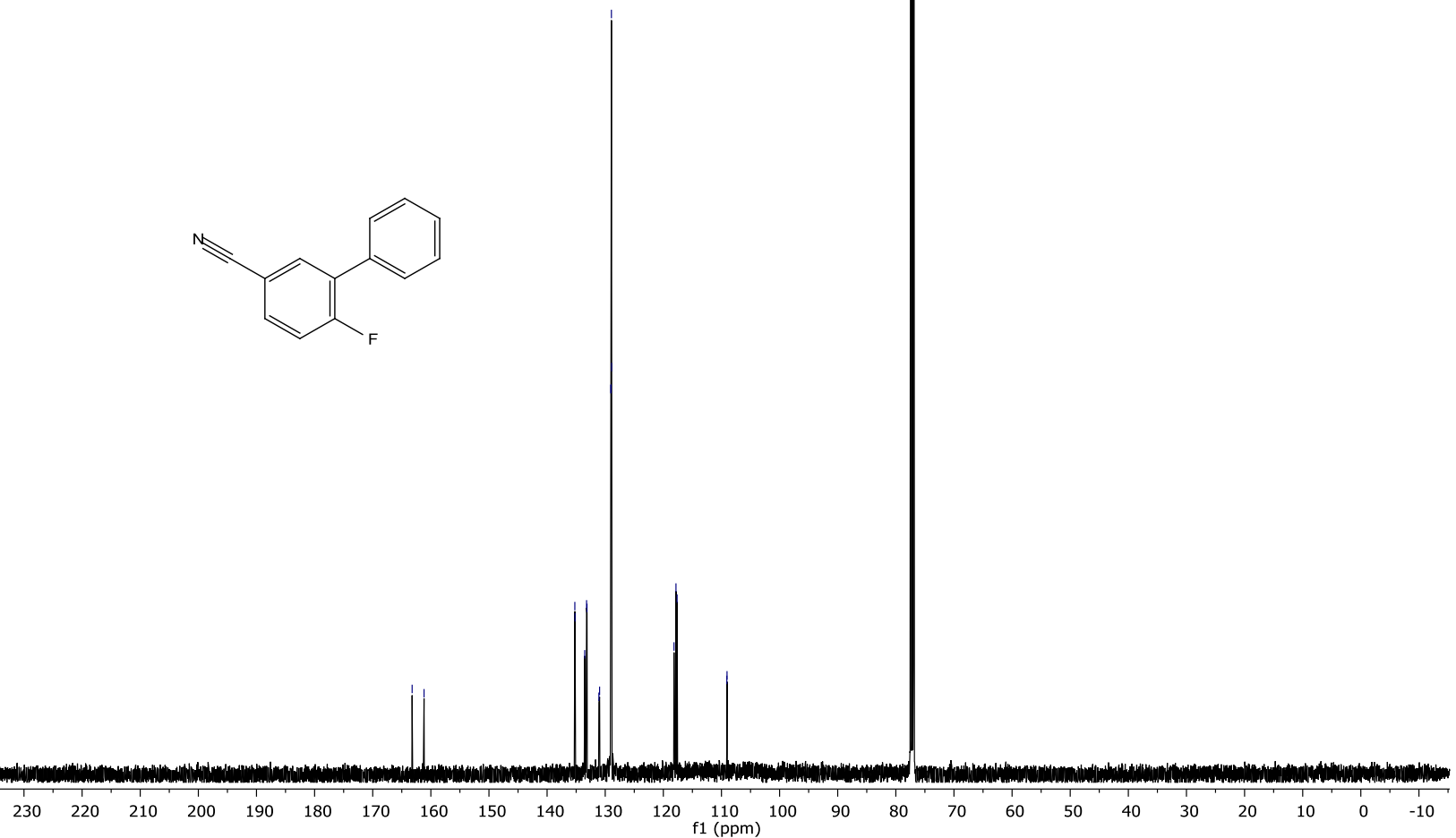

${ }^{13} \mathrm{C}\left\{{ }^{1} \mathrm{H}\right\}$ NMR $\left(101 \mathrm{MHz}, \mathrm{CDCl}_{3}\right)$ spectrum of $\mathbf{3 w}$.

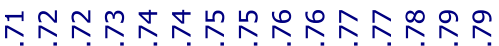

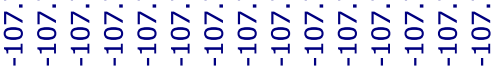
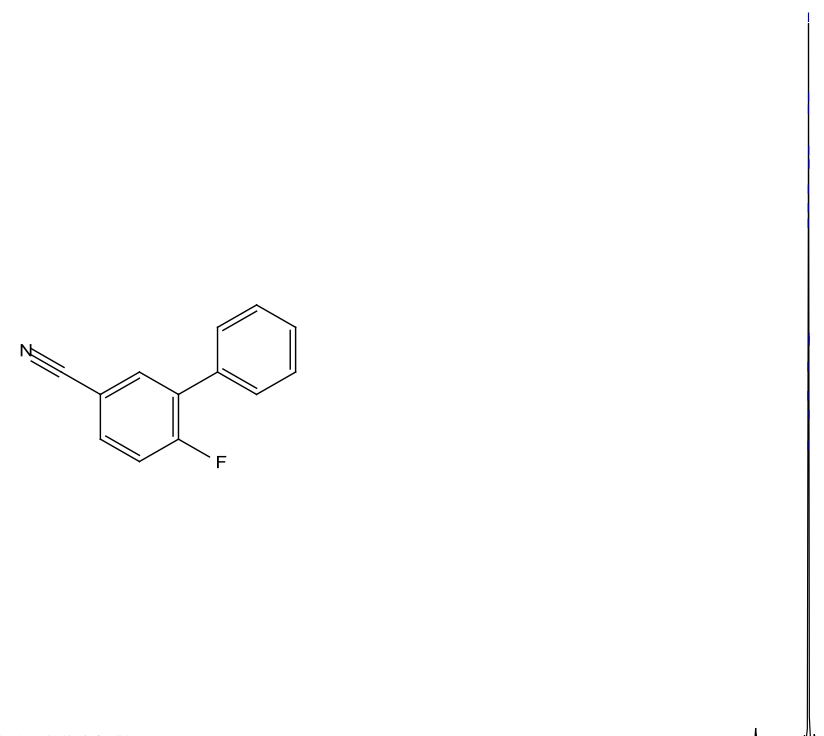

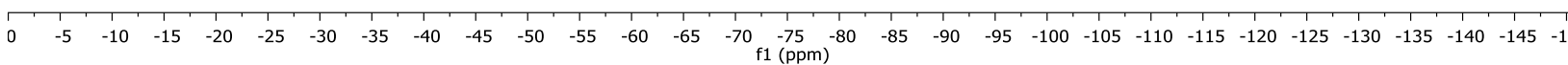

${ }^{19} \mathrm{~F}$ NMR $\left(282 \mathrm{MHz}, \mathrm{CDCl}_{3}\right)$ spectrum of $\mathbf{3 w}$. 


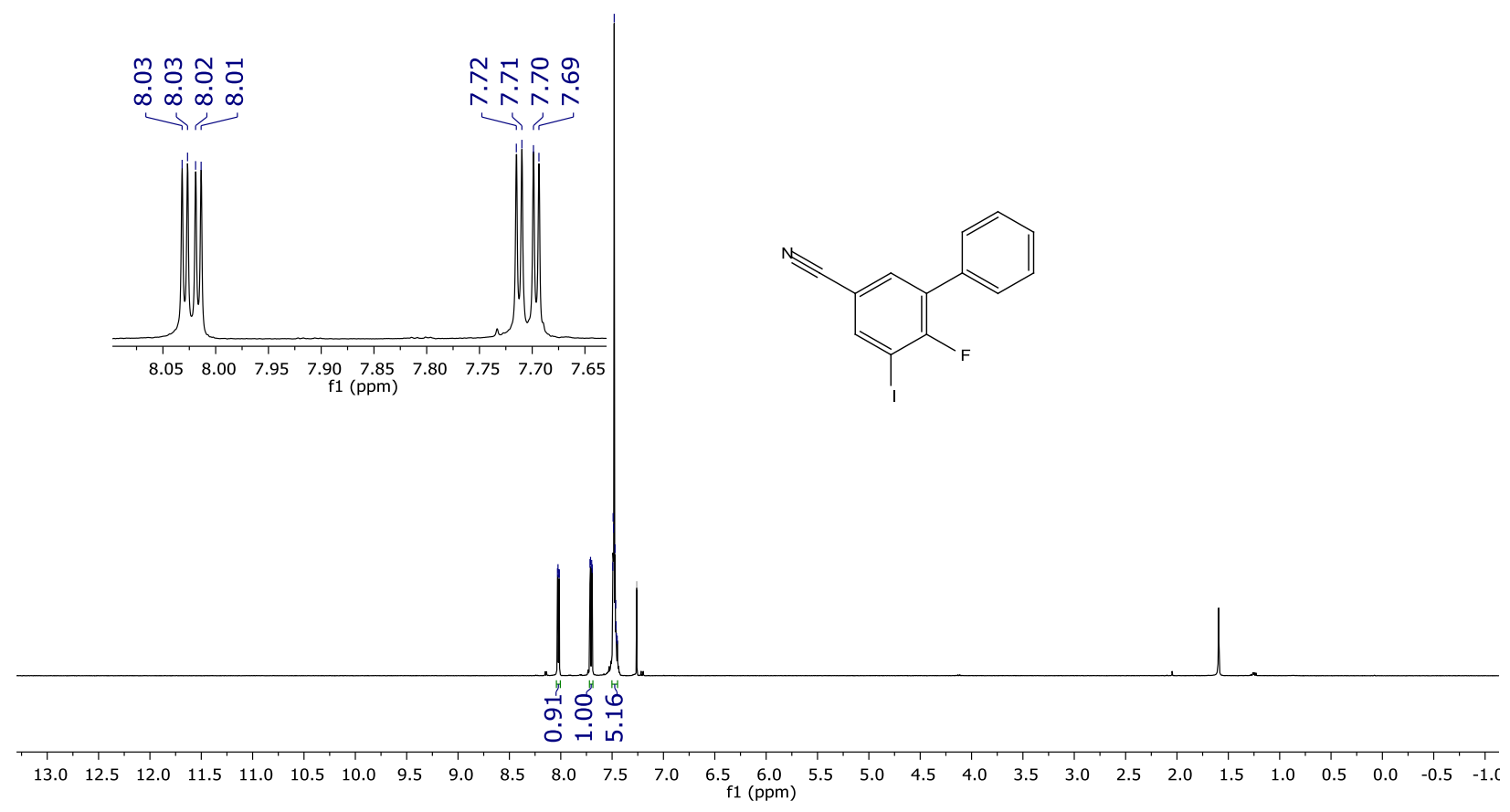

${ }^{1} \mathrm{H}$ NMR (400 MHz, $\mathrm{CDCl}_{3}$ ) spectrum of $\mathbf{4 a}$.

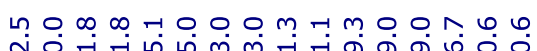
ชิं

กำ

化

$\prod_{\infty}^{\infty} \prod_{\infty}^{\infty}$

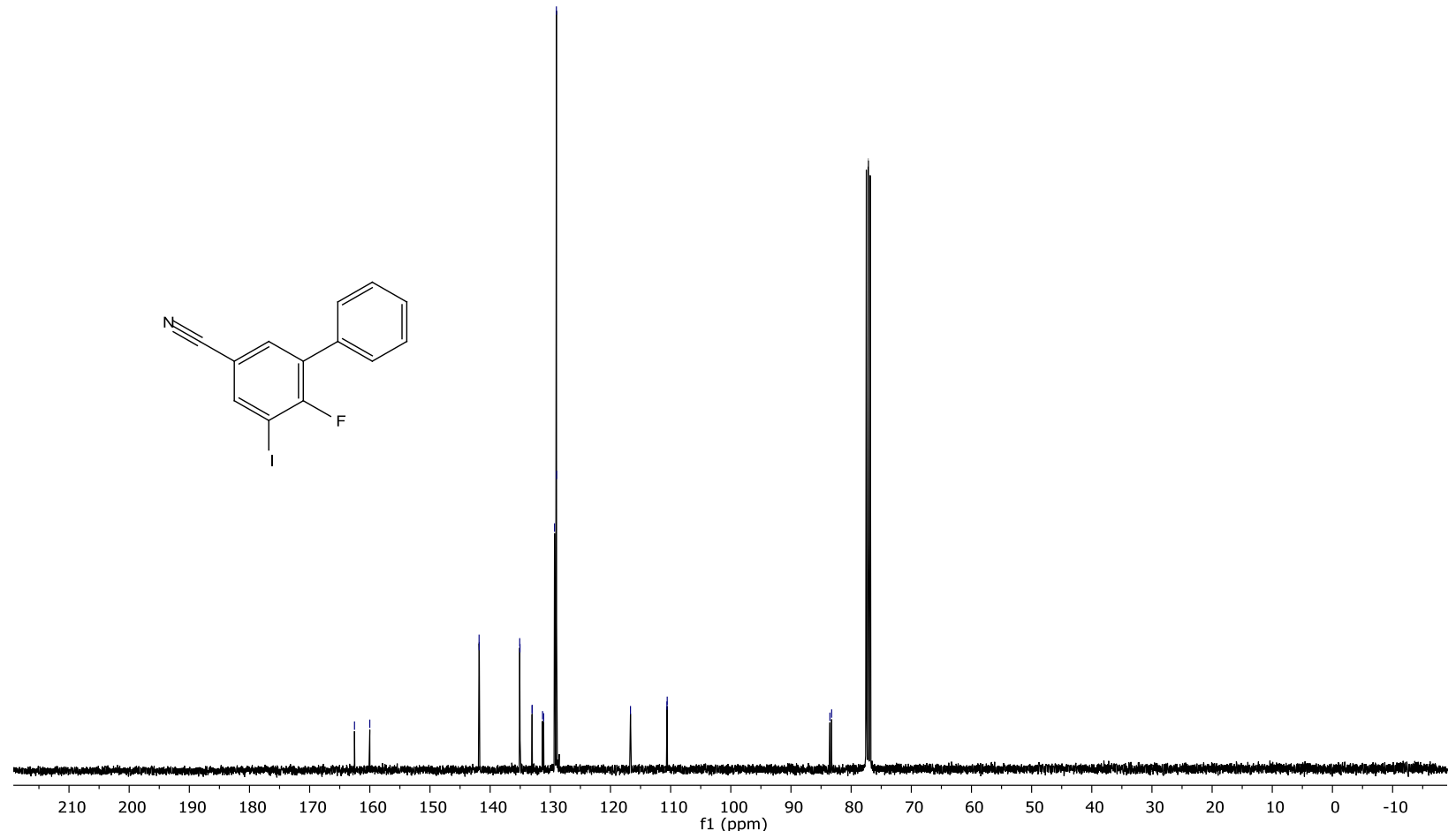

${ }^{13} \mathrm{C}\left\{{ }^{1} \mathrm{H}\right\}$ NMR $\left(101 \mathrm{MHz}, \mathrm{CDCl}_{3}\right)$ spectrum of $\mathbf{4 a}$. 


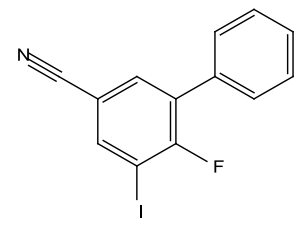

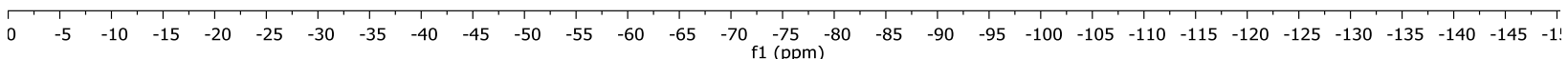

${ }^{19} \mathrm{~F}$ NMR $\left(282 \mathrm{MHz}, \mathrm{CDCl}_{3}\right)$ spectrum of $\mathbf{4 a}$.

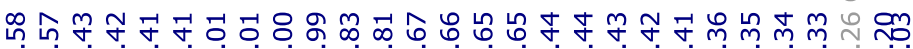

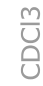

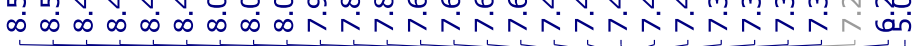

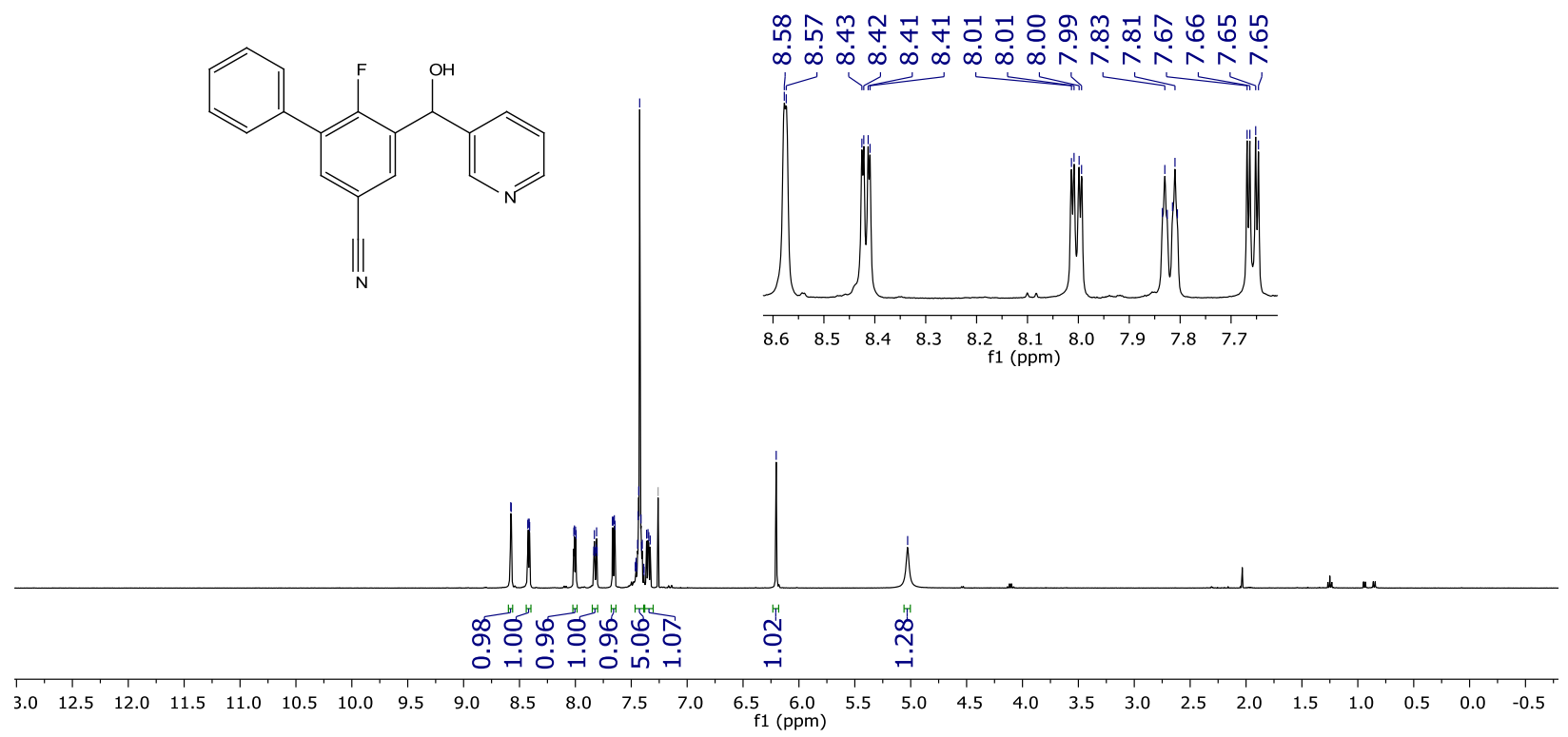

${ }^{1} \mathrm{H} \mathrm{NMR}\left(400 \mathrm{MHz}, \mathrm{CDCl}_{3}\right)$ spectrum of $\mathbf{4 b}$. 


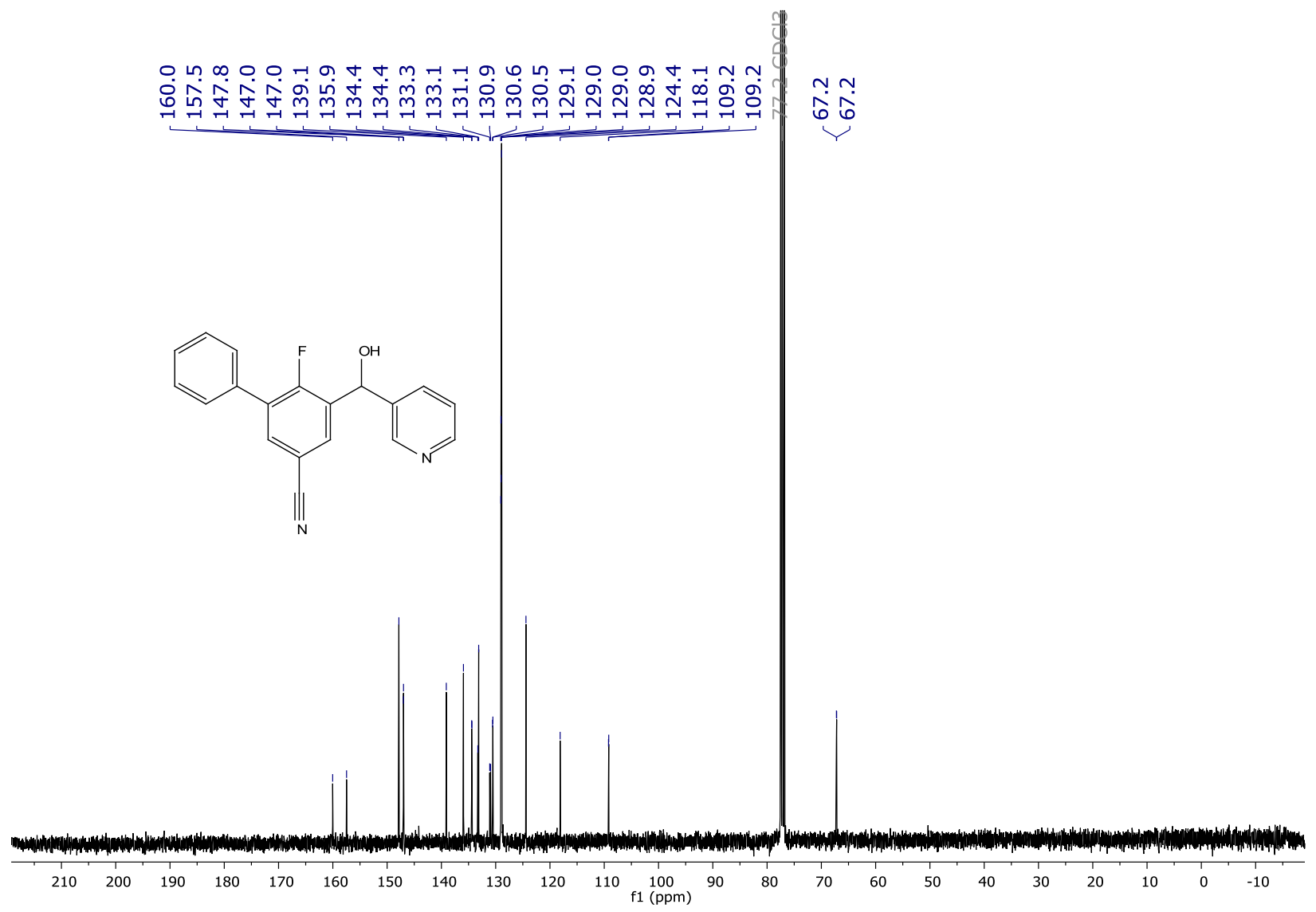

${ }^{13} \mathrm{C}\left\{{ }^{1} \mathrm{H}\right\}$ NMR $\left(101 \mathrm{MHz}, \mathrm{CDCl}_{3}\right)$ spectrum of $\mathbf{4 b}$.

$\stackrel{\infty}{\sim} \vec{q} \underset{q}{q}$

$m m_{T}^{m}$

각

$+1$

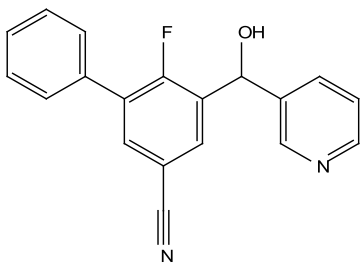

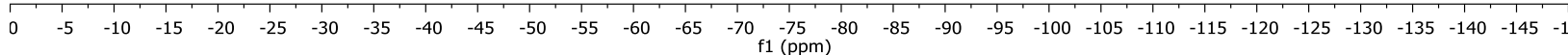

${ }^{19} \mathrm{~F}$ NMR $\left(282 \mathrm{MHz}, \mathrm{CDCl}_{3}\right)$ spectrum of $\mathbf{4 b}$. 

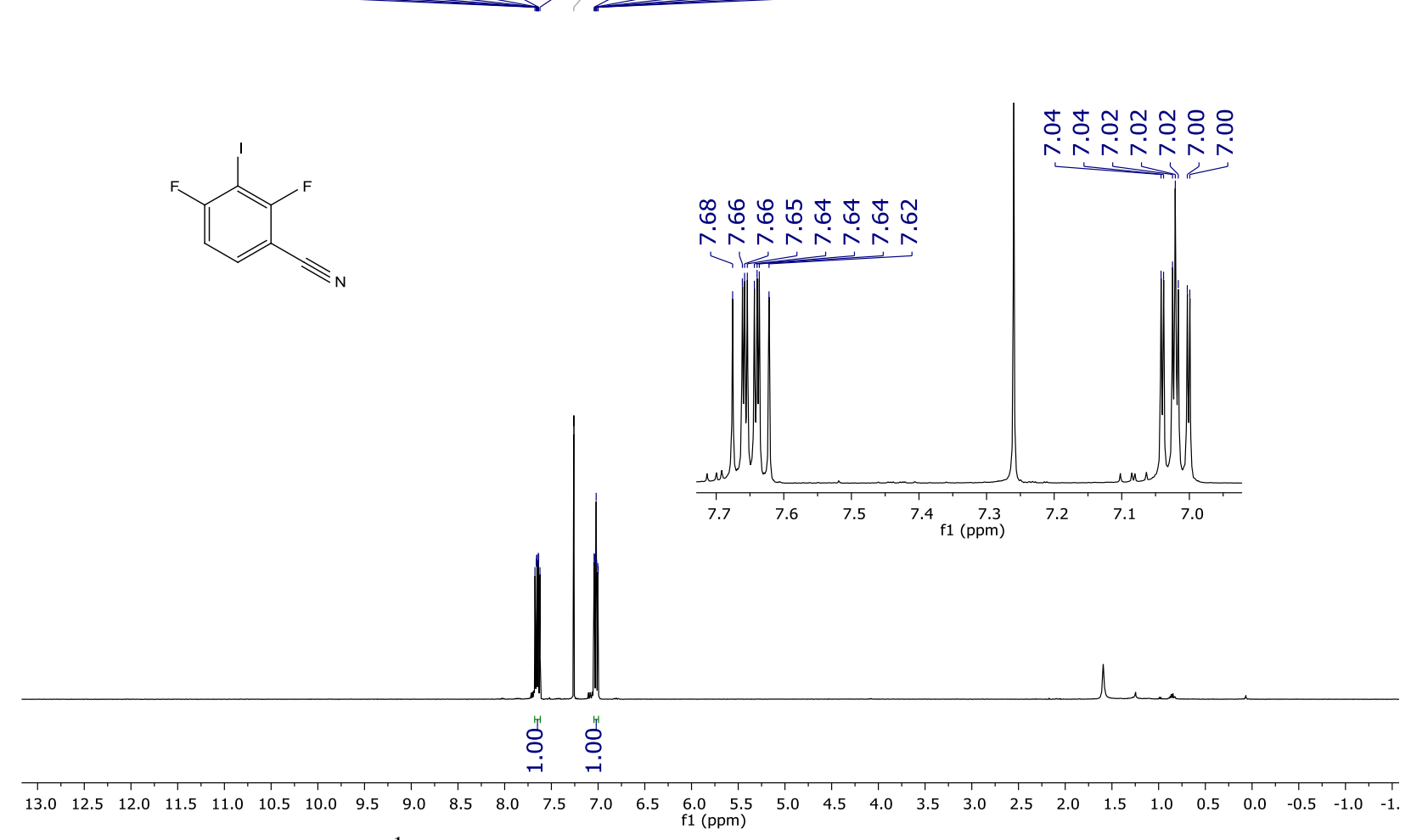

${ }^{1} \mathrm{H}$ NMR (400 MHz, $\mathrm{CDCl}_{3}$ ) spectrum of $7 \mathbf{a}$.

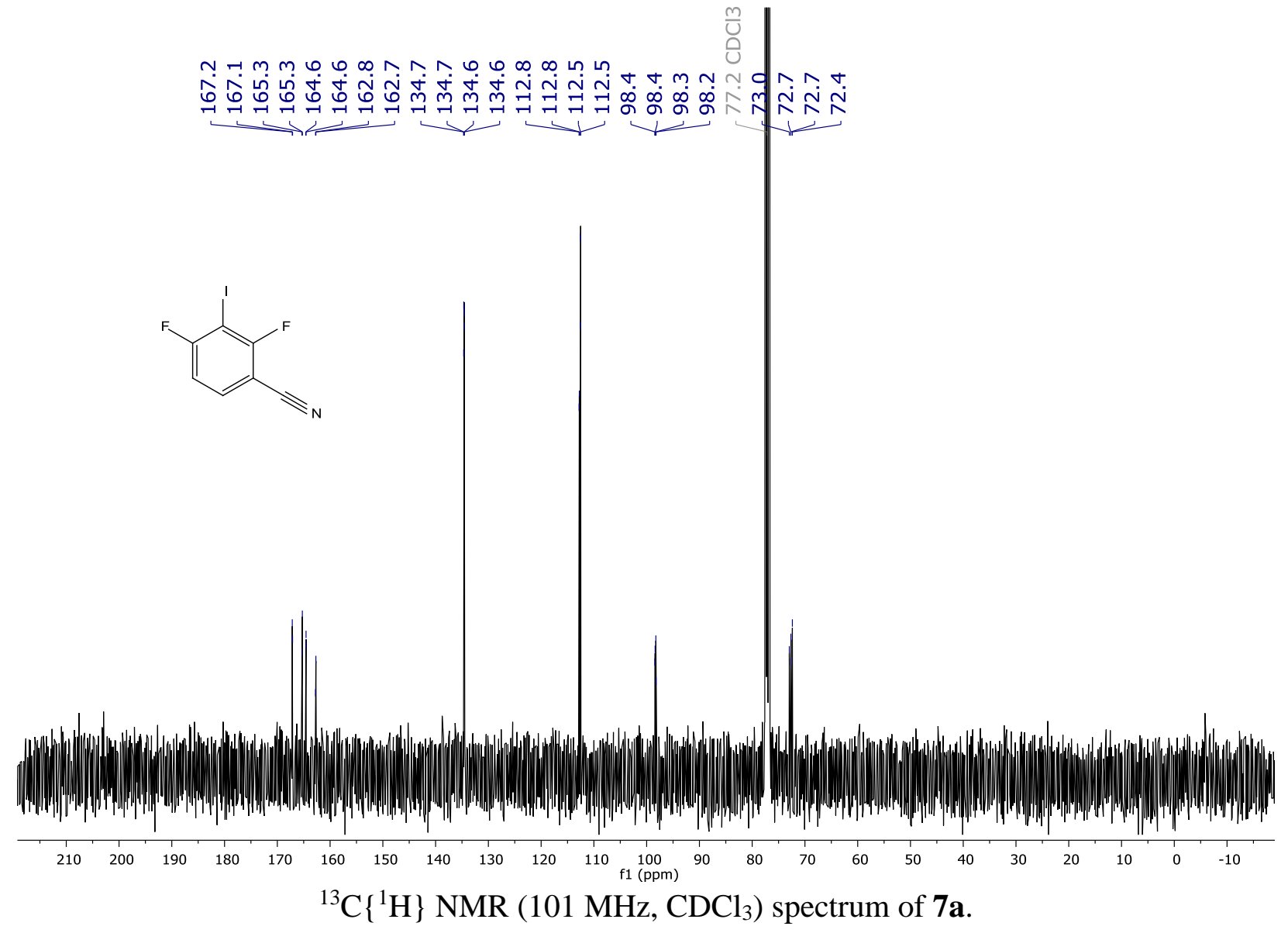



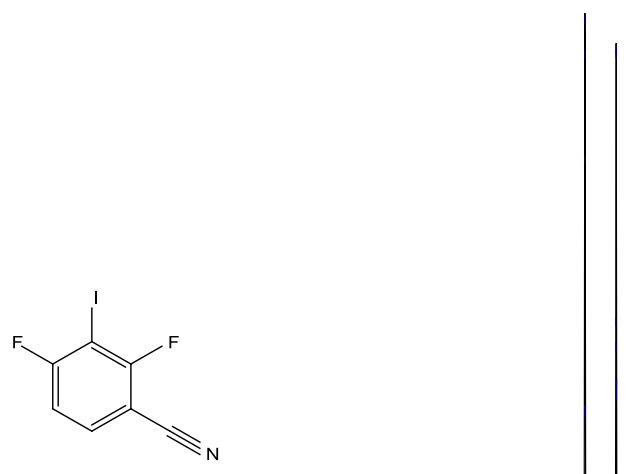

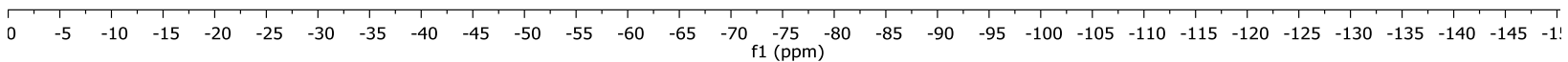

${ }^{19} \mathrm{~F} \mathrm{NMR}\left(282 \mathrm{MHz}, \mathrm{CDCl}_{3}\right)$ spectrum of $7 \mathbf{a}$.

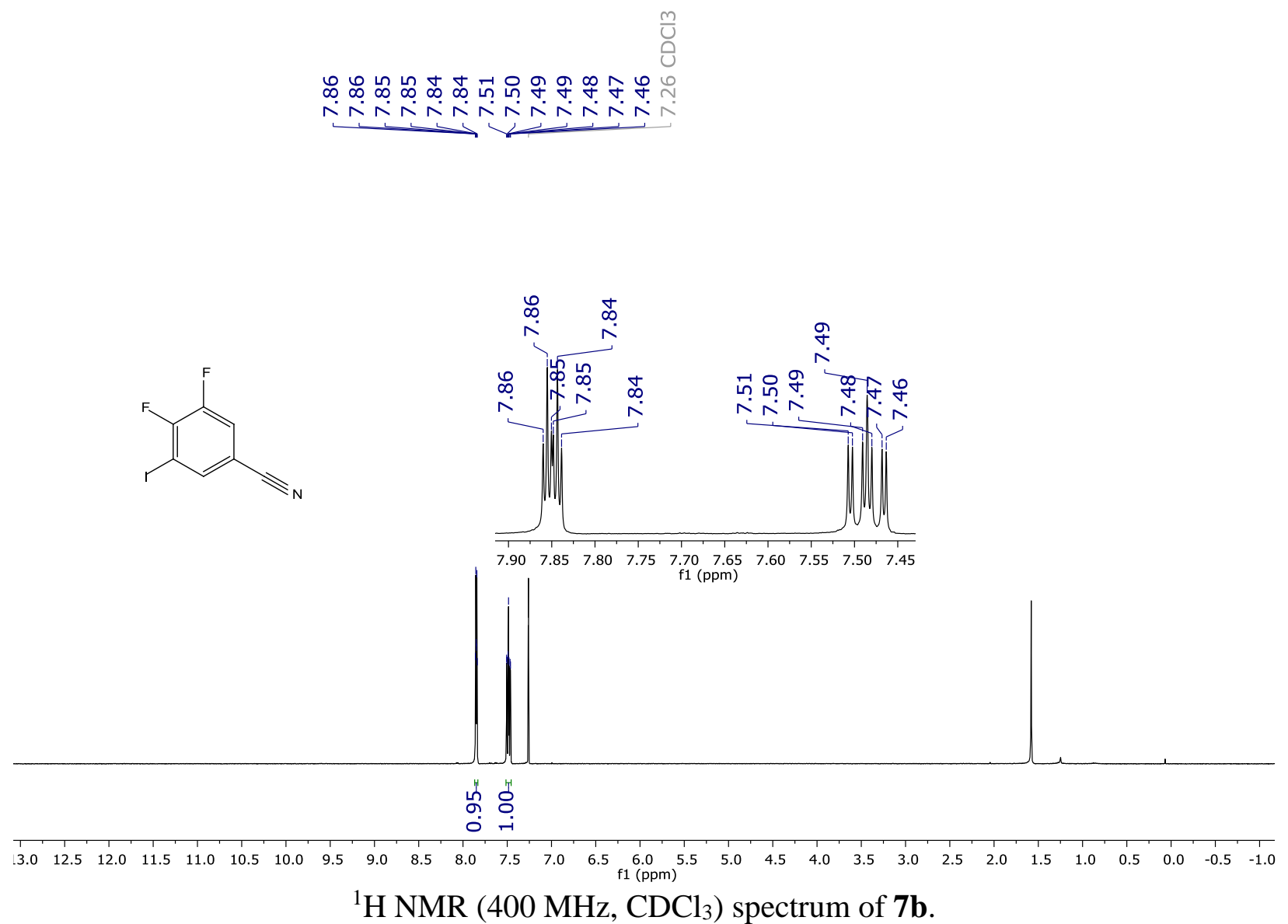




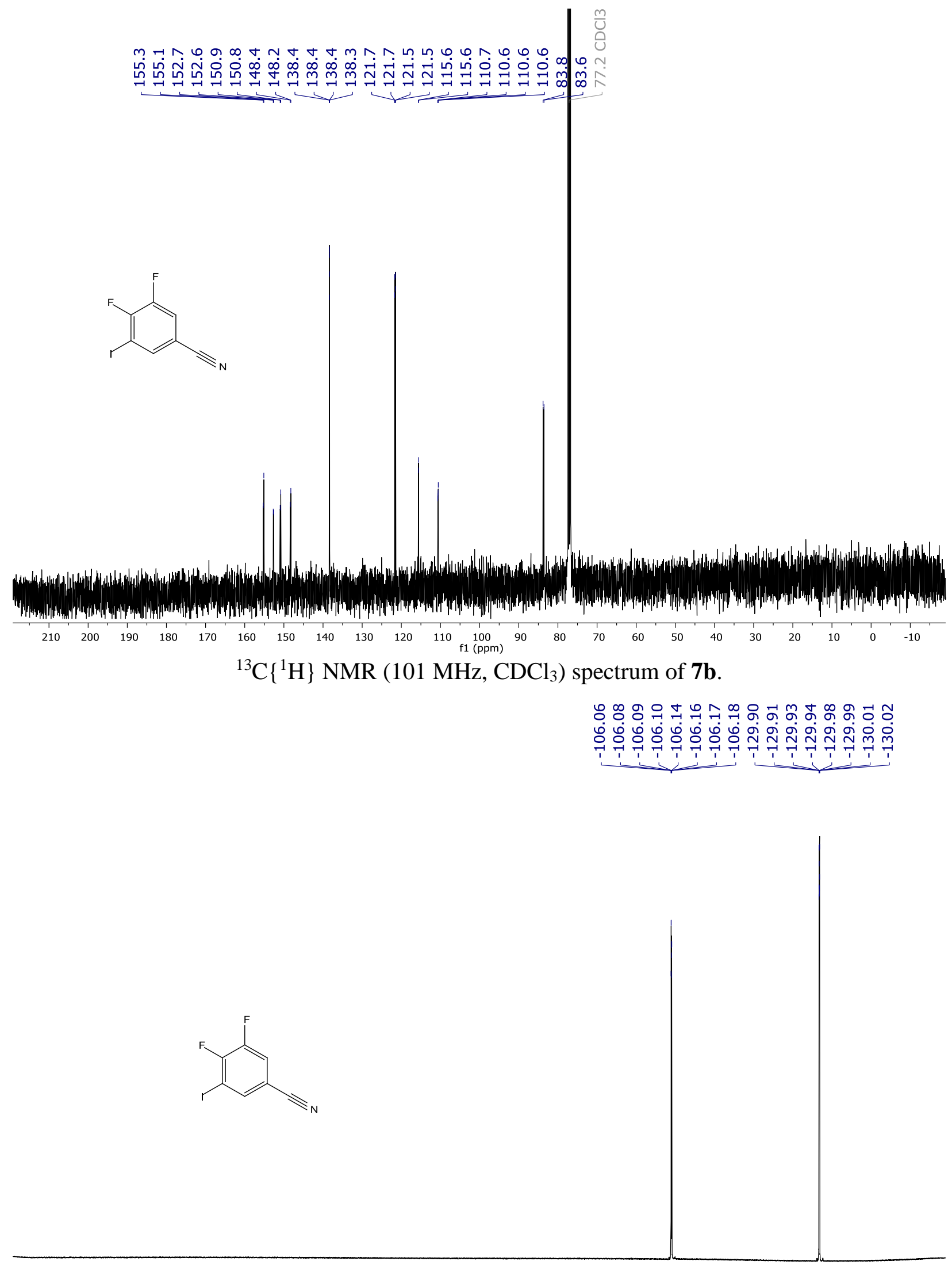

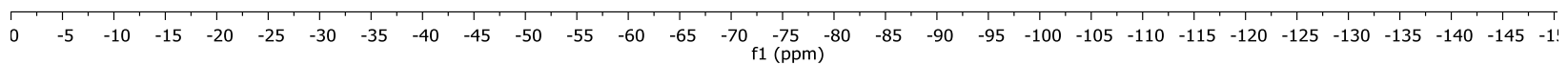

${ }^{19} \mathrm{~F}$ NMR (282 MHz, $\mathrm{CDCl}_{3}$ ) spectrum of $\mathbf{7 b}$. 

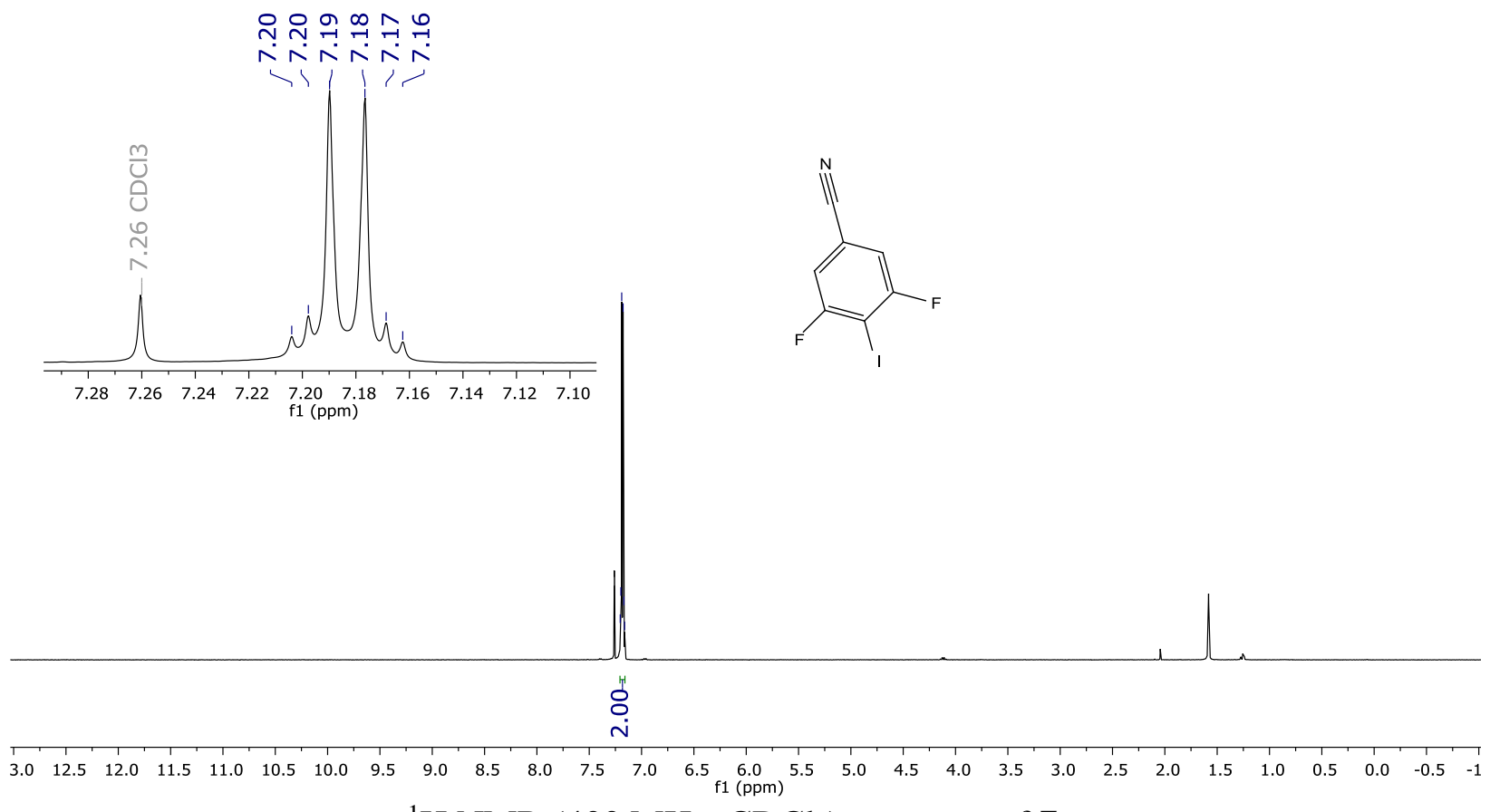

${ }^{1} \mathrm{H}$ NMR (400 MHz, $\mathrm{CDCl}_{3}$ ) spectrum of $7 \mathbf{c}$.

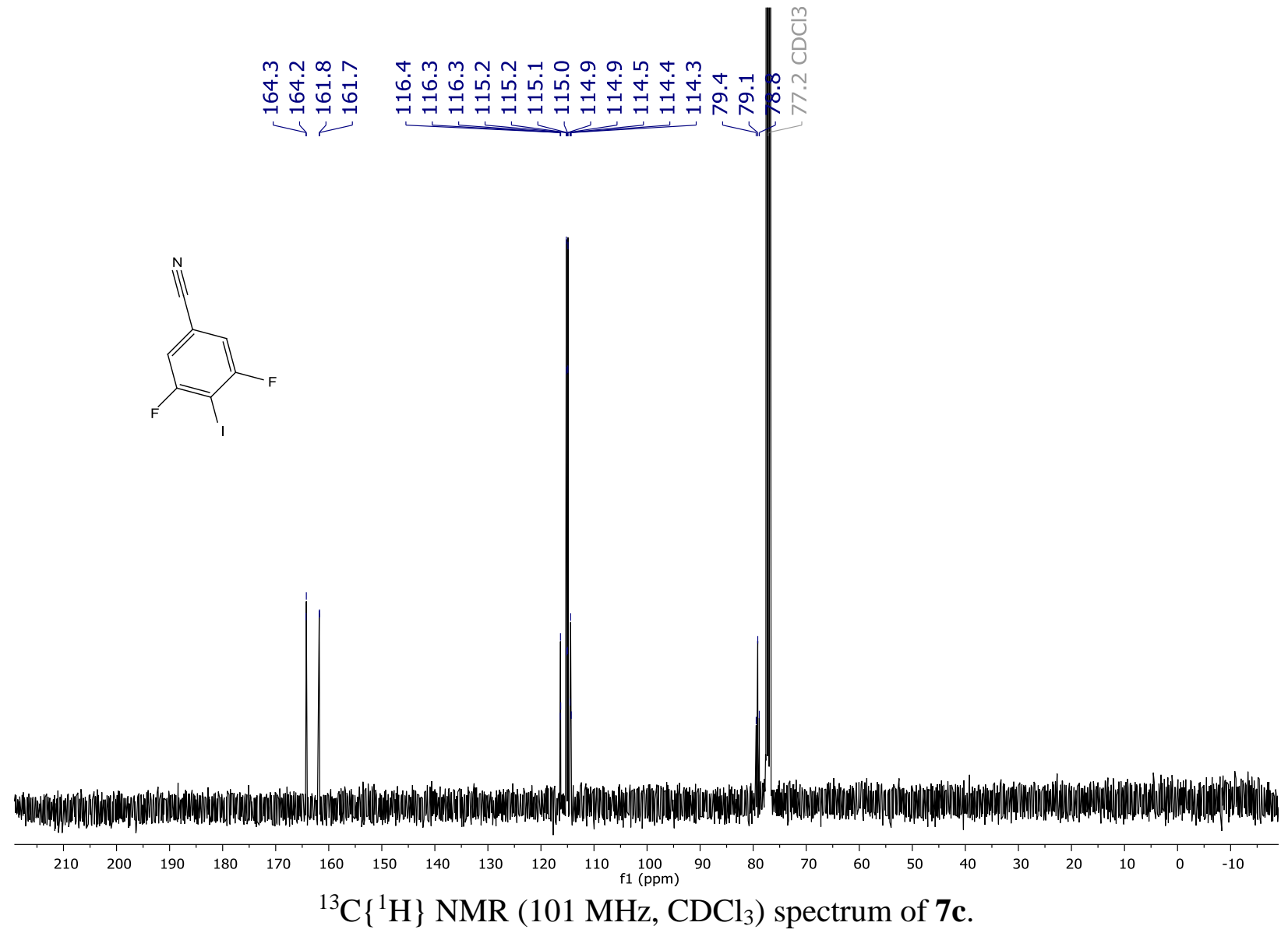



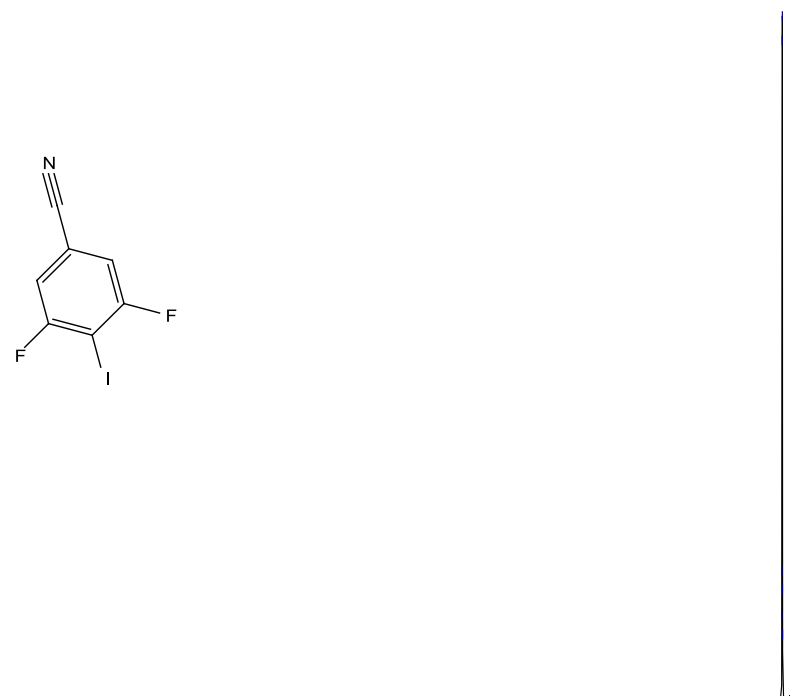

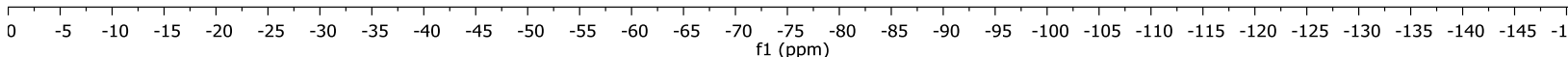

${ }^{19} \mathrm{~F}$ NMR $\left(282 \mathrm{MHz}, \mathrm{CDCl}_{3}\right)$ spectrum of $7 \mathbf{c}$.

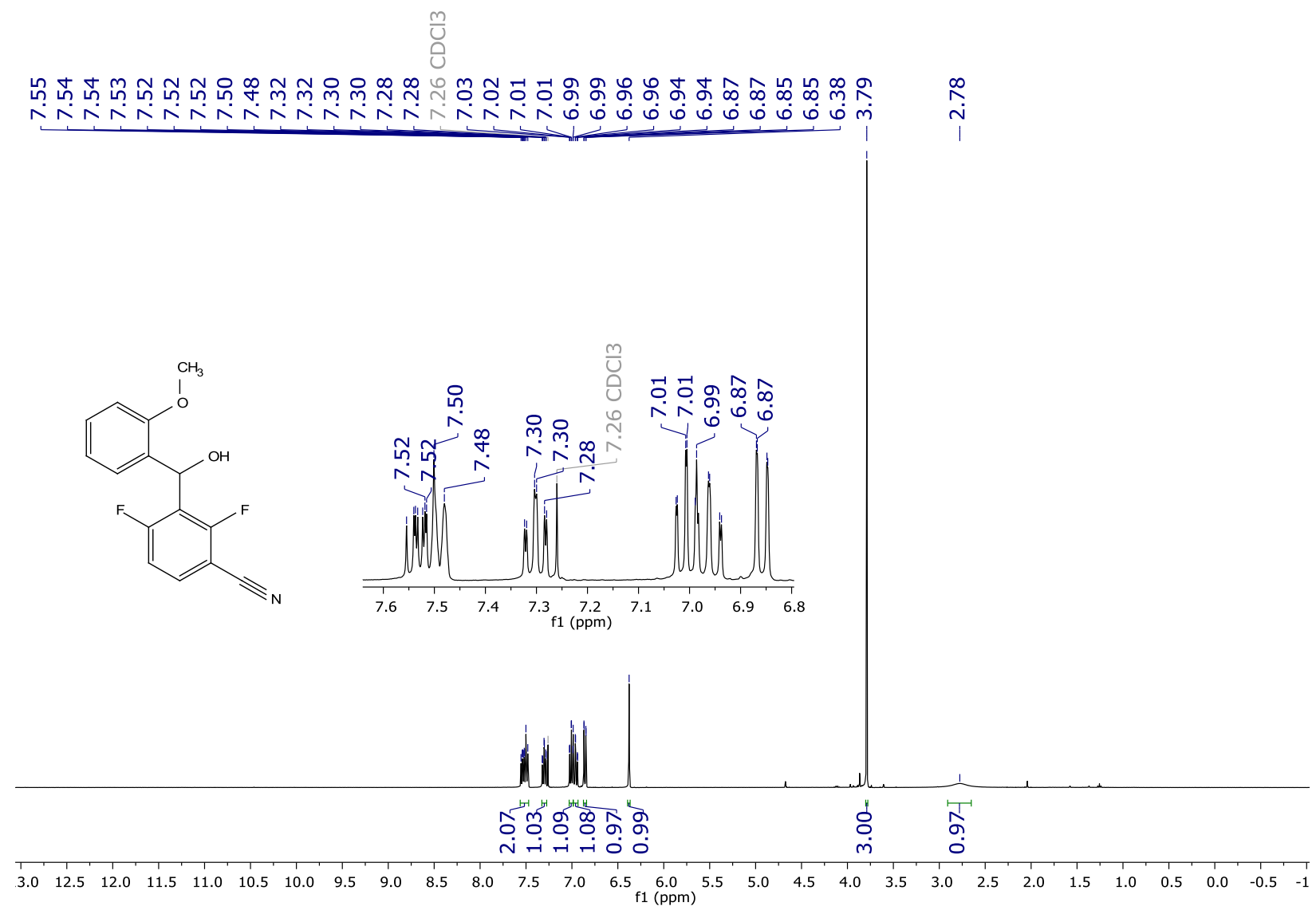

${ }^{1} \mathrm{H}$ NMR $\left(400 \mathrm{MHz}, \mathrm{CDCl}_{3}\right)$ spectrum of $\mathbf{7 d}$. 


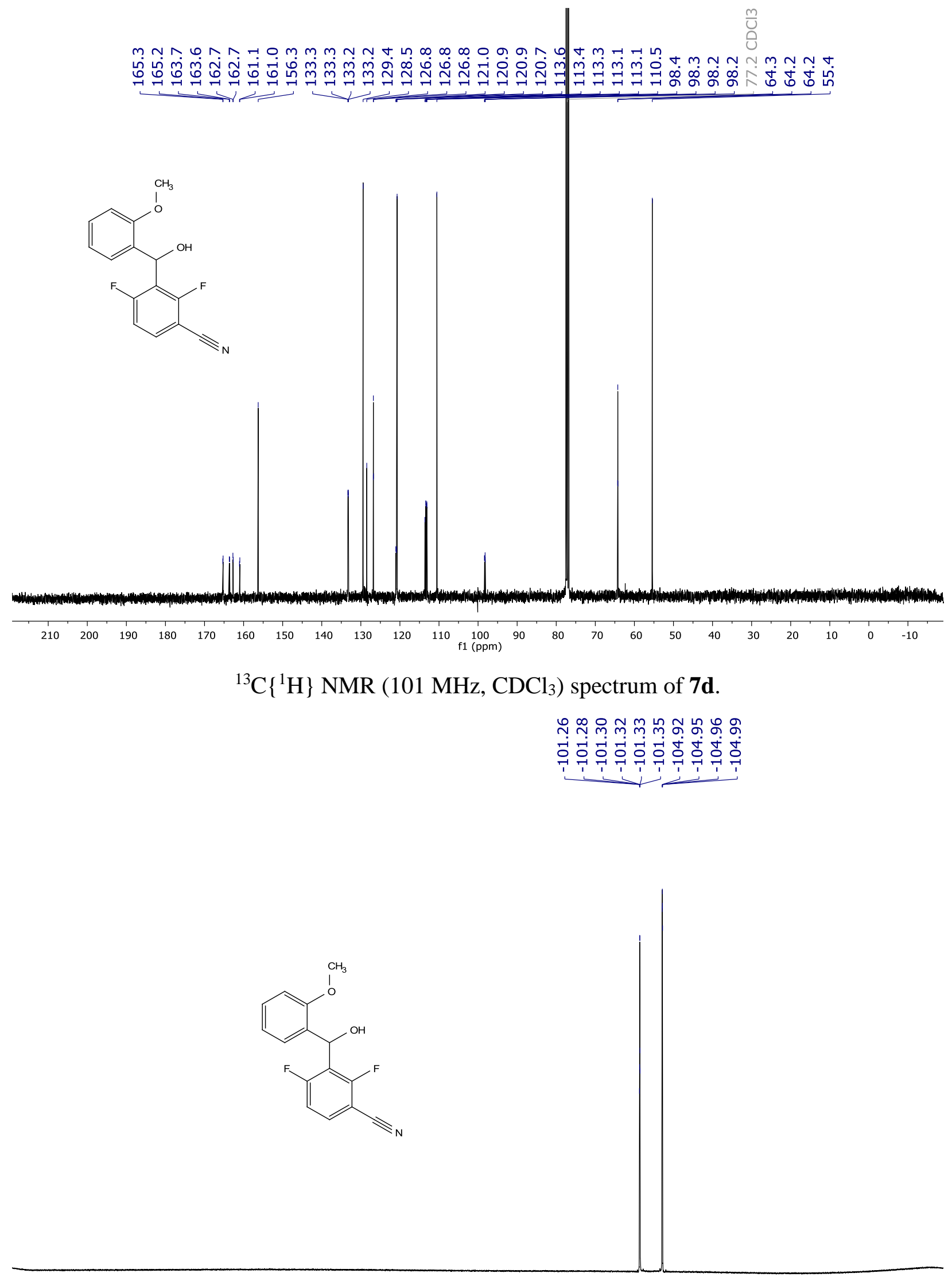

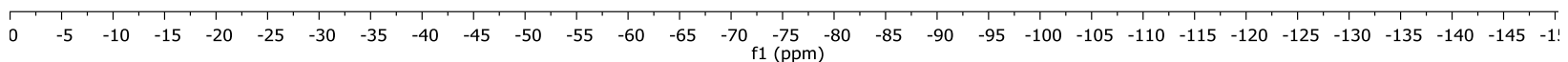

${ }^{19} \mathrm{~F}$ NMR (282 MHz, $\left.\mathrm{CDCl}_{3}\right)$ spectrum of $\mathbf{7 d}$. 


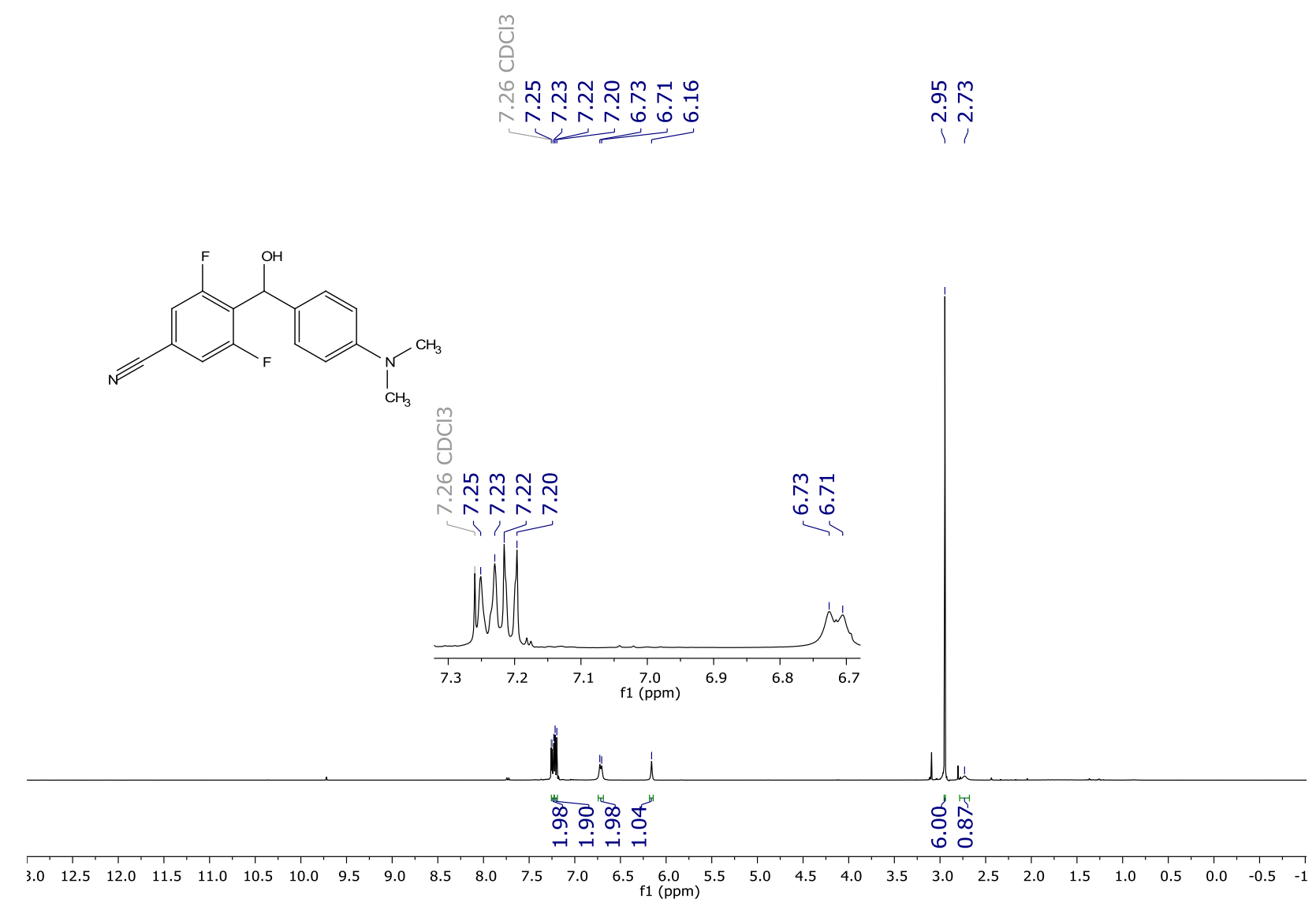

${ }^{1} \mathrm{H}$ NMR (400 MHz, $\mathrm{CDCl}_{3}$ ) spectrum of $\mathbf{7 e}$.

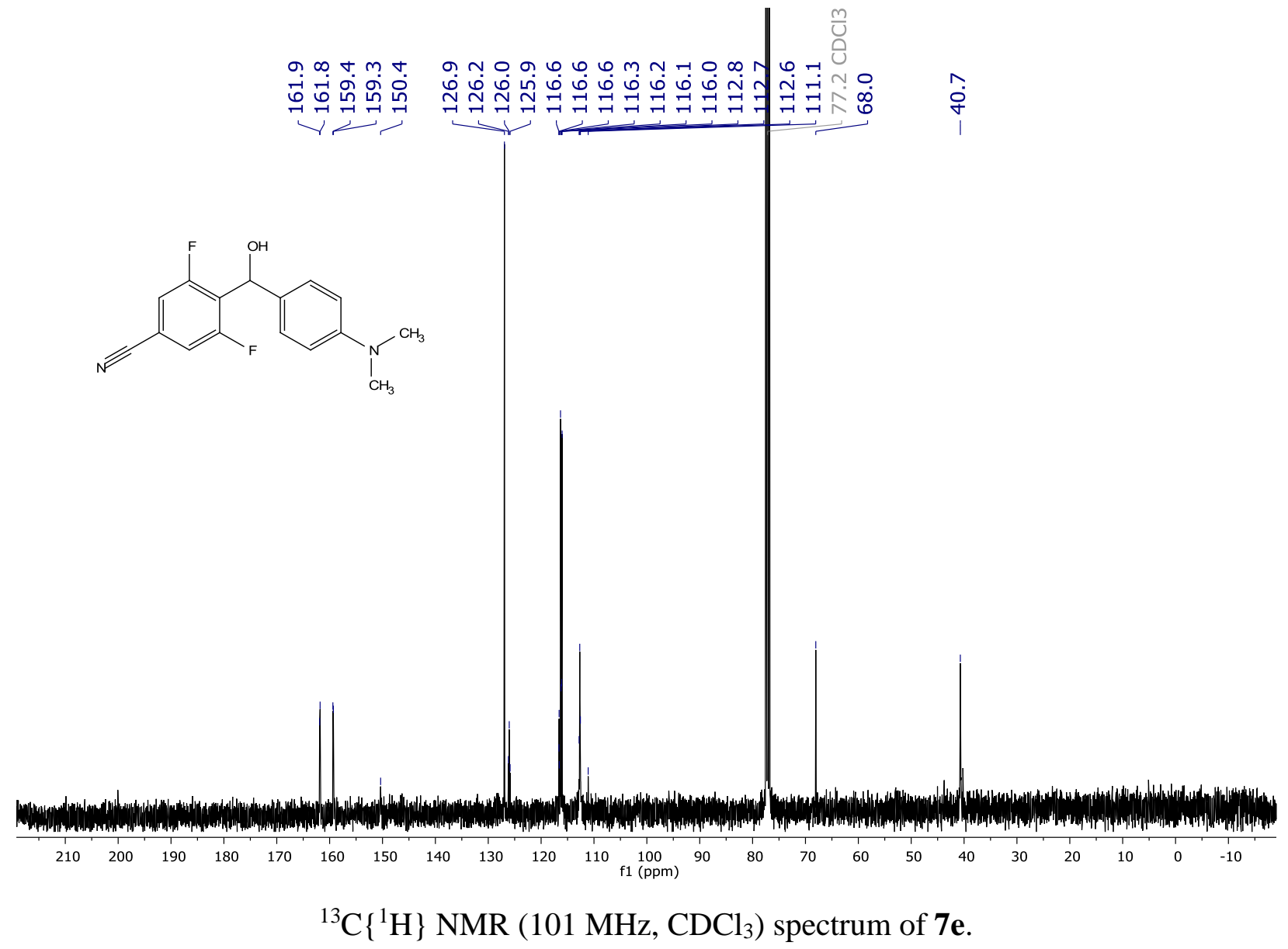



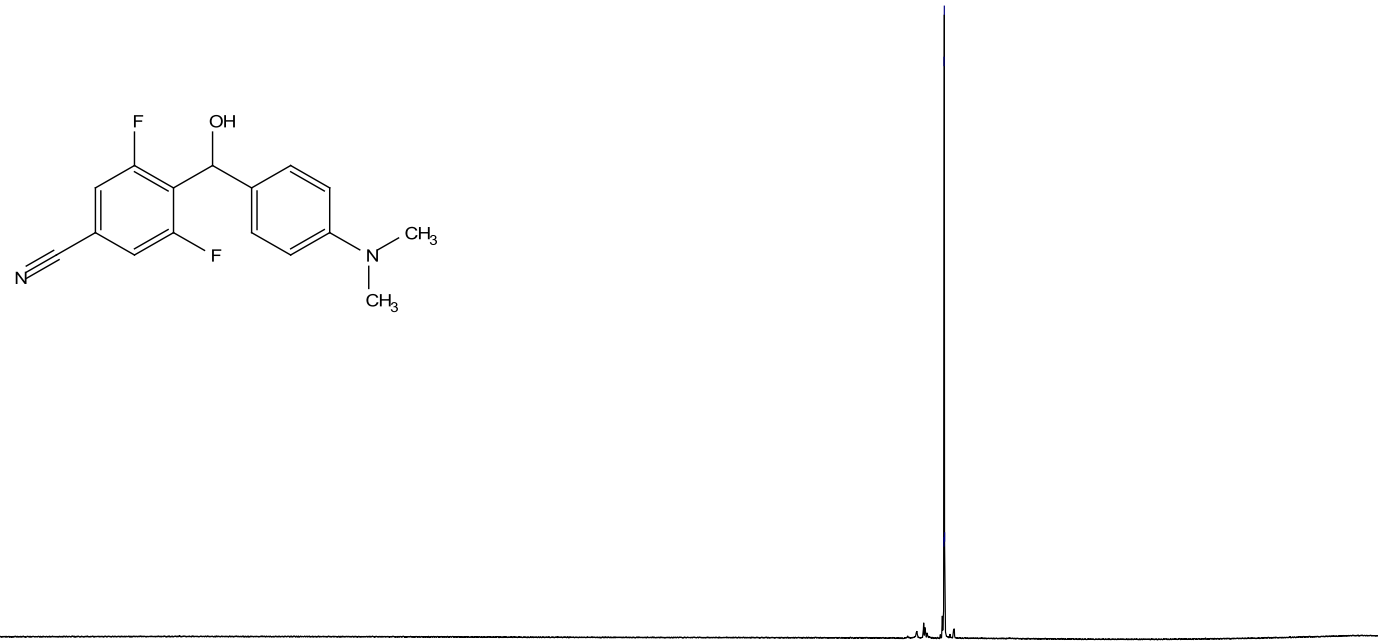

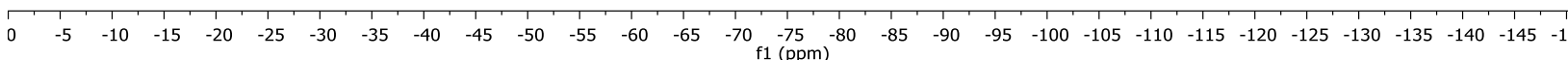

${ }^{19} \mathrm{~F}$ NMR $\left(282 \mathrm{MHz}, \mathrm{CDCl}_{3}\right)$ spectrum of $7 \mathbf{e}$.

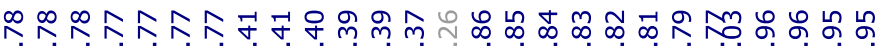

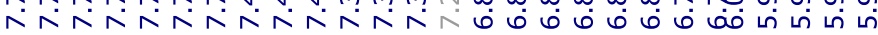
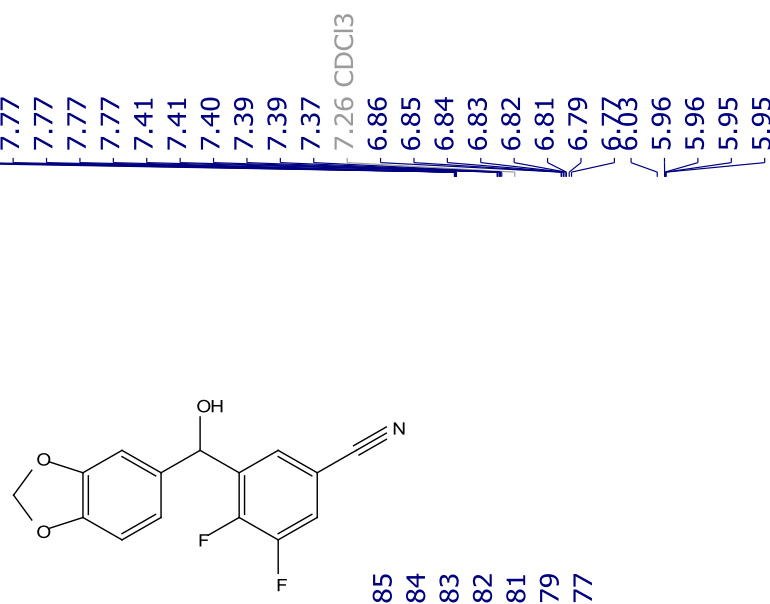

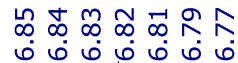
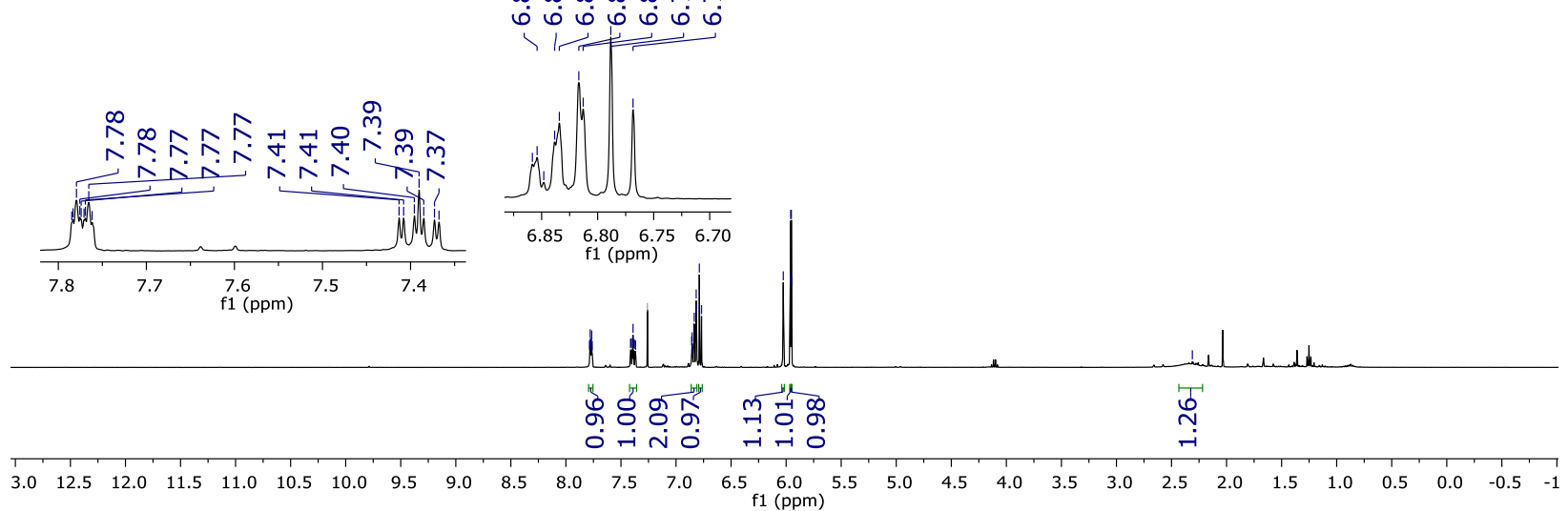

${ }^{1} \mathrm{H}$ NMR $\left(400 \mathrm{MHz}, \mathrm{CDCl}_{3}\right)$ spectrum of $\mathbf{7 f}$. 


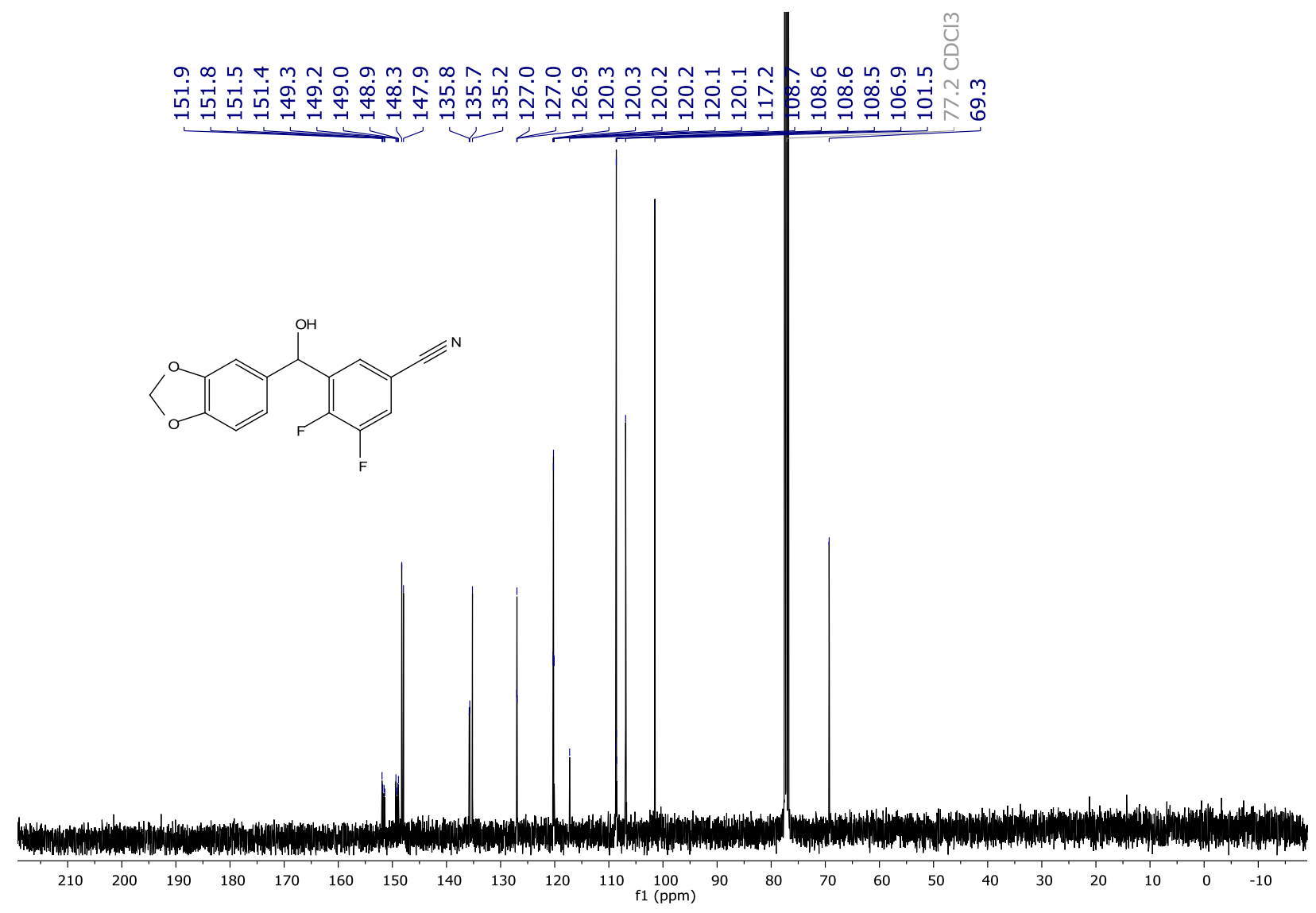

${ }^{13} \mathrm{C}\left\{{ }^{1} \mathrm{H}\right\} \mathrm{NMR}\left(101 \mathrm{MHz}, \mathrm{CDCl}_{3}\right)$ spectrum of $\mathbf{7 f}$.

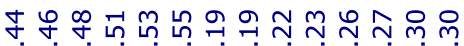

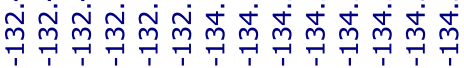

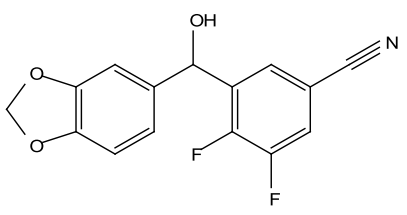

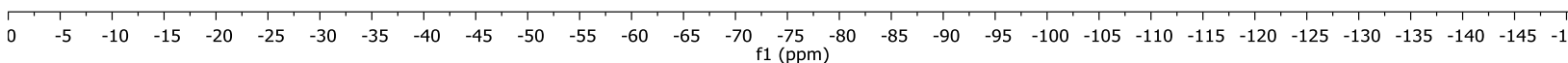

${ }^{19} \mathrm{~F}$ NMR $\left(282 \mathrm{MHz}, \mathrm{CDCl}_{3}\right)$ spectrum of $\mathbf{7 f}$. 

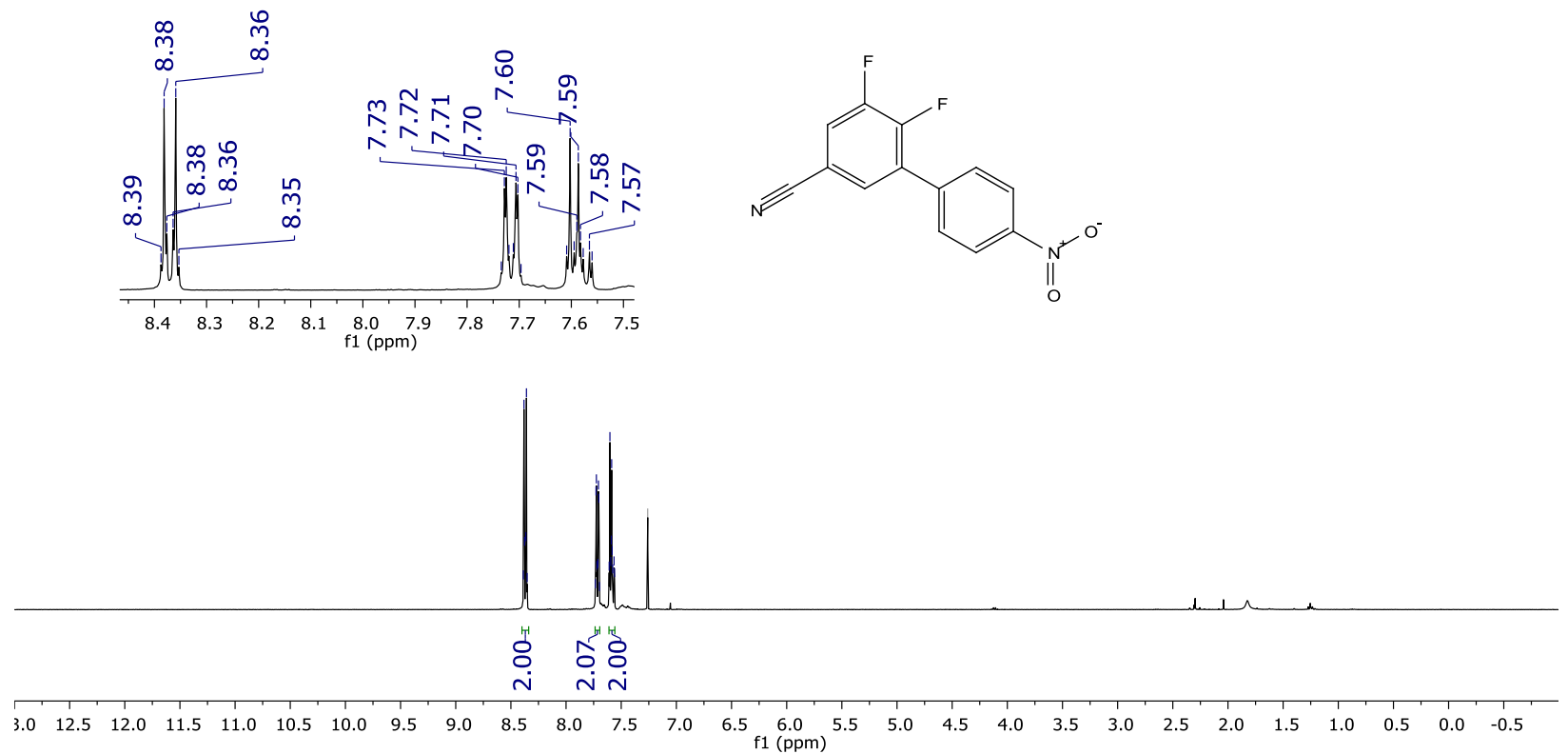

${ }^{1} \mathrm{H}$ NMR (400 MHz, $\left.\mathrm{CDCl}_{3}\right)$ spectrum of $\mathbf{7 g}$.

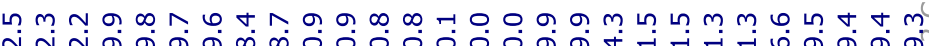

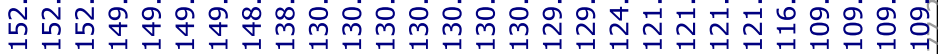
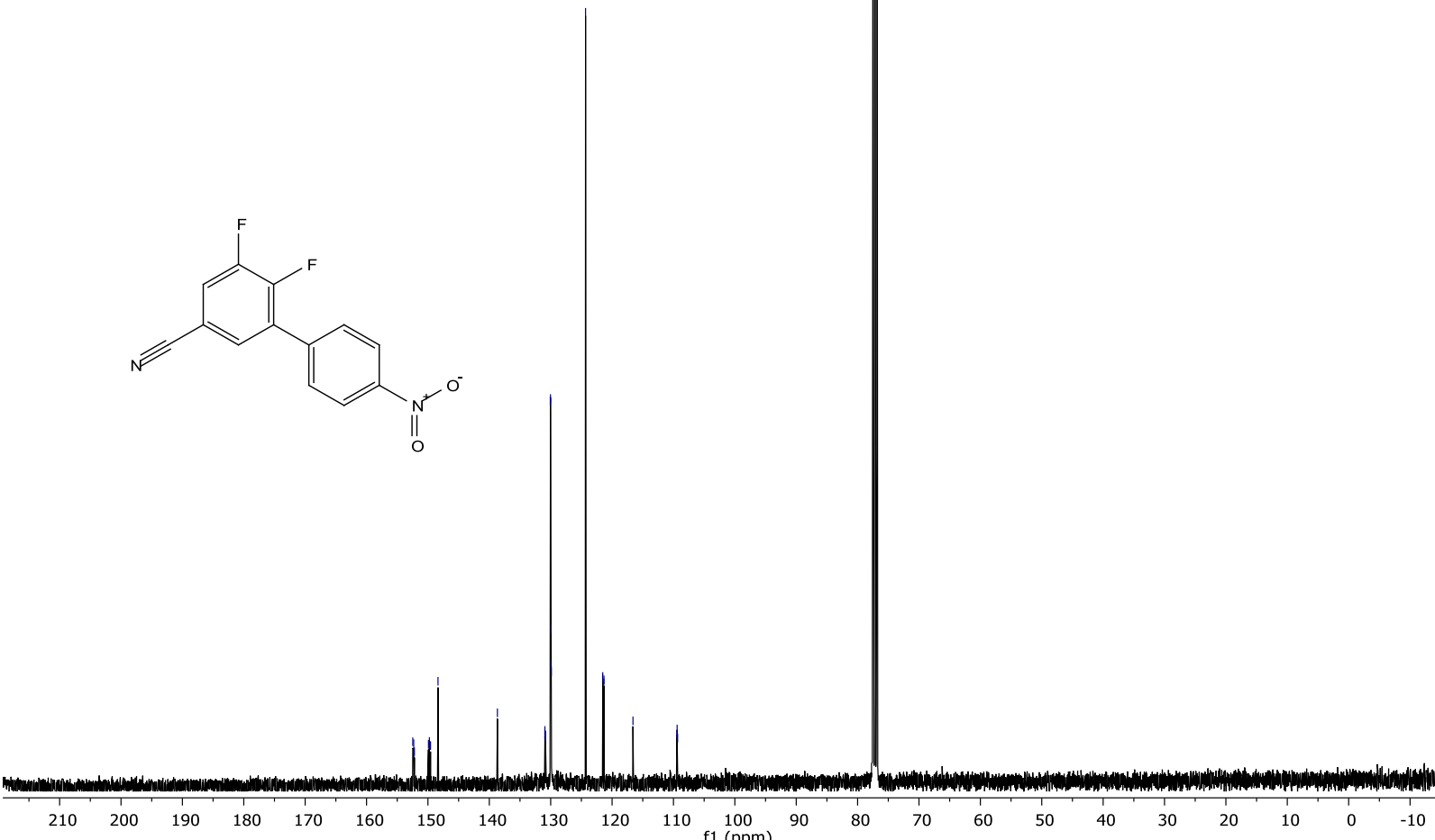

${ }^{13} \mathrm{C}\left\{{ }^{1} \mathrm{H}\right\}$ NMR $\left(101 \mathrm{MHz}, \mathrm{CDCl}_{3}\right)$ spectrum of $\mathbf{7 g}$. 


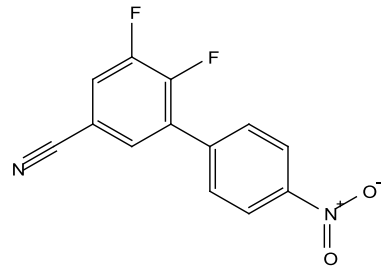

\begin{tabular}{lllllllllllllllllllllllllllllllllllllllllllllllllllllll}
\hline 0 & -5 & -10 & -15 & -20 & -25 & -30 & -35 & -40 & -45 & -50 & -55 & -60 & -65 & -70 & -75 & -80 & -85 & -90 & -95 & -100 & -105 & -110 & -115 & -120 & -125 & -130 & -135 & -140 & -145 & -1
\end{tabular}

${ }^{19} \mathrm{~F}$ NMR (282 $\left.\mathrm{MHz}, \mathrm{CDCl}_{3}\right)$ spectrum of $\mathbf{7 g}$.

怘 م

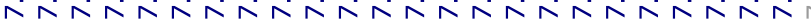

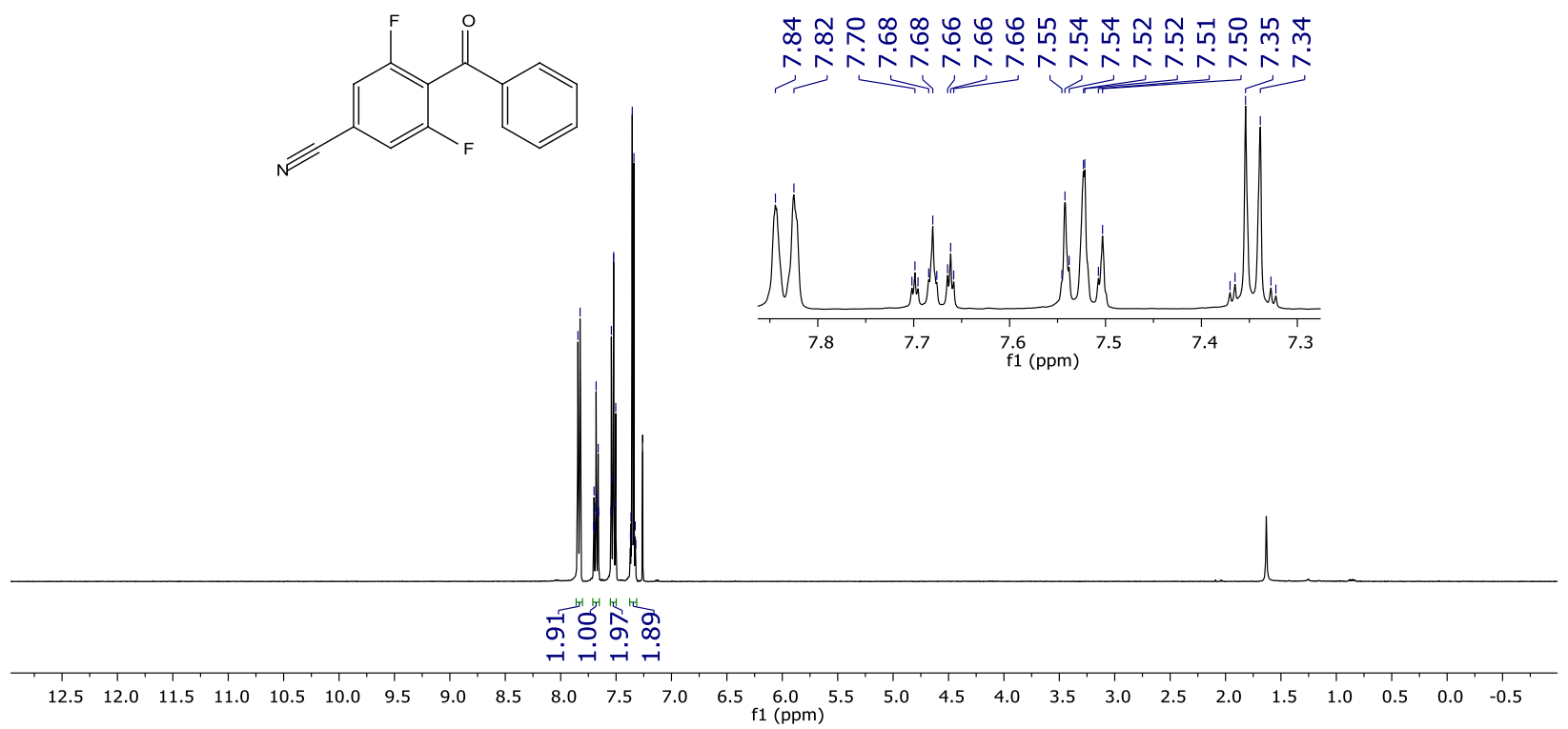

${ }^{1} \mathrm{H}$ NMR (400 MHz, $\left.\mathrm{CDCl}_{3}\right)$ spectrum of $\mathbf{7 h}$. 


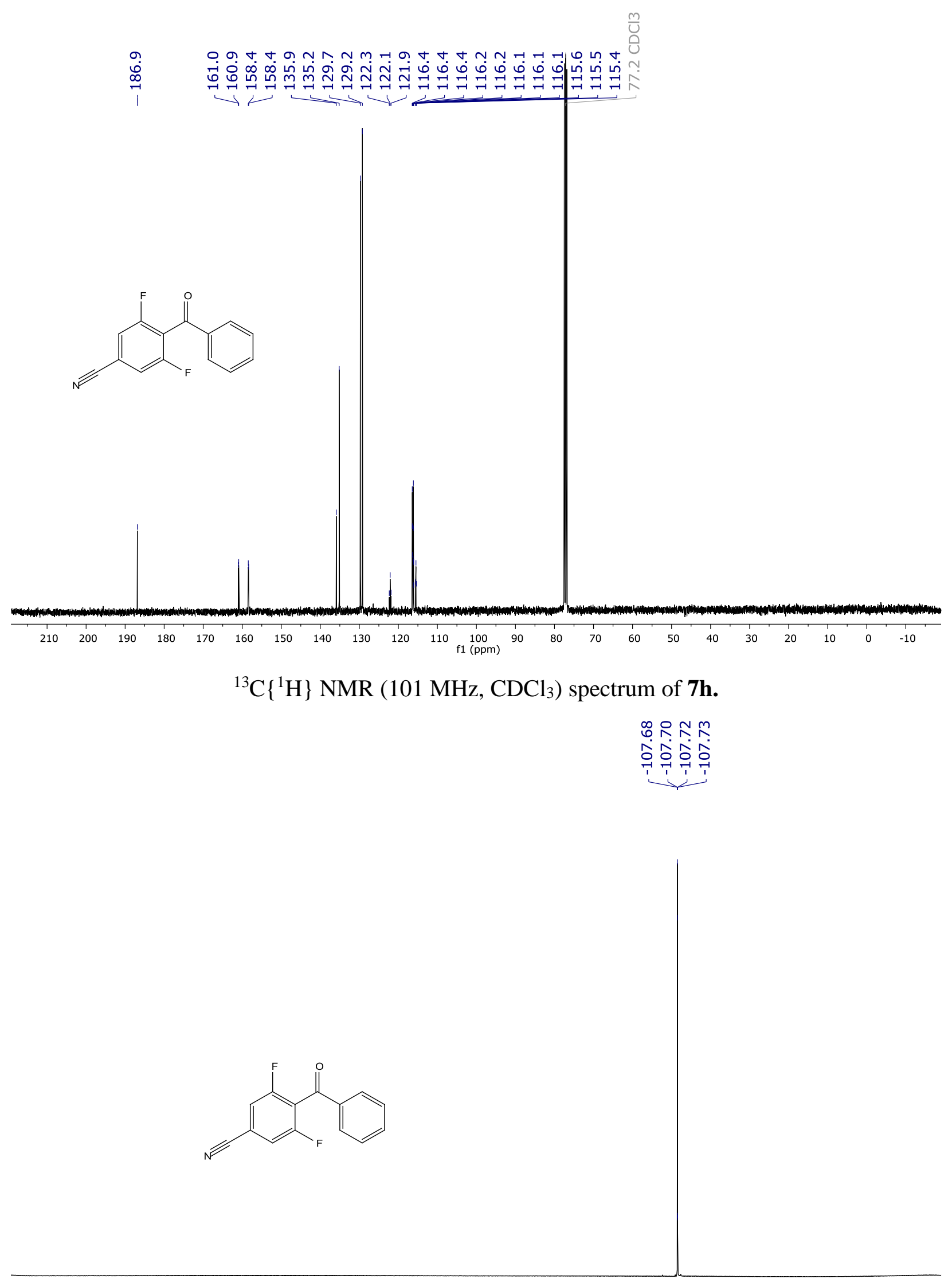

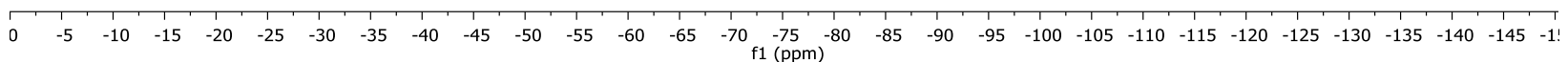

${ }^{19} \mathrm{~F}$ NMR (282 MHz, $\mathrm{CDCl}_{3}$ ) spectrum of $\mathbf{7 h}$. 


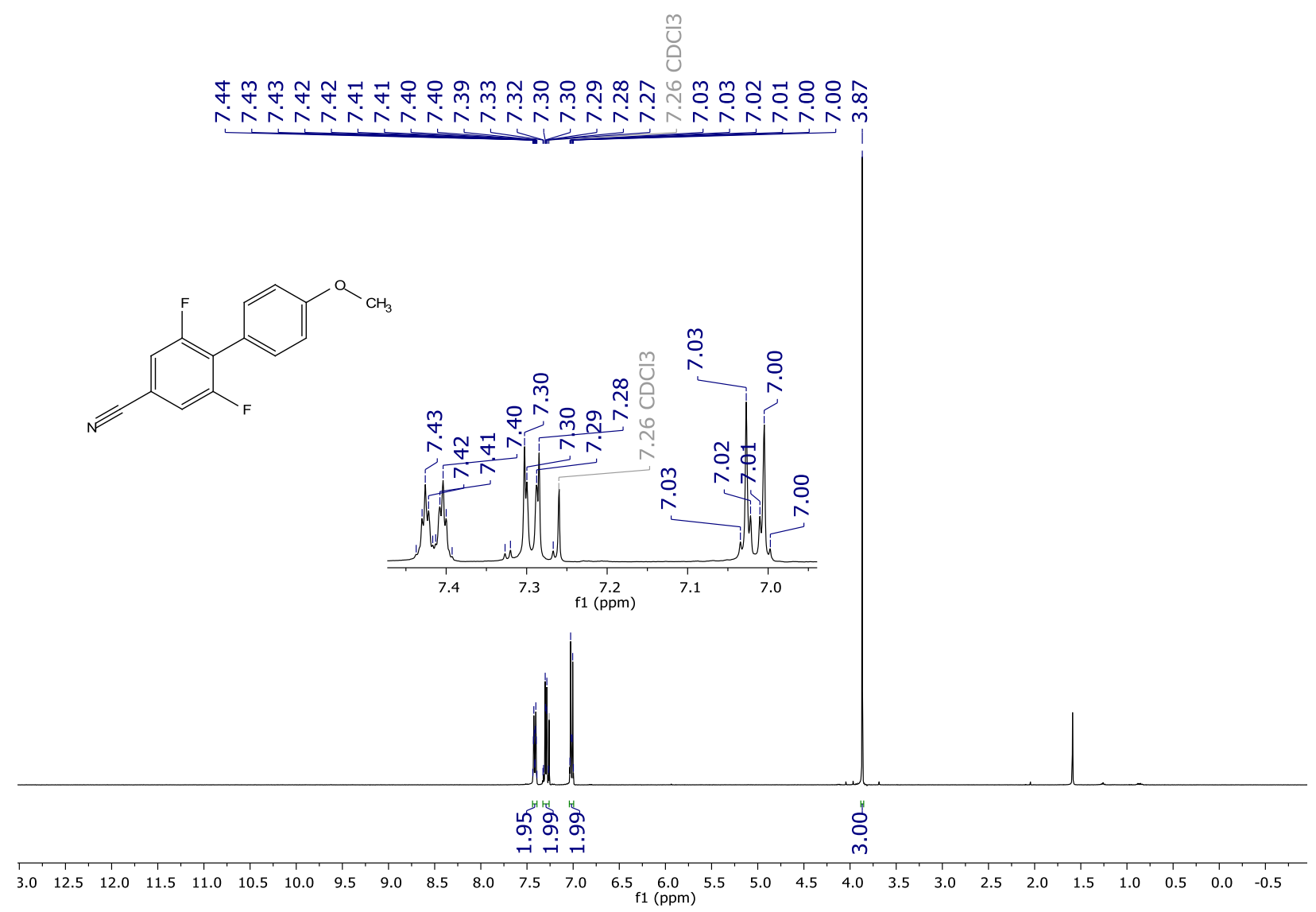

${ }^{1} \mathrm{H}$ NMR $\left(400 \mathrm{MHz}, \mathrm{CDCl}_{3}\right.$ ) spectrum of $\mathbf{7 i}$.

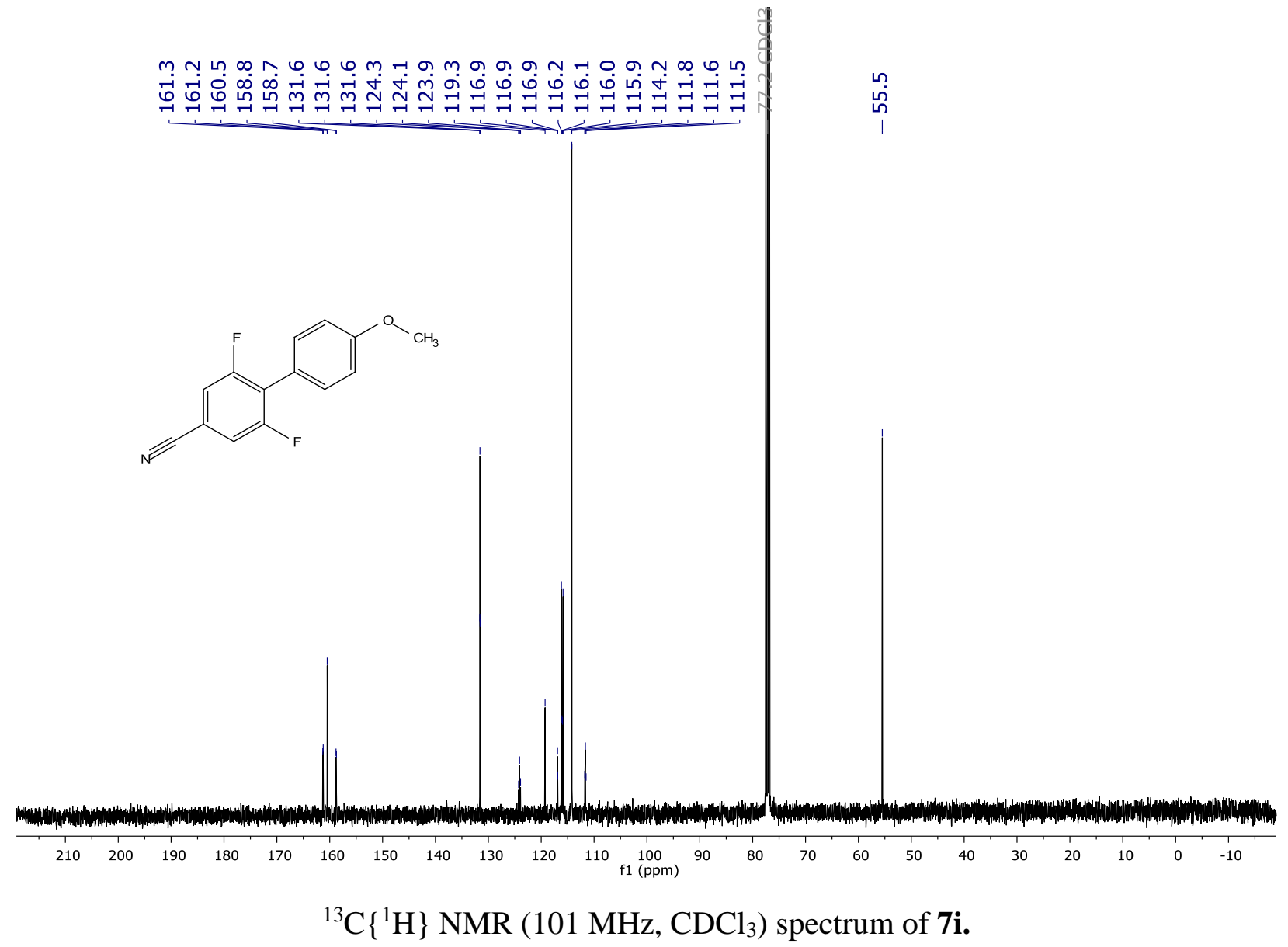



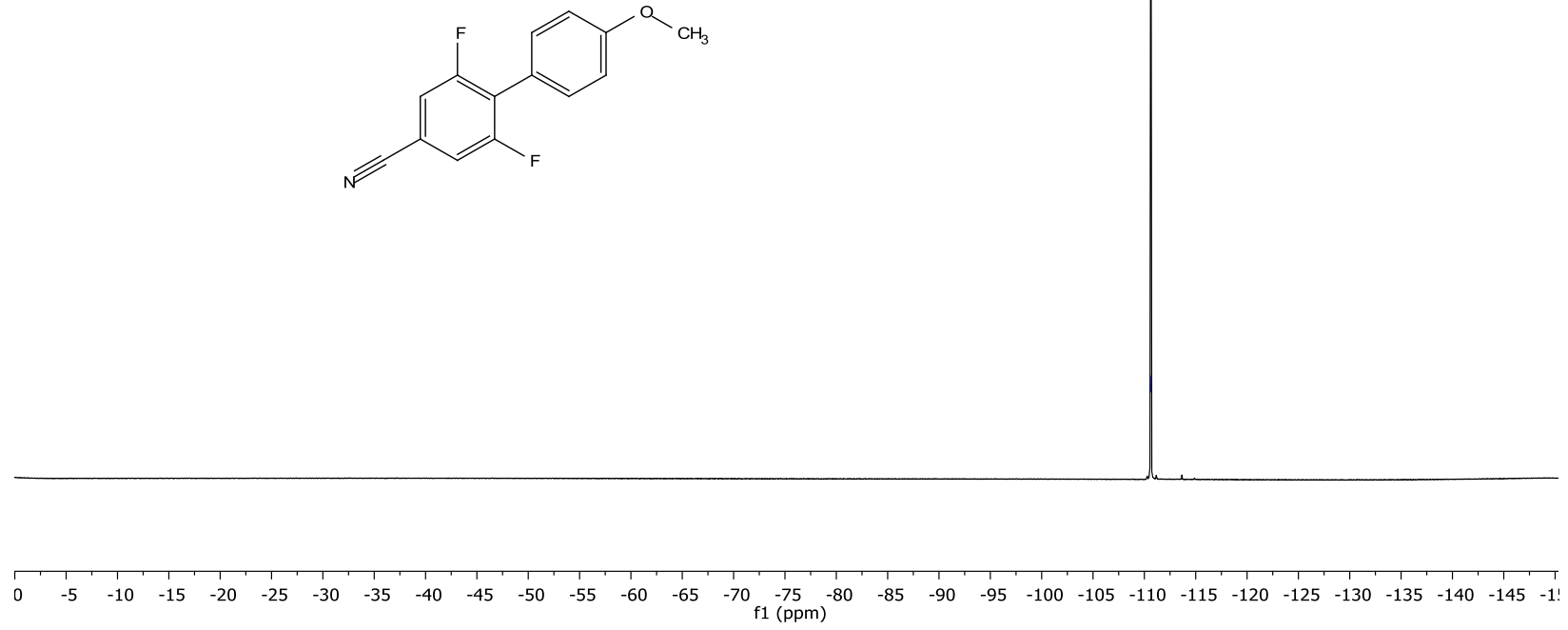

${ }^{19} \mathrm{~F}$ NMR (282 MHz, $\left.\mathrm{CDCl}_{3}\right)$ spectrum of $7 \mathbf{i}$.

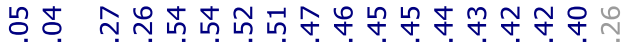

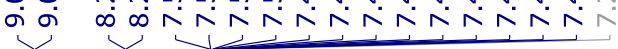

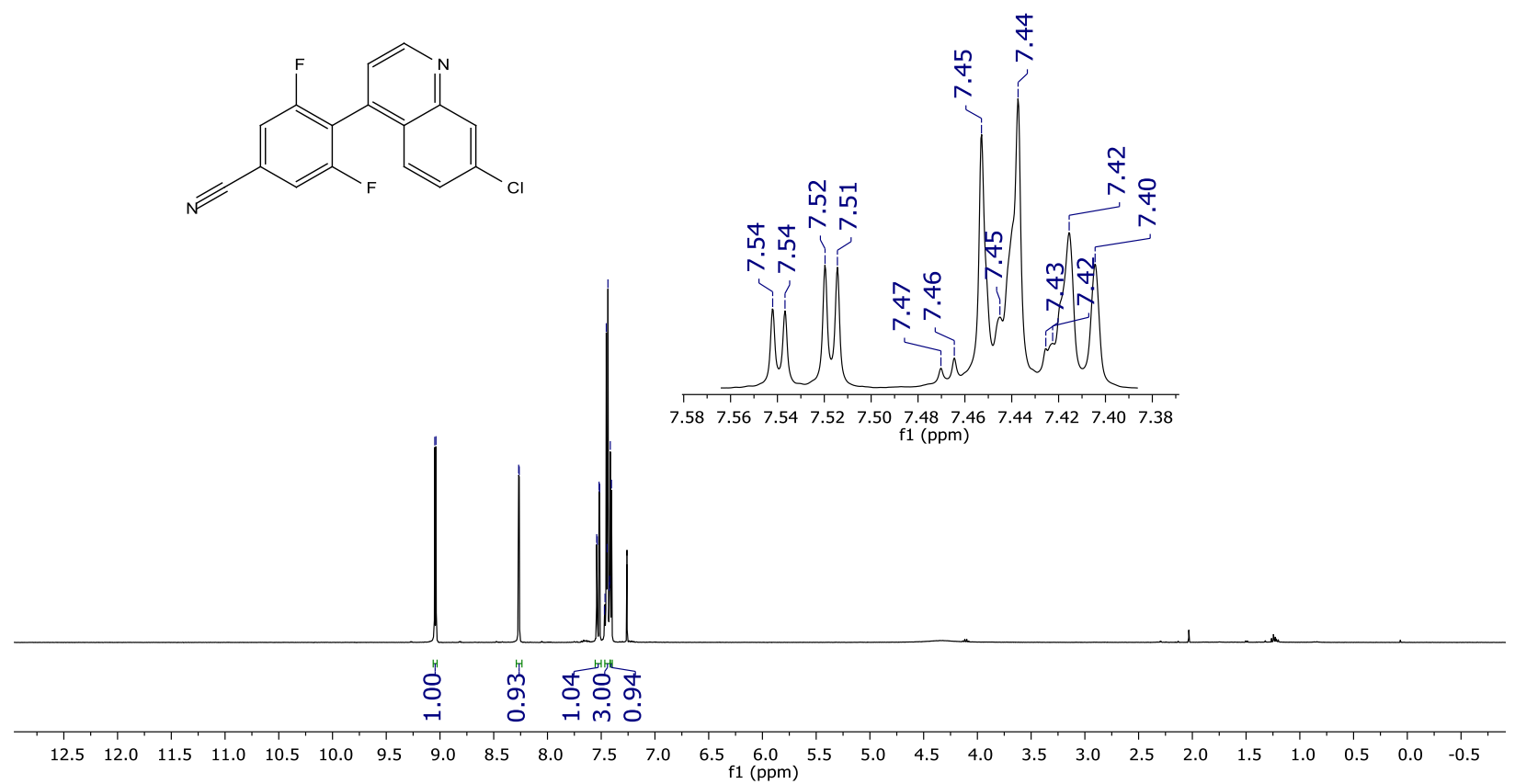

${ }^{1} \mathrm{H}$ NMR (400 MHz, $\mathrm{CDCl}_{3}$ ) spectrum of $\mathbf{7 j}$. 


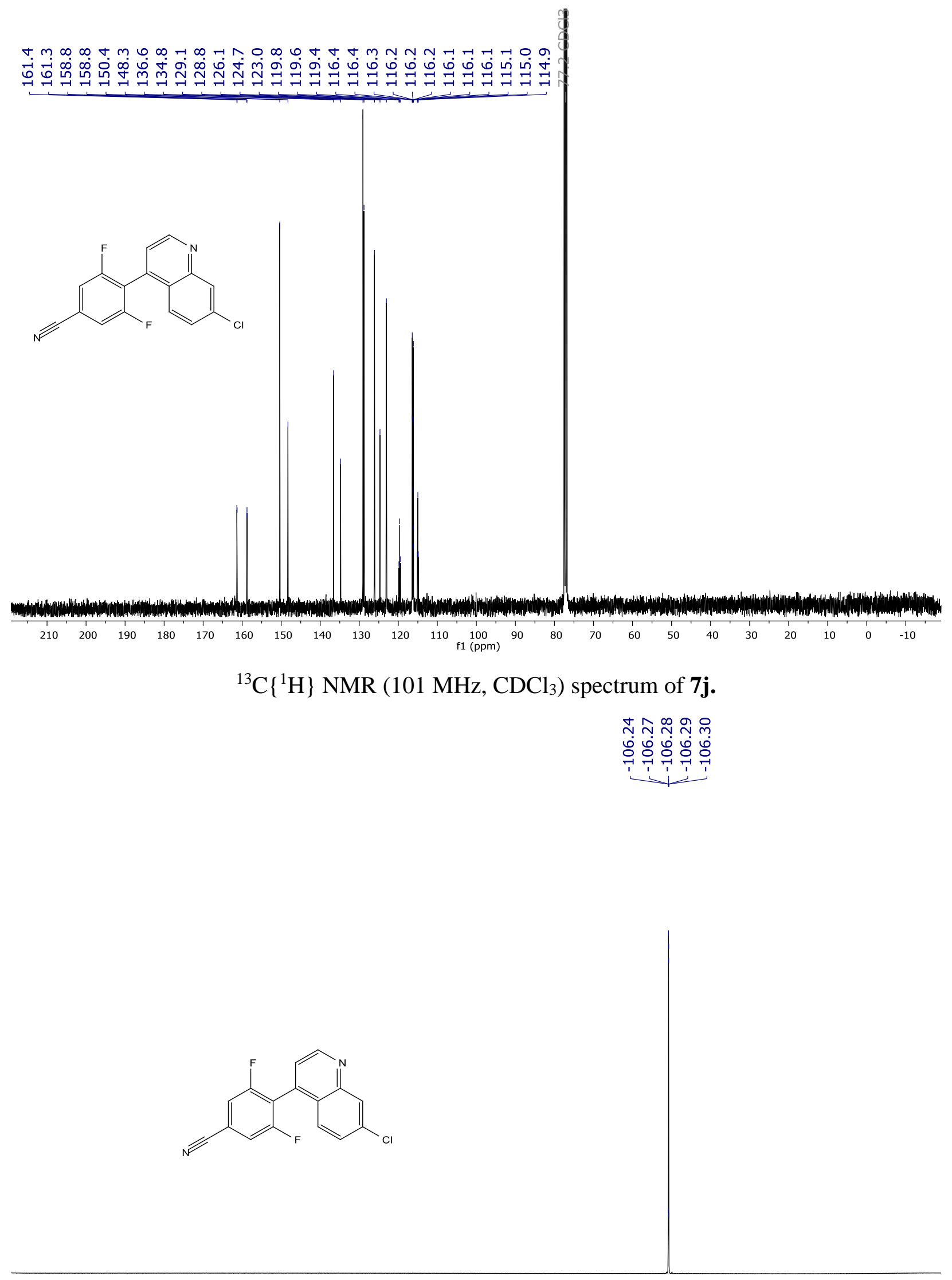

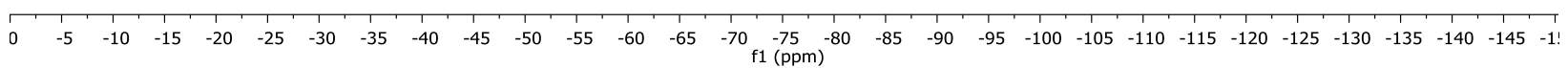

${ }^{19} \mathrm{~F}$ NMR (282 MHz, $\left.\mathrm{CDCl}_{3}\right)$ spectrum of $\mathbf{7 j}$. 

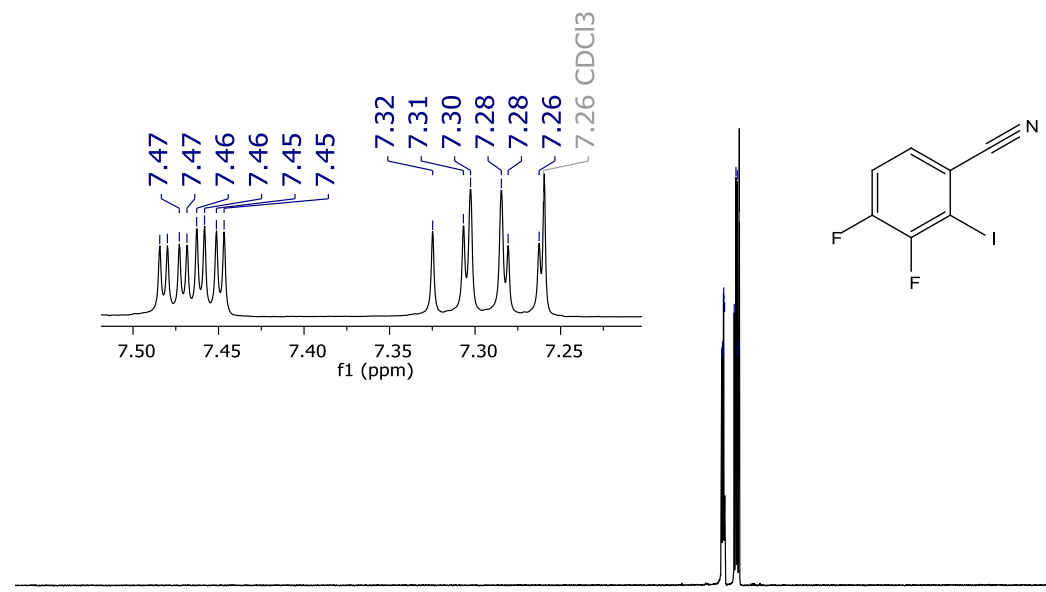

엄

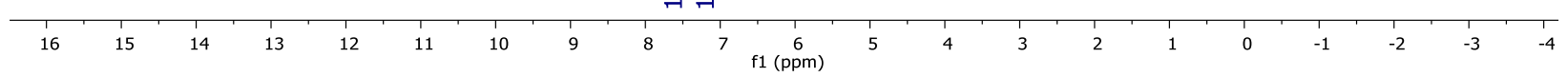

${ }^{1} \mathrm{H}$ NMR $\left(400 \mathrm{MHz}, \mathrm{CDCl}_{3}\right.$ ) spectrum of $\mathbf{7 k}$.

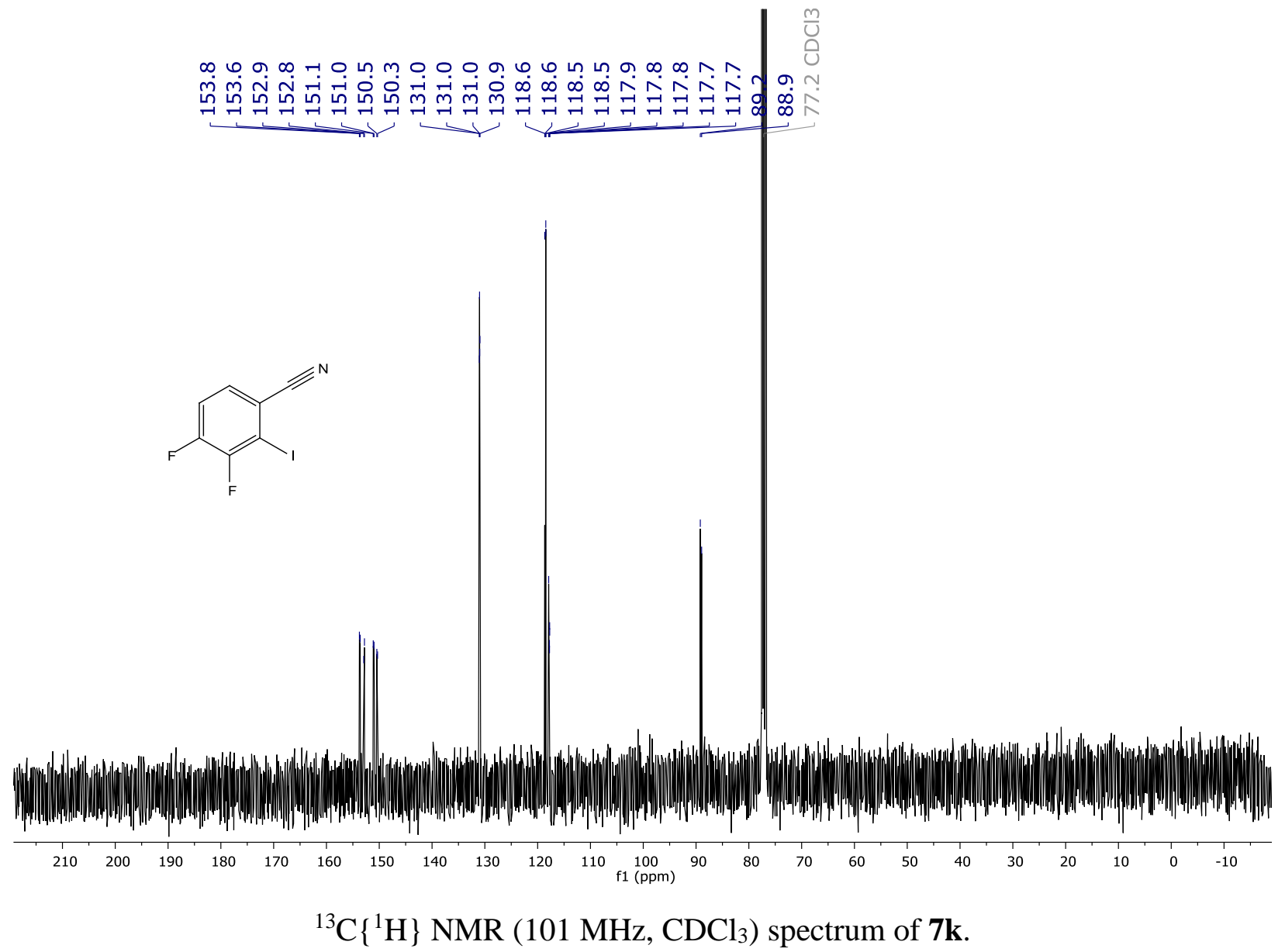



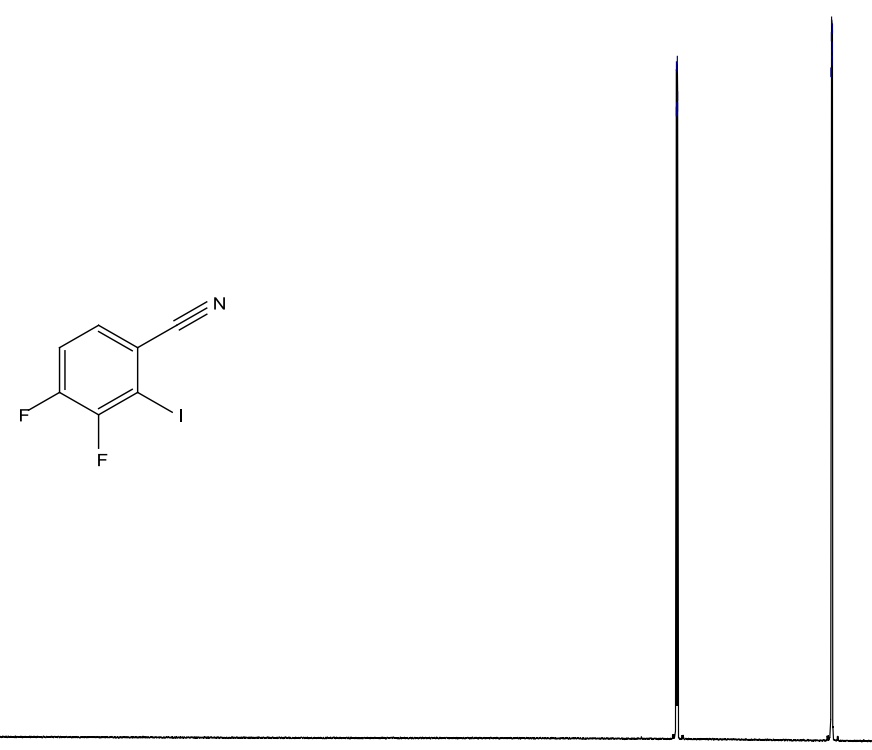

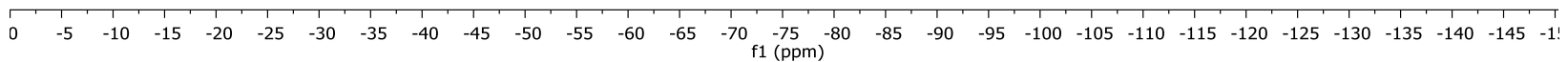

${ }^{19} \mathrm{~F}$ NMR $\left(282 \mathrm{MHz}, \mathrm{CDCl}_{3}\right)$ spectrum of $\mathbf{7 k}$.

员

只

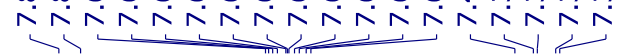
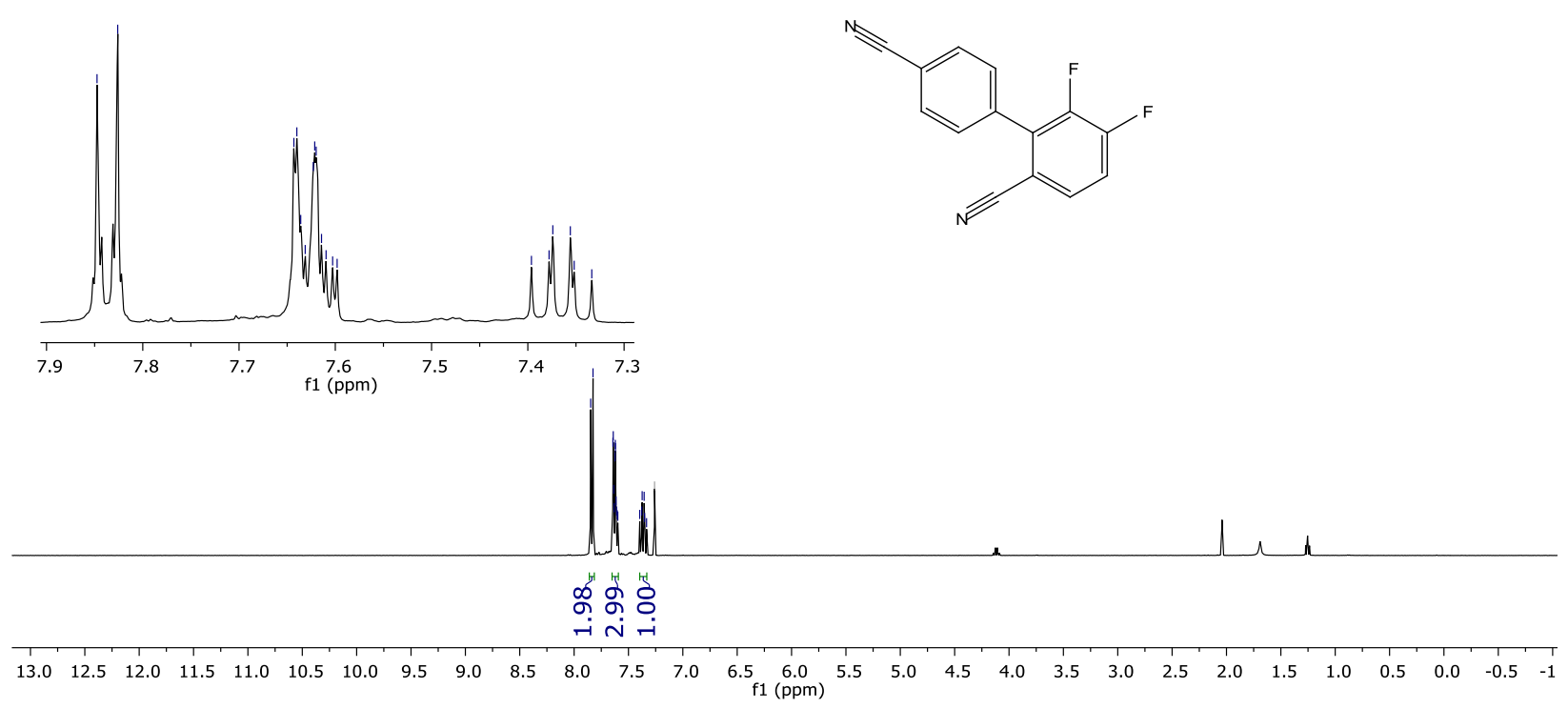

${ }^{1} \mathrm{H}$ NMR (400 MHz, $\mathrm{CDCl}_{3}$ ) spectrum of 71. 


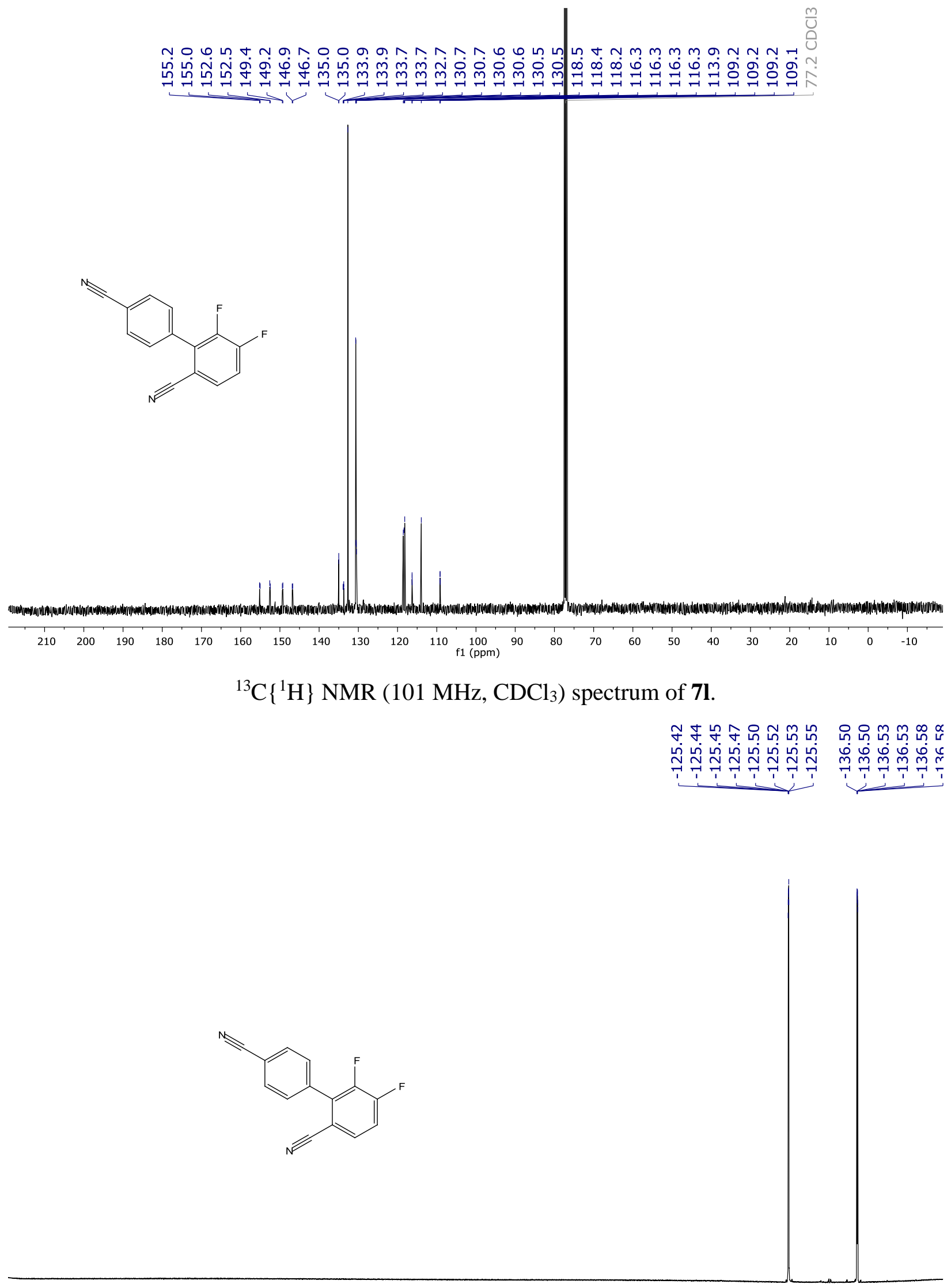

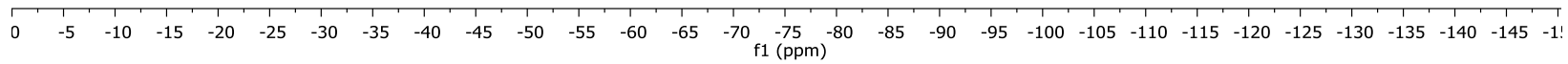

${ }^{19} \mathrm{~F}$ NMR (282 MHz, $\left.\mathrm{CDCl}_{3}\right)$ spectrum of 71. 


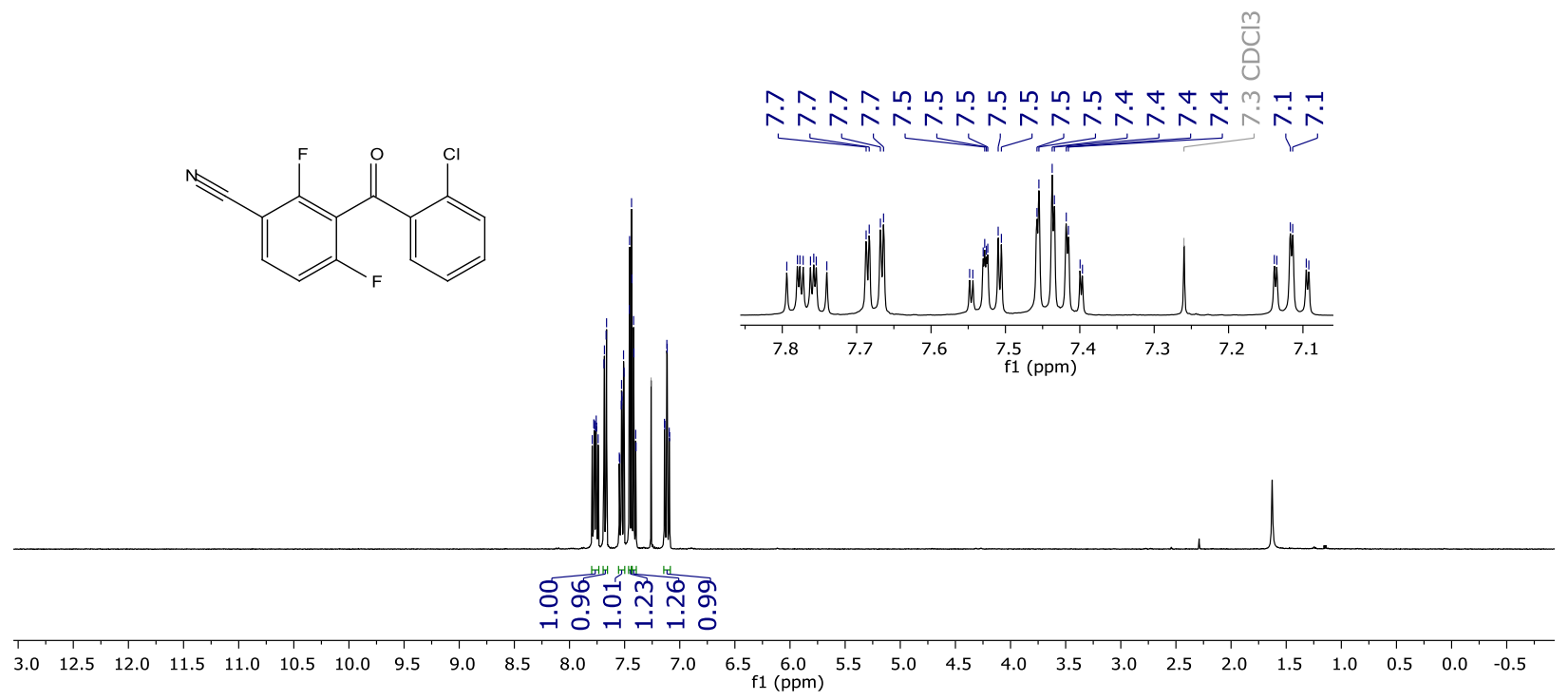

${ }^{1} \mathrm{H}$ NMR (400 MHz, $\mathrm{CDCl}_{3}$ ) spectrum of $\mathbf{7 m}$.

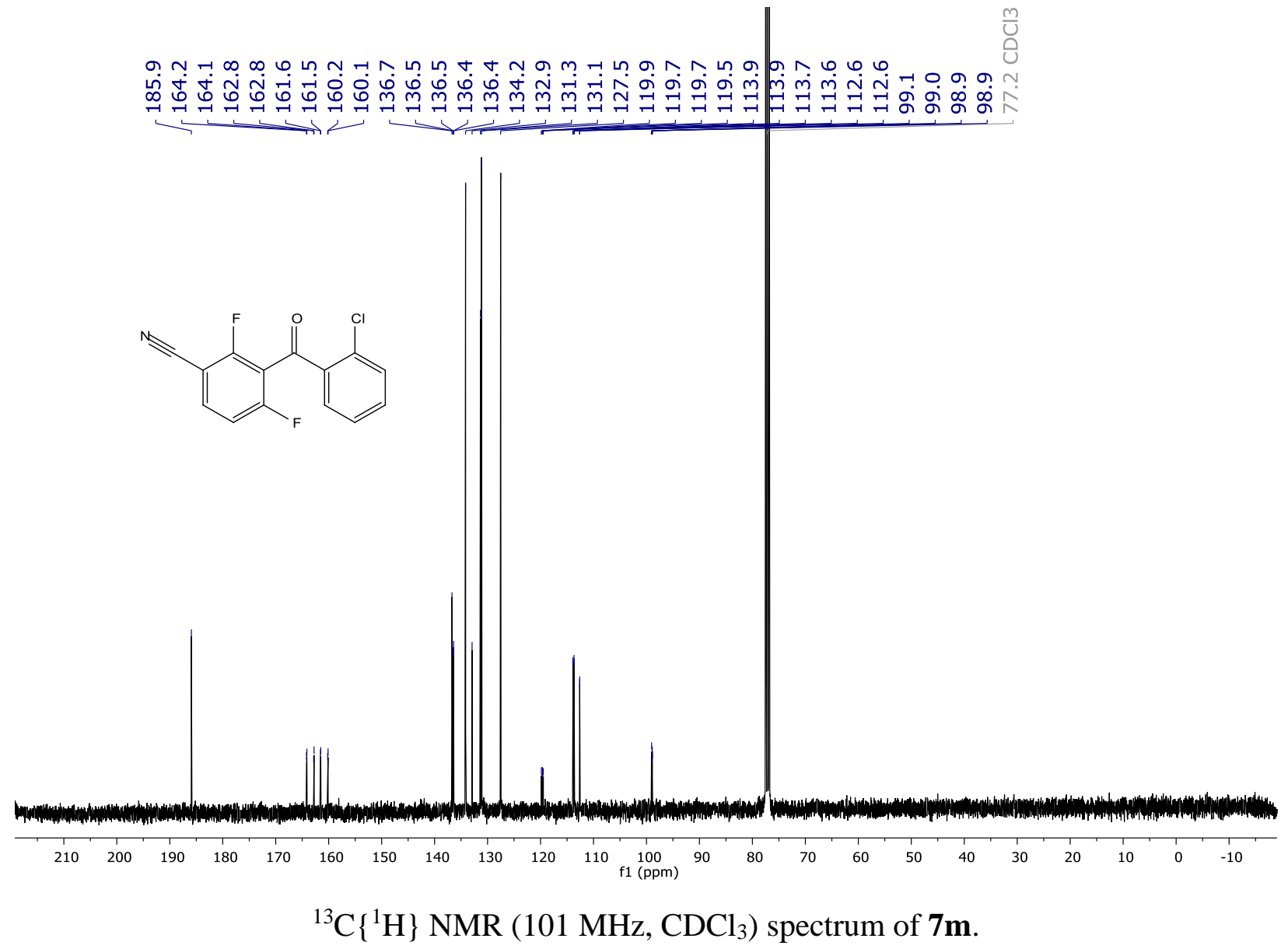




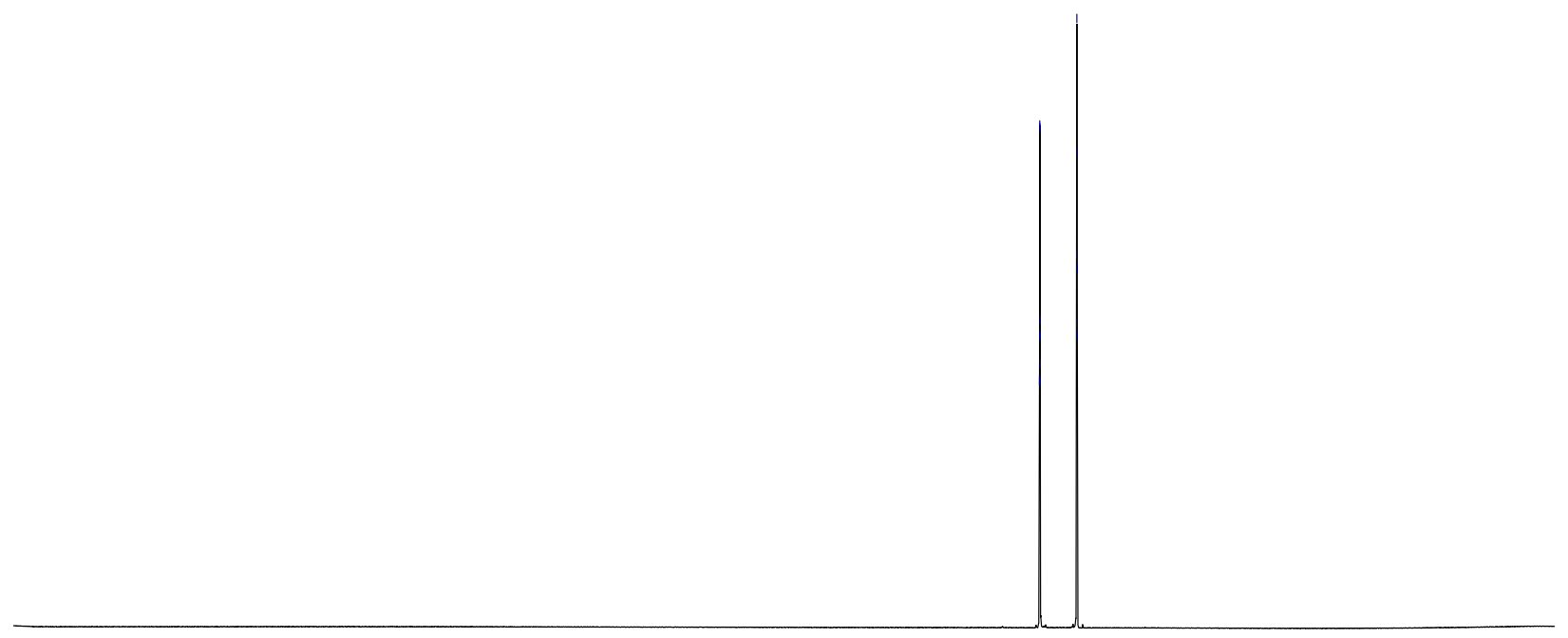

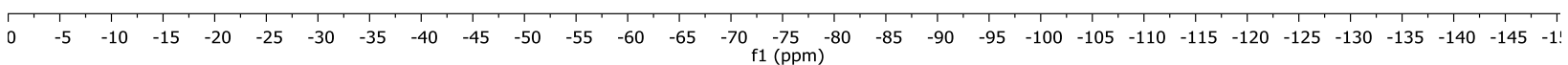

${ }^{19} \mathrm{~F}$ NMR $\left(282 \mathrm{MHz}, \mathrm{CDCl}_{3}\right)$ spectrum of $\mathbf{7 m}$.

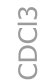

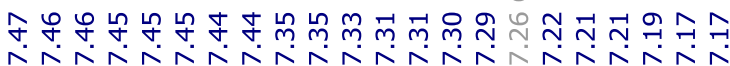

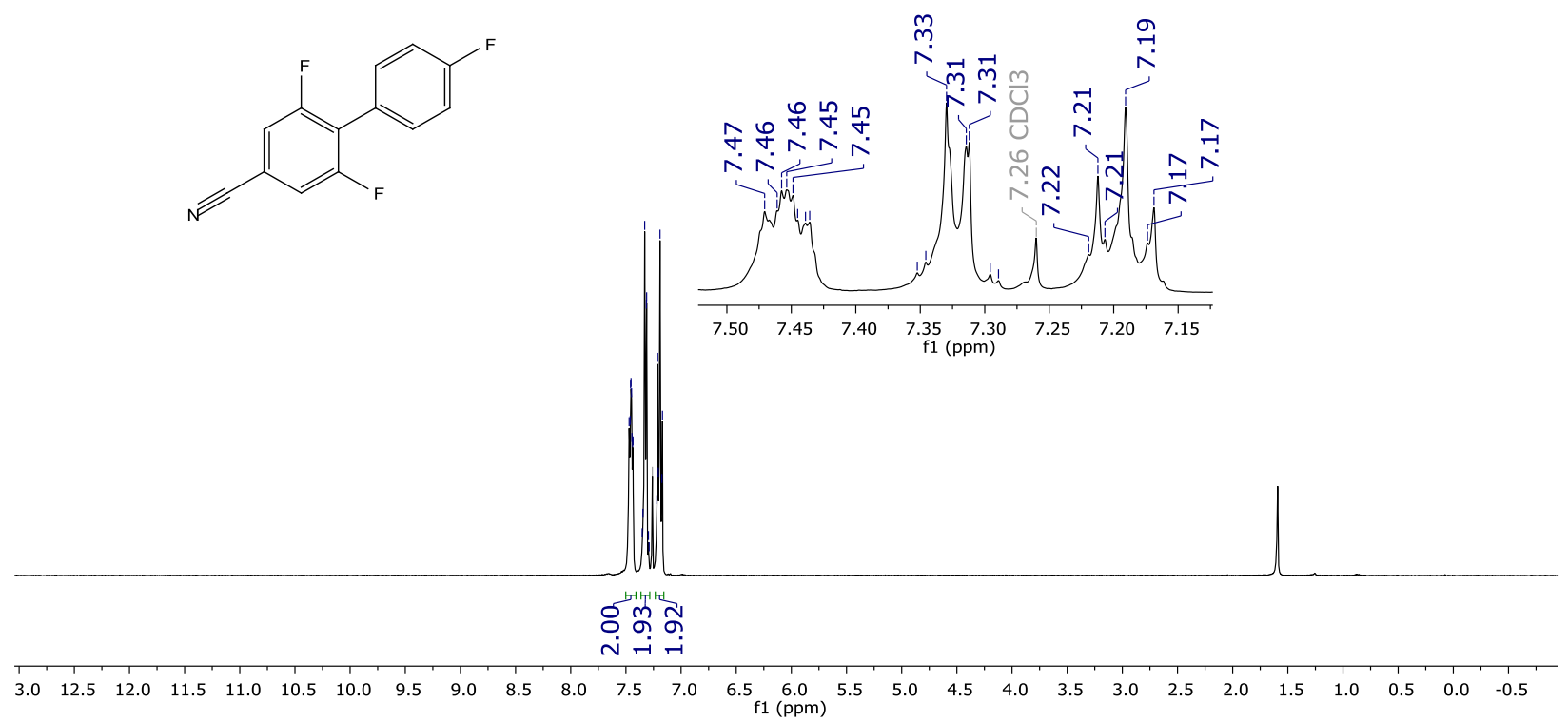

${ }^{1} \mathrm{H}$ NMR (400 MHz, $\mathrm{CDCl}_{3}$ ) spectrum of $\mathbf{7 n}$. 


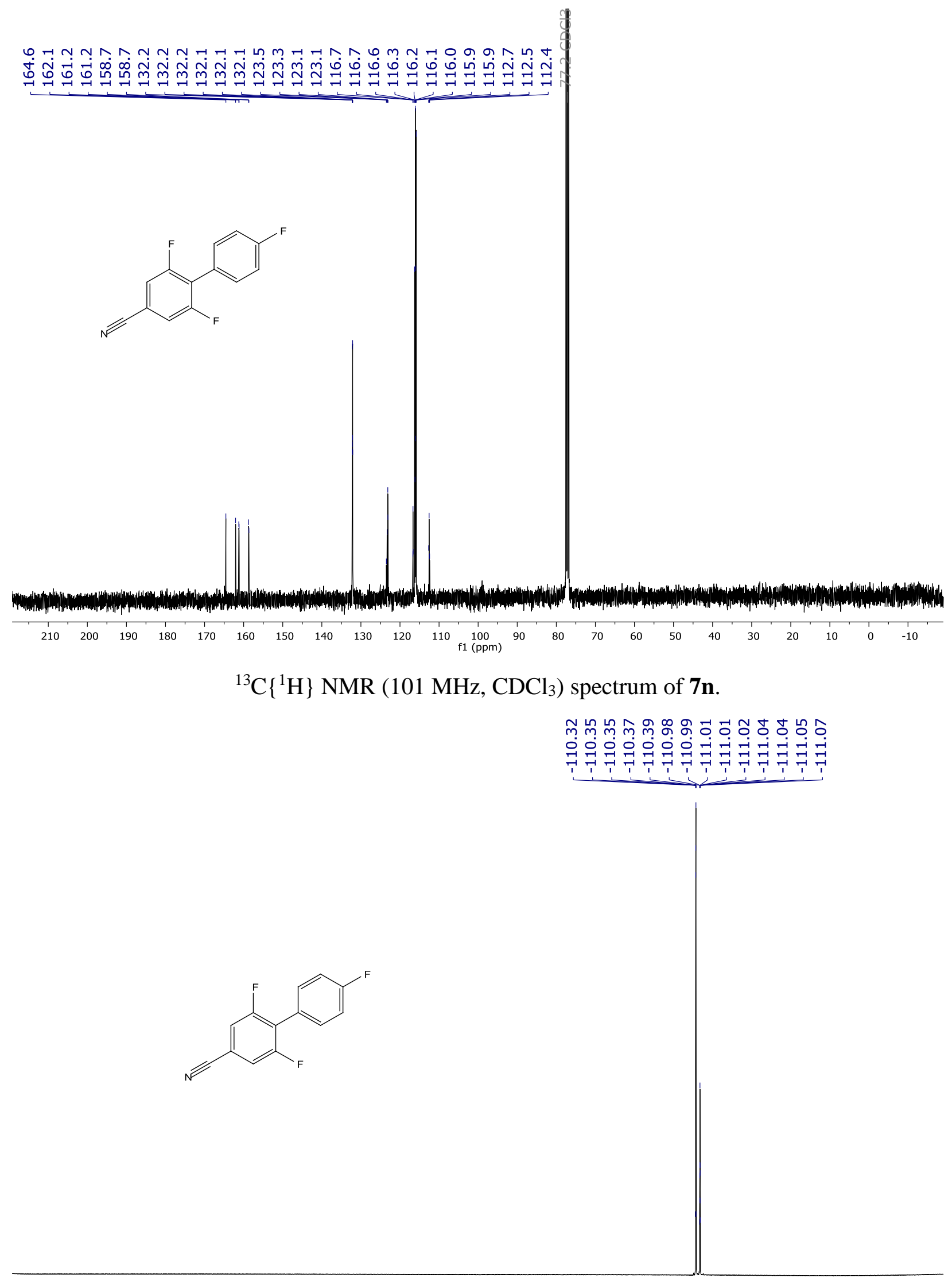

\begin{tabular}{llllllllllllllllllllllllllllllllllllllllllllllllllllllll}
\hline 0 & -5 & -10 & -15 & -20 & -25 & -30 & -35 & -40 & -45 & -50 & -55 & -60 & -65 & -70 & -75 & -80 & -85 & -90 & -95 & -100 & -105 & -110 & -115 & -120 & -125 & -130 & -135 & -140 & -145 & -1
\end{tabular}

${ }^{19} \mathrm{~F}$ NMR (282 MHz, $\mathrm{CDCl}_{3}$ ) spectrum of $\mathbf{7 n}$. 


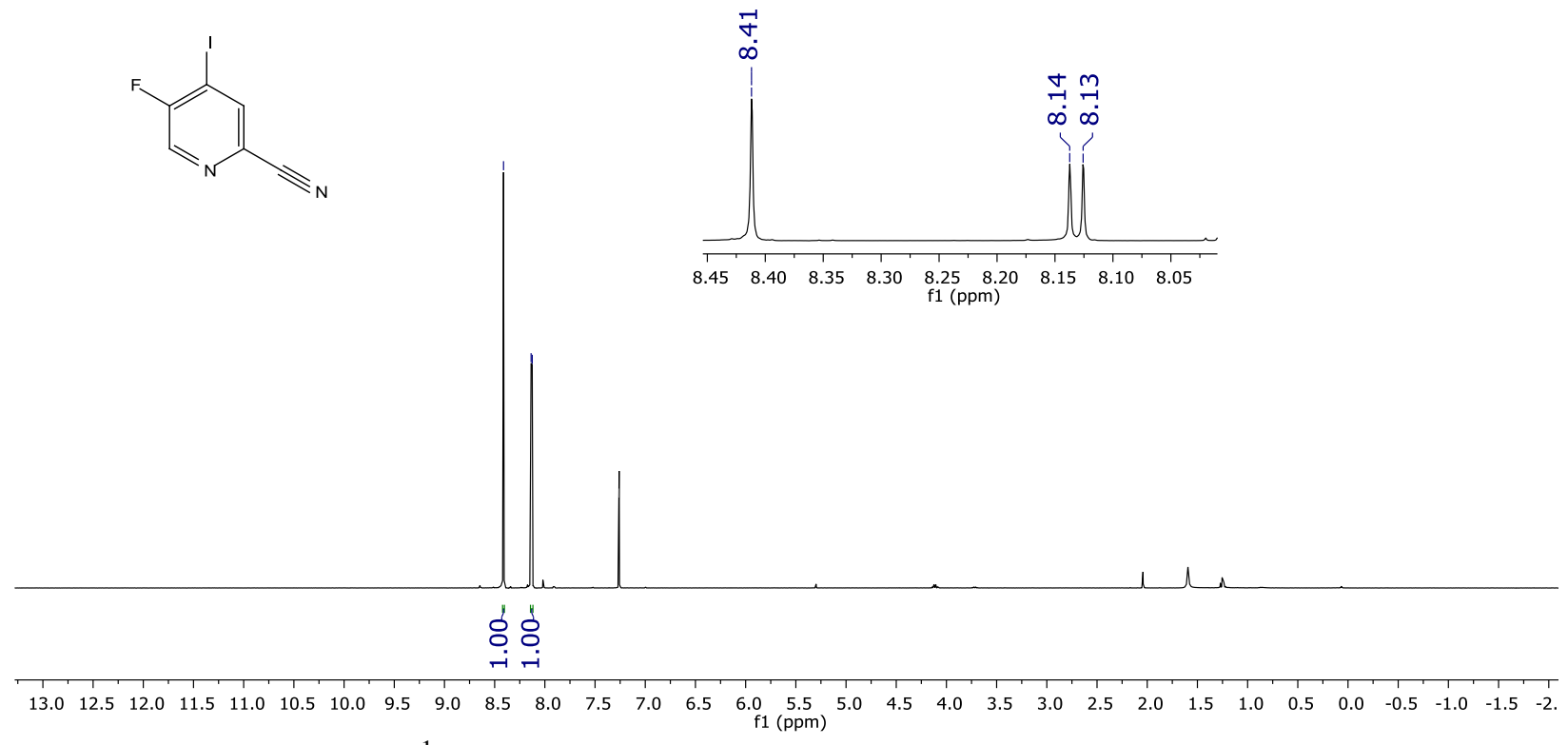

${ }^{1} \mathrm{H}$ NMR (400 MHz, $\mathrm{CDCl}_{3}$ ) spectrum of $\mathbf{1 0 a}$.

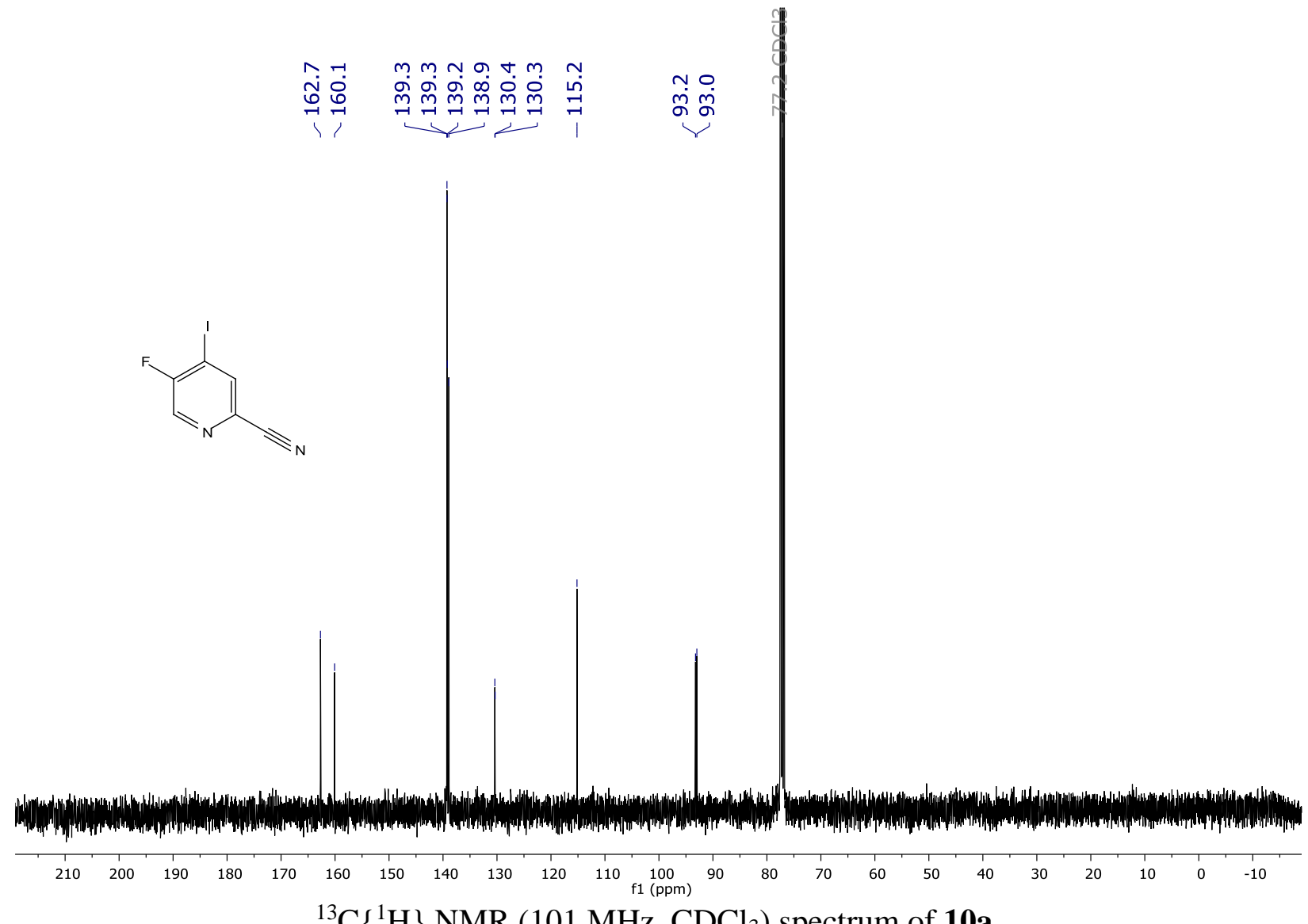

${ }^{13} \mathrm{C}\left\{{ }^{1} \mathrm{H}\right\}$ NMR (101 MHz, $\left.\mathrm{CDCl}_{3}\right)$ spectrum of $\mathbf{1 0 a}$. 


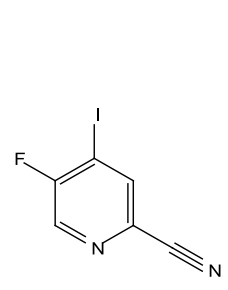

\begin{tabular}{llllllllllllllllllllllllllllllllll}
\hline & -5 & -10 & -15 & -20 & -25 & -30 & -35 & -40 & -45 & -50 & -55 & -60 & -65 & -70 & -75 & -80 & -85 & -90 & -95 & -100 & -105 & -110 & -115 & -120 & -125 & -130 & -135 & -140 & -145 & -1
\end{tabular} ${ }^{19} \mathrm{~F}$ NMR $\left(282 \mathrm{MHz}, \mathrm{CDCl}_{3}\right)$ spectrum of $\mathbf{1 0 a}$.
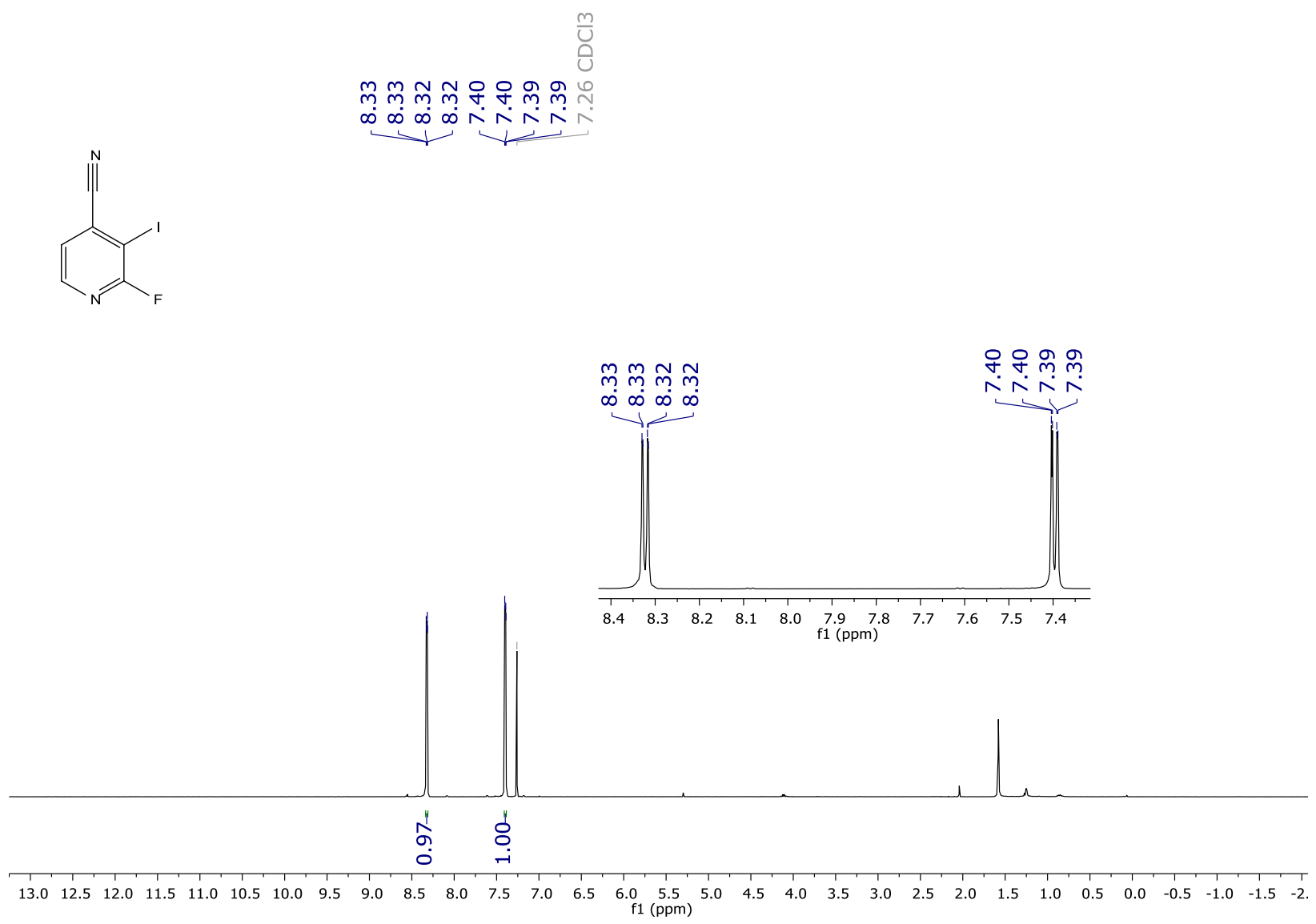

${ }^{1} \mathrm{H}$ NMR (400 MHz, $\mathrm{CDCl}_{3}$ ) spectrum of $\mathbf{1 0 b}$. 


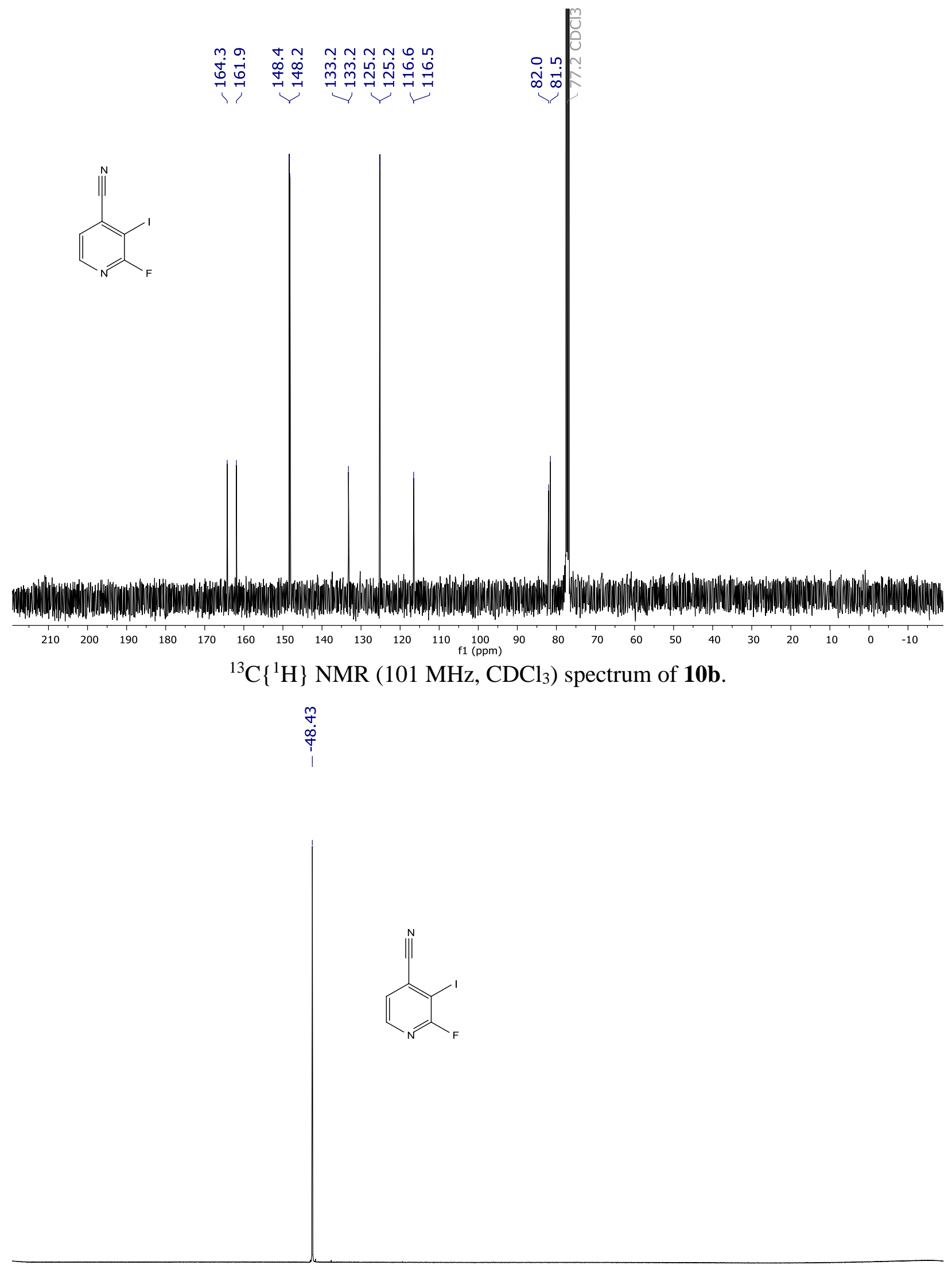

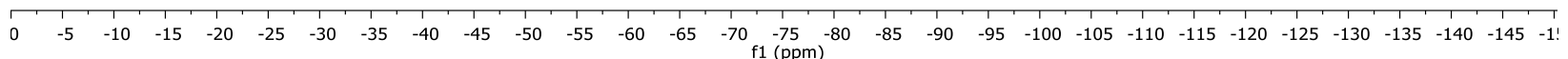

${ }^{19} \mathrm{~F}$ NMR $\left(282 \mathrm{MHz}, \mathrm{CDCl}_{3}\right)$ spectrum of $\mathbf{1 0 b}$. 


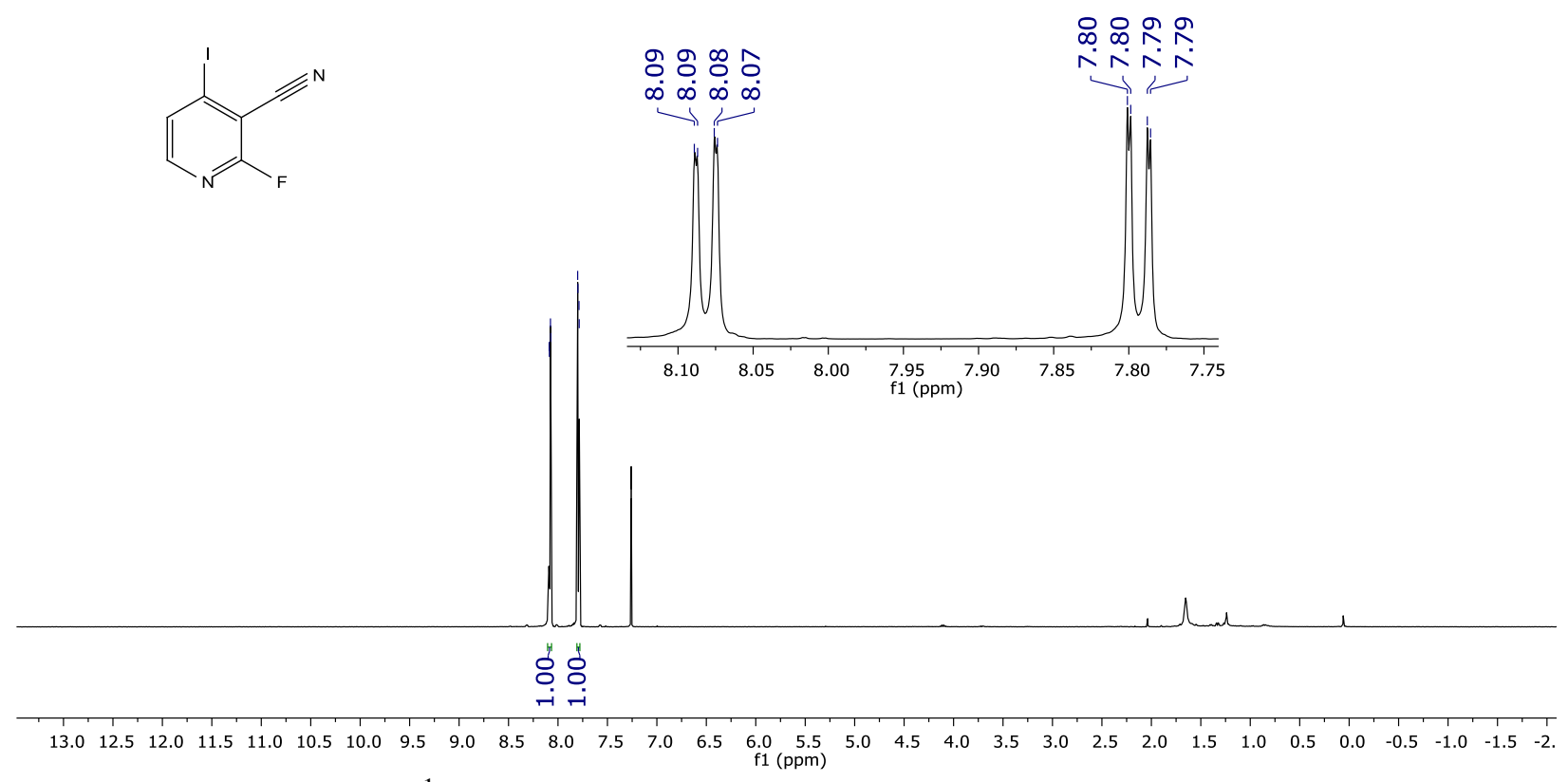

${ }^{1} \mathrm{H} \mathrm{NMR}\left(400 \mathrm{MHz}, \mathrm{CDCl}_{3}\right.$ ) spectrum of 10c.

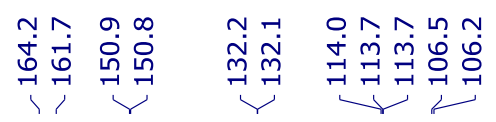
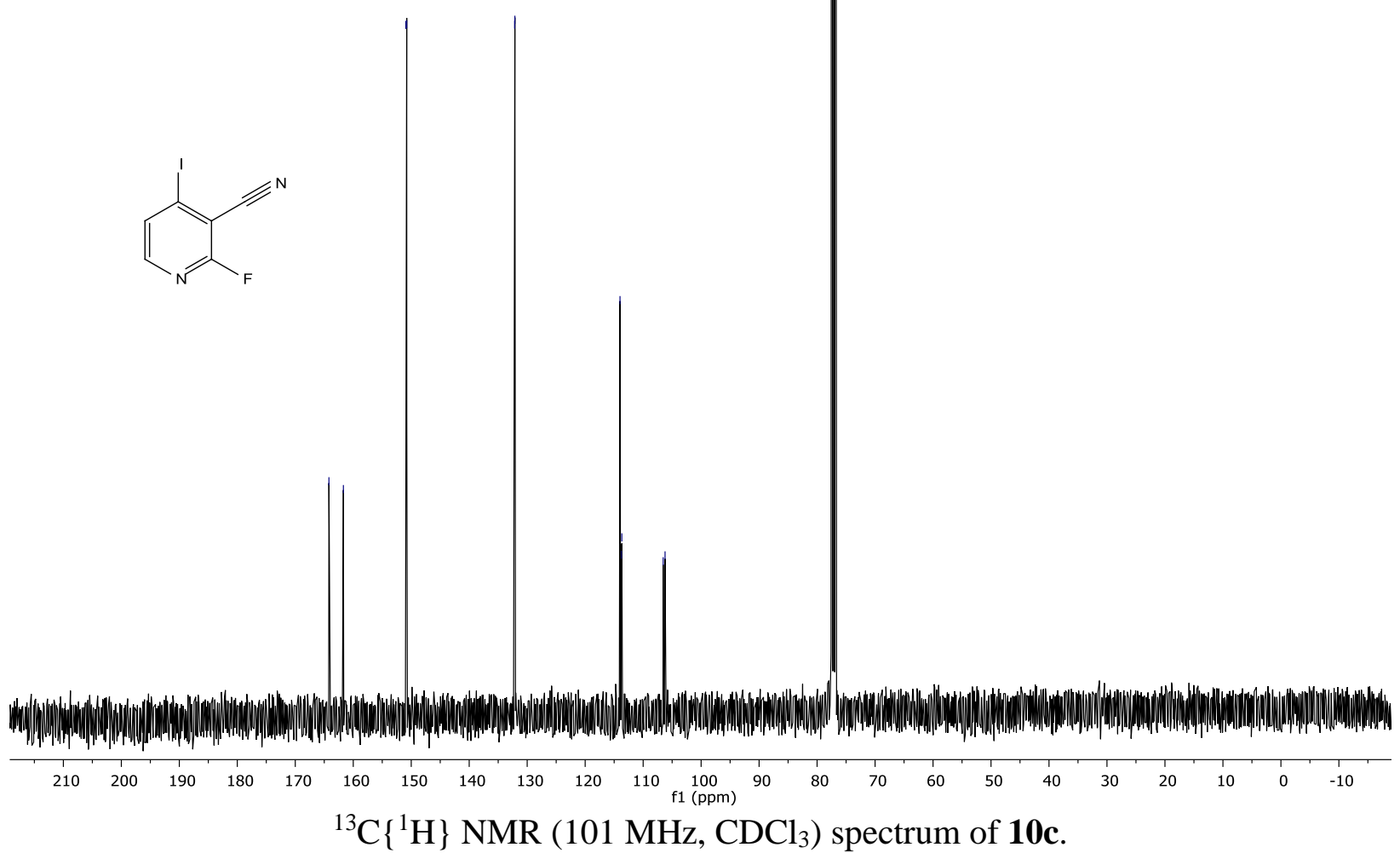

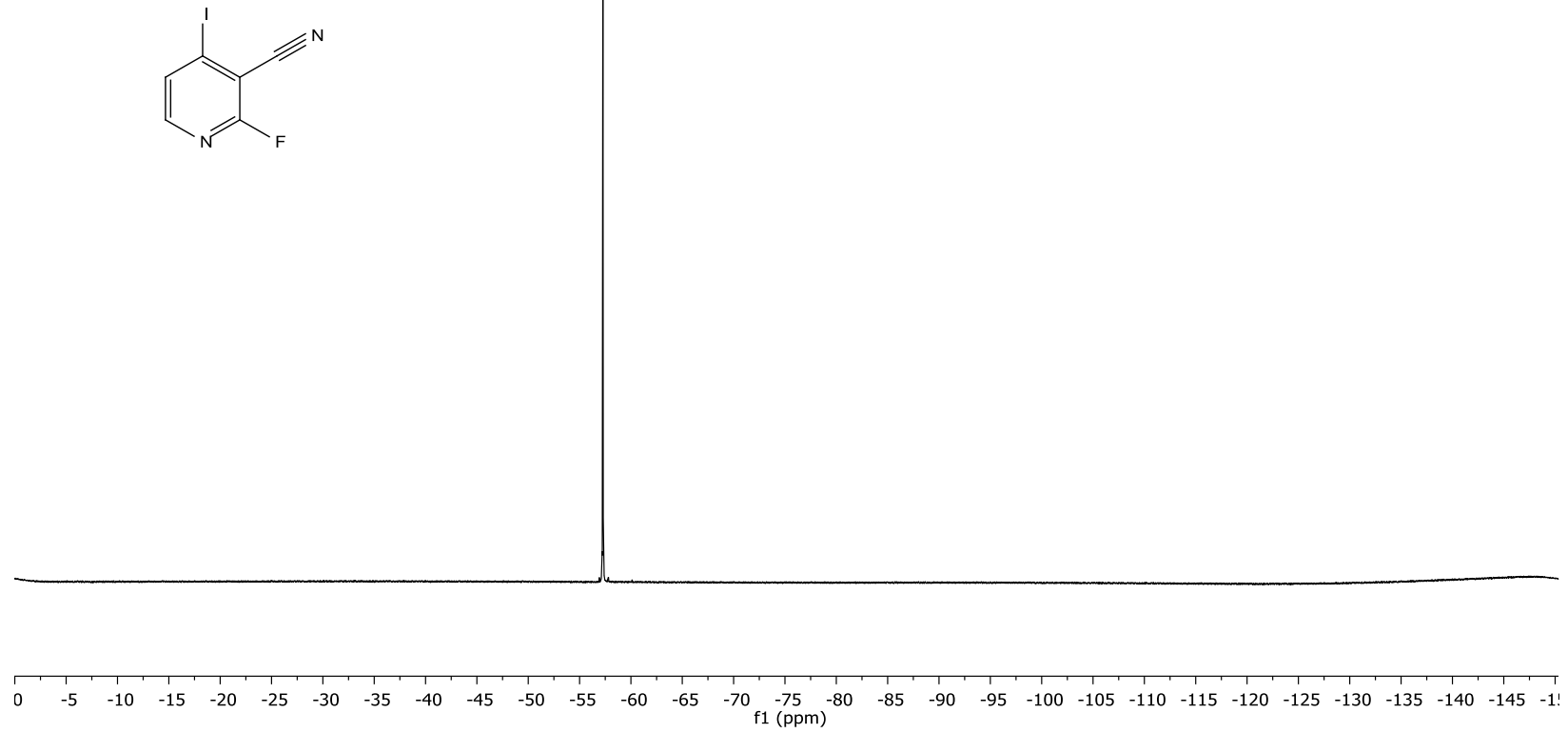

${ }^{19} \mathrm{~F}$ NMR $\left(282 \mathrm{MHz}, \mathrm{CDCl}_{3}\right)$ spectrum of $\mathbf{1 0 c}$.

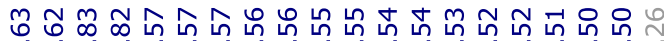

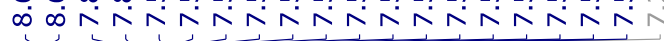

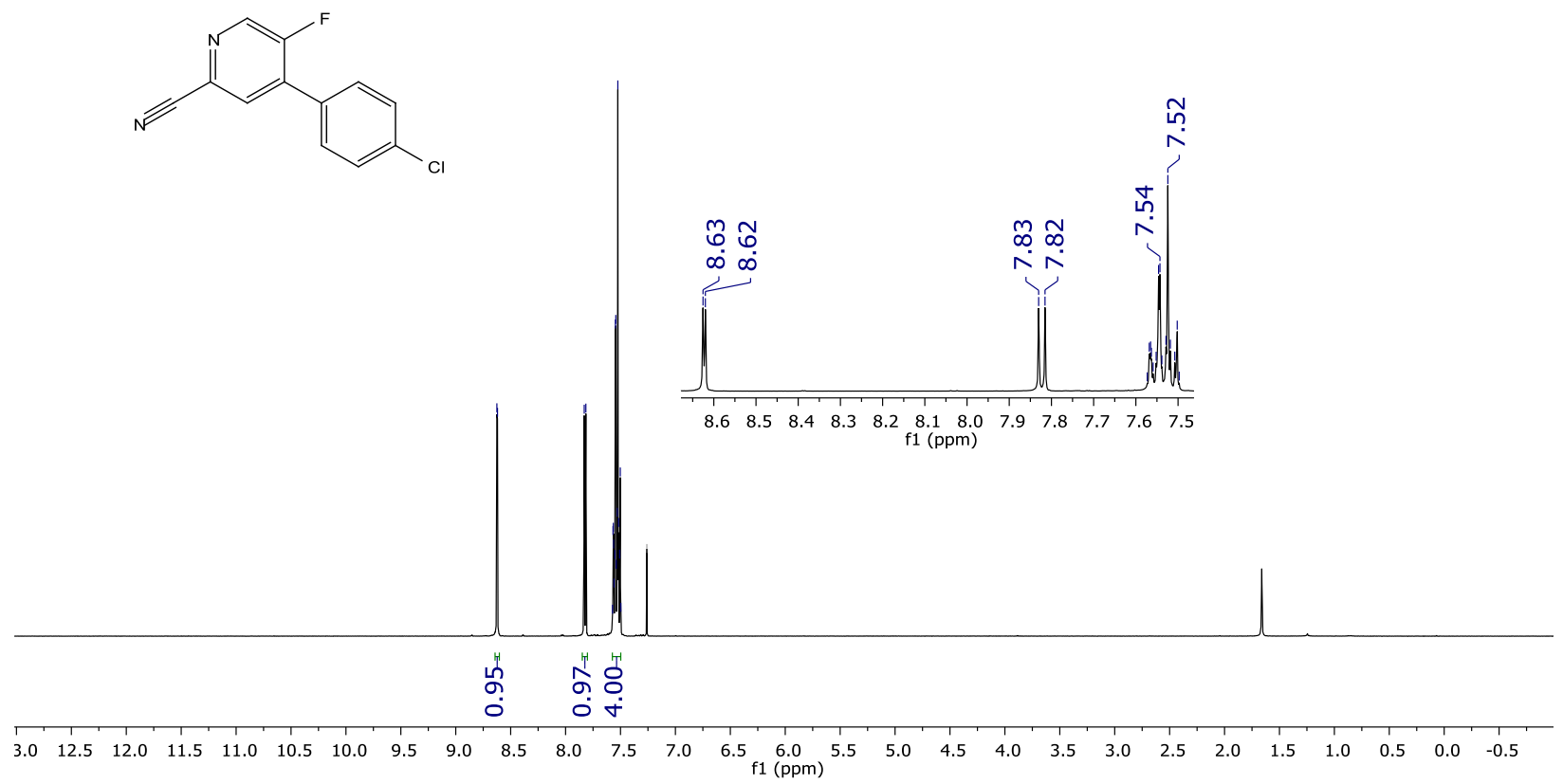

${ }^{1} \mathrm{H}$ NMR $\left(400 \mathrm{MHz}, \mathrm{CDCl}_{3}\right.$ ) spectrum of $\mathbf{1 0 d}$. 


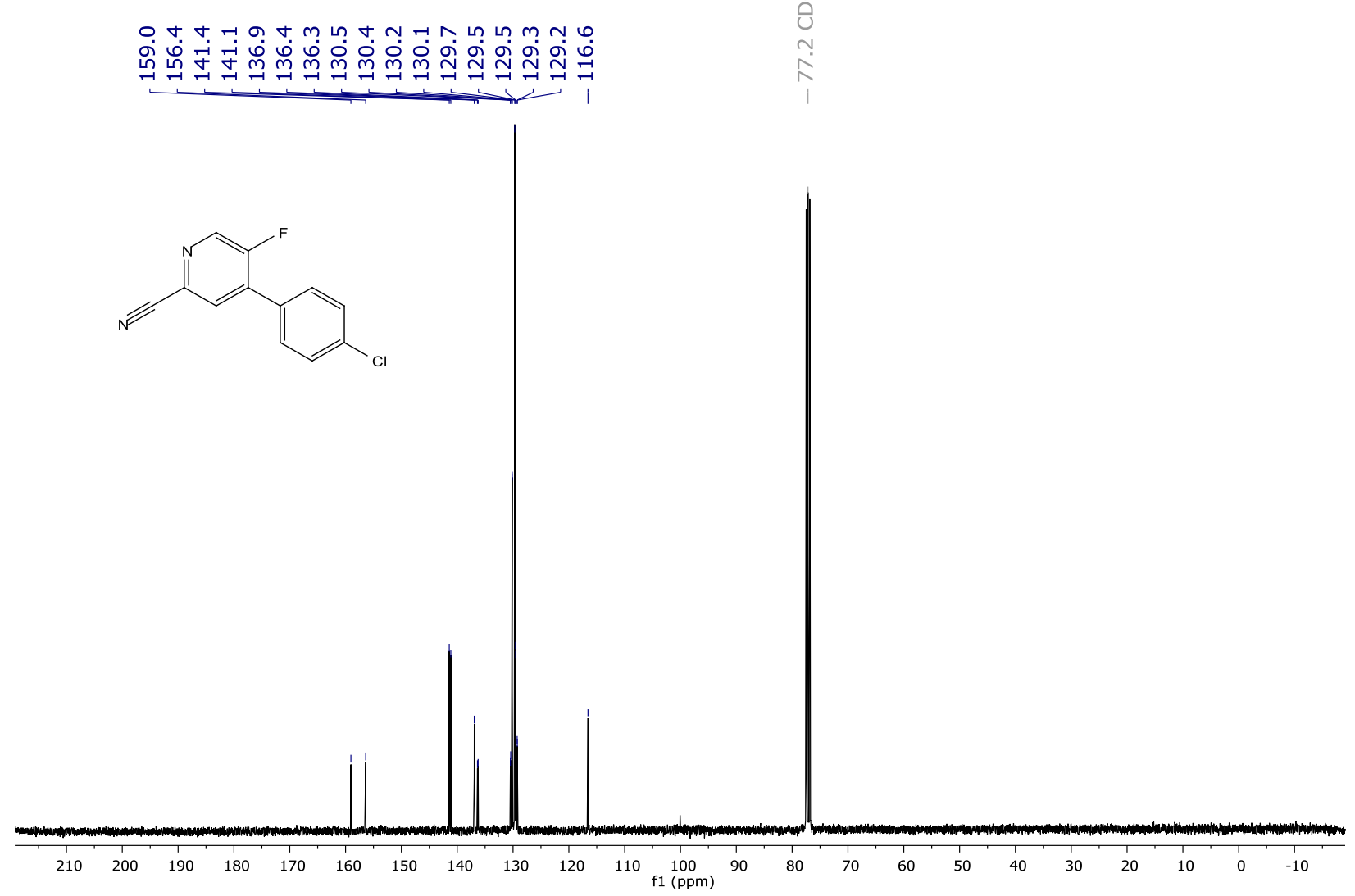

${ }^{13} \mathrm{C}\left\{{ }^{1} \mathrm{H}\right\}$ NMR $\left(101 \mathrm{MHz}, \mathrm{CDCl}_{3}\right)$ spectrum of $\mathbf{1 0 d}$.

守守守守守守守

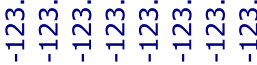
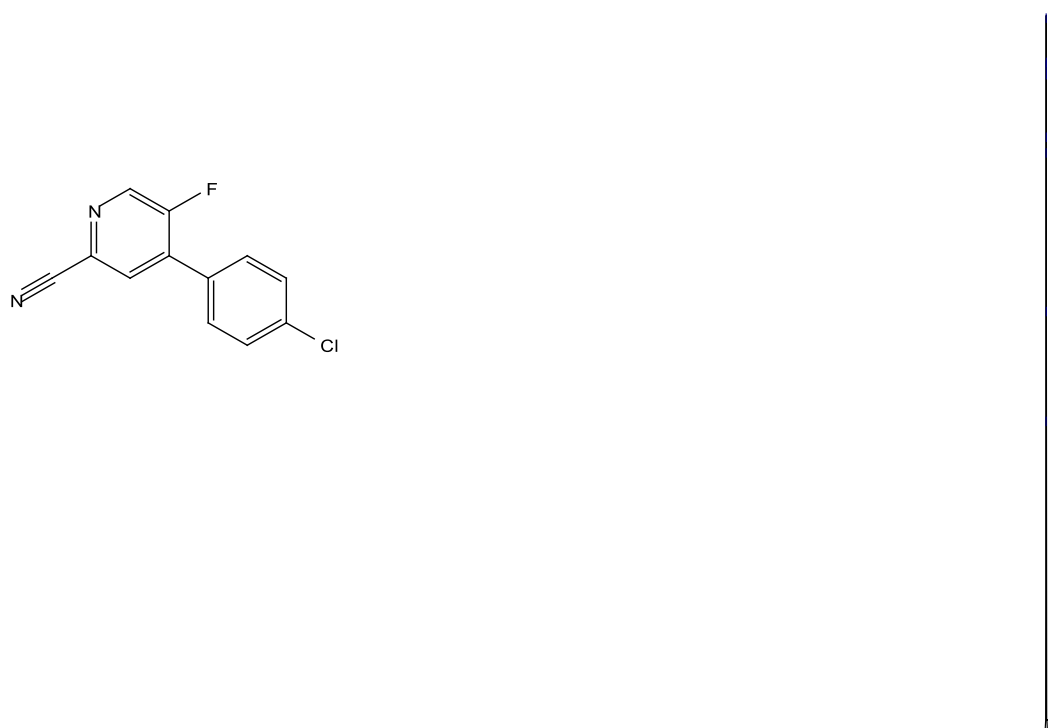

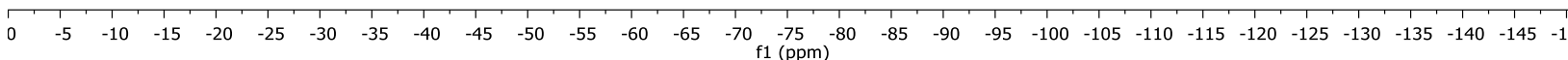

${ }^{19} \mathrm{~F}$ NMR $\left(282 \mathrm{MHz}, \mathrm{CDCl}_{3}\right)$ spectrum of $\mathbf{1 0 d}$. 
<smiles>N#Cc1cc(C(O)c2ccc(Br)s2)c(F)cn1</smiles>

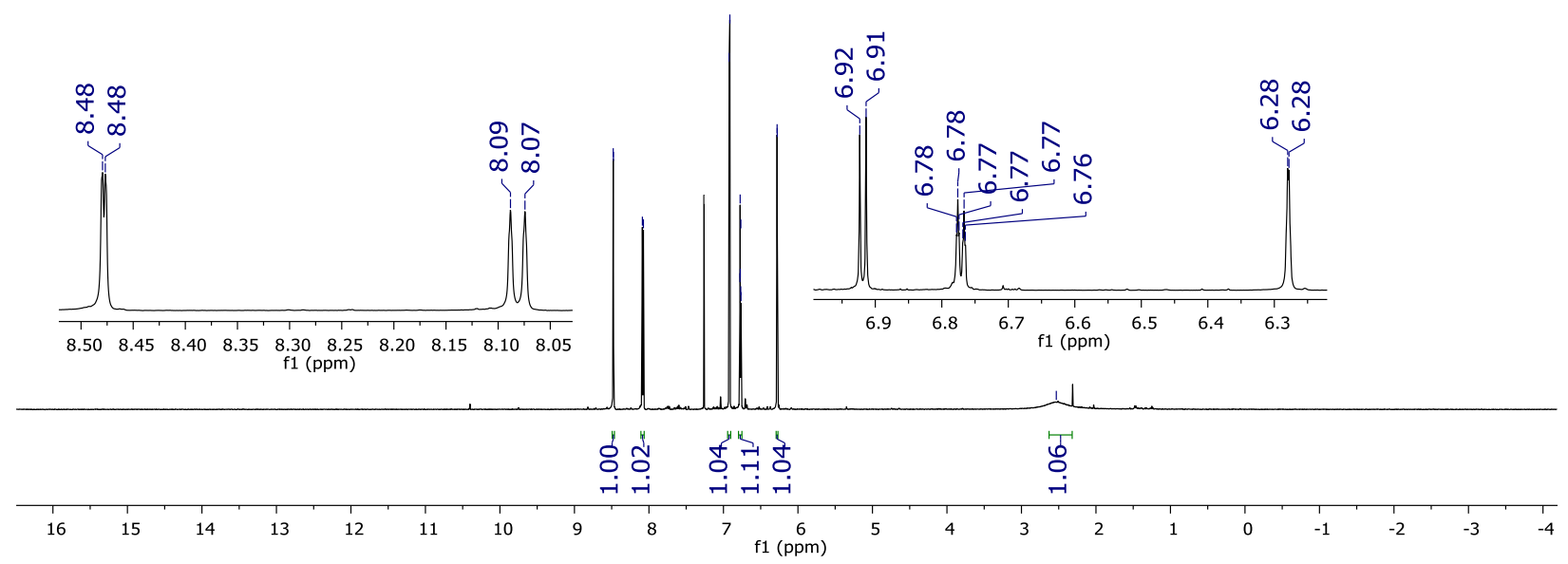

${ }^{1} \mathrm{H}$ NMR (400 MHz, $\mathrm{CDCl}_{3}$ ) spectrum of $\mathbf{1 0 e}$.

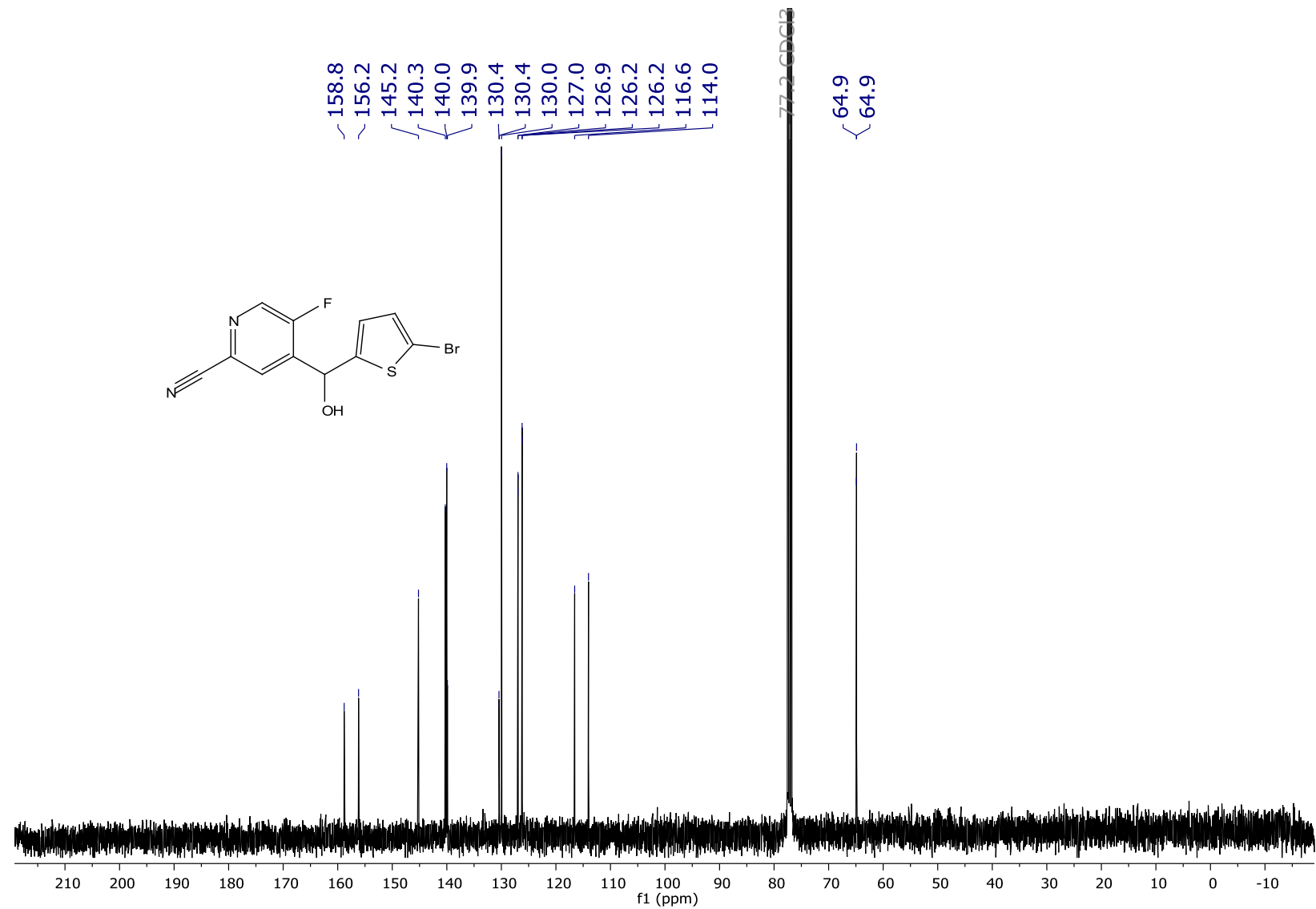

${ }^{13} \mathrm{C}\left\{{ }^{1} \mathrm{H}\right\}$ NMR $\left(101 \mathrm{MHz}, \mathrm{CDCl}_{3}\right)$ spectrum of $\mathbf{1 0 e}$. 


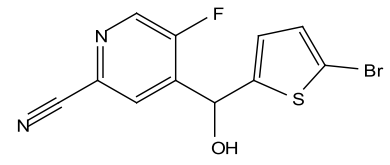

\begin{tabular}{llllllllllllllllllllllllllllllllllllllll}
\hline & 0 & -5 & -10 & -15 & -20 & -25 & -30 & -35 & -40 & -45 & -50 & -55 & -60 & -65 & -70 & -75 & -8 & -85 & -90 & -95 & -100 & -105 & -110 & -115 & -120 & -125 & -130 & -135 & -140 & -145 & -1
\end{tabular}

${ }^{19} \mathrm{~F}$ NMR (282 MHz, $\left.\mathrm{CDCl}_{3}\right)$ spectrum of $\mathbf{1 0 e}$.

ભ̊
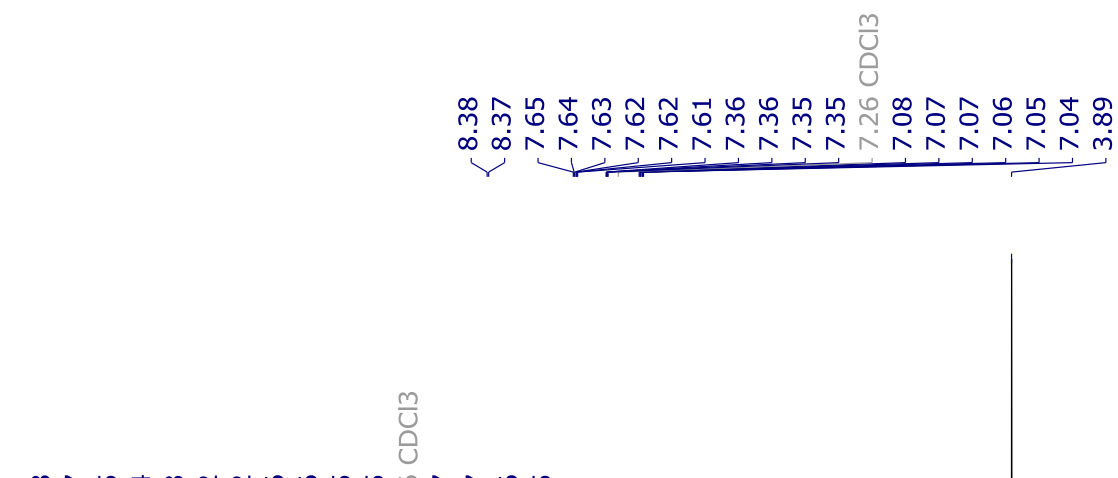

m

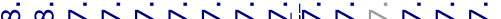
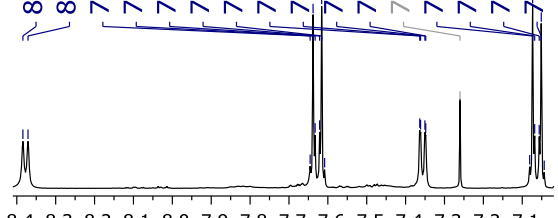

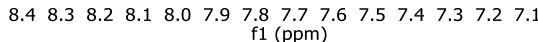
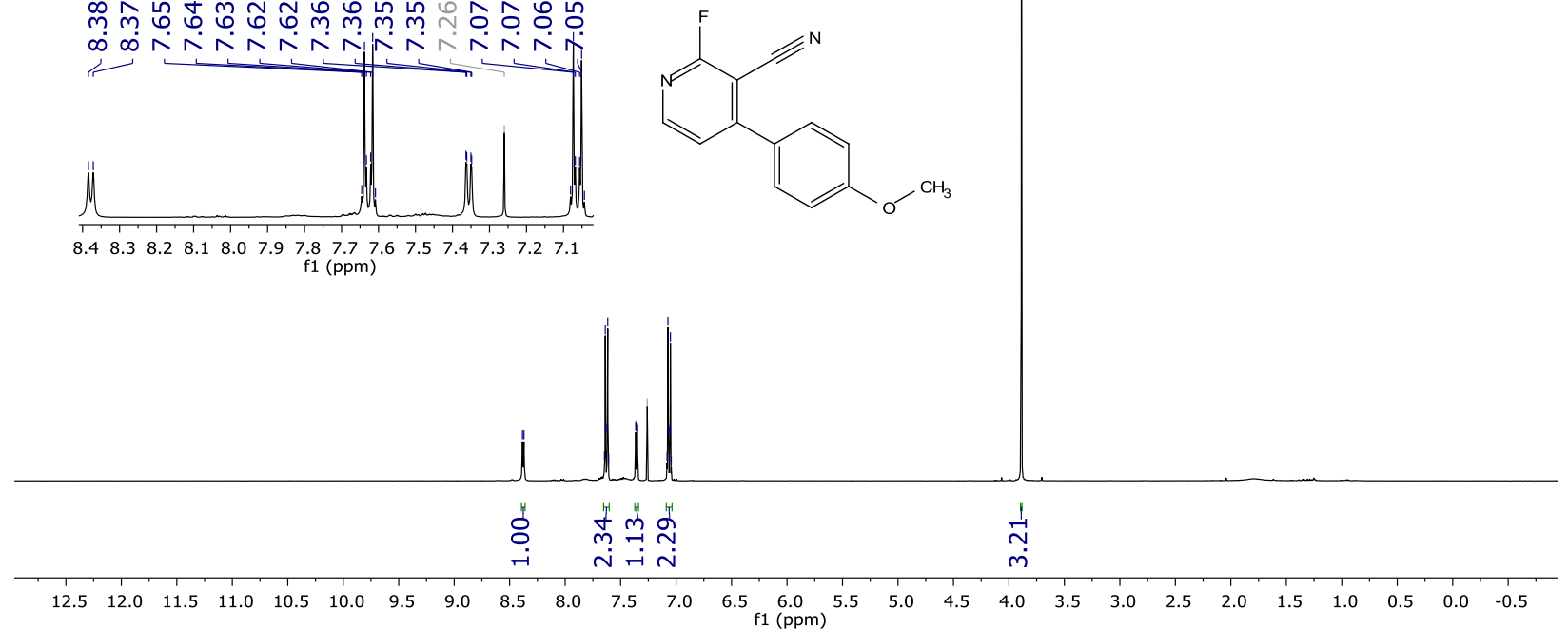

${ }^{1} \mathrm{H}$ NMR (400 MHz, $\mathrm{CDCl}_{3}$ ) spectrum of $\mathbf{1 0 f}$. 


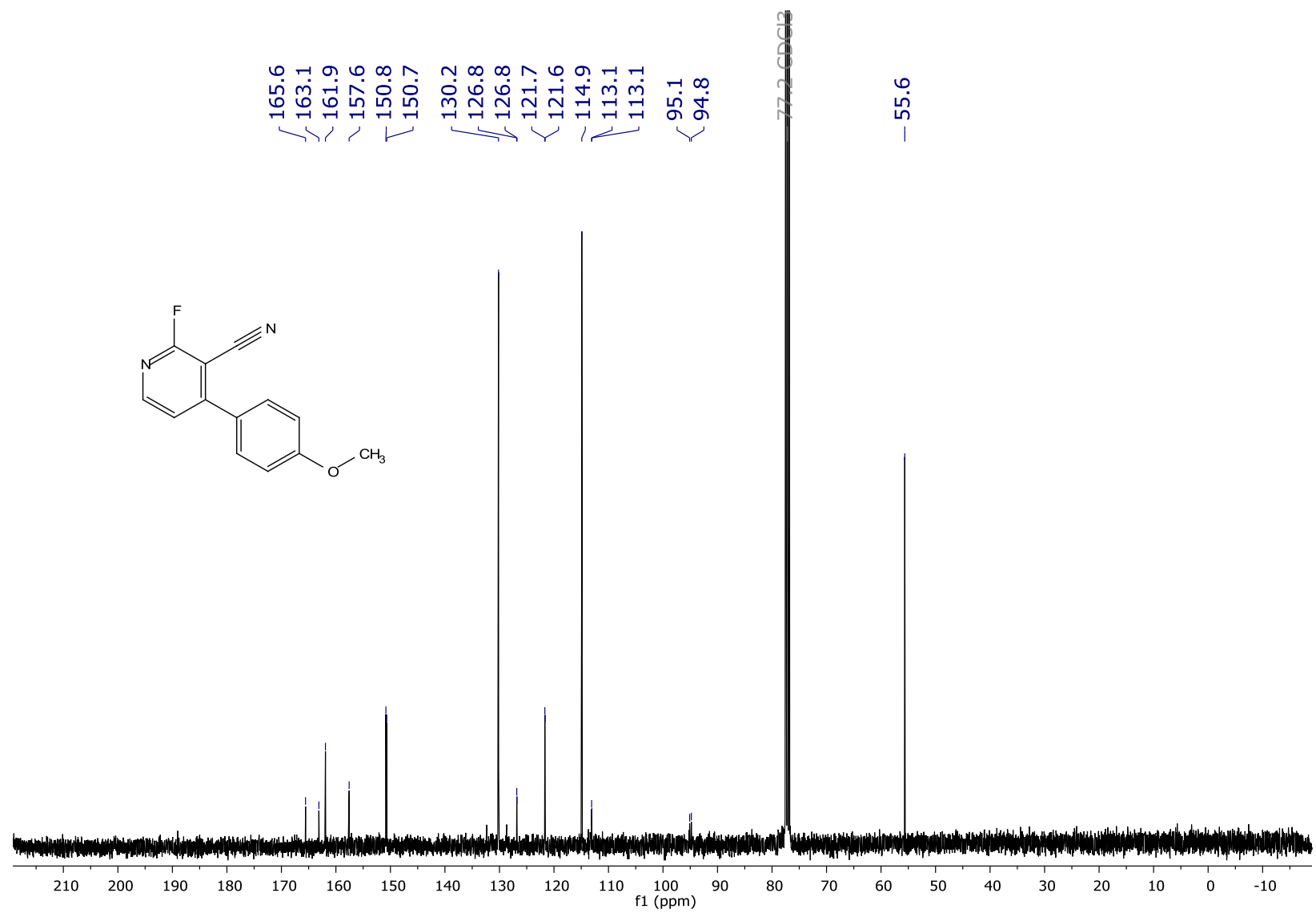

${ }^{13} \mathrm{C}\left\{{ }^{1} \mathrm{H}\right\} \mathrm{NMR}\left(101 \mathrm{MHz}, \mathrm{CDCl}_{3}\right)$ spectrum of $\mathbf{1 0 f}$.

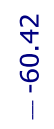
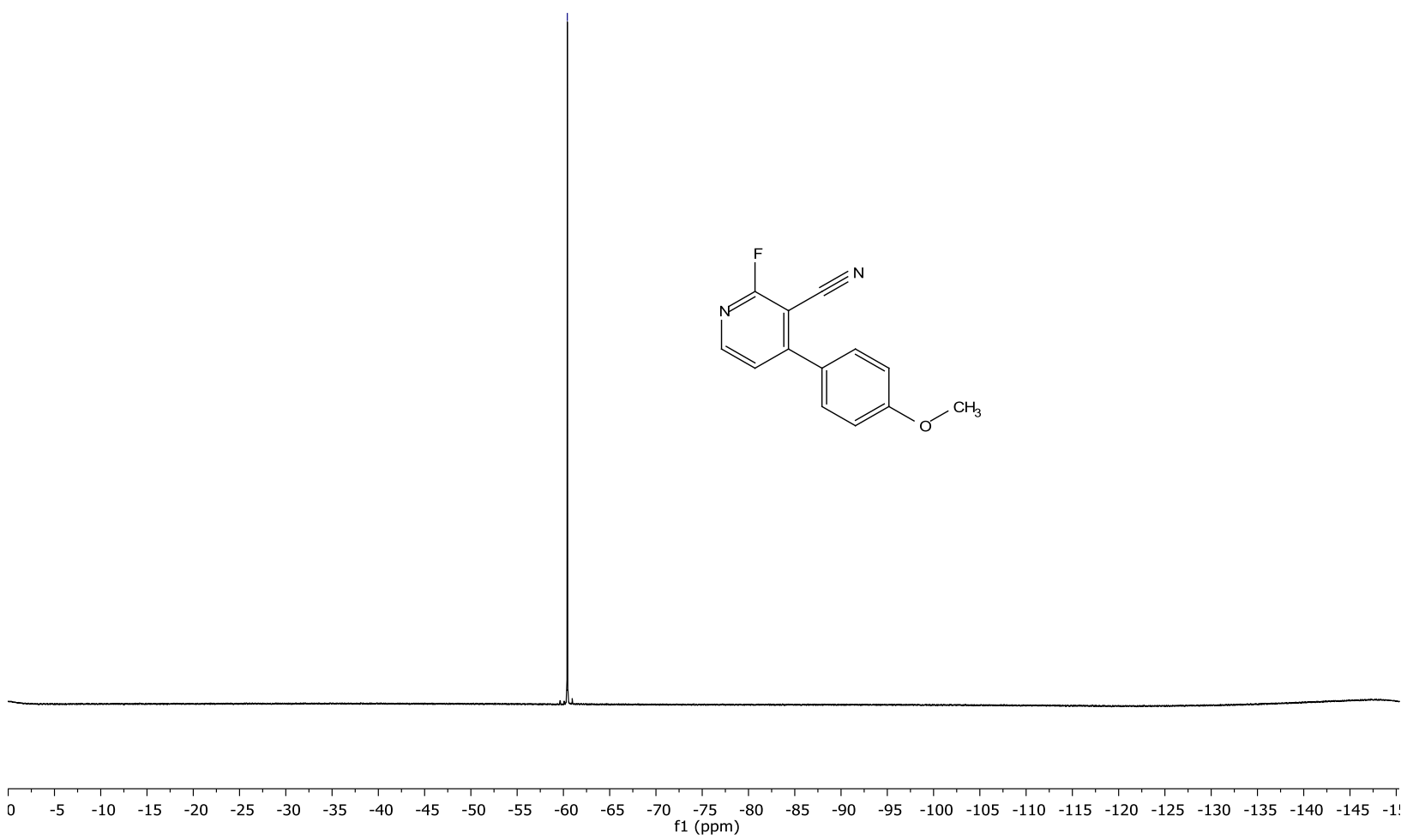

${ }^{19} \mathrm{~F}$ NMR (282 MHz, $\left.\mathrm{CDCl}_{3}\right)$ spectrum of $\mathbf{1 0 f}$.

S127 


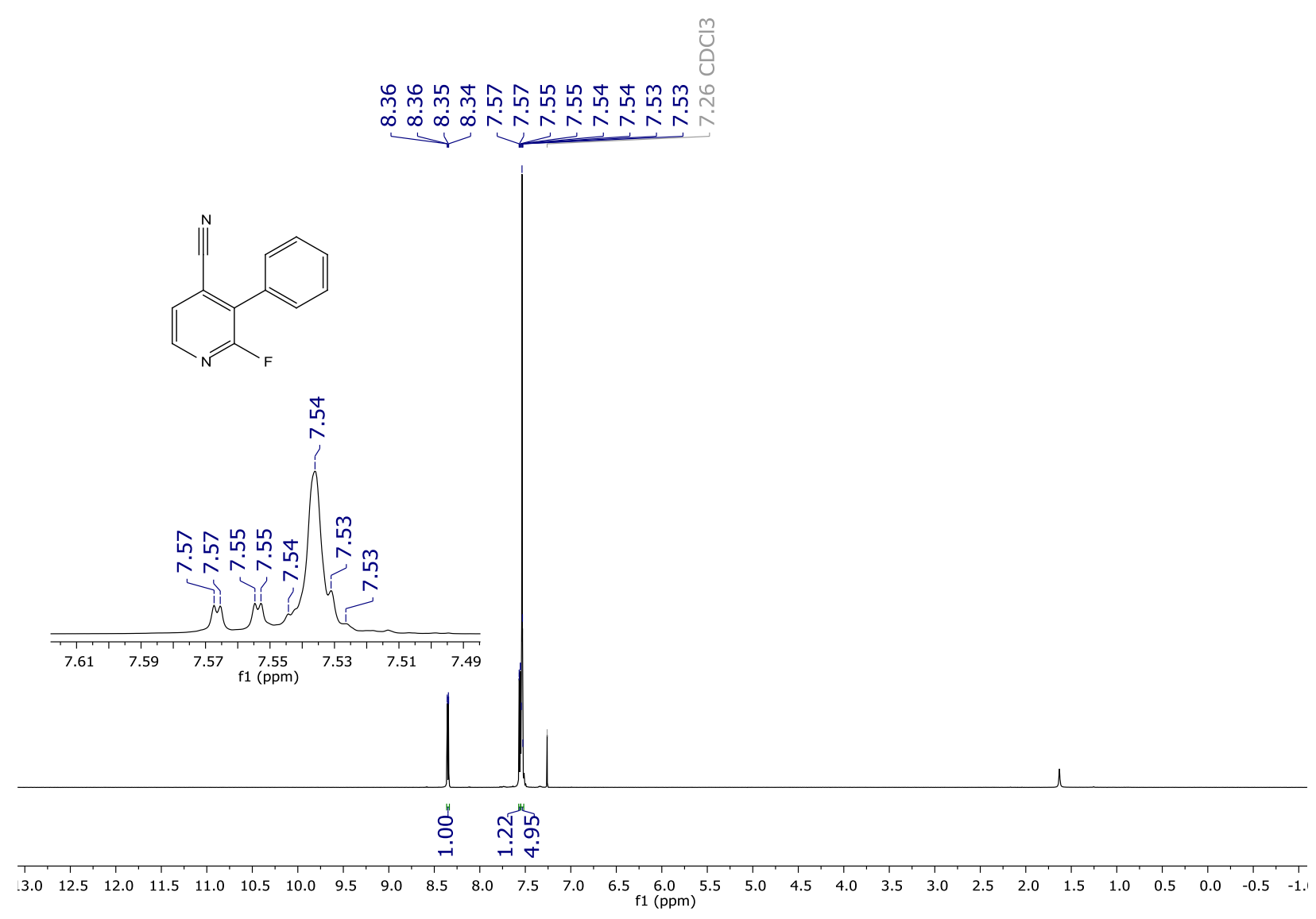

${ }^{1} \mathrm{H}$ NMR (400 MHz, $\mathrm{CDCl}_{3}$ ) spectrum of $\mathbf{1 0 g}$.

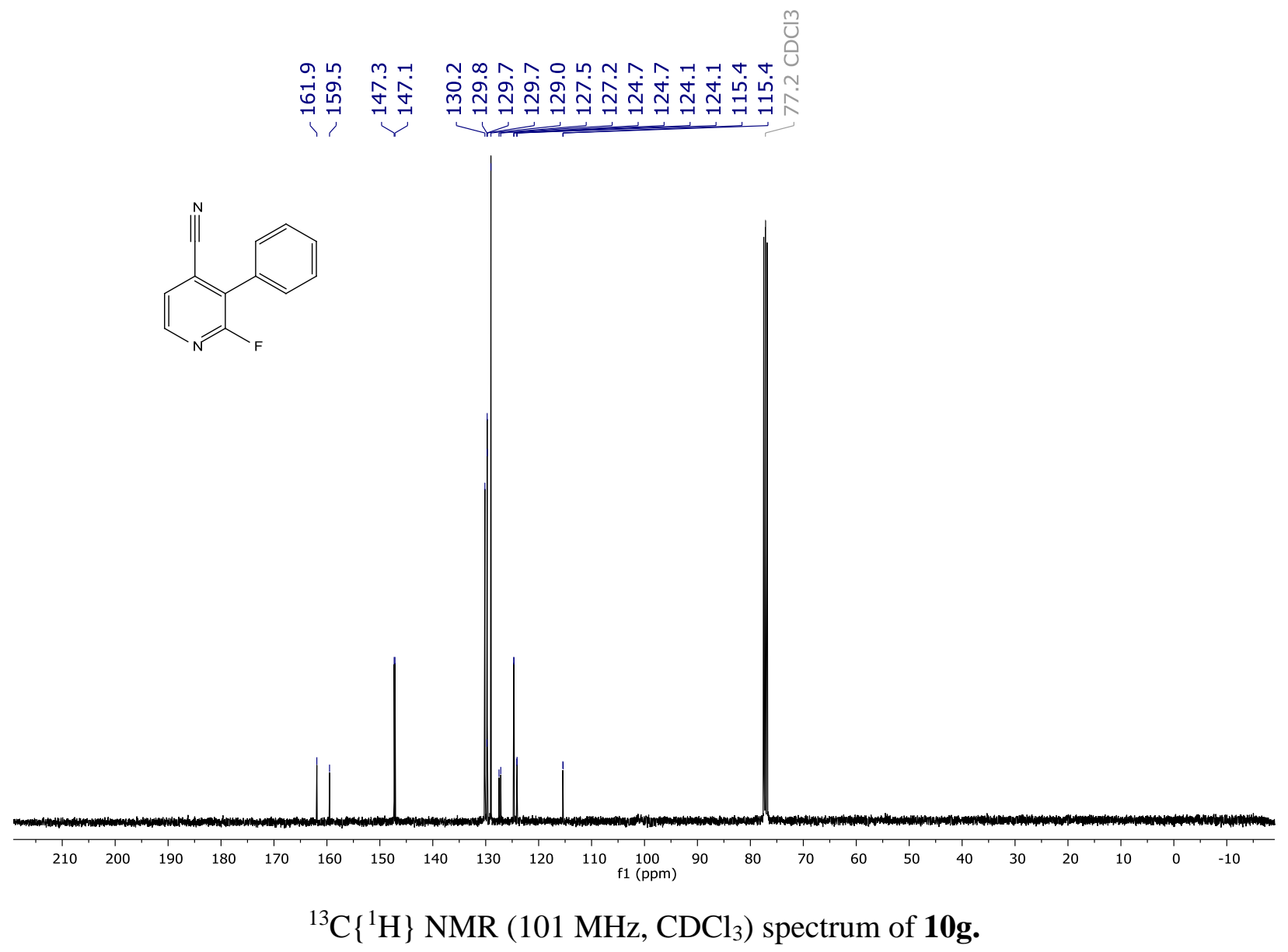



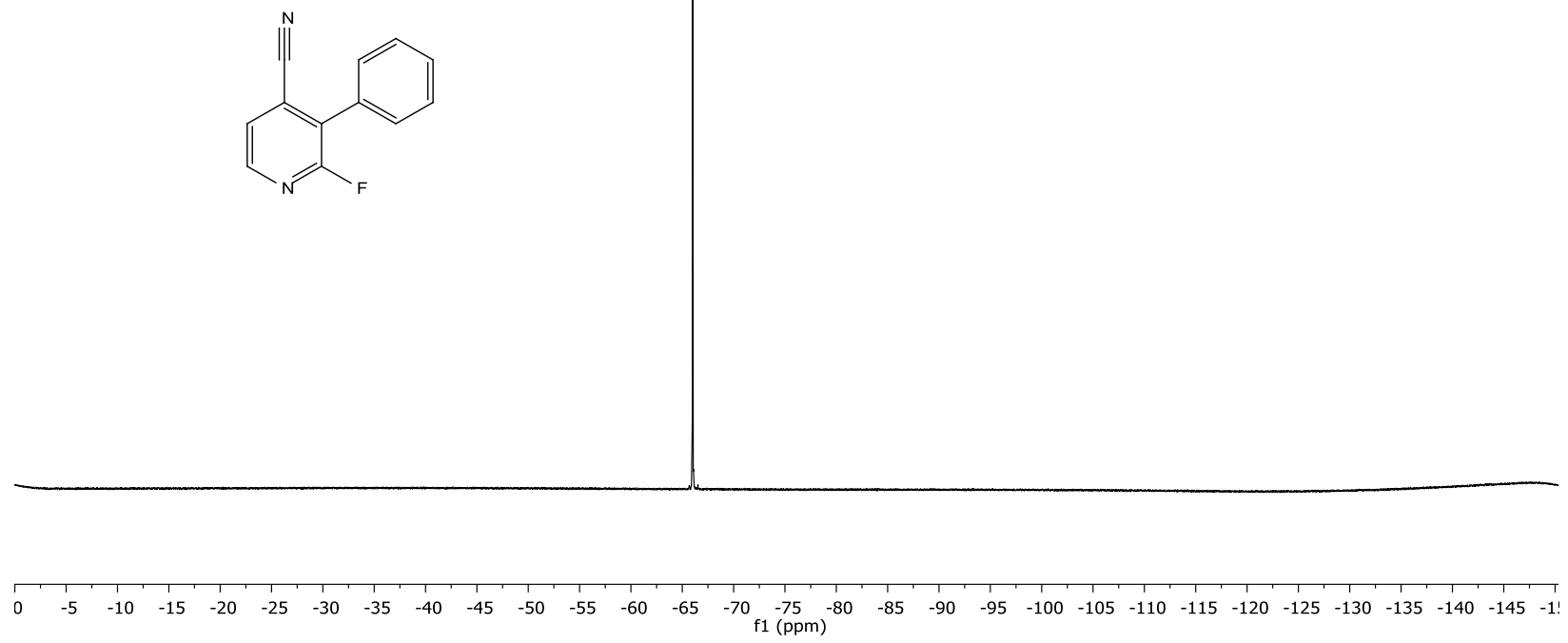

${ }^{19} \mathrm{~F}$ NMR (282 MHz, $\left.\mathrm{CDCl}_{3}\right)$ spectrum of $\mathbf{1 0 g}$.

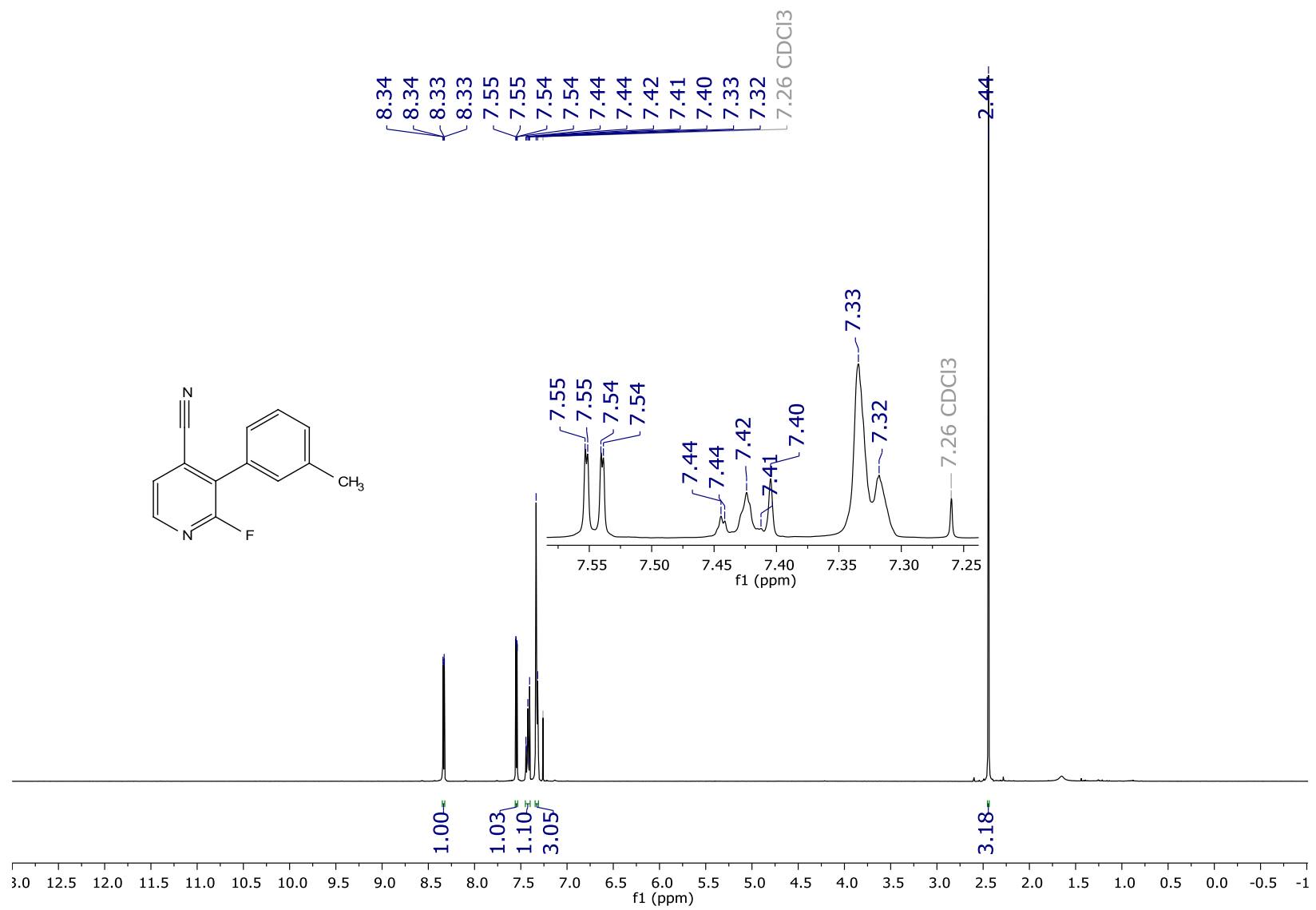

${ }^{1} \mathrm{H}$ NMR (400 MHz, $\mathrm{CDCl}_{3}$ ) spectrum of $\mathbf{1 0 h}$. 


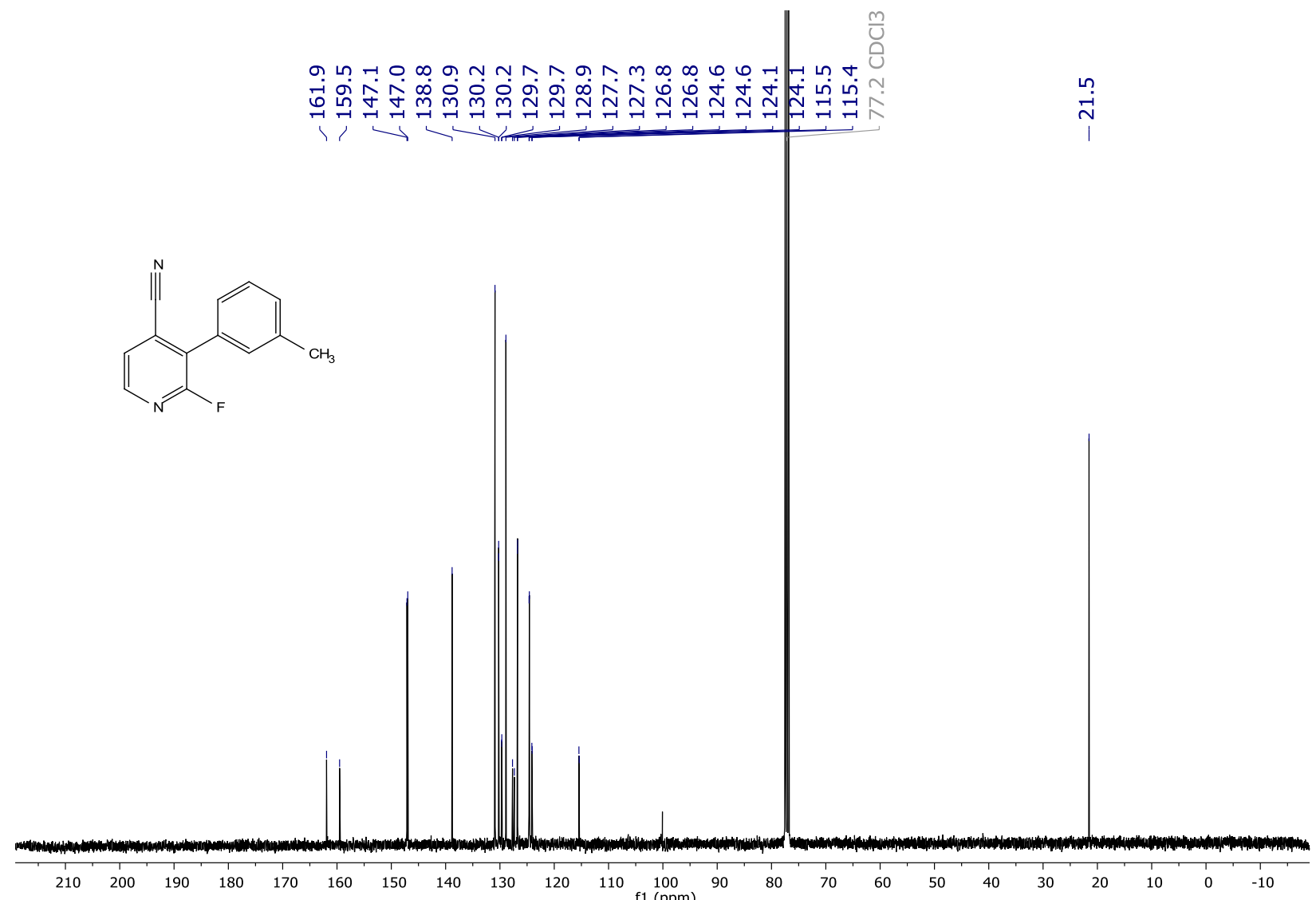

${ }^{13} \mathrm{C}\left\{{ }^{1} \mathrm{H}\right\}$ NMR $\left(101 \mathrm{MHz}, \mathrm{CDCl}_{3}\right)$ spectrum of $\mathbf{1 0 h}$.

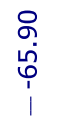

\begin{tabular}{llllllllllllllllllllllllllllllllllllllllllllllll}
\hline 0 & -5 & -10 & -15 & -20 & -25 & -30 & -35 & -40 & -45 & -50 & -55 & -60 & -65 & -70 & -75 & -80 & -85 & -90 & -95 & -100 & -105 & -110 & -115 & -120 & -125 & -130 & -135 & -140 & -145 & -1
\end{tabular}

${ }^{19} \mathrm{~F}$ NMR $\left(282 \mathrm{MHz}, \mathrm{CDCl}_{3}\right)$ spectrum of $\mathbf{1 0 h}$. 

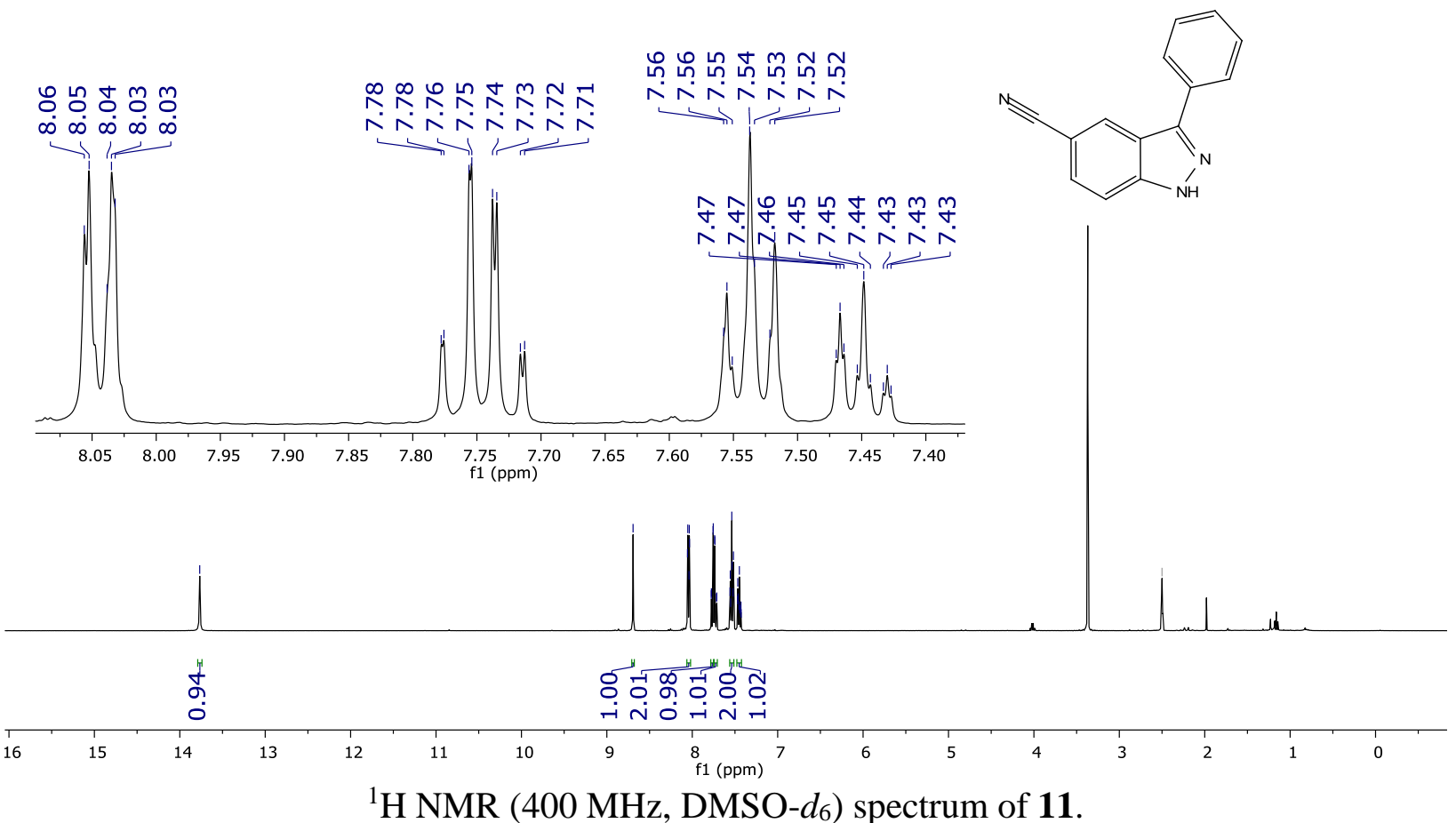

${ }^{1} \mathrm{H}$ NMR (400 MHz, DMSO-d6) spectrum of 11.

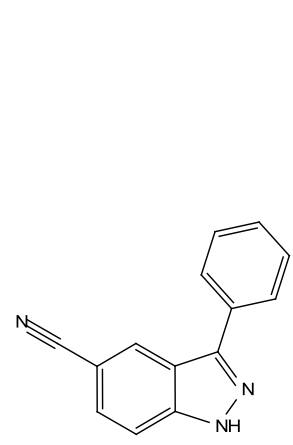

$0 \nabla+\pi \nabla m \infty r \infty \wedge N$ n

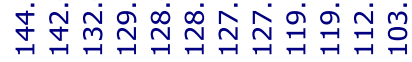

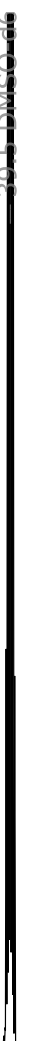


ஜ

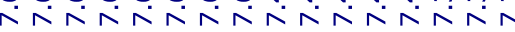
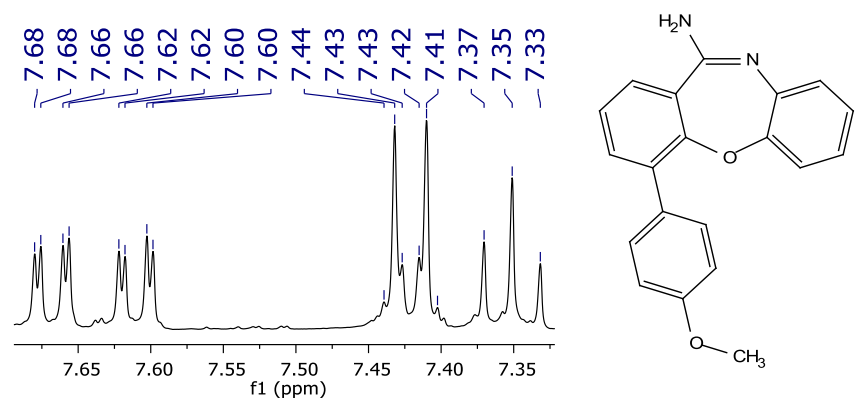

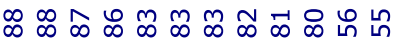

ف

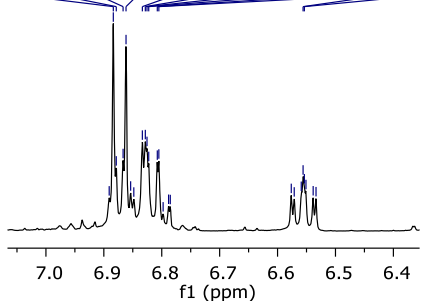

M

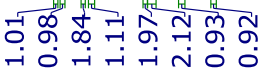

$\begin{array}{lllllllllllllll}13.0 & 12.5 & 12.0 & 11.5 & 11.0 & 10.5 & 10.0 & 9.5 & 9.0 & 8.5 & 8.0 & 7.5 & 7.0 & \left.\begin{array}{c}6.5 \\ \mathrm{f} 1(\mathrm{ppm})\end{array}\right)\end{array}$

${ }^{1} \mathrm{H}$ NMR $\left(400 \mathrm{MHz}, \mathrm{CDCl}_{3}\right)$ spectrum of $\mathbf{1 2}$.

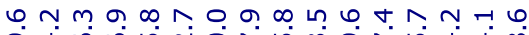
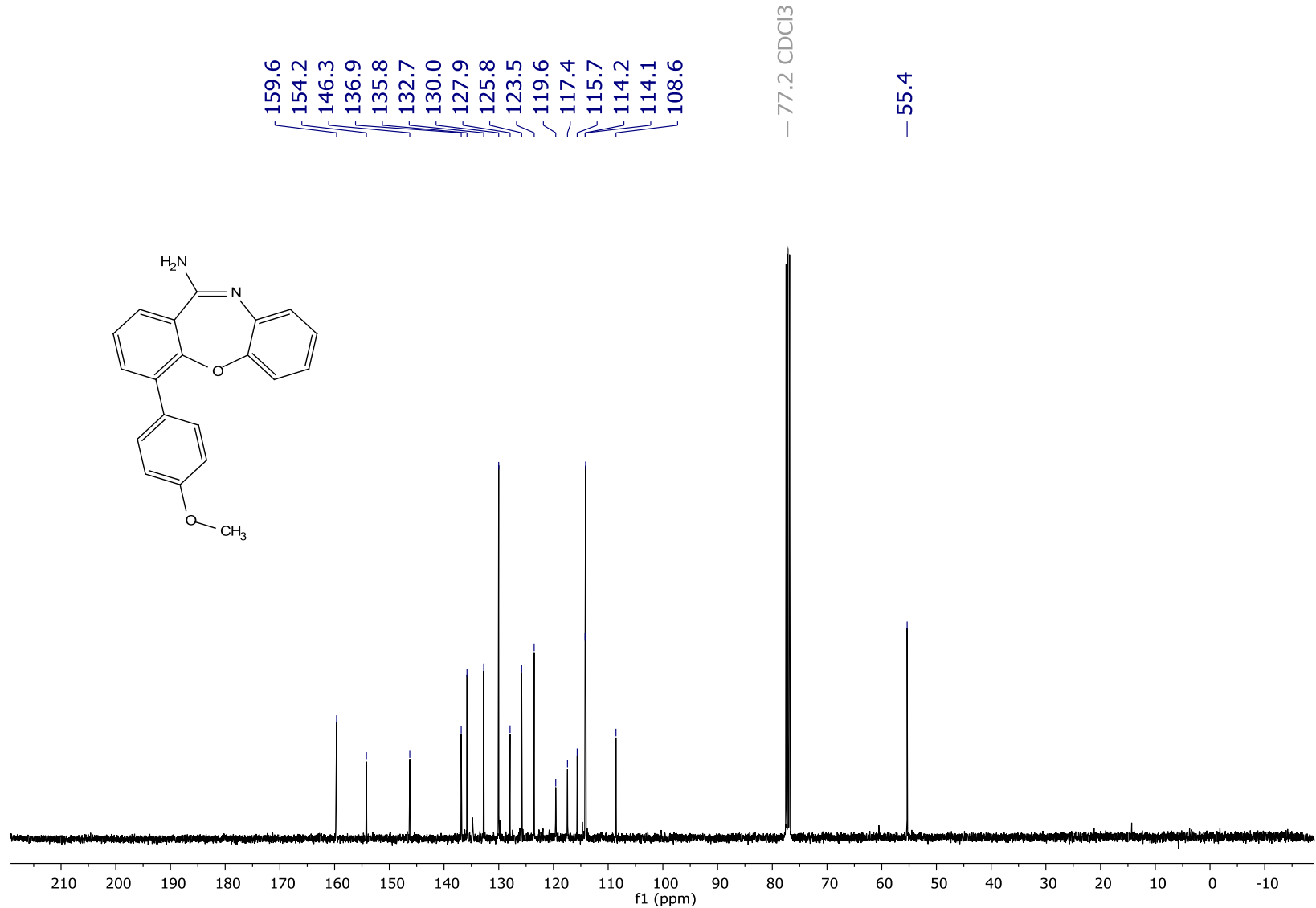

${ }^{13} \mathrm{C}\left\{{ }^{1} \mathrm{H}\right\}$ NMR $\left(101 \mathrm{MHz}, \mathrm{CDCl}_{3}\right)$ spectrum of $\mathbf{1 2}$. 